\title{
Insulin resistance in adolescents with overweight and obesity
}

Citation for published version (APA):

Dorenbos, E. (2020). Insulin resistance in adolescents with overweight and obesity: determinants and feasibility of lifestyle intervention. [Doctoral Thesis, Maastricht University]. ProefschriftMaken. https://doi.org/10.26481/dis.20200923ed

Document status and date:

Published: 01/01/2020

DOI:

10.26481/dis.20200923ed

Document Version:

Publisher's PDF, also known as Version of record

\section{Please check the document version of this publication:}

- A submitted manuscript is the version of the article upon submission and before peer-review. There can be important differences between the submitted version and the official published version of record.

People interested in the research are advised to contact the author for the final version of the publication, or visit the DOI to the publisher's website.

- The final author version and the galley proof are versions of the publication after peer review.

- The final published version features the final layout of the paper including the volume, issue and page numbers.

Link to publication

\footnotetext{
General rights rights.

- You may freely distribute the URL identifying the publication in the public portal. please follow below link for the End User Agreement:

www.umlib.nl/taverne-license

Take down policy

If you believe that this document breaches copyright please contact us at:

repository@maastrichtuniversity.nl

providing details and we will investigate your claim.
}

Copyright and moral rights for the publications made accessible in the public portal are retained by the authors and/or other copyright owners and it is a condition of accessing publications that users recognise and abide by the legal requirements associated with these

- Users may download and print one copy of any publication from the public portal for the purpose of private study or research.

- You may not further distribute the material or use it for any profit-making activity or commercial gain

If the publication is distributed under the terms of Article $25 \mathrm{fa}$ of the Dutch Copyright Act, indicated by the "Taverne" license above, 


\section{INSULIN RESISTANCE IN ADOLESCENTS WITH OVERWEIGHT AND OBESITY}

Determinants and feasibility of lifestyle intervention

Proefschrift

Elke Dorenbos 
(c) Elke Dorenbos, 2020, Maastricht

ISBN: 978-94-6380-832-3

Cover Design: Evelien Jagtman

Lay-out: RON Graphic Power \| www.ron.nu

Printed by: ProefschriftMaken || www.proefschriftmaken.nl

The research presented in this thesis was conducted within the Department of Paediatrics of Maastricht University Medical Centre, and NUTRIM School of Nutrition and Translational Research in Metabolism of Maastricht University. The studies were supported by the 7th Framework Programme of the European Commission. 


\section{INSULIN RESISTANCE IN ADOLESCENTS WITH OVERWEIGHT AND OBESITY}

Determinants and feasibility of lifestyle intervention

\section{Proefschrift}

ter verkrijging van de graad van doctor aan de Universiteit Maastricht,

op gezag van de Rector Magnificus, Prof dr. Rianne M. Letschert

volgens het besluit van het College van Decanen,

in het openbaar te verdedigen op

woensdag 23 september 2020 om 14.00 uur

door

Elke Dorenbos

Geboren te 20 juli 1989 te Steinheim, Duitsland 


\section{Promotor}

Prof. dr. M.S. Westerterp-Plantenga

\section{Copromotores}

Dr. A.C.E. Vreugdenhil

Dr. T.C. Adam

\section{Beoordelingscommissie}

Prof. dr. E. Dompeling, voorzitter

Prof. dr. E. Feskens, Wageningen University \& Research

Prof. dr. H Pijl, Leids Universitair Medisch Centrum

Prof. dr. R. Sverdlov

Prof. dr. E. van Mil 


\section{Table of contents}

Chapter 1 General introduction

Chapter 2 Sleep efficiency as a determinant of insulin sensitivity in overweight and obese adolescents

Chapter 3 PREVIEW: Prevention of diabetes through lifestyle intervention in a multicentre study in Europe in children (10-17y). Design, methods, and baseline results

Chapter 4 Effect of a high-protein low-Gl diet on insulin resistance in adolescents with overweight/obesity - a PREVIEW Randomized Controlled Trial

Chapter 5 Sleep duration is inversely related to BMI z-score in adolescents with overweight and obesity, independent of pubertal stage - a PREVIEW Study

Chapter 6 Role of aminotransferase concentration in insulin resistance and BMI z-score change in adolescents with overweight/obesity during intervention - a PREVIEW study

Chapter 7 General discussion

Appendices

Summary

Samenvatting

Valorisation

Dankwoord 157

About the author 161

List of publications 162 


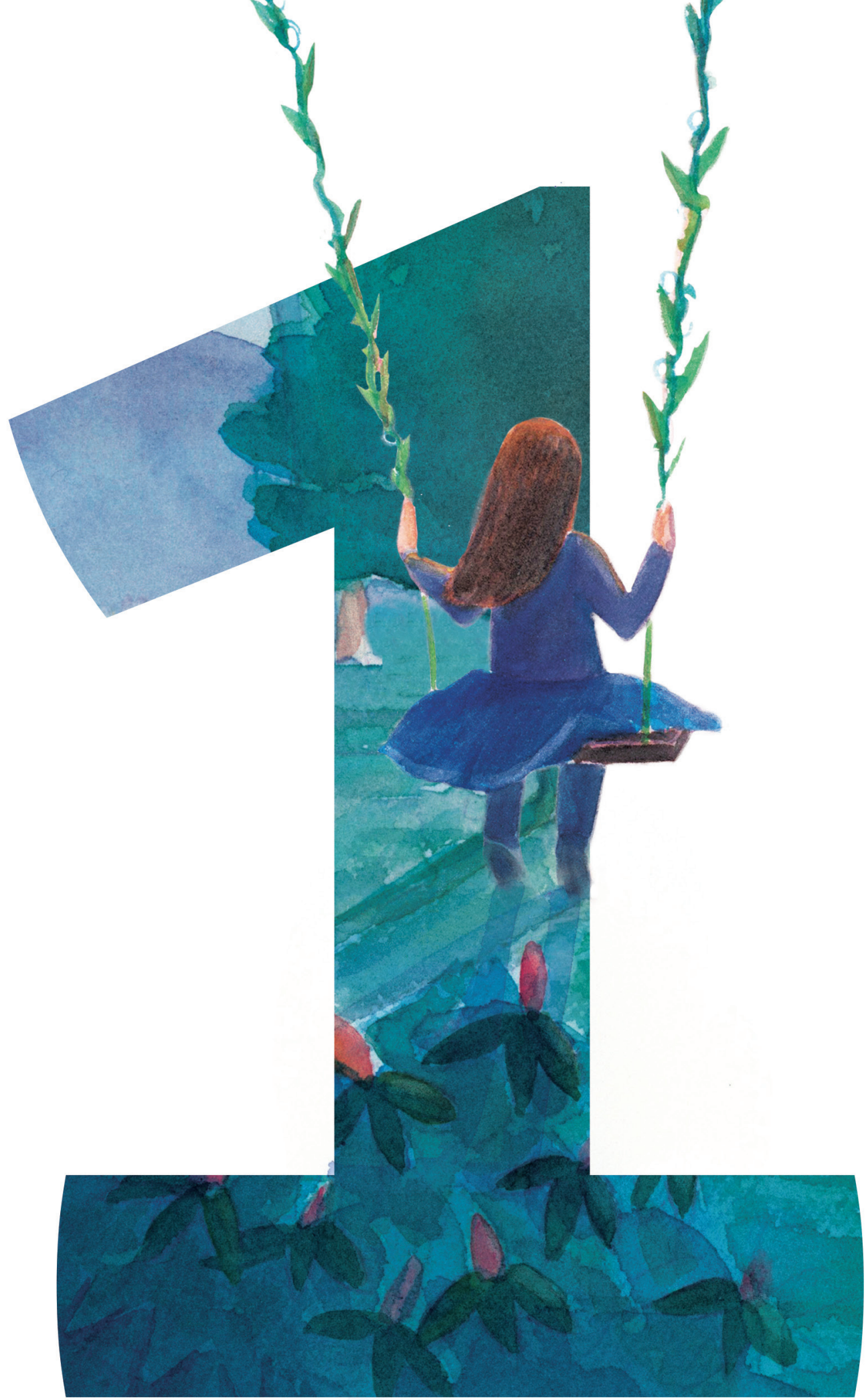




\section{Chapter 1}

\section{General introduction}


The WHO has identified obesity as one of the most important current global health burdens ${ }^{1}$. One of the major concerns of the obesity epidemic is the surge in obesity-related comorbidities in adolescents, such as insulin resistance (IR), dyslipidaemia and non-alcoholic fatty liver disease (NAFLD) ${ }^{2}$. Especially high insulin resistance has been related to increased risk of type 2 diabetes mellitus (T2DM) development early in life.

This thesis aims to provide an overview of the current knowledge of determinants related to pubertal insulin resistance in adolescents with overweight/obesity, as well as evaluate the effects and feasibility of a lifestyle intervention aiming to decrease IR in adolescents at risk.

\section{Childhood overweight and obesity}

Excess body fat is related to a wide range of comorbidities and health risks in children and adolescents ${ }^{3,4}$. Childhood obesity rarely develops as a consequence of underlying genetic, syndromic or endocrine conditions, but rather as a result of excess energy intake relative to energy expenditure over a long time ${ }^{5}$. Medical conditions resulting in overweight or obesity in childhood include hypothyroidism, growth hormone deficiency, Cushing's syndrome, Prader-Willi syndrome, Bardet-Biedl syndrome, defective leptin signalling and genetic factors, such as mutations in the melanocortin 4 receptor (MC4R) ${ }^{5,6}$. Also medications such as antipsychotics and thyreomimetics have been related to weight gain 7 . However, it is thought that only a few percent of all childhood obesity cases can be traced back to these secondary causes of obesity ${ }^{6}$. Most cases of childhood overweight and obesity are related to lifestyle-associated factors in our so-called "obesogenic" environment. For years, the amount of foods (in g) and beverages, caloric density of food, and energy intake per meal has increased both at home and while eating out, with a specific increase in snacks ${ }^{8,9}$. The increased availability and reduced costs of fast foods has resulted in increased intake of high-caloric snacks, sugar-sweetened beverages, high-glycaemic foods, fast foods containing high amounts of fat, and larger portion sizes ${ }^{6,7,70-12}$. Food intake is also associated with social class, where the intake of whole grains, lean meats and fresh vegetables and fruit were more commonly seen in participants with higher socio-economic status ${ }^{9}$. Decreased physical activity (PA) and increase in sedentary behaviour, which may be related to increased television and media use, are also contributors to the obesogenic environment ${ }^{13,14}$. Lastly, recently more evidence has emerged that early life factors such as maternal diabetes, breastfeeding and catch-up growth in early childhood may be related to increased risk of developing overweight and obesity at an early age $\mathrm{e}^{7,15}$.

Obesity in childhood is the most important predictor for overweight, obesity, and obesity-associated comorbidities in adulthood ${ }^{16-20}$. Even in childhood, a myriad of obesityrelated comorbidities has been described affecting nearly every organ system. Onset of puberty and further weight gain have been identified as the strongest predictors for 
transition of metabolically healthy obese to metabolically unhealthy obese status, and is related to increased morbidity and mortality ${ }^{19-21}$. One of such complications is glucose metabolism dysregulation, such as T2DM.

\section{Identifying adolescents at highest risk of T2DM development}

T2DM was typically considered to be an adult-onset disease that developed as a consequence of long-term glucose dysregulation and pancreatic $\beta$-cell failure ${ }^{22}$. With the childhood obesity epidemic, the incidence of T2DM in adolescents has increased and is now estimated to be $\sim 45 \%$ of all new diabetes cases in youth ${ }^{23}$. Especially worrying is that progression of impaired glucose tolerance to T2DM is much faster in adolescents compared to adults and can occur as fast as in 21 months $^{24}$. It is not yet feasible to study prevention of T2DM in adolescents as this would require extremely large cohorts and long follow-up time. Therefore diabetes prevention studies in children often use variables known to be precursors of, or associated with, T2DM to assess intervention effects. One of these is insulin resistance, which is the focus of this dissertation.

There is currently no uniformly accepted definition and cut-off value for IR in adolescents ${ }^{25}$. The gold standard technique for assessing IR is the hyperinsulinemic-euglycemic clamp, but due to it's invasive, expensive and time-consuming nature this is not achievable in paediatric practice ${ }^{26}$. Currently, approximately six techniques have been described for assessing IR in adolescents, of which the Homeostatic Model Assessment of Insulin Resistance (HOMA-IR) is the most commonly used due to it's inexpensive, easy and relatively non-invasive technique $27-29$. Cut-off values for HOMA-IR to define adolescents as insulin resistant ranged from 2.0-5.56 in adolescents of all age ${ }^{27}$ (Swindell \& Dorenbos, submitted). For detecting metabolic syndrome, cut-off points ranged between 1.7-2.6. A clearly defined method and cut-off value of assessing IR in adolescents will be beneficial in assessing normal and increased IR, prevalence of $I R$ in puberty and monitoring therapeutic outcomes. HOMA-IR between 0.5-1.0 is commonly observed in lean prepubertal adolescents, although adolescents with overweight/obesity frequently present with higher HOMA$I^{30,31}$. Therefore, in the studies in this thesis the HOMA-IR cut-off value of $>2.0$ was used for pubertal adolescents (Tanner stages $\geq 3$ ) or any HOMA-IR in prepubertal adolescents with overweight or obesity.

\section{Insulin resistance in adolescents with overweight and obesity}

IR is defined as a state of decreased sensitivity of tissues to insulin, which would induce hyperglycaemia, resulting in a compensatory increased insulin secretion by the pancreatic $\beta$-cell to maintain normal blood glucose concentrations ${ }^{32}$. IR is a major risk factor for the development of T2DM, and thought to play a key role in advancement of obesity-related comorbidities such as the metabolic syndrome $\mathrm{e}^{26,33,34}$. Depending on the method and cut-off value used, up to $44 \%$ of adolescents with overweight present with IR ${ }^{27}$. 
IR in adolescents is further complicated by a physiological increase in insulin resistance during puberty which is thought to facilitate growth ${ }^{35}$. Pubertal growth requires increased tissue proliferation, resulting in increased glucose demands by these tissues. The body's natural glucose reserves, known as glycogen stores, are small and the use of protein stores for producing new glucose will result in rapid muscle and protein loss ${ }^{35}$. In a state of IR the glucose uptake in certain tissues is decreased, leading to higher glucose availability for rapidly proliferating tissues.

IR in puberty normally follows a typical pattern, first described by Amiel et $a^{\beta 6}$. Typically, during puberty IR increases by $25-50 \%$, nadirs at mid-puberty (corresponding with Tanner stages 3-4) and then recovers when pubertal development is complete ${ }^{31,37,38}$. Although the exact mechanisms for pubertal IR are not fully understood yet, the increase in IR during puberty has been partly explained by sex, hormones and ethnicity. In multiple studies, girls were found to be more insulin resistant than boys, which might have been related to the increase in fat mass during puberty in girls ${ }^{27,37}$. One study found that sex differences disappeared when children had a $\mathrm{BMI}>27 \mathrm{~kg} / \mathrm{m} 2$, which might indicate that extreme adiposity might mask sex differences ${ }^{37}$.

Obesity has been identified as an important contributor to IR in adolescents. Crosssectional studies and one longitudinal study showed that adolescents with overweight and obesity had a similar but exaggerated pattern of IR during puberty, in which IR was higher at the onset of puberty, increased more during puberty, and did not always recover at the end of puberty ${ }^{31,32,37-42}$. IR appeared to increase with the level of obesity. Odds ratios for becoming insulin resistant were found to be 2.4 (1.2-4.9) and 6.0 (3.1-11.9) for boys and girls with overweight, and 9.1 (95\% Cl 4.0-20.4) and 13.2 (4.7-36.9) for boys and girls with obesity ${ }^{41}$. Mechanistically, excess fat mass and ectopic fat deposition are thought to be the main drivers for IR in adolescents, which is underlined by a study showing that increased visceral fat and fat depots in muscles and liver were directly related to the degree of IR ${ }^{43,44}$. Increased ectopic fat results in increased concentrations of free fatty acids (FFA), which decrease insulin sensitivity. FFA also alters mitochondrial function which produces increased reactive oxygen species (ROS), leading to intracellular endoplasmic reticulum dysfunction. This might result in defective insulin secretion of $\beta$-cells ${ }^{34}$. FFA and other adipocytokines released by adipose tissue induce a chronic inflammatory state, which also reduces muscle glucose uptake, furthering $\mathrm{IR}^{45}$. Familial factors, such as a family history of T2DM, have also been related to increased IR in adolescence although the importance of familial T2DM might differ between ethinicities ${ }^{33}$. Maternal gestational diabetes (GDM), small for gestational age (SGA) birth and catch-up growth early in life, too, have been related to $\mathrm{IR}^{46,47}$.

Progression from IR to more advanced glucose dysregulation stages, specifically impaired glucose tolerance (IGT) or impaired fasting glucose (IFG) and to T2DM in youth is preceded by $\beta$-cell dysfunction, which can be illustrated in a model by Cree-Green et al. (Figure 1.1) 32,33,48${ }^{51}$. In lean adolescents, rising insulin resistance during puberty is compensated by increased 

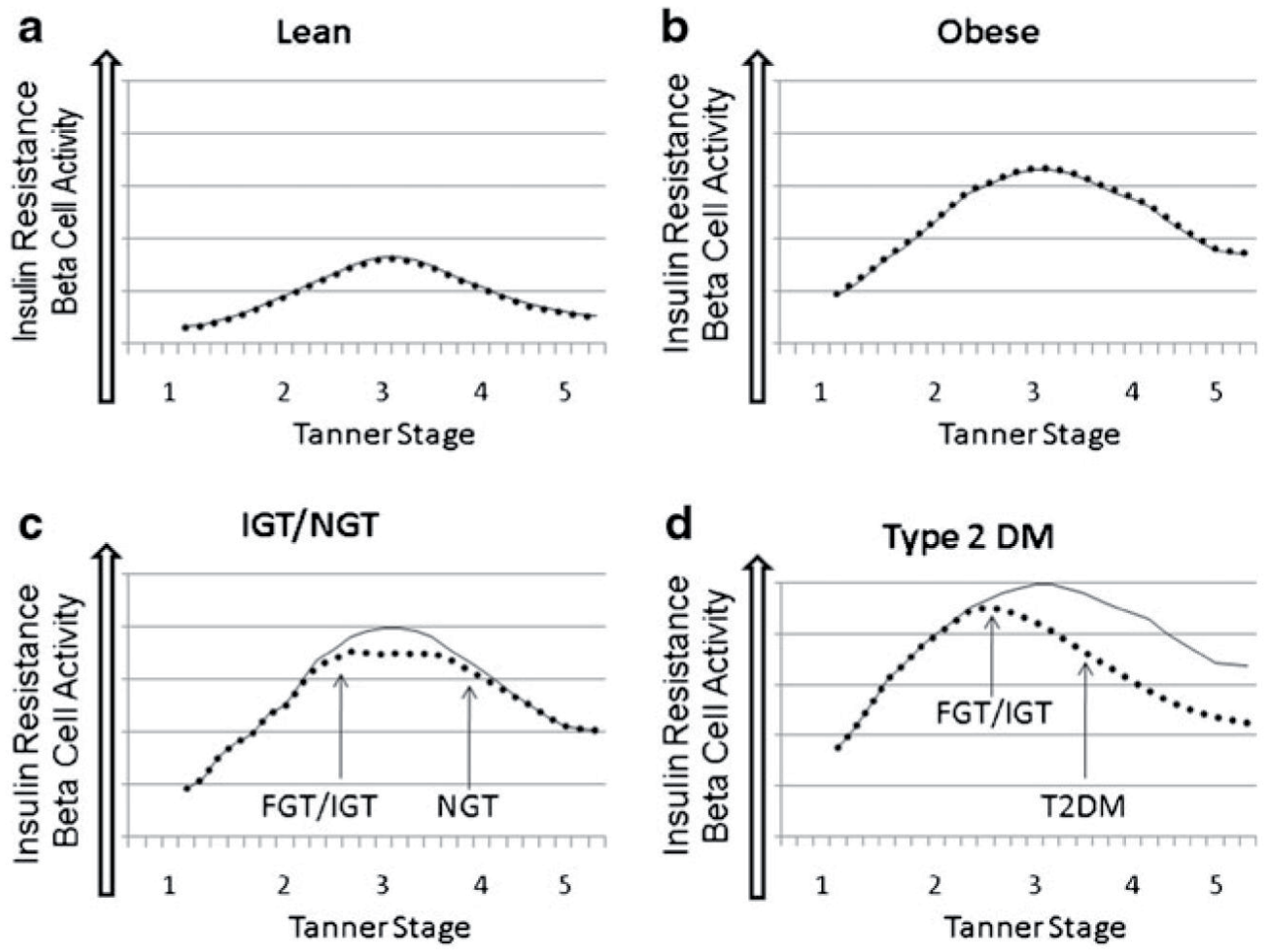

Figure 1.1: Patterns of insulin resistance and pancreatic $\beta$-cell function during puberty (as proposed by Cree-Green et $a^{\beta^{2}}$ ).

-- insulin resistance (IR), and ..... pancreatic $\beta$-cell function. In lean adolescents, during mid-puberty a mild increase in IR occurs, which is matched by a compensatory increased insulin production by the pancreatic $\beta$-cell so that normal glucose tolerance (NGT) remains (A). Adolescents with obesity show a more dramatic increase in IR and IR does not drop to pre-pubertal levels at the end of puberty. However, the increased IR is matched adequately by pancreatic insulin secretion and adolescents remain NGT (B). Adolescents with obesity and additional risk factors, such as unfavourable fat partitioning or a family history of T2DM, the increased IR and resulting increased insulin demands outreach pancreatic $\beta$-cell function and impaired glucose tolerance (IGT) occurs. As IR decreases towards the end of puberty, insulin secretion is sufficient again to match the IR and NGT occurs. These adolescents may be at risk for redevelopment of FGT/IGT again in the case of additional physiological stressors (Figure C). A small portion of adolescents with obesity, increased IR and FGT/IGT have continued insulin deficiency and will progress to develop type 2 diabetes mellitus (T2DM, Figure D).

insulin secretion from the pancreatic $\beta$-cell to maintain normoglycemia. In adolescents with additional risk factors, such as obesity, abnormal fat partitioning and a family history of T2DM, insulin demands outreach the insulin secreted by the pancreatic $\beta$-cell and FGT/ IGT may occur ${ }^{32}$. Hyperglycaemia, as a result of increased IR, is associated with low-grade inflammation which further impairs functioning of pancreatic $\beta$-cells ${ }^{32}$. Hyperglycaemia, increased insulin demands and stress are associated with $\beta$-cell hypertrophy and ultimately a rapid decline in number of $\beta$-cells ${ }^{32}$. Even if IR decreases towards the end of puberty, this pancreatic damage is permanent and hyperglycaemia may be persistent. The progression of FGT/IGT to T2DM in adolescents is faster than in adults and can occur in as little as 
21 months $^{24}$. Furthermore, IR and hyperglycaemia increase risk for the development of NAFLD, hypertriglycideamia, and cardiovascular dysfunction related to obesity ${ }^{42,52-54}$.

\section{Sleep is a possible third modifiable determinant for adolescent obesity and IR}

More evidence is emerging that sleep is a third modifiable contributor to energy balance, and consequently to overweight and obesity-related comorbidities. Short sleep and sleep debt have also been reported to be associated with increased risk of obesity in adults and children, and, albeit less strongly, IR in cross-sectional studies in children ${ }^{55-58}$. Even though there is still debate on what the optimal sleep time is for children and adolescents, sleep duration declines significantly during puberty ${ }^{59-62}$. An increasing number of studies linked sleep shortage and inadequate quality as independent risk factors for weight gain in youth, even after correction for BMI or screentime ${ }^{55,62,63}$. Moreover, sleep deprivation has been suggested to have a negative effect on markers of glucose metabolism. In a small but important study 21 adolescent boys were subjected to 3 nights of short sleep (4h/night) and 3 nights of long sleep (9h/night). HOMA-IR was significantly higher after the short sleep condition compared to after the long sleep condition ${ }^{64}$. The Cleveland Children's Sleep and Health Cohort observed an U-shape association between duration of sleep and HOMA-IR. Adolescents that slept 7.75 hours had the lowest HOMA-IR, but adolescents that slept either 5.0 or 10.5 hours had a $20 \%$ higher HOMA-IR.

It is likely that endocrine stress, as a consequence of too little or inadequate sleep quality, might drive these relationships with obesity and IR. Short sleep duration is related to increased cortisol concentrations, higher sympatic nerve system activation, high ghrelin concentrations and low leptin concentrations in the blood, all of which promote hunger and food consumption ${ }^{65,66}$. Sleep deprived individuals showed a preference for sugary and high caloric foods, and a decrease in physical activity ${ }^{56}$. However, most previous studies have been performed in a cross-sectional design and little is known yet about the effects of changes in sleep duration and architecture parameters on IR in adolescents with overweight and obesity. Until now, few studies have assessed the effects of sleep duration and sleep quality on obesity and IR in adolescents and children. Most studies however used a cross-sectional design or questionnaires as method of sleep assessment, but no objective measurements of sleep. Moreover, many studies did not correct for pubertal stage, sex or obesity status while all of these have been related to cardiometabolic risk factors ${ }^{58}$.

Identifying the effect of sleep on obesity status and the development of obesityrelated comorbidities in adolescents might aid in optimizing treatment strategies and prevent development of morbidities for adolescents with overweight and obesity. One PREVIEW substudy presented in this thesis is a proof-of-principle study that aimed to assess associations between sleep duration and architecture with intervention-related changes in BMI z-score and IR in adolescents with overweight and obesity. 


\section{NAFLD might affect BMI z-score and IR outcomes during intervention}

Non-alcoholic fatty liver disease is generally considered to be a consequence of obesity and IR, but very recent evidence has also suggested NAFLD to contribute to the development and maintenance of IR ${ }^{67,68}$. NAFLD encompasses a spectrum of liver diseases in the absence of excessive alcohol consumption ranging from simple hepatosteatosis ( $>5 \%$ fat) to liver cirrhosis, and has been related to obesity ${ }^{69,70}$. IR and NAFLD frequently co-develop in adolescents with overweight and obesity and appear to affect each other negatively. Especially circulating FFA were related to hyperinsulinemia, ectopic lipid deposition in muscles and the liver and a low-grade inflammatory state. The inflammation and elevated FFA concentrations reduced cellular glucose uptake, thereby inducing IR and a compensatory increase in pancreatic $\beta$-cell insulin secretion ${ }^{45}$. In a similar fashion in the liver excess fat accumulation and increased circulating FFA concentrations contributed to the development of NAFLD ${ }^{69-72}$. Thus, IR and NAFLD appear to reinforce one another, where IR is a driver for the development of NAFLD by contributing to hepatic steatosis, and NAFLD might exacerbate especially hepatic $I R^{67-73}$.

Interestingly, previous studies have demonstrated that adolescents who were insulin resistant were less successful in decreasing BMI z-score during intervention than adolescents that were not insulin resistant, suggesting that the presence of comorbidities might affect outcomes of lifestyle interventions targeting adolescent obesity ${ }^{50,74-77}$. It is not yet known how NAFLD relates to IR and BMI z-score outcomes during lifestyle intervention in adolescents with overweight/obesity. With up to $52 \%$ of adolescents with overweight presenting with IR and approximately one third with signs of NAFLD, it is relevant to assess not only the effect of interventions on BMI z-score and obesity-related comorbidities, but also whether the presence of IR and NAFLD relates to intervention-related outcomes in adolescents with overweight/obesity ${ }^{40,78}$.

Therefore a proof-of-principle substudy aiming to assess the prevalence and relationship between NAFLD and intervention-related outcomes on BMI z-score and HOMA-IR was conducted and included in this thesis.

\section{Treatment strategies for overweight, obesity and insulin resistance}

Taken together, increased IR during puberty, especially in adolescents with overweight and obesity, poses a risk for pancreatic $\beta$-cell exhaustion, T2DM development and a myriad of comorbidities. Pubertal IR might thus pose a risk for T2DM development, but puberty might also be a window of opportunity to prevent T2DM development by monitoring and preventing further IR increase. Considering the high prevalence of IR and obesity in adolescents, it is important to identify adolescents with IR in an early stage so that development of further morbidity may be prevented.

As of yet, no interventions in adolescents have been performed specifically aiming to decrease IR, although IR was often taken into account as a secondary intervention 
outcome in childhood obesity studies. International recommendations for the treatment of childhood overweight, obesity and obesity-related comorbidities advise comprehensive, multidisciplinary lifestyle interventions including dietary and PA strategies ${ }^{79,80}$. A metaanalysis suggested that macronutrient composition could be tailored to target specific cardiometabolic risk factors, such as $I^{81}$. Previous studies have shown that dietary strategies aimed at increasing protein intake resulted in a significant reduction in obesity and improvement of HOMA-IR ${ }^{74,82-88}$. The DioGenes study combined high protein intake with decreased glycaemic index (Gl) and observed a significant reduction in the percentage of adolescents with overweight/obesity in this group, compared to groups that followed different dietary strategies ${ }^{87}$. Other studies focussing on exercise strategies were shown to increase fat free mass and reduce fasting glucose concentration and HOMA-IR, with no exercise strategy resulting in more beneficial results than another ${ }^{89-92}$. Lifestyle interventions consisting of a combination of dietary, physical activity and behavioural strategies (such as counselling) were most effective in decreasing obesity status and cardiovascular risk parameters ${ }^{89}$. However, as of yet it is not known what the optimal combination is of dietary, exercise and behavioural strategies to decrease IR and BMI z-score in adolescents with overweight and obesity at high risk for T2DM development.

\section{The PREVIEW study in adolescents: researching the effects of a lifestyle strategy combining higher protein intake, lower Gl, and personalized PA recommendations for the prevention of diabetes in adolescents at risk}

The PREVention of diabetes through lifestyle Intervention and population studies in Europe and around the World (PREVIEW) study is a large, international randomized controlled trial in adults and adolescents that aimed to assess the effects of increasing dietary protein intake and reducing glycaemic index on T2DM prevention. In adolescents, the aim was to study the effects of a high-protein low-glycaemic index and moderate-protein moderateGI diet on IR in adolescents with overweight and obesity. Unique about the PREVIEW study in adolescents is that it was specifically targeted at adolescents with IR, as they were found to be at the highest risk for T2DM and possibly benefitted less from regular obesity interventions.

Adolescents were randomized into either a high-protein low-GI diet (HP, 25/45/30 En\% protein/carbohydrate/fat, GI >56) diet, or moderate-protein moderate-Gl diet (MP, 15/55/30 En\% protein/carbohydrates/fat, $\mathrm{Gl}<50$ ), and instructed to increase physical activity. During the two years study duration participants had frequent clinical investigation days where measurements were taken, compliance assessed and individuals received personalized dietary and PA counselling. It was hypothesized that a high-protein low-Gl diet would be superior in reducing IR increase during puberty, and decrease obesity status. 


\section{Outline of this dissertation}

In conclusion, exaggerated insulin resistance is an important consequence of childhood obesity and increases the risk of developing T2DM. Understanding more about the pattern, determinants and consequences of especially pathological IR in puberty might aid in developing therapeutic strategies to prevent future T2DM development. The aim of this thesis was to provide an overview of current risk factors related to pubertal IR, identify possible new determinants of pubertal IR, and to evaluate the feasibility and effects of a new lifestyle intervention on the prevention of T2DM development in adolescents at risk.

CHAPTER 2 presents an overview of risk factors for the development of pubertal insulin resistance, as well as associations of these risk factors with obesity status and IR in a population of adolescents with overweight or obesity.

In CHAPTER 3 the design of the PREVIEW lifestyle intervention in adolescents, which studies the effects of increasing protein intake and physical activity on insulin resistance in adolescents with overweight and obesity, is described. In addition, this chapter presents the baseline participant characteristics and associations with obesity status and IR. The results and feasibility of the PREVIEW study in adolescents are presented in CHAPTER 4.

CHAPTER 5 and 6 provide more insights in possible determinants of pubertal IR. CHAPTER $\mathbf{5}$ presents a proof-of-principle substudy in which objectively and subjectively measured sleep parameters were related to IR, BMI z-score, and intervention-mediated changes herein. In CHAPTER 6, the possible role of NAFLD on insulin resistance in adolescents with overweight and obesity is studied. The findings of this thesis and future perspectives for the research and treatment of insulin resistance in adolescents are discussed in CHAPTER 7. 


\section{REFERENCES}

1. WHO. Follow-up to the Political Declaration of the High-level Meeting of the General Assembly on the Prevention and Control of Non-Communicable Diseases. Global action plan for the prevention and control of NCDs 2013-2020. Geneva. 2013.

2. Lobstein T, Jackson-Leach R. Planning for the worst: estimates of obesity and comorbidities in school-age children in 2025. Pediatric obesity. 2016;11(5):321-325.

3. Zhu S, Wang Z, Shen W, Heymsfield SB, Heshka S. Percentage body fat ranges associated with metabolic syndrome risk: results based on the third National Health and Nutrition Examination Survey (1988-1994). The American Journal of Clinical Nutrition. 2003;78(2):228-235.

4. Freedman DS, Wang J, Maynard LM, et al. Relation of BMI to fat and fat-free mass among children and adolescents. International journal of obesity (2005). 2005;29(1):1-8.

5. Mason K, Page L, Balikcioglu PG. Screening for hormonal, monogenic, and syndromic disorders in obese infants and children. Pediatric annals. 2014;43(9):e218-224.

6. Kumar S, Kelly AS. Review of Childhood Obesity: From Epidemiology, Etiology, and Comorbidities to Clinical Assessment and Treatment. Mayo Clinic proceedings. 2017;92(2):251265.

7. Skelton JA, Irby MB, Grzywacz JG, Miller G. Etiologies of obesity in children: nature and nurture. Pediatric clinics of North America. 2011;58(6):1333-ix.

8. Kant AK, Graubard BI. Secular trends in patterns of self-reported food consumption of adult Americans: NHANES 1971-1975 to NHANES 1999-2002. The American journal of clinical nutrition. 2006;84(5):1215-1223.

9. Nielsen SJ, Popkin BM. Patterns and trends in food portion sizes, 1977-1998. Jama. 2003;289(4):450-453.

10. Piernas C, Popkin BM. Food portion patterns and trends among U.S. children and the relationship to total eating occasion size, 1977-2006. The Journal of nutrition. 2011;141(6):11591164.

11. de Ruyter JC, Olthof MR, Seidell JC, Katan MB. A trial of sugar-free or sugar-sweetened beverages and body weight in children. The New England journal of medicine. 2012;367(15):13971406.

12. McConahy KL, Smiciklas-Wright $\mathrm{H}$, Mitchell DC, Picciano MF. Portion size of common foods predicts energy intake among preschool-aged children. Journal of the American Dietetic Association. 2004;104(6):975-979.

13. Nelson MC, Neumark-Stzainer D, Hannan PJ, Sirard JR, Story M. Longitudinal and secular trends in physical activity and sedentary behavior during adolescence. Pediatrics. 2006;118(6):e16271634.

14. Cliff DP, Hesketh KD, Vella SA, et al. Objectively measured sedentary behaviour and health and development in children and adolescents: systematic review and meta-analysis. Obesity reviews : an official journal of the International Association for the Study of Obesity. 2016;17(4):330344.

15. Woo Baidal JA, Locks LM, Cheng ER, Blake-Lamb TL, Perkins ME, Taveras EM. Risk Factors for Childhood Obesity in the First 1,000 Days: A Systematic Review. American journal of preventive medicine. 2016;50(6):761-779.

16. Reilly JJ, Kelly J. Long-term impact of overweight and obesity in childhood and adolescence on morbidity and premature mortality in adulthood: systematic review. International journal of obesity (2005). 2011;35(7):891-898.

17. Singh AS, Mulder C, Twisk JW, van Mechelen W, Chinapaw MJ. Tracking of childhood overweight into adulthood: a systematic review of the literature. Obes Rev. 2008;9(5):474-488.

18. The NS, Suchindran C, North KE, Popkin BM, Gordon-Larsen P. The Association of Adolescent Obesity with Risk of Severe Obesity in Adulthood. JAMA : the journal of the American Medical Association. 2010;304(18):2042-2047. 
19. Franks PW, Hanson RL, Knowler WC, Sievers ML, Bennett PH, Looker HC. Childhood Obesity, Other Cardiovascular Risk Factors, and Premature Death. New England Journal of Medicine. 2010;362(6):485-493.

20. Li S, Chen W, Srinivasan SR, Xu J, Berenson GS. Relation of childhood obesity/cardiometabolic phenotypes to adult cardiometabolic profile: the Bogalusa Heart Study. American journal of epidemiology. 2012;176 Suppl 7(Suppl 7):S142-S149.

21. Reinehr T, Wolters B, Knop C, Lass N, Holl RW. Strong effect of pubertal status on metabolic health in obese children: a longitudinal study. J Clin Endocrinol Metab. 2015;100(1):301-308.

22. Ramlo-Halsted BA, Edelman SV. The natural history of type 2 diabetes. Implications for clinical practice. Primary care. 1999;26(4):771-789.

23. Goran MI, Ball GD, Cruz ML. Obesity and risk of type 2 diabetes and cardiovascular disease in children and adolescents. The Journal of clinical endocrinology and metabolism. 2003;88(4):14171427.

24. Kleber M, Lass N, Papcke S, Wabitsch M, Reinehr T. One-year follow-up of untreated obese white children and adolescents with impaired glucose tolerance: high conversion rate to normal glucose tolerance. Diabetic medicine : a journal of the British Diabetic Association. 2010;27(5):516521.

25. American Diabetes Association. Classification and Diagnosis of Diabetes. Diabetes care. 2016;39(Supplement 1):S13-S22.

26. Levy-Marchal C, Arslanian S, Cutfield W, et al. Insulin resistance in children: consensus, perspective, and future directions. The Journal of clinical endocrinology and metabolism. 2010;95(12):5189-5198.

27. van der Aa MP, Fazeli Farsani S, Knibbe CA, de Boer A, van der Vorst MM. Population-Based Studies on the Epidemiology of Insulin Resistance in Children. Journal of diabetes research. 2015;2015:362375.

28. Matthews DR, Hosker JP, Rudenski AS, Naylor BA, Treacher DF, Turner RC. Homeostasis model assessment: insulin resistance and beta-cell function from fasting plasma glucose and insulin concentrations in man. Diabetologia. 1985;28(7):412-419.

29. Conwell LS, Trost SG, Brown WJ, Batch JA. Indexes of insulin resistance and secretion in obese children and adolescents: a validation study. Diabetes care. 2004;27(2):314-319.

30. Jeffery AN, Metcalf BS, Hosking J, Streeter AJ, Voss LD, Wilkin TJ. Age before stage: insulin resistance rises before the onset of puberty: a 9-year longitudinal study (EarlyBird 26). Diabetes care. 2012;35(3):536-541.

31. Xu L, Li M, Yin J, et al. Change of Body Composition and Adipokines and Their Relationship with Insulin Resistance across Pubertal Development in Obese and Nonobese Chinese Children: The BCAMS Study. International journal of endocrinology. 2012;2012:389108.

32. Cree-Green M, Triolo TM, Nadeau KJ. Etiology of insulin resistance in youth with type 2 diabetes. Current diabetes reports. 2013;13(1):81-88.

33. Lee JM. Insulin resistance in children and adolescents. Reviews in endocrine \& metabolic disorders. 2006;7(3):141-147.

34. Kelsey MM, Zeitler PS. Insulin Resistance of Puberty. Current diabetes reports. 2016;16(7):64.

35. Soeters MR, Soeters PB. The evolutionary benefit of insulin resistance. Clinical nutrition (Edinburgh, Scotland). 2012;31(6):1002-1007.

36. Amiel SA, Sherwin RS, Simonson DC, Lauritano AA, Tamborlane WV. Impaired insulin action in puberty. A contributing factor to poor glycemic control in adolescents with diabetes. The New England journal of medicine. 1986;315(4):215-219.

37. Moran A, Jacobs DR, Jr., Steinberger J, et al. Insulin resistance during puberty: results from clamp studies in 357 children. Diabetes. 1999;48(10):2039-2044. 
38. Goran MI, Gower BA. Longitudinal study on pubertal insulin resistance. Diabetes. 2001; 50(11):2444-2450.

39. Aradillas-Garcia C, Rodriguez-Moran M, Garay-Sevilla ME, Malacara JM, Rascon-Pacheco RA, Guerrero-Romero F. Distribution of the homeostasis model assessment of insulin resistance in Mexican children and adolescents. European journal of endocrinology. 2012;166(2):301-306.

40. Lee JM, Okumura MJ, Davis MM, Herman WH, Gurney JG. Prevalence and determinants of insulin resistance among U.S. adolescents: a population-based study. Diabetes care. 2006;29(11):24272432.

41. Caserta CA, Pendino GM, Alicante S, et al. Body mass index, cardiovascular risk factors, and carotid intima-media thickness in a pediatric population in southern Italy. Journal of pediatric gastroenterology and nutrition. 2010;51(2):216-220.

42. Bao W, Srinivasan SR, Berenson GS. Persistent elevation of plasma insulin levels is associated with increased cardiovascular risk in children and young adults. The Bogalusa Heart Study. Circulation. 1996;93(1):54-59.

43. Bennett B, Larson-Meyer DE, Ravussin E, et al. Impaired insulin sensitivity and elevated ectopic fat in healthy obese vs. nonobese prepubertal children. Obesity (Silver Spring). 2012;20(2):371375.

44. Abreu GdA, Barufaldi LA, Bloch KV, Szklo M. A Systematic Review on Sleep Duration and Dyslipidemia in Adolescents: Understanding Inconsistencies. Arquivos Brasileiros de Cardiologia. 2015;105(4):418-425.

45. Cruz ML, Shaibi GQ, Weigensberg MJ, Spruijt-Metz D, Ball GD, Goran MI. Pediatric obesity and insulin resistance: chronic disease risk and implications for treatment and prevention beyond body weight modification. Annual review of nutrition. 2005;25:435-468.

46. Nicholas LM, Morrison JL, Rattanatray L, Zhang S, Ozanne SE, McMillen IC. The early origins of obesity and insulin resistance: timing, programming and mechanisms. International journal of obesity (2005). 2016;40(2):229-238.

47. Hofman PL, Regan F, Jackson WE, et al. Premature birth and later insulin resistance. The New England journal of medicine. 2004;351(21):2179-2186.

48. Gungor N, Hannon T, Libman I, Bacha F, Arslanian S. Type 2 diabetes mellitus in youth: the complete picture to date. Pediatr Clin North Am. 2005;52(6):1579-1609.

49. Cali AM, Man CD, Cobelli C, et al. Primary defects in beta-cell function further exacerbated by worsening of insulin resistance mark the development of impaired glucose tolerance in obese adolescents. Diabetes care. 2009;32(3):456-461.

50. Chiavaroli V, Giannini C, D'Adamo E, et al. Weight loss in obese prepubertal children: the influence of insulin resistance. Endocrine research. 2013;38(1):48-57.

51. Dubinina IA, Chistiakov DA, Eremina IA, et al. Studying progression from glucose intolerance to type 2 diabetes in obese children. Diabetes \& metabolic syndrome. 2014;8(3):133-137.

52. Khan UI, McGinn AP, Isasi CR, et al. Differences in Cardiometabolic Risk between InsulinSensitive and Insulin-Resistant Overweight and Obese Children. Childhood obesity (Print). 2015;11(3):289-296.

53. Asato Y, Katsuren K, Ohshiro T, Kikawa K, Shimabukuro T, Ohta T. Relationship between lipid abnormalities and insulin resistance in Japanese school children. Arteriosclerosis, thrombosis, and vascular biology. 2006;26(12):2781-2786.

54. Harris MI, Klein R, Welborn TA, Knuiman MW. Onset of NIDDM occurs at least 4-7 yr before clinical diagnosis. Diabetes care. 1992;15(7):815-819.

55. Cappuccio FP, Taggart FM, Kandala N-B, et al. Meta-Analysis of Short Sleep Duration and Obesity in Children and Adults. Sleep. 2008;31(5):619-626.

56. Garaulet M, Ortega FB, Ruiz JR, et al. Short sleep duration is associated with increased obesity markers in European adolescents: effect of physical activity and dietary habits. The HELENA study. International journal of obesity (2005). 2011;35(10):1308-1317. 
57. McClain JJ, Lewin DS, Laposky AD, Kahle L, Berrigan D. Associations between physical activity, sedentary time, sleep duration and daytime sleepiness in US adults. Preventive Medicine. 2014;66:68-73.

58. Quist JS, Sjödin A, Chaput J-P, Hjorth MF. Sleep and cardiometabolic risk in children and adolescents. Sleep Medicine Reviews. 2016;29:76-100.

59. Matricciani LA, Olds TS, Blunden S, Rigney G, Williams MT. Never Enough Sleep: A Brief History of Sleep Recommendations for Children. Pediatrics. 2012;129(3):548.

60. Knutson KL. The association between pubertal status and sleep duration and quality among a nationally representative sample of U. S. adolescents. American journal of human biology: the official journal of the Human Biology Council. 2005;17(4):418-424.

61. Thorleifsdottir B, Bjornsson JK, Benediktsdottir B, Gislason T, Kristbjarnarson H. Sleep and sleep habits from childhood to young adulthood over a 10-year period. Journal of psychosomatic research. 2002;53(1):529-537.

62. Rutters F, Gerver WJ, Nieuwenhuizen AG, Verhoef SPM, Westerterp-Plantenga MS. Sleep duration and body-weight development during puberty in a Dutch children cohort. International Journal Of Obesity. 2010;34:1508.

63. Fatima Y, Doi SAR, Mamun AA. Longitudinal impact of sleep on overweight and obesity in children and adolescents: a systematic review and bias-adjusted meta-analysis. Obesity Reviews. 2015;16(2):137-149.

64. Klingenberg L, Chaput JP, Holmback U, et al. Acute Sleep Restriction Reduces Insulin Sensitivity in Adolescent Boys. Sleep. 2013;36(7):1085-1090.

65. Hart CN, Carskadon MA, Considine RV, et al. Changes in Children's Sleep Duration on Food Intake, Weight, and Leptin. Pediatrics. 2013;132(6):e1473.

66. Schmid SM, Hallschmid M, Schultes B. The metabolic burden of sleep loss. The lancet Diabetes \& endocrinology. 2015;3(1):52-62.

67. Gruben N, Shiri-Sverdlov R, Koonen DP, Hofker MH. Nonalcoholic fatty liver disease: A main driver of insulin resistance or a dangerous liaison? Biochimica et biophysica acta. 2014;1842(11):23292343.

68. Valenti L, Bugianesi E, Pajvani U, Targher G. Nonalcoholic fatty liver disease: cause or consequence of type 2 diabetes? Liver international : official journal of the International Association for the Study of the Liver. 2016;36(11):1563-1579.

69. AlKhater SA. Paediatric non-alcoholic fatty liver disease: an overview. Obesity reviews : an official journal of the International Association for the Study of Obesity. 2015;16(5):393-405.

70. Bush H, Golabi P, Younossi ZM. Pediatric Non-Alcoholic Fatty Liver Disease. Children (Basel, Switzerland). 2017;4(6):48.

71. Jimenez-Rivera C, Hadjiyannakis S, Davila J, et al. Prevalence and risk factors for non-alcoholic fatty liver in children and youth with obesity. BMC Pediatrics. 2017;17(1):113.

72. Ozturk Y, Soylu OB. Fatty liver in childhood. World journal of hepatology. 2014;6(1):33-40.

73. Lonardo A, Ballestri S, Marchesini G, Angulo P, Loria P. Nonalcoholic fatty liver disease: a precursor of the metabolic syndrome. Digestive and liver disease : official journal of the Italian Society of Gastroenterology and the Italian Association for the Study of the Liver. 2015;47(3):181190.

74. Baxter KA, Ware RS, Batch JA, Truby H. Predicting success: factors associated with weight change in obese youth undertaking a weight management program. Obesity research \& clinical practice. 2013;7(2):e147-e154.

75. Cummings DM, Henes S, Kolasa KM, Olsson J, Collier D. Insulin resistance status: predicting weight response in overweight children. Archives of pediatrics \& adolescent medicine. 2008;162(8):764-768.

76. Maffeis C, Moghetti P, Grezzani A, Clementi M, Gaudino R, Tato L. Insulin resistance and the persistence of obesity from childhood into adulthood. The Journal of clinical endocrinology and metabolism. 2002;87(1):71-76. 
77. Travers $\mathrm{SH}$, Jeffers BW, Eckel RH. Insulin resistance during puberty and future fat accumulation. The Journal of clinical endocrinology and metabolism. 2002;87(8):3814-3818.

78. Yu EL, Golshan S, Harlow KE, et al. Prevalence of Nonalcoholic Fatty Liver Disease in Children with Obesity. The Journal of pediatrics. 2018.

79. Oude Luttikhuis $\mathrm{H}$, Baur L, Jansen $\mathrm{H}$, et al. Interventions for treating obesity in children. The Cochrane database of systematic reviews. 2009(1):Cd001872.

80. Force USPST. Screening for obesity in children and adolescents: Us preventive services task force recommendation statement. JAMA. 2017;317(23):2417-2426.

81. Gow ML, Ho M, Burrows $\mathrm{TL}$, et al. Impact of dietary macronutrient distribution on $\mathrm{BMI}$ and cardiometabolic outcomes in overweight and obese children and adolescents: a systematic review. Nutr Rev. 2014;72(7):453-470.

82. Damsgaard CT, Papadaki A, Jensen SM, et al. Higher protein diets consumed ad libitum improve cardiovascular risk markers in children of overweight parents from eight European countries. The Journal of nutrition. 2013;143(6):810-817.

83. Duckworth LC, Gately PJ, Radley D, Cooke CB, King RF, Hill AJ. RCT of a high-protein diet on hunger motivation and weight-loss in obese children: an extension and replication. Obesity (Silver Spring, Md). 2009;17(9):1808-1810.

84. Garnett SP, Gow M, Ho M, et al. Improved insulin sensitivity and body composition, irrespective of macronutrient intake, after a 12 month intervention in adolescents with pre-diabetes; RESIST a randomised control trial. BMC Pediatr. 2014;14:289.

85. Gately PJ, King NA, Greatwood HC, et al. Does a high-protein diet improve weight loss in overweight and obese children? Obesity (Silver Spring, Md). 2007;15(6):1527-1534.

86. Mirza NM, Palmer MG, Sinclair KB, et al. Effects of a low glycemic load or a low-fat dietary intervention on body weight in obese Hispanic American children and adolescents: a randomized controlled trial. The American journal of clinical nutrition. 2013;97(2):276-285.

87. Papadaki A, Linardakis M, Larsen TM, et al. The effect of protein and glycemic index on children's body composition: the DiOGenes randomized study. Pediatrics. 2010;126(5):e1143-1152.

88. Rolland-Cachera MF, Thibault $\mathrm{H}$, Souberbielle JC, et al. Massive obesity in adolescents: dietary interventions and behaviours associated with weight regain at 2 y follow-up. International journal of obesity and related metabolic disorders : journal of the International Association for the Study of Obesity. 2004;28(4):514-519.

89. Ho M, Garnett SP, Baur LA, et al. Impact of dietary and exercise interventions on weight change and metabolic outcomes in obese children and adolescents: a systematic review and metaanalysis of randomized trials. JAMA Pediatr. 2013;167(8):759-768.

90. Bell LM, Watts K, Siafarikas A, et al. Exercise alone reduces insulin resistance in obese children independently of changes in body composition. The Journal of clinical endocrinology and metabolism. 2007;92(11):4230-4235.

91. Shaibi GQ, Cruz ML, Ball GD, et al. Effects of resistance training on insulin sensitivity in overweight Latino adolescent males. Med Sci Sports Exerc. 2006;38(7):1208-1215.

92. Fedewa MV, Gist NH, Evans EM, Dishman RK. Exercise and insulin resistance in youth: a metaanalysis. Pediatrics. 2014;133(1):e163-174. 


\title{
Chapter 2
}

\section{Sleep efficiency as a determinant of insulin sensitivity in overweight and obese adolescents}

\author{
Elke Dorenbos \\ Jesse Rijks \\ Tanja Adam \\ Margriet Westerterp-Plantenga \\ Anita Vreugdenhil
}




\begin{abstract}
Insulin resistance (IR) occurs in a transient manner during puberty. Obese adolescents may be at risk for persistent insulin resistance during puberty. The objective of the study is to review the literature on the association of anthropometric and lifestyle characteristics with insulin sensitivity in overweight and obese adolescents, and include data from a new study. Relevant papers were selected and reviewed. In addition 137 overweight and obese adolescents (42m/95f, age 14.4 $\pm 2.3 y$, BMI z-score +3.3 \pm 0.7 , HOMA-IR 3.4 \pm 1.8 ) from the Centre for Overweight Adolescent and Children's Healthcare (MUMC+) were included in this study. Anthropometrics, Tanner stages, sleep characteristics, food intake behaviour, and physical activity were determined, and possible associations with homeostatic model assessment of insulin resistance (HOMA-IR) were tested. Adolescents with overweight and obesity with unfavourable fat partitioning and family history of non insulin-dependent diabetes mellitus (NIDDM) are at risk for persistent IR. Overweight and obese adolescents from the new cohort showed a higher HOMA-IR postpubertally. BMI z-score, age, pubertal stage and prepubertally total sleeping time (TST) and sleep efficiency (SE) were identified as significant contributors. Adolescents with overweight and obesity showed a persistently higher instead of transiently higher HOMA-IR during puberty, associated with BMI z-score, age, pubertal stage and prepubertally less TST and SE.
\end{abstract}




\section{INTRODUCTION}

During puberty, adolescents undergo a series of biological, cognitive and psychosocial changes. One of the hallmarks of puberty is a change in endocrine conditions and changes in anthropometric factors, such as height, body-weight, and body-composition. In parallel, behavioural changes, such as sleeping time, food intake behaviour and physical activity, driven both by biological processes or social and academic pressures occur ${ }^{1-9}$. Together, these factors pose an increased risk for the development of overweight and obesity, as well as for comorbidities such as insulin resistance, type 2 diabetes, cardiovascular disease, nonalcoholic fatty liver disease and obstructive sleep apnoea syndrome?

This review discusses insulin insensitivity during puberty, reviewing the existing evidence for possibly related changes in body-weight, especially overweight and obesity, sleep characteristics, physical activity and food intake behaviour. In addition, data from a new, recent study on relationships between sleep-efficiency and insulin sensitivity in overweight and obese adolescents are reported.

\section{Transient insulin resistance at puberty}

Puberty is associated with transient insulin resistance (IR) and hyperglycaemia, due to an impaired ability of insulin to stimulate glucose uptake ${ }^{10,11}$. In response, $\beta$-cells in the pancreas increase the production of insulin, leading to the clinical hallmark of high insulin concentrations in the presence of normal to high glucose concentrations. Insulin resistance, followed by impairment of insulin secretion can eventually lead to non-insulin dependent diabetes mellitus (NIDDM) ${ }^{10,11}$.

Insulin resistance is a transient physiological stage of normal development. Healthy children with normal weight experience a stage of physiological insulin resistance that starts to rise some years before puberty, peaking at mid-puberty and resolving to near prepubertal levels by the end of puberty (Tanner stage 5$)^{11-13}$. Girls however are more insulin resistant than boys at all Tanner stages ${ }^{11-13}$. In normal weight children the insulin resistance during puberty development is compensated by a sufficient pancreatic $\beta$-cell response to maintain glucose homeostasis $\mathrm{s}^{12-14}$. Other metabolic characteristics of pubertal insensitivity to insulin are decreased glucose oxidation and increased free fatty acid oxidation.

It is well known that insulin resistance is strongly associated with BMI and bodycomposition. Given that insulin resistance can occur during puberty in the absence of changes in BMI, and independent of changes in body fat, factors other than anthropometric changes have to be carefully examined in the onset of pubertal resistance ${ }^{11-13}$.

Due to the considerable change in endocrinological parameters, hormones may play a crucial role in the association of puberty and transient insulin resistance. One of the most essential changes accompanying pubertal development is the rise in sex hormone concentrations, from barely detectable levels in early childhood towards adult levels in late 
puberty. Insulin resistance, however, has not been shown to be associated with testosterone or estradiol levels. It has been hypothesized that the insulin resistance of normal puberty might be related to the GH/IGF-I axis. Plasma IGF-I levels are primarily regulated by $\mathrm{GH}$, and $\mathrm{GH}$ is a counterregulatory hormone known to be a potent insulin antagonist. Indeed, strong evidence was found for the contribution of hormonal changes in growth hormone/ IGF-1 to pubertal insulin resistance. Moran et al. demonstrated that IGF-I levels in boys and girls rise and fall during the course of puberty in concert with the well-recognized rise and fall in insulin resistance ${ }^{7}$. Insulin-like growth factor-binding protein 3 (IGFBP-3), the primary carrier protein of IGF-I, correlates positively with insulin resistance, whereas IGFBP-1, which is inhibited by insulin, correlates inversely with insulin resistance ${ }^{7}$. The data suggest that physiological elevation of the IGF-I/GH axis contributes to the relative insulin resistance of normal puberty.

Although pubertal insulin resistance is a normal physiological process compensated by a sufficient pancreatic $\beta$-cell response to maintain glucose homeostasis, it may contribute to pathology in the presence of pancreatic $\beta$-cell dysfunction in genetically/epigenetically at risk and obese children. Hyperglycaemia develops once the $\beta$-cell insulin secretion can no longer compensate for the level of IR. In children, the rate of passage from impaired glucose tolerance/impaired fasting glucose (IGT/IFG) to NIDDM happens faster compared to adults and can occur over the span of only 12-21 months. The majority of NIDDM in childhood concerns girls, consistent with their greater adiposity and IR.

IGT is also increasingly common in adolescents. However as they complete puberty, insulin sensitivity often is restored and hyperglycaemia resolves as the pancreas is able to meet insulin demands again. Cree-green et al. suggest that it may be that the transient IGT seen in these teens is similar to that of gestational diabetes mellitus (GDM), implying that these teens will be at increased risk of NIDDM later in life, compared with obese teens with no IGT ${ }^{14}$.

The divergence in the development of transient or persistent insulin resistance during puberty emphasizes the importance of longitudinal research. Goran et al. performed a longitudinal study in 60 children and examined at Tanner stage 1 and after 2.0 00.6 years of follow-up, by which time 29 children remained at Tanner stage 1 and 31 had progressed to Tanner stage 3 or $4^{15}$. They report that in children progressing to Tanner stage 3 , insulin sensitivity fell significantly by $32 \%$, acute insulin response increased by $30 \%$, the disposition index determined by an intravenous glucose tolerance test fell by $27 \%$, with a significant increase in fasting glucose and insulin. In children remaining at Tanner stage 1 however, there was a slight increase in insulin sensitivity, with no significant change in acute insulin response or fasting glucose and insulin. The pubertal fall in insulin resistance was more consistent in African-Americans, remained significant after controlling for age, sex, and change in fat mass, visceral fat, and fat- free mass, and was similar in children with low, medium, and high body fat. The pubertal transition from Tanner stage 1 to Tanner stage 3 
was associated with a $32 \%$ reduction in insulin sensitivity and increases in fasting glucose, insulin, and acute insulin response were similar across sex, ethnicity, and obesity. The significant fall in deposition index suggests conservation in $\beta$-cell function or an inadequate $\beta$-cell response to the fall in insulin sensitivity. The fall in insulin sensitivity was not associated with changes in body fat, visceral fat, IGF-I, androgens, or estradiol ${ }^{16}$. In addition, Goran et al. report on a longitudinal study on deterioration of insulin sensitivity and beta-cell function in overweight Hispanic children during pubertal transition ${ }^{15}$. They examined 1-year changes in insulin dynamics in overweight Hispanic children at high-risk of type 2 diabetes as a function of body composition and pubertal transition. They report an increase in fat mass increased by $13 \%(3.0 \mathrm{~kg})$ and a decline of insulin sensitivity by $24 \%$. The fall in insulin sensitivity over 1 year remained highly significant even after adjusting for baseline fat mass, age, gender and change in fat mass. The fall in insulin sensitivity was not significantly influenced by Tanner stage. However, subjects in earlier maturation showed compensatory appropriate $\beta$-cell compensation, whereas subjects in the latter stages of maturation did not. They conclude that failure to increase the acute insulin response in response to the fall in insulin sensitivity may be one factor in the pathogenesis of the progression of paediatric NIDDM. Taken together, interventions to prevent a persistent insulin resistance during puberty are necessary.

\section{Development of overweight and obesity during puberty}

Overweight and obesity are major components determining if insulin resistance will be transient or persistent after puberty. Therefore, it is necessary to start treatment and prevention of this chronic disease as early as possible during childhood and puberty. Possible determinants of overweight and obesity include a rapid catch-up growth after birth to the first birthday, as well as parental influences i.e. overweight of the father and dietary restrained eating behaviour of the mother ${ }^{17-19}$. Later in life, lifestyle factors of the adolescents themselves, such as restrained eating behaviour and physical activity habits become more important ${ }^{18-22}$. As for transient insulin resistance, hormonal changes during puberty may also imply a risk for overweight and obesity.

These changes encompass changes in gonadotropic hormone concentrations, but also leptin concentrations ${ }^{23}$. In girls, a peak in leptin concentrations, observed in Tanner stage 2, precedes a peak in lutein hormone (LH) and follicle stimulating hormone (FSH) concentrations in Tanner stage $3^{23,24}$. Both in boys and girls, the leptin/FM ratio decreases from Tanner stage 2 onwards. In boys, this decrease continues throughout puberty, while in girls, this ratio rebounds again in Tanner stage $5^{23,24}$. These observations imply that during puberty factors independent of fat mass become more important in the regulation of plasma leptin concentrations. The relationship between leptin and gonadotropic hormones during puberty is sex specific with respect to function and timing ${ }^{24}$. Leptin has been shown to be essential in reproductive functioning in both boys and girls ${ }^{24}$. In girls, leptin is 
suggested to act as a permissive factor for the onset of puberty. Several studies have shown that a deficiency in leptin in anorexia nervosa, or hyperleptinaemia in morbid obesity during puberty and as evidenced by studies demonstrating a disturbance of onset as well as progression of puberty in leptin disturbant states like anorexia nervosa or obesity related hyperleptinaemia ${ }^{24}$. With regard to timing, an early leptin peak during puberty, especially in girls, was suggested to contribute to the risk of overweight and obesity ${ }^{23}$.

\section{Changes in sleep duration in relation to overweight and obesity during puberty}

In addition to the sex differences in anthropometric and endocrinological variables seen in the transition from Tanner stage 1 to 5 , a significant reduction in sleep duration is observed ${ }^{25-27}$. Pubertal status appears to be inversely associated with sleep duration ${ }^{25-27}$. Studies on the relationship between sleep duration and body-weight in adolescents report a consistent inverse association between habitual sleep duration and body-weight development ${ }^{25,27-29}$. This relationship remains independent of baseline BMI at the start of puberty, fat mass and obesity-associated (FTO) allele genotype (rs9939609), parent BMI, as well as changes in Baecke scores and hours television viewing during the progressive Tanner stages. However, due to the parallel development it is impossible to disentangle cause and consequence between the variables. Puberty is initiated through pulsatile gonadotropin-releasing hormone $(\mathrm{GnRH})$ release from the hypothalamus and activation of the gonadal axis ${ }^{30,31}$. The subsequent development of secondary sex characteristics originate from shared neuronal systems, with the hypothalamus as integration point ${ }^{32}$. The hypothalamus regulates the sleep-wake and feeding circuits ${ }^{32,33}$. Circuits are connected through the hypocretin-1 hormone that regulates feeding and locomotor activity via the nucleus accumbens, as well as signal transduction on the light-dark cycle to the suprachiasmatic nucleus. Changes in hypothalamic functioning, such as disturbed hypocretin-1 signalling, are associated with disturbance of the circadian cycle and feeding behaviour, affecting energy balance and body composition ${ }^{32,33}$. Consequently, changes in hypothalamic functioning may explain the relationship between the changes in $\mathrm{BMI}$ and in sleep duration during puberty. This hypothesis is underscored by altered hormone concentrations, such as lower leptin concentrations, diminished insulin sensitivity and altered cortisol concentrations in short sleeping adolescents, with the latter significantly contributing to lipogenesis.

\section{Integration of determinants of insulin resistance during puberty}

Thus far we can state that puberty is a vulnerable period in life to develop insulin resistance and obesity. Specific determinants may include sleep characteristics, food intake behaviour, and physical activity.

Sleep is an important factor for normal growth and development during childhood ${ }^{34-39}$. Especially TST (Total Sleeping Time) and QS (Quality Sleep: (Slow Wave Sleep + Rapid Eye Movement sleep)/TST) are crucial sleep factors associated with outcomes on physical, 
cognitive, emotional, and social development in children ${ }^{38}$. Sleep deprivation and poor quality sleep have been identified as independent risk factors for the development of insulin resistance ${ }^{34-37}$. Until now, a limited number of studies on sleep in relation to insulin resistance have been conducted in adolescents ${ }^{40-42}$. For instance, when effects of partial sleep deprivation on markers of glucose metabolism was assessed during 3 consecutive nights of short sleep, acute sleep restriction appeared to reduce insulin sensitivity in adolescent boys ${ }^{41}$. Also, observations on sleep duration and insulin resistance assessed in healthy black and white adolescents show reduced sleep duration associated with increased HOMA-IR. It is suggested that interventions to extend sleep duration may reduce diabetes and obesity risk in youth ${ }^{42}$. A cross-sectional analysis from two examinations conducted in the Cleveland Children's Sleep and Health Cohort ( $n=387 ; 43 \%$ minorities) shows that sleep duration had a quadratic "U-shape" association between sleep duration and HOMA-IR. When adjusted for age, sex, race, preterm status, and activity, adolescents who slept 7.75 hours had the lowest predicted HOMA-IR, while adolescents who slept 5.0 hours or 10.5 hours had HOMAIR indices that were approximately $20 \%$ higher. It was concluded that shorter and longer sleep durations are associated with decreased insulin sensitivity in adolescents ${ }^{40,43}$.

A second target for improving insulin sensitivity directly as well as indirectly by its effects on sleep characteristics concerns the lifestyle factor physical activity. Multiple studies found that increasing physical exercise increased insulin sensitivity in overweight adolescents, even in the absence body weight or fat mass changes ${ }^{44,45}$. Physical exercise improves the insulin sensitivity of important insulin target tissues, such as skeletal muscle, even hours after the exercise has been completed, thereby increasing glucose uptake. Those results support the importance of exercise in all therapies targeting the improvement in insulin resistance and diabetes risk.

As a third component, food intake behaviour is generally accepted as one of the main causes for the development of obesity and metabolic syndrome, with energy intake exceeding energy expenditure leading to increased energy storage in the body. Therefore, treatment of obesity and insulin resistance aims to decrease energy intake and promote healthy food choices ${ }^{46}$. Eating behaviour and eating habits change during childhood, especially in puberty ${ }^{18,19,46}$. This is also the time that many eating disorders, such as binge eating disorder, become apparent, possibly due to issues related to self-esteem and body image. Many studies have found an association between sleep deprivation and food choices ${ }^{47-52}$. Sleep-deprived individuals appear more prone to choose unhealthy foods high in energy and fat content ${ }^{47-52}$. Also, sleep-deprived individuals are reported to have more frequent meals or snacks between meals compared to individuals that had had sufficient sleep ${ }^{49}$. It has been presumed that sleep deprivation is associated with decreased leptin concentrations and increased ghrelin concentrations, thereby promoting the feeling of hunger and suppressing satiety ${ }^{53,54}$. A reduced sleep duration, quality sleep and rapideye movement sleep, or fragmented sleep enhance a positive energy balance through 
altered substrate oxidation, hormone concentrations, sleeping metabolic rate, appetitive behaviours and stress ${ }^{55}$. Circadian misalignment affects sleep architecture and the glucoseinsulin metabolism, substrate oxidation, the HOMA-IR index, leptin concentrations and HPA-axis activity ${ }^{55-58}$.

\section{Associations of sleep characteristics with insulin resistance}

Since very few studies have assessed relationships between sleep duration and insulin resistance during childhood and adolescence, further research is needed to specify these relationships ${ }^{34-43}$. The following study was performed to specify the relationship between sleep characteristics and insulin resistance in overweight and obese adolescents, taking gender, puberty stage, BMI z-score, physical activity and food intake behaviour into account.

\section{MATERIALS AND METHODS}

\section{Subjects}

Subjects were selected from the Centre for Overweight Adolescent and Children's Healthcare $(\mathrm{COACH})$ program in the Netherlands (Maastricht University Medical Centre (MUMC+)). Inclusion criteria were overweight or obesity and age between 10 and 18 years. Exclusion criteria were medical causes of overweight or obesity, such as hypothyroidism. Ethical approval was obtained from the medical ethics committee of the Maastricht University Medical Centre. All parents and children of 12 years and older gave informed consent for participation in $\mathrm{COACH}$ and use of study data for publication.

\section{Study design}

Prior to body-weight treatment, the children and adolescents were subjected to extensive medical screening for causes, risk factors and comorbidities of overweight and obesity during a 24-hour admission at the paediatric ward of the MUMC+. Anthropometric measurements, and blood sampling for insulin sensitivity was performed. Questionnaires on food intake behaviour, physical activity and sleep were completed, and polysomnography took place during an overnight stay in the paediatric ward.

\section{Measurements}

\subsection{Anthropometry}

Body-weight (BW) was measured with the subject in the fasted state, barefoot and in underwear. Weight was determined to the nearest 0.1 kilogram using a calibrated scale (Seca, Chino, CA). Height was measured using a rigid wall-based digital stadiometer (De Grood Metaaltechniek, Nijmegen, the Netherlands) using the Frankfurt plane method ${ }^{59}$. BMI was calculated $\left(\mathrm{BMI}=\right.$ body weight $/$ height $\left.^{2}\right)$. Due to considerable changes of $\mathrm{BMI}$ during 
childhood and growth, the BMI was corrected for age and gender to obtain the BMI z-score ${ }^{60}$. BMI z-scores were calculated with the lambda, mu, sigma (LMS) method in Growth Analyser software (Growth Analyser VE, Rotterdam, the Netherlands). Furthermore, all children were classified as overweight, obese or morbid obese according to the International Obesity Task Force (IOTF) criteria ${ }^{61}$.

\subsection{Puberty}

Puberty stage was determined using the Tanner G/M stages ${ }^{62,63}$. Susman et al. showed that the $95 \%$ confidence interval of mean age in Tanner G/M stage 4 was 14.3 years for girls and 15.0 years for boys ${ }^{64}$. Tanner stages were subsequently divided in three subgroups: prepubertal (Tanner G/M stage 1), peripubertal (Tanner G/M stage 2-3) and postpubertal (Tanner G/M stage 4-5) ${ }^{64-67}$.

\subsection{Insulin sensitivity}

Venous blood sampling was performed to analyse fasting glucose concentration (Cobas 8000 modular analyser, Roche, 154 Woerden, the Netherlands) and fasting insulin concentration (fully automated HPLC Variant II 155 (Bio-Rad Laboratories, Veenendaal, the Netherlands). Insulin sensitivity was assessed by calculating the homeostatic model assessment of insulin resistance (HOMA-IR). HOMA-IR is calculated as glucose (mmol/L) x insulin (pmol/L)/ 22.56.

\subsection{Sleep characteristics}

Sleep was analysed using polysomnography. The polysomnograms yielded the following sleep stages: REM sleep, non-REM sleep stage N1, N2 and N369. A regular night of sleep consists of 4 to 5 cycles of these sleep stages, starting with n-REM stage N1 (drifting off to sleep), followed by N2 (light sleep), N3 (deep sleep) and REM sleep (dreaming sleep). Each sleep stage has a characteristic brain wave pattern, which makes it possible to differentiate each stage using polysomnography ${ }^{69}$.

Furthermore, total sleeping time (TST), defined as total time spent sleeping, was determined. Sleep efficiency (SE) is the total sleeping time divided by the total time spent in bed. REM is the total time spent in rapid eye movement sleep. Slow wave sleep (SWS) is the amount of time spent in non-REM sleep phase N3. Quality sleep (QS) is calculated as REM+SWS)/TST ${ }^{55-58}$.

\subsection{Physical activity}

Physical activity was assessed using a validated Dutch translation of the Baecke questionnaire for children ${ }^{22,70}$. This questionnaire consists of 16 questions analysing three dimensions that were each scored on a five-point scale. The following dimensions were assessed: physical activity at school (school index), physical activity during performing sports (sport index) and physical activity during leisure time (leisure time index). 


\subsection{Food intake behaviour}

Food intake behaviour was assessed by a validated Dutch translation of the Three Factor Eating Questionnaire for children ${ }^{18,19}$. This questionnaire consists of the factors cognitive restraint, disinhibition and hunger. Cognitive restraint refers to the conscious control over food intake. Disinhibition indicates the loss of control in response to disinhibiting stimuli, e.g. stress. Hunger measures the feeling of hunger in general.

\section{Statistical analyses}

Data were analysed using IBM SPSS statistics 19.0 software. Descriptive statistics were given as mean and standard deviation (SD). Factorial ANOVA was used for comparison of means between genders.

Pearson's correlation coefficients were calculated in order to determine associations between insulin sensitivity and sleep parameters, anthropometry, Baecke scores, and food intake behaviour. Significant correlations were further examined using multiple linear regression analysis. Significant results from the regression analyses were combined to assess the association for the combined model. Results were considered significant when p-values were smaller than 0.05 .

\section{RESULTS}

137 children (30.7\% boys; $69.3 \%$ girls) were eligible for this study. Their characteristics are presented in Table 3.1. Fasting glucose concentration, insulin concentration and HOMA-IR are presented as factors of insulin sensitivity. The possible determinants of insulin sensitivity Total Sleeping Time (TST), Quality sleep (QS), Sleep Efficiency (SE), REM sleep and Slow Wave Sleep (SWS), as well as the three dimensions of the Baecke questionnaire (school index, sport index and leisure time index and three dimensions of the TFEQ questionnaire (cognitive restraint, disinhibition and hunger) are also included in Table 2.1.

In the present cohort significant gender related effects were observed with respect to Tanner stages, insulin concentrations, HOMA-IR and scores on the sport index of the Baecke questionnaire (Table 2.1). Furthermore, significant differences were present between puberty stages. In girls, age, BMI z-score and IOTF classification differed significantly between puberty stages. In boys, significant differences between puberty stages were present for insulin concentrations and HOMA-IR. Since significant differences were found for gender and puberty, further multiple regression analyses are presented separately for gender and puberty stage. 


\section{Associations with insulin sensitivity}

In the boys, including all puberty stages, age $\left(r^{2}=0.33, p<0.001\right)$ and puberty stage $\left(r^{2}=0.32\right.$, $\mathrm{p}<0.001$ ) were identified as significant contributors to HOMA-IR (Table 2.2a). The older boys, and those in a later puberty stage, had a higher insulin resistance. There were trends for BMI z-score $\left(r^{2}=0.11, p=0.058\right)$ and Baecke total score $\left(r^{2}=0.12, p=0.078\right)$ to contribute to the explained variance of HOMA-IR, but this did not reach significance. The combination of age, BMI z-score, puberty stage, and Baecke total score explained $55 \%$ of the variance in HOMAIR in boys in this cohort. Age, BMI z-score, and puberty stage were positively, and Baecke score was inversely associated with HOMA-IR.

In the girls, including all puberty stages, BMI z-score was identified as an independent contributor to HOMA-IR $\left(r^{2}=0.07, p=0.018\right)$, explaining $7 \%$ of the variance in HOMA-IR (Table $2.2 b)$. The girls with a higher BMI z-score had a higher insulin resistance. None of the other variables were identified as significant predictors of HOMA-IR in girls in this cohort.

In prepubertal girls, TST $\left(r^{2}=0.59, p=0.02\right)$ was identified as an independent contributor to HOMA-IR, explaining $59 \%$ of variance (Table 3.2c). Also, $\mathrm{SE}\left(\mathrm{r}^{2}=0.58, \mathrm{p}=0.028\right)$ was identified as an independent contributor, explaining $58 \%$ of the variance in HOMA-IR in this group (Table 2.2c). Both were inversely associated with HOMA-IR.

\section{DISCUSSION}

The main research question in this review is to assess the factors affecting insulin resistance during puberty, emphasizing a possible role of sleep characteristics.

Cree-Green et al. showed that insulin resistance is related to an elevated BMI, pubertal hormones along with fat partitioning, elevated free fatty acids, inflammation, and mitochondrial dysfunction ${ }^{14}$. Insulin resistance varies with stage of pubertal development. Normally, adolescents reverse hyperglycaemia postpubertally, once the transient insulin resistance of puberty resolves. Cree-Green et al. propose 4 different phenotypes: in (i) lean children, and in (ii) obese children with no family history of NIDDM and minimal abnormalities in fat partitioning decreased insulin sensitivity is matched by up-regulation of pancreatic insulin secretion ${ }^{14}$. (iii) Obese children with altered fat partitioning do not match decreased insulin sensitivity by pancreatic insulin secretion, leading to IGT or IGF. However, their insulin sensitivity recovers and hyperglycaemia resolves in their postpubertal stage. The transient IGT however may imply that they will be at increased risk for diabetes later in life. (iv) Those children who develop NIDDM did show lower insulin sensitivity together with a decline in $\beta$-cell function resulting in permanent pancreatic insufficiency and hyperglycaemia. Thus, not all children with insulin resistance develop NIDDM. Identifying those early is of major importance for further prevention and treatment. 


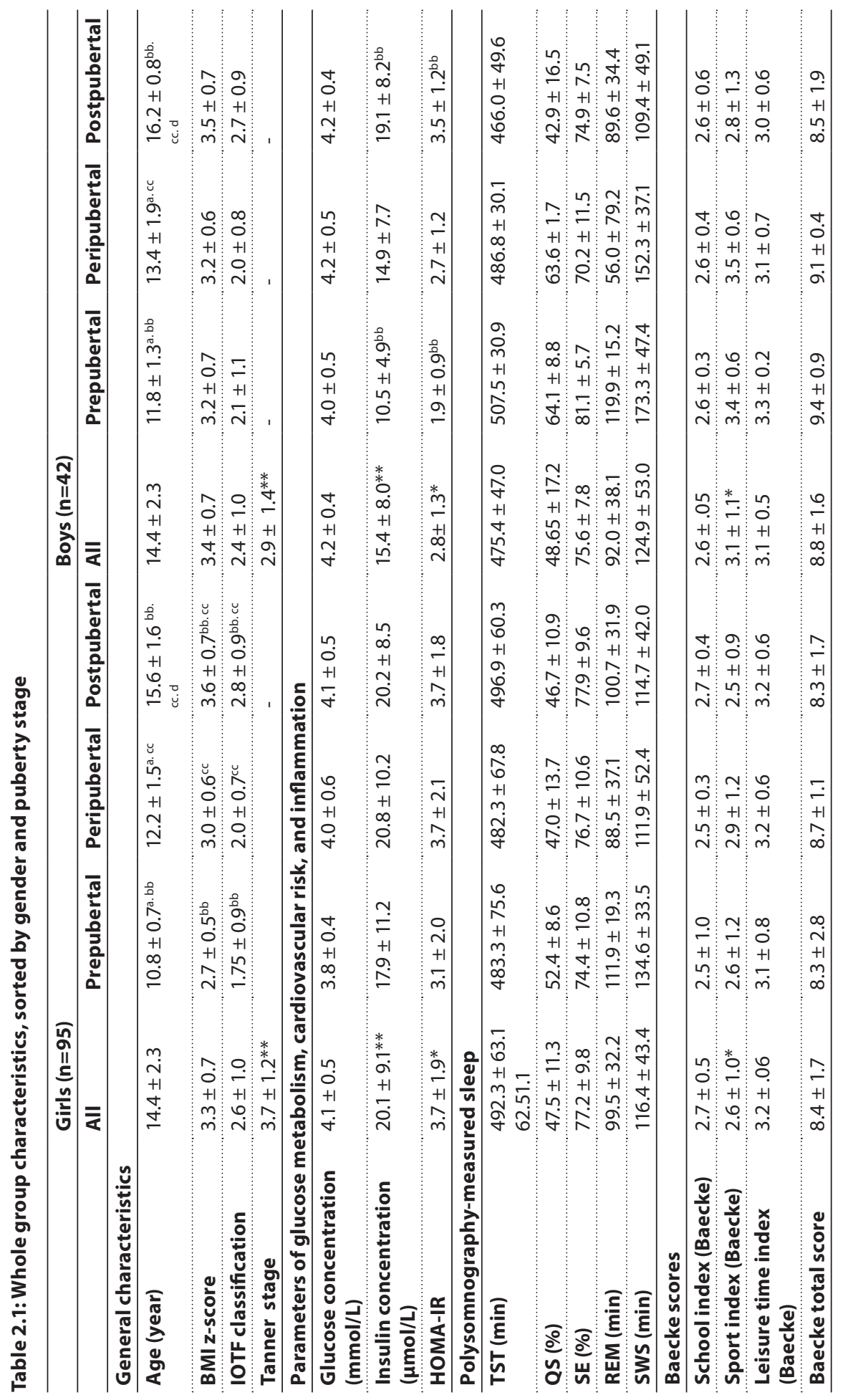




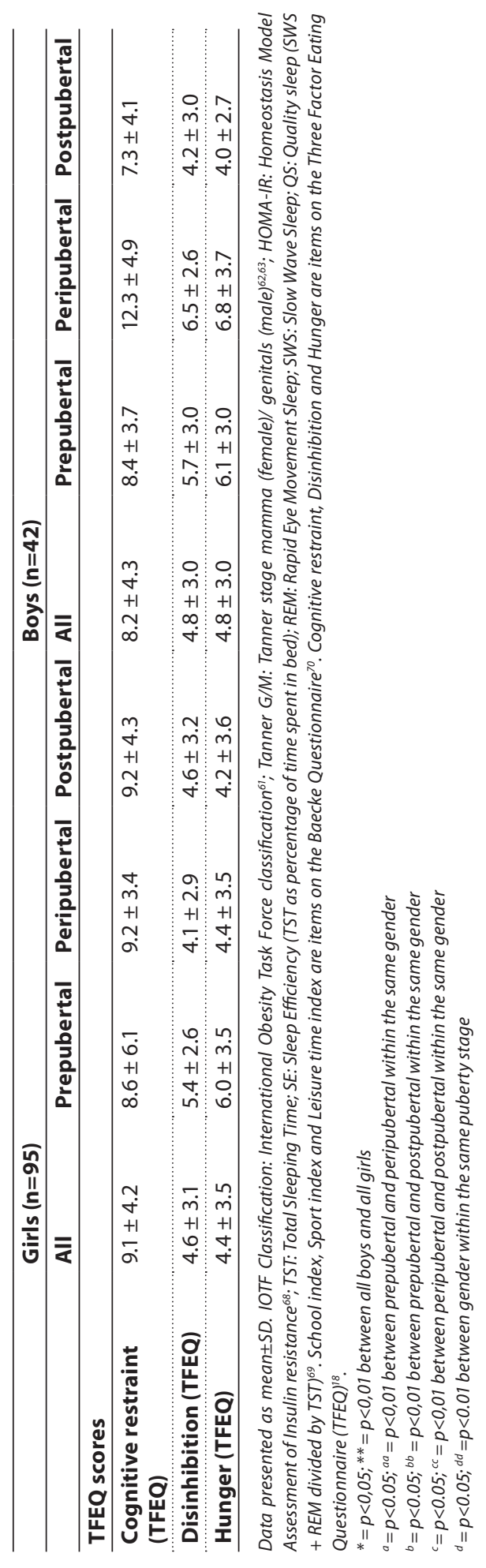



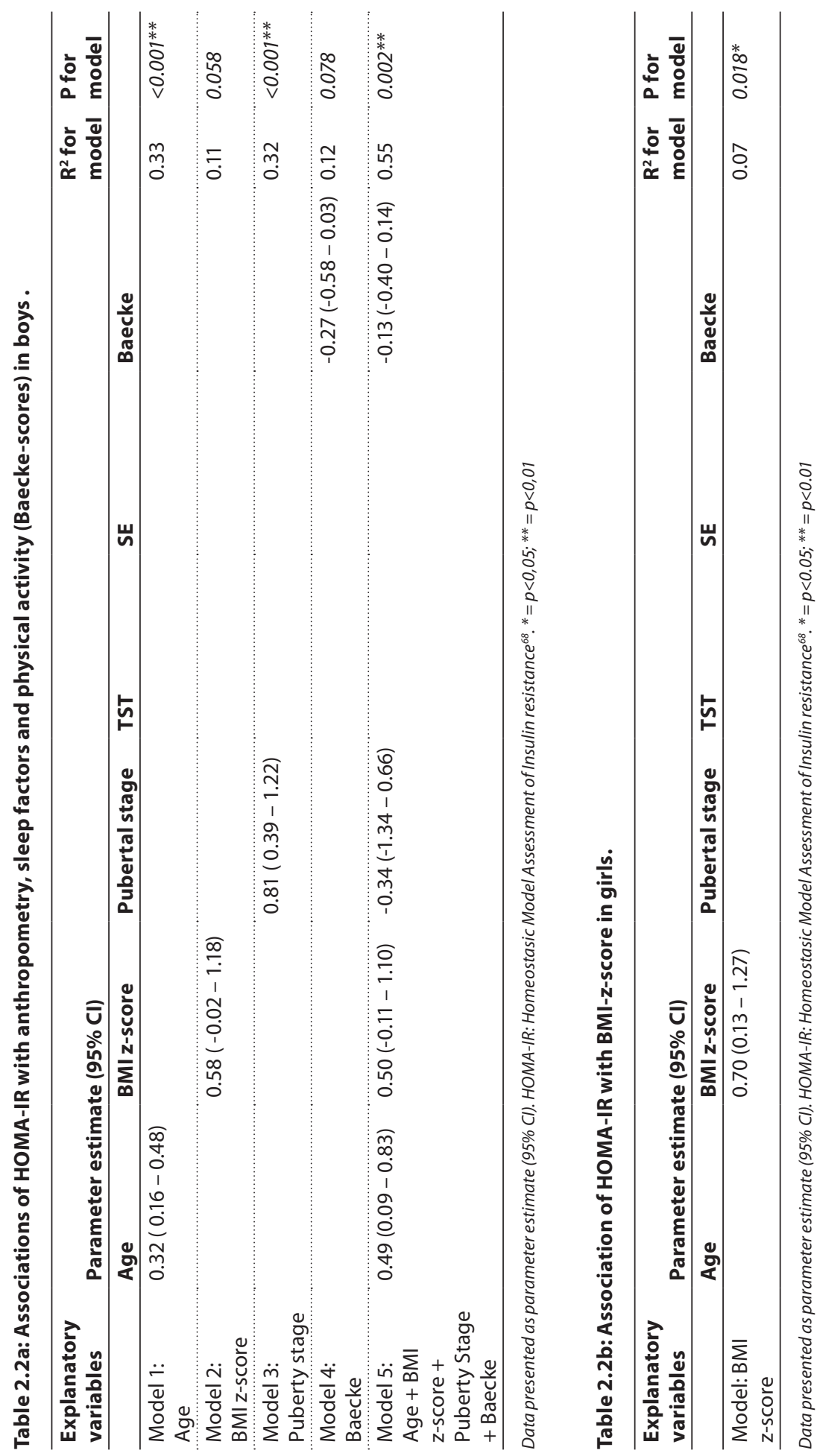


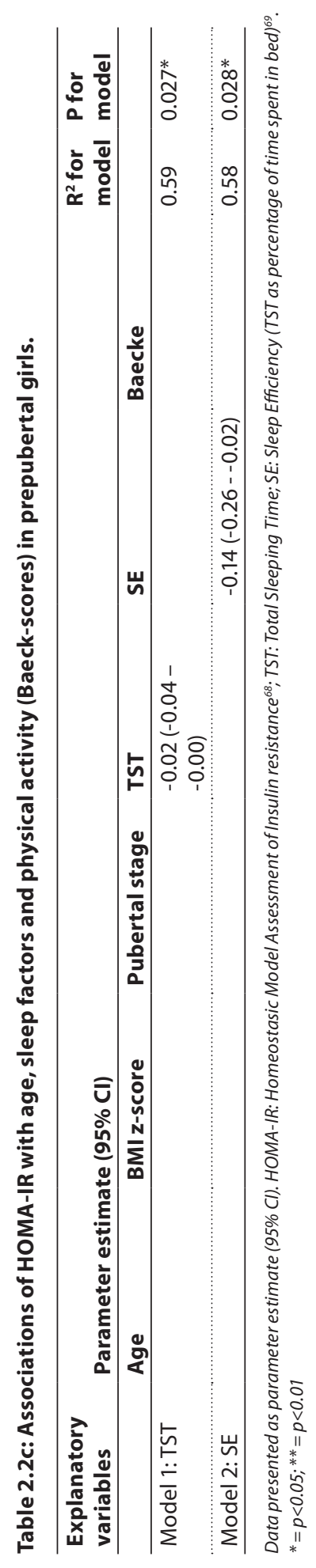


Data from the presented cross-sectional study in overweight and obese adolescent boys and girls at different puberty stages show no transient, yet persistent insulin resistance during the three distinguished puberty stages. In the selected cohort all children and adolescents were admitted based upon their BMI z-scores (Table 3.1). The results are considered separately for gender and puberty stage.

In all girls together, HOMA-IR was positively related to BMI z-score, which is in accordance with present literature ${ }^{7,9,11}$. The postpubertal girls had a higher BMI z-score than the prepubertal and the peripubertal girls, and HOMA-IR remained persistently high postpubertally, in contrast with the usual conditions, where postpubertally HOMA-IR drops again ${ }^{14}$. In all boys together, age and puberty stage were positively associated with HOMA-IR, also confirming previous observations ${ }^{9,11}$. In the boys, postpubertal HOMA-IR was higher compared to the prepubertal figure ${ }^{14}$. We suggest that in the present cohort, the postpubertal boys and girls are at risk for NIDDM, since their postpubertal HOMA-IR is persistently at an elevated level.

The prepubertal girls showed inverse relations of HOMA-IR with total sleeping time and sleep efficiency. In acute studies, both SE and TST have been positively related to insulin sensitivity ${ }^{41,71}$. Longer TST may be a protective factor for glucose tolerance and/or pancreatic $\beta$-cell compensation capacity ${ }^{16}$. This finding is in accordance with published studies that also show that inadequate sleep duration, evaluated by sleep log or actigraphy, was significantly associated with a higher risk for insulin resistance ${ }^{40-42}$ and hyperglycaemia ${ }^{16}$ in children and adolescents.

Strength of this study is the vast collection of measured observational data, including the polysomnography. A limitation is the relatively small cohort, resulting in rather small subgroups. The data of this cross-sectional study on overweight and obese children and adolescents indicate primarily associations of age, BMI z-score, and puberty stage with HOMA-IR, showing no transient, but rather a persistent insulin resistance during puberty. We presume that this is primarily due to the high BMI z-scores of the adolescents. Since most of the associations of insulin resistance with lifestyle factors per puberty stage appeared in the prepubertal girls, it is likely that a prepubertal intervention promoting increase of TST and SE, as well as a reduction of BMI z-score may reduce the risk for persistent insulin resistance. Since all these children and adolescents are at risk for insulin resistance during adulthood, this cohort will be followed up during an intervention aiming to reduce BMI z-score, and longitudinal data will be collected. Another interesting possibility for future research is to assess the effects of diet and physical activity on insulin resistance and sleep architecture in overweight and obese adolescents, with a relatively high HOMA-IR. This is currently being investigated in the 'PREVIEW' (PREVention of diabetes through lifestyle Intervention and population studies in Europe and around the World) study ${ }^{72}$. This study consists of a 3-year randomized control trial (RCT) in 2500 adults and 200 children, where the influence of medium and high protein diets and moderate and high intensity physical 
activity on insulin resistance is being investigated. Furthermore, the PREVIEW study will perform data analyses in a large cohort of studies to assess the most successful combination of diet and physical activity to prevent NIDDM in pre-diabetic adolescents.

In summary, the transient insulin resistance that normally occurs during puberty is at risk to become persistent insulin resistance, especially in obese adolescents with an unfavourable fat-partitioning, and with a family history of NIDDM. Determinants of insulin resistance in adolescents are suggested to be sleeping hours, physical activity and food intake behaviour.

The data presented from a cohort of overweight and obese adolescents at Maastricht University Medical Centre, show an increased risk for NIDDM, indicated by a persistently higher HOMA-IR at each puberty stage. Then the main determinants of HOMA-IR are BMI z-score, age, pubertal stage, and prepubertal sleep efficiency and total sleeping time. The latter is an important clinical finding, as it not only suggests that sleep is a target for preventing insulin sensitivity in overweight youth, but that the sleep interventions should be staged early in puberty to assert maximum results. 


\section{REFERENCES}

1. Wardle J, Brodersen NH, Cole TJ, Jarvis MJ, Boniface DR. Development of adiposity in adolescence: five year longitudinal study of an ethnically and socioeconomically diverse sample of young people in Britain. BMJ (Clinical research ed). 2006;332(7550):1130-1135.

2. Carskadon MA, Acebo C, Jenni OG. Regulation of adolescent sleep: implications for behavior. Annals of the New York Academy of Sciences. 2004;1021:276-291.

3. Colrain IM, Baker FC. Changes in sleep as a function of adolescent development. Neuropsychol Rev. 2011;21(1):5-21.

4. Cusatis DC, Shannon BM. Influences on adolescent eating behavior. The Journal of adolescent health : official publication of the Society for Adolescent Medicine. 1996;18(1):27-34.

5. Cutler GJ, Flood A, Hannan P, Neumark-Sztainer D. Multiple sociodemographic and socioenvironmental characteristics are correlated with major patterns of dietary intake in adolescents. Journal of the American Dietetic Association. 2011;111(2):230-240.

6. Hagenauer $\mathrm{MH}$, Lee TM. Adolescent sleep patterns in humans and laboratory animals. Hormones and behavior. 2013;64(2):270-279.

7. Moran A, Jacobs DR, Jr., Steinberger J, et al. Insulin resistance during puberty: results from clamp studies in 357 children. Diabetes. 1999;48(10):2039-2044.

8. Nader PR, Bradley RH, Houts RM, McRitchie SL, O'Brien M. Moderate-to-vigorous physical activity from ages 9 to 15 years. Jama. 2008;300(3):295-305.

9. Rizzo NS, Ruiz JR, Oja L, Veidebaum T, Sjostrom M. Associations between physical activity, body fat, and insulin resistance (homeostasic model assessment) in adolescents: the European Youth Heart Study. The American journal of clinical nutrition. 2008;87(3):586-592.

10. Levy-Marchal C, Arslanian S, Cutfield W, et al. Insulin resistance in children: consensus, perspective, and future directions. The Journal of clinical endocrinology and metabolism. 2010;95(12):5189-5198.

11. Xu L, Li M, Yin J, et al. Change of Body Composition and Adipokines and Their Relationship with Insulin Resistance across Pubertal Development in Obese and Nonobese Chinese Children: The BCAMS Study. Int J Endocrinol. 2012;2012:389108.

12. Hannon TS, Janosky J, Arslanian SA. Longitudinal study of physiologic insulin resistance and metabolic changes of puberty. Pediatr Res. 2006;60(6):759-763.

13. Jeffery AN, Metcalf BS, Hosking J, Streeter AJ, Voss LD, Wilkin TJ. Age before stage: insulin resistance rises before the onset of puberty: a 9-year longitudinal study (EarlyBird 26). Diabetes Care. 2012;35(3):536-541.

14. Cree-Green M, Triolo TM, Nadeau KJ. Etiology of insulin resistance in youth with type 2 diabetes. Curr Diab Rep. 2013;13(1):81-88.

15. Goran MI, Gower BA. Longitudinal study on pubertal insulin resistance. Diabetes. 2001;50(11): 2444-2450.

16. Goran MI, Shaibi GQ, Weigensberg MJ, Davis JN, Cruz ML. Deterioration of insulin sensitivity and beta-cell function in overweight Hispanic children during pubertal transition: a longitudinal assessment. International journal of pediatric obesity: IJPO: an official journal of the International Association for the Study of Obesity. 2006;1(3):139-145.

17. Fuentes RM, Notkola IL, Shemeikka S, Tuomilehto J, Nissinen A. Tracking of body mass index during childhood: a 15-year prospective population-based family study in eastern Finland. International journal of obesity and related metabolic disorders : journal of the International Association for the Study of Obesity. 2003;27(6):716-721.

18. Stunkard AJ, Messick $S$. The three-factor eating questionnaire to measure dietary restraint, disinhibition and hunger. J Psychosom Res. 1985;29(1):71-83.

19. Vogels N, Posthumus DL, Mariman EC, et al. Determinants of overweight in a cohort of Dutch children. The American journal of clinical nutrition. 2006;84(4):717-724. 
20. Ekelund U, Aman J, Yngve A, Renman C, Westerterp K, Sjostrom M. Physical activity but not energy expenditure is reduced in obese adolescents: a case-control study. The American journal of clinical nutrition. 2002;76(5):935-941.

21. Hoos MB, Gerver WJ, Kester AD, Westerterp KR. Physical activity levels in children and adolescents. Int J Obes Relat Metab Disord. 2003;27(5):605-609.

22. Vogels N, Westerterp KR, Posthumus DL, Rutters F, Westerterp-Plantenga MS. Daily physical activity counts vs structured activity counts in lean and overweight Dutch children. Physiol Behav. 2007;92(4):611-616.

23. Rutters F, Nieuwenhuizen AG, Verhoef SP, Lemmens SG, Vogels N, Westerterp-Plantenga MS. The relationship between leptin, gonadotropic hormones, and body composition during puberty in a Dutch children cohort. European journal of endocrinology / European Federation of Endocrine Societies. 2009;160(6):973-978.

24. Bluher S, Mantzoros CS. Leptin in reproduction. Current opinion in endocrinology, diabetes, and obesity. 2007;14(6):458-464.

25. Knutson KL. The association between pubertal status and sleep duration and quality among a nationally representative sample of U. S. adolescents. Am J Hum Biol. 2005;17(4):418-424.

26. Rutters F, Gerver WJ, Nieuwenhuizen AG, Verhoef SP, Westerterp-Plantenga MS. Sleep duration and body-weight development during puberty in a Dutch children cohort. International journal of obesity (2005). 2010;34(10):1508-1514.

27. Thorleifsdottir B, Bjornsson JK, Benediktsdottir B, Gislason T, Kristbjarnarson H. Sleep and sleep habits from childhood to young adulthood over a 10-year period. J Psychosom Res. 2002;53(1):529-537.

28. Lumeng JC, Somashekar D, Appugliese D, Kaciroti N, Corwyn RF, Bradley RH. Shorter sleep duration is associated with increased risk for being overweight at ages 9 to 12 years. Pediatrics. 2007;120(5):1020-1029.

29. Rutters F, Gerver WJ, Nieuwenhuizen AG, Verhoef SPM, Westerterp-Plantenga MS. Sleep duration and body-weight development during puberty in a Dutch children cohort. International Journal Of Obesity. 2010;34:1508.

30. DiVall SA, Radovick S. Endocrinology of female puberty. Current opinion in endocrinology, diabetes, and obesity. 2009;16(1):1-4.

31. Lewis K, Lee PA. Endocrinology of male puberty. Current opinion in endocrinology, diabetes, and obesity. 2009;16(1):5-9.

32. Adamantidis A, de Lecea L. Sleep and metabolism: shared circuits, new connections. Trends in endocrinology and metabolism: TEM. 2008;19(10):362-370.

33. Vanitallie TB. Sleep and energy balance: Interactive homeostatic systems. Metabolism: clinical and experimental. 2006;55(10 Suppl 2):S30-35.

34. Mesarwi O, Polak J, Jun J, Polotsky VY. Sleep disorders and the development of insulin resistance and obesity. Endocrinol Metab Clin North Am. 2013;42(3):617-634.

35. Nixon GM, Thompson JM, Han DY, et al. Short sleep duration in middle childhood: risk factors and consequences. Sleep. 2008;31(1):71-78.

36. Spiegel K, Leproult R, Van Cauter E. Impact of sleep debt on metabolic and endocrine function. Lancet. 1999;354(9188):1435-1439.

37. Van Cauter E. Sleep disturbances and insulin resistance. Diabetic medicine : a journal of the British Diabetic Association. 2011;28(12):1455-1462.

38. Matricciani L, Blunden S, Rigney G, Williams MT, Olds TS. Children's sleep needs: is there sufficient evidence to recommend optimal sleep for children? Sleep. 2013;36(4):527-534.

39. de Jong E, Stocks T, Visscher TL, HiraSing RA, Seidell JC, Renders CM. Association between sleep duration and overweight: the importance of parenting. International journal of obesity (2005). 2012;36(10):1278-1284. 
40. Javaheri S, Storfer-Isser A, Rosen CL, Redline S. Association of short and long sleep durations with insulin sensitivity in adolescents. The Journal of pediatrics. 2011;158(4):617-623.

41. Klingenberg L, Chaput JP, Holmback U, et al. Acute Sleep Restriction Reduces Insulin Sensitivity in Adolescent Boys. Sleep. 2013;36(7):1085-1090.

42. Matthews KA, Dahl RE, Owens JF, Lee L, Hall M. Sleep duration and insulin resistance in healthy black and white adolescents. Sleep. 2012;35(10):1353-1358.

43. Rey-Lopez JP, de Carvalho HB, de Moraes AC, et al. Sleep time and cardiovascular risk factors in adolescents: the HELENA (Healthy Lifestyle in Europe by Nutrition in Adolescence) study. Sleep Med. 2014;15(1):104-110.

44. Fedewa MV, Gist NH, Evans EM, Dishman RK. Exercise and insulin resistance in youth: a metaanalysis. Pediatrics. 2014;133(1):e163-174.

45. Tompkins CL, Moran K, Preedom S, Brock DW. Physical activity-induced improvements in markers of insulin resistance in overweight and obese children and adolescents. Current diabetes reviews. 2011;7(3):164-170.

46. Chapman CD, Benedict C, Brooks SJ, Schioth HB. Lifestyle determinants of the drive to eat: a meta-analysis. The American journal of clinical nutrition. 2012;96(3):492-497.

47. Ekmekcioglu C, Touitou Y. Chronobiological aspects of food intake and metabolism and their relevance on energy balance and weight regulation. Obesity reviews : an official journal of the International Association for the Study of Obesity. 2011;12(1):14-25.

48. Hart CN, Carskadon MA, Considine RV, et al. Changes in children's sleep duration on food intake, weight, and leptin. Pediatrics. 2013;132(6):e1473-1480.

49. Hicks RA, McTighe $S$, Juarez M. Sleep duration and eating behaviors of college students. Perceptual and motor skills. 1986;62(1):25-26.

50. Hogenkamp PS, Nilsson E, Nilsson VC, et al. Acute sleep deprivation increases portion size and affects food choice in young men. Psychoneuroendocrinology. 2013;38(9):1668-1674.

51. St-Onge MP. The role of sleep duration in the regulation of energy balance: effects on energy intakes and expenditure. Journal of clinical sleep medicine : JCSM : official publication of the American Academy of Sleep Medicine. 2013;9(1):73-80.

52. Wells TT CD. Effect of partial sleep deprivation on food consumption and food choice. Psychology and Health. 2006;21(1):79-86.

53. Leproult R, Copinschi G, Buxton O, Van Cauter E. Sleep loss results in an elevation of cortisol levels the next evening. Sleep. 1997;20(10):865-870.

54. St-Onge MP, O'Keeffe M, Roberts AL, RoyChoudhury A, Laferrere B. Short sleep duration, glucose dysregulation and hormonal regulation of appetite in men and women. Sleep. 2012;35(11):1503-1510.

55. Gonnissen HK, Hulshof T, Westerterp-Plantenga MS. Chronobiology, endocrinology, and energy- and food-reward homeostasis. Obesity reviews : an official journal of the International Association for the Study of Obesity. 2013;14(5):405-416.

56. Gonnissen HK, Hursel R, Rutters F, Martens EA, Westerterp-Plantenga MS. Effects of sleep fragmentation on appetite and related hormone concentrations over $24 \mathrm{~h}$ in healthy men. The British journal of nutrition. 2013;109(4):748-756.

57. Gonnissen HK, Mazuy C, Rutters F, Martens EA, Adam TC, Westerterp-Plantenga MS. Sleep architecture when sleeping at an unusual circadian time and associations with insulin sensitivity. PloS one. 2013;8(8):e72877.

58. Gonnissen HK, Rutters F, Mazuy C, Martens EA, Adam TC, Westerterp-Plantenga MS. Effect of a phase advance and phase delay of the 24-h cycle on energy metabolism, appetite, and related hormones. The American journal of clinical nutrition. 2012;96(4):689-697.

59. Kean CFAMMR. Natural head position, a basic consideration in the interpretation of cephalometric radiographs. Am J Phys Antropol. 1958;16(2):213 - 234.

60. Must A, Anderson SE. Body mass index in children and adolescents: considerations for population-based applications. International journal of obesity (2005). 2006;30(4):590-594. 
61. Cole TJ, Bellizzi MC, Flegal KM, Dietz WH. Establishing a standard definition for child overweight and obesity worldwide: international survey. BMJ (Clinical research ed). 2000;320(7244):12401243.

62. Marshall WA, Tanner JM. Variations in pattern of pubertal changes in girls. Arch Dis Child. 1969;44(235):291-303.

63. Marshall WA, Tanner JM. Variations in the pattern of pubertal changes in boys. Arch Dis Child. 1970;45(239):13-23.

64. Susman EJ, Houts RM, Steinberg L, et al. Longitudinal development of secondary sexual characteristics in girls and boys between ages $91 / 2$ and 151/2 years. Archives of pediatrics \& adolescent medicine. 2010;164(2):166-173.

65. Macdonald HM, Kontulainen SA, Mackelvie-O'Brien KJ, et al. Maturity- and sex-related changes in tibial bone geometry, strength and bone-muscle strength indices during growth: a 20-month pQCT study. Bone. 2005;36(6):1003-1011.

66. Nishiyama KK, Macdonald HM, Moore SA, Fung T, Boyd SK, McKay HA. Cortical porosity is higher in boys compared with girls at the distal radius and distal tibia during pubertal growth: an HR-pQCT study. Journal of bone and mineral research : the official journal of the American Society for Bone and Mineral Research. 2012;27(2):273-282.

67. Wang Q, Wang XF, luliano-Burns S, Ghasem-Zadeh A, Zebaze R, Seeman E. Rapid growth produces transient cortical weakness: a risk factor for metaphyseal fractures during puberty. Journal of bone and mineral research : the official journal of the American Society for Bone and Mineral Research. 2010;25(7):1521-1526.

68. Matthews DR, Hosker JP, Rudenski AS, Naylor BA, Treacher DF, Turner RC. Homeostasic model assessment: insulin resistance and beta-cell function from fasting plasma glucose and insulin concentrations in man. Diabetologia. 1985;28(7):412-419.

69. Berry RB, Budhiraja R, Gottlieb DJ, et al. Rules for scoring respiratory events in sleep: update of the 2007 AASM Manual for the Scoring of Sleep and Associated Events. Deliberations of the Sleep Apnea Definitions Task Force of the American Academy of Sleep Medicine. J Clin Sleep Med. 2012;8(5):597-619.

70. Baecke JA, Burema J, Frijters JE. A short questionnaire for the measurement of habitual physical activity in epidemiological studies. The American journal of clinical nutrition. 1982;36(5):936942.

71. Zhu Y, Li AM, Au CT, et al. Association between sleep architecture and glucose tolerance in children and adolescents. J Diabetes. 2015;7(1):10-15.

72. Raben A FM, Feskens E, Westerterp-Plantenga MS, Schlicht W, Brand-Miller J. PREVIEW: PREVention of diabetes through lifestyle Intervention and population studies in Europe and around the World. On behalf of the PREVIEW consortium. Obes Facts. 2013;6:194. 


\title{
Chapter 3
}

PREVIEW: Prevention of diabetes through lifestyle intervention in a multicentre study in Europe in children (10-17y). Design, methods, and baseline results

\author{
Elke Dorenbos \\ Mathijs Drummen \\ Jesse Rijks \\ Tanja Adam \\ Pauline Stouthart \\ J. Alfredo Martínez \\ Santiago Navas-Carretero \\ Gareth Stratton \\ Nils Swindell \\ Mikael Fogelholm \\ Anne Raben \\ Margriet Westerterp-Plantenga \\ Anita Vreugdenhil
}




\begin{abstract}
Insulin resistance (IR) in adolescence is associated with T2DM. The PREVIEW study assesses the effectiveness of a high-protein, low-glycaemic index diet and moderate-protein, moderateglycaemic index diet to decrease IR in insulin resistant adolescents with overweight/ obesity. Inclusion criteria were age 10-17y, HOMA-IR $\geq 2.0$ and overweight/obesity. In 126 adolescents (13.6 $\pm 2.2 y$, BMI z-score 3.04 \pm 0.66 , HOMA-IR 3.48 \pm 2.28 ) anthropometrics, fat mass percentage (FM\%), metabolic parameters, physical activity, food intake and sleep were measured. Baseline characteristics did not differ between the groups. IR was higher in pubertal adolescents with morbid obesity than in prepubertal adolescents with morbid obesity $(5.41 \pm 1.86$ vs. $3.23 \pm 1.86, p=0.007)$ and prepubertal and pubertal adolescents with overweight/obesity (vs. $3.61 \pm 1.60, p=0.004$ and vs. $3.40 \pm 1.50, p<0.001$ respectively). IR was associated with sex, Tanner stage, BMI z-score, and FM\%. Fasting glucose concentrations were negatively associated with Baecke Sport $(r=-0.223, p=0.025)$ and positively with daytime sleepiness $(r=0.280, p=0.016)$ independent of sex, Tanner stage, BMI $z$-score and $\mathrm{FM} \%$. In conclusion, IR was most severe in pubertal adolescents with morbid obesity. The relations between fasting glucose concentration with Baecke Sport and sleepiness suggest these might be possible targets for diabetes prevention.
\end{abstract}




\section{INTRODUCTION}

Up to $52 \%$ of children and adolescents with overweight present with insulin resistance (IR), a precursor of T2DM'. IR is defined as the inability of insulin to increase glucose uptake and utilization, leading to a compensatory increase in insulin secretion to maintain normal blood glucose values. IR is associated with metabolic disturbances, non-alcoholic fatty liver disease (NAFLD) and development of cardiovascular disorders. IR in adolescents is further complicated by a physiological transient insulin resistance during puberty, which seems to be more severe in adolescents with overweight and obesity ${ }^{2-4}$. Some adolescents appear to have insufficient $\beta$-cell insulin secretion to compensate for the increased IR, which puts them at risk for $\beta$-cell exhaustion and, ultimately, T2DM ${ }^{4}$. Thus, puberty may be a critical time for diabetes prevention in insulin resistant adolescents with overweight and obesity.

Previous studies have shown that weight loss improves IR in children and adolescents with overweight and obesity ${ }^{8}$. Diets higher in protein, especially when combined with lower glycaemic index $(\mathrm{Gl})$, have shown to be protective for obesity in children'. However, it is not known which dietary strategy is most effective for IR reduction, independent of weight change, in high-risk insulin resistant adolescents. The PREVention of diabetes through Lifestyle Intervention and population studies in Europe and around the World (PREVIEW) study aims to assess the effectiveness of two dietary strategies on reducing IR, independent of weight change, in insulin resistant adolescents at high risk for T2DM. Participants were randomized into a moderate-protein, moderate-Gl diet, following clinical standards for diabetes prevention, or a high-protein, low-Gl diet in line with the most successful diet for adolescents in the DiOGenes study ${ }^{9}$. In this paper the study design, methods and baseline results are presented.

\section{MATERIALS AND METHODS}

Here we present a concise version of the methods. More information on the study protocol is presented in the Supplement.

\section{Participants}

The PREVIEW study in adolescents is a randomized trial conducted at three study sites (Maastricht University, the Netherlands; University of Navarra, Spain; and Swansea University, United Kingdom). Inclusion criteria included age 10-17 years, overweight or (morbid) obesity, IR (HOMA-IR $\geq 2.0$ for adolescents with Tanner stages $\geq 3$ or any HOMA-IR for adolescents with Tanner stages 1-2), and written informed consent by both parents and adolescents aged $\geq 12$ years. Adolescents were excluded from participation in the presence of medical conditions or medication use that might compromise study outcomes (e.g. T2DM, bariatric 
surgery or metformin use) and issues leading to difficulty in compliance with the protocol (e.g. severe food intolerances).

\section{Design and intervention}

The first 8 weeks of the study were aimed at weight stabilization in spite of growth. Participants received a personalized menu following the recommended guidelines of 15/55/30 energy percentage (En\%) protein/carbohydrate/fat. In the 96-weeks intervention phase adolescents were randomized into a moderate-protein moderate-GI arm (MP) consisting of a target macronutrient composition of 15/55/30En\% protein/carbohydrate/ fat, and a Gl $\geq 56$, or a high-protein, low-Gl arm (HP) consisting of 25/45/30En\% protein/ carbohydrate/fat and a Gl $\leq 50$ (Supplementary Table 4.1). Adolescents received personalized menus and recipes in line with their randomization arm and energy needs. All participants received instructions on both high-intensity and moderate-intensity physical activity (PA) and exercise in general was encouraged. Nutritional and PA counselling was provided at each visit to improve compliance. The study protocol was approved by local Medical Ethics Committees and was compliant with the Declaration of Helsinki and the ICH-GCP. The trial was registered on ClinicalTrials.gov (number NCT01777893).

\section{Measurements}

Measurements were performed during standardized clinical investigation days (CIDs) (Supplementary Table 3.2).

\subsection{Anthropometric measurements and body composition}

Height and weight were measured while adolescents were in fasted state, barefoot, and wearing only underwear. Subsequently body mass index (BMI), age- and sex-specific BMI z-scores and overweight classifications were calculated ${ }^{7}$. Body composition was measured using air-displacement plethysmography or bio-impedance measurements. Adolescents were classified as prepubertal (Tanner genital/mammae stages 1-2) or pubertal (Tanner stages $3-5)^{5,6}$.

\subsection{Parameters of glucose metabolism, lipids, inflammation, and liver parameters and blood} pressure

After an overnight fast, a blood sample was taken to assess glucose metabolism parameters, lipids, markers of inflammation and liver parameters (Table 3.1 \& Supplementary Table 3.2). Insulin sensitivity was assessed by Homeostatic Model Assessment of Insulin Resistance (HOMA-IR; glucose $(\mathrm{mmol} / \mathrm{L}){ }^{*}$ insulin $\left.(\mathrm{mU} / \mathrm{L}) / 22,5\right)^{10}$. Adolescents were defined as insulin resistant when $H O M A-I R \geq 2.0$ or any $H O M A-I R$ for prepubertal adolescents due to a physiologically lower HOMA-IR in early puberty ${ }^{3}$. An average of three blood pressure measurements was used for analyses. 


\subsection{Lifestyle factors: food intake behaviour, physical activity and sleep}

Adolescents completed a 4-day food record to assess energy intake, macronutrient composition, glycaemic index and glycaemic load (GL). The three Factor Eating Questionnaire (TFEQ) adapted for adolescents was used to assess food intake behaviour ${ }^{11}$. In a subcohort at UM 24h urinary nitrogen was obtained to calculate protein intake. PA was measured by 7-day accelerometry using an Actigraph (Actigraph GT3X accelerometer) and by the Baecke questionnaire adapted for adolescents ${ }^{14}$. The Pittsburgh Sleep Quality Index (PSQI) and Epworth Sleepiness Scale (ESS) were used to assess self-reported sleep parameters ${ }^{12,13}$. In a subcohort sleep was measured using polysomnography.

\section{Statistical analyses}

In future analyses, effects of the two dietary arms will be compared using ANOVA repeated measures with diet as a factor. Multiple regression analyses will be used to identify the contribution of different variables to HOMA-IR change. Baseline analyses in this paper were performed using the Statistical Package for the Social Sciences (SPSS) 24.0 (SPSS Inc, New York). ANOVA or Mann-Whitney-U test were used for assessing differences between the two intervention groups, depending on normality of data. Associations between parameters were assessed using Pearson's or Spearman's correlation coefficients, which were corrected for relevant variables. A p-value $<0.05$ was considered statistically significant.

\section{RESULTS}

126 adolescents that completed screening and body composition measurement were included in baseline analyses (58.7\% girls, age 13.6 $\pm 2.2 \mathrm{y}$, BMI z-score 3.04 \pm 0.66 , HOMAIR 3.35 $\pm 1.80,31.0 / 46.0 / 23.0 \%$ overweight/obesity/morbid obesity, Figure 3.1 and Table 3.1). Participant characteristics were not significantly different between the HP and MP groups. HOMA-IR distribution in high-risk adolescents (HOMA-IR $\geq 2.0)$ did not differ between adolescents in different Tanner stages or between the sexes (Supplementary Figure 3.1). IR was higher in pubertal adolescents with morbid obesity than in prepubertal adolescents with morbid obesity ( $5.41 \pm 1.86$ vs. $3.23 \pm 1.86, \mathrm{p}=0.007)$, and prepubertal and pubertal adolescents with overweight/obesity (vs. 3.61 $\pm 1.60, p=0.004$ and $3.40 \pm 1.50, p<0.001$ respectively).

Parameters of glucose metabolism were positively associated with sex, Tanner stage, BMI z-score, fat mass index, fat free mass index and fat mass percentage. Independently of sex, Tanner stage, BMI z-score and FM\%, Baecke Sport score was inversely $(r=-0.223$, $p=0.025)$ and ESS daytime sleepiness positively $(r=0.280, p=0.016)$ associated with fasting blood glucose concentrations (Supplementary Table 3.3). 


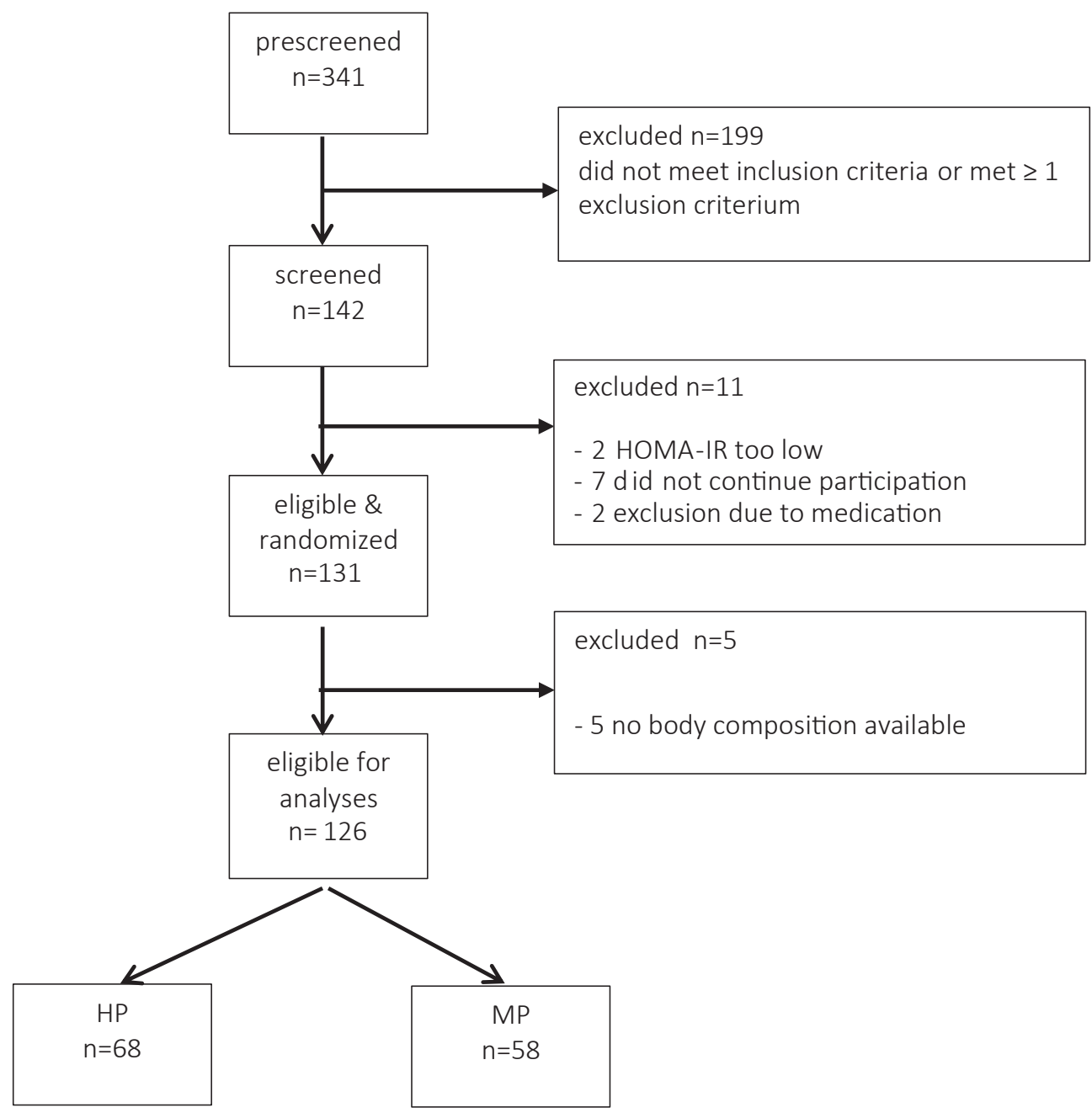

Figure 3.1: Flowchart of participants in the PREVIEW study in adolescents

Drop-out analyses showed no differences between adolescents that completed the PA and food intake questionnaires and adolescents that did not. Adolescents that completed sleep questionnaires had a higher fat free mass index (19.0 $\pm 2.6 \mathrm{vs} .17 .3 \pm 2.4 \mathrm{~kg} / \mathrm{m} 2, \mathrm{p}=0.001)$, lower FM\% (37.1 \pm 9.3 vs. $40.4 \pm 7.1 \%, \mathrm{p}=0.027$ ) and higher fasting blood glucose concentration $(4.8 \pm 0.7$ vs. $4.4 \pm 0.7 \mathrm{mmol} / \mathrm{L}, \mathrm{p}=0.007)$ than adolescents that did not complete these questionnaires (Supplementary Table 3.4). 


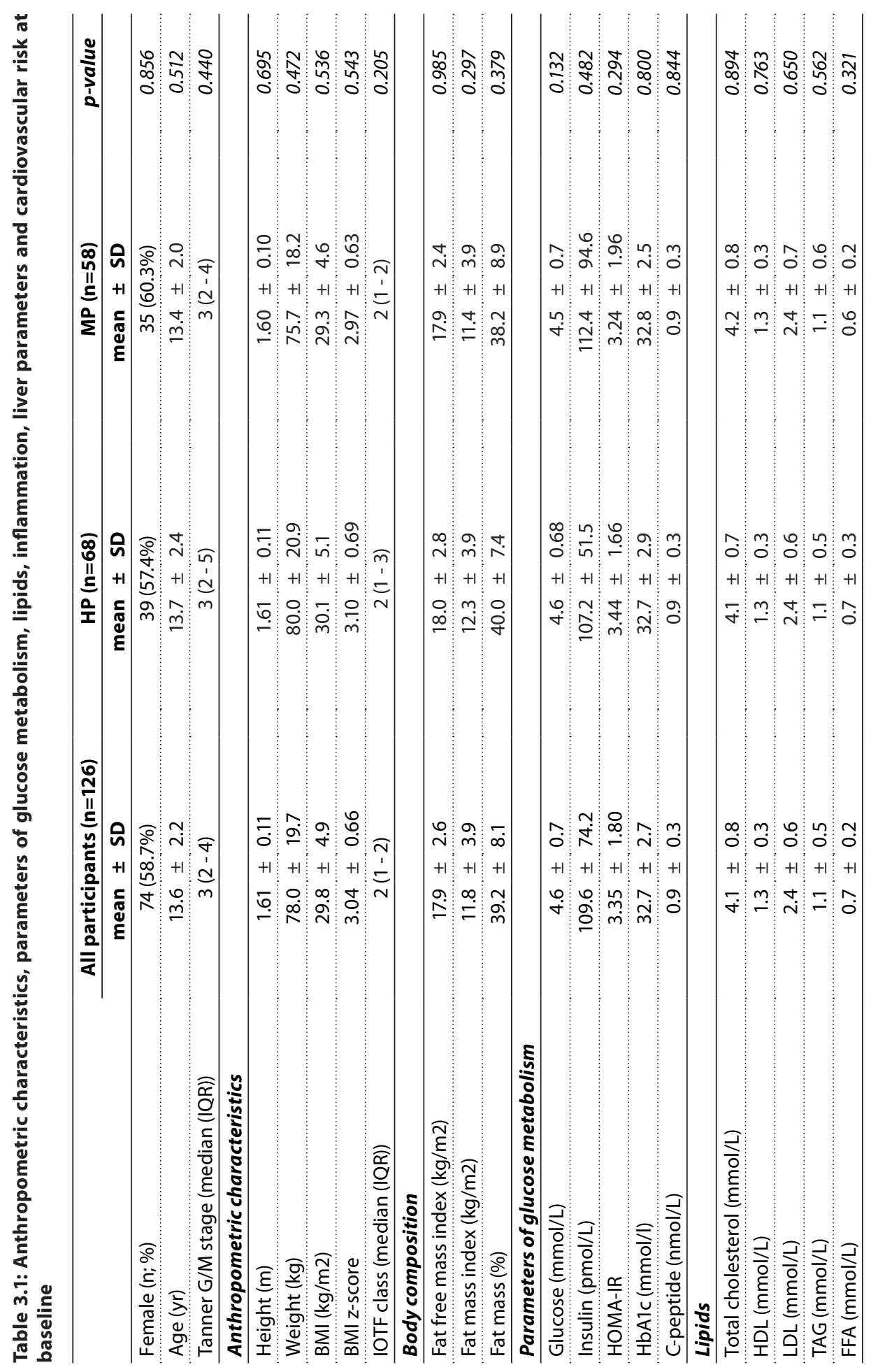




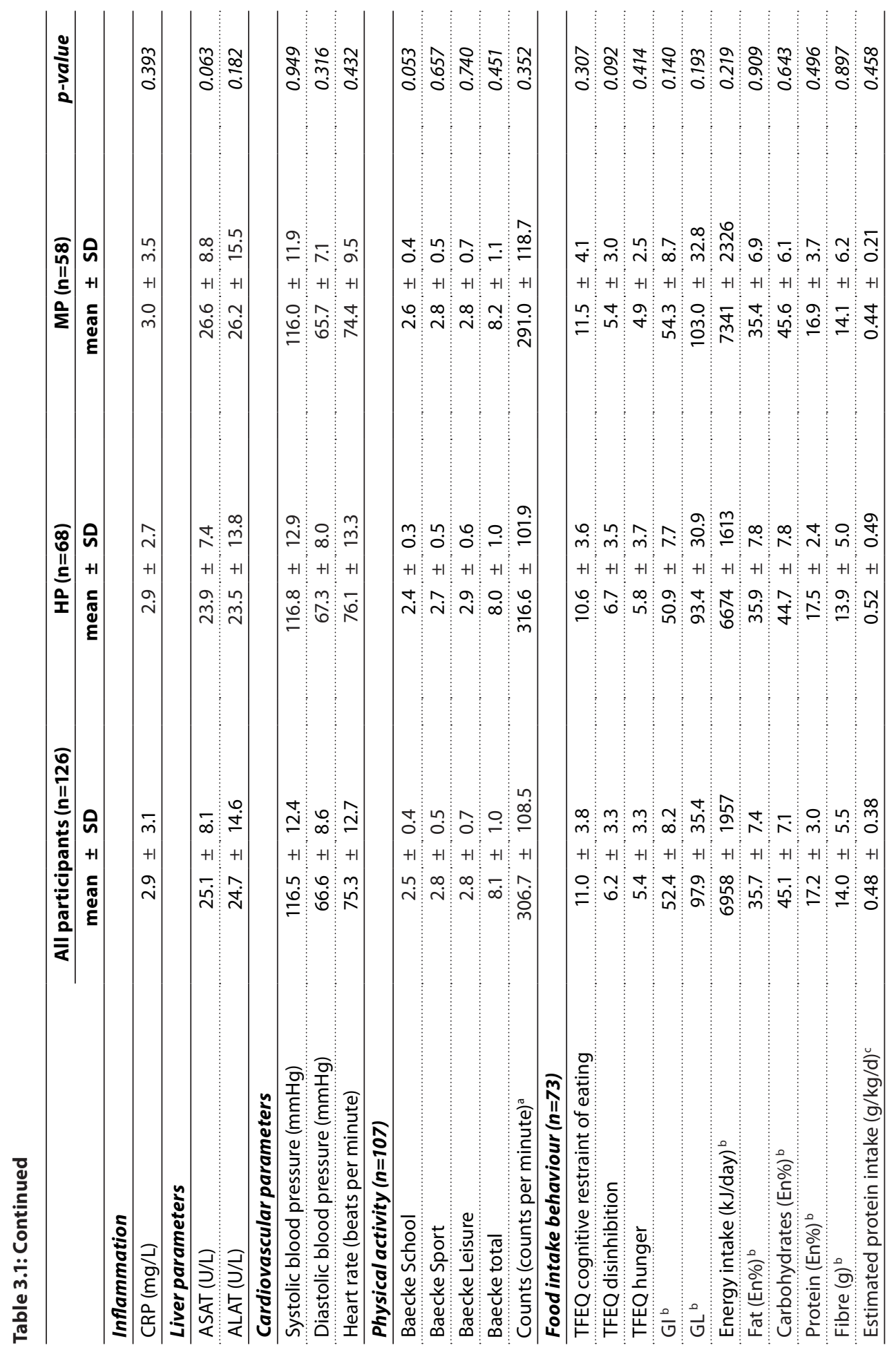




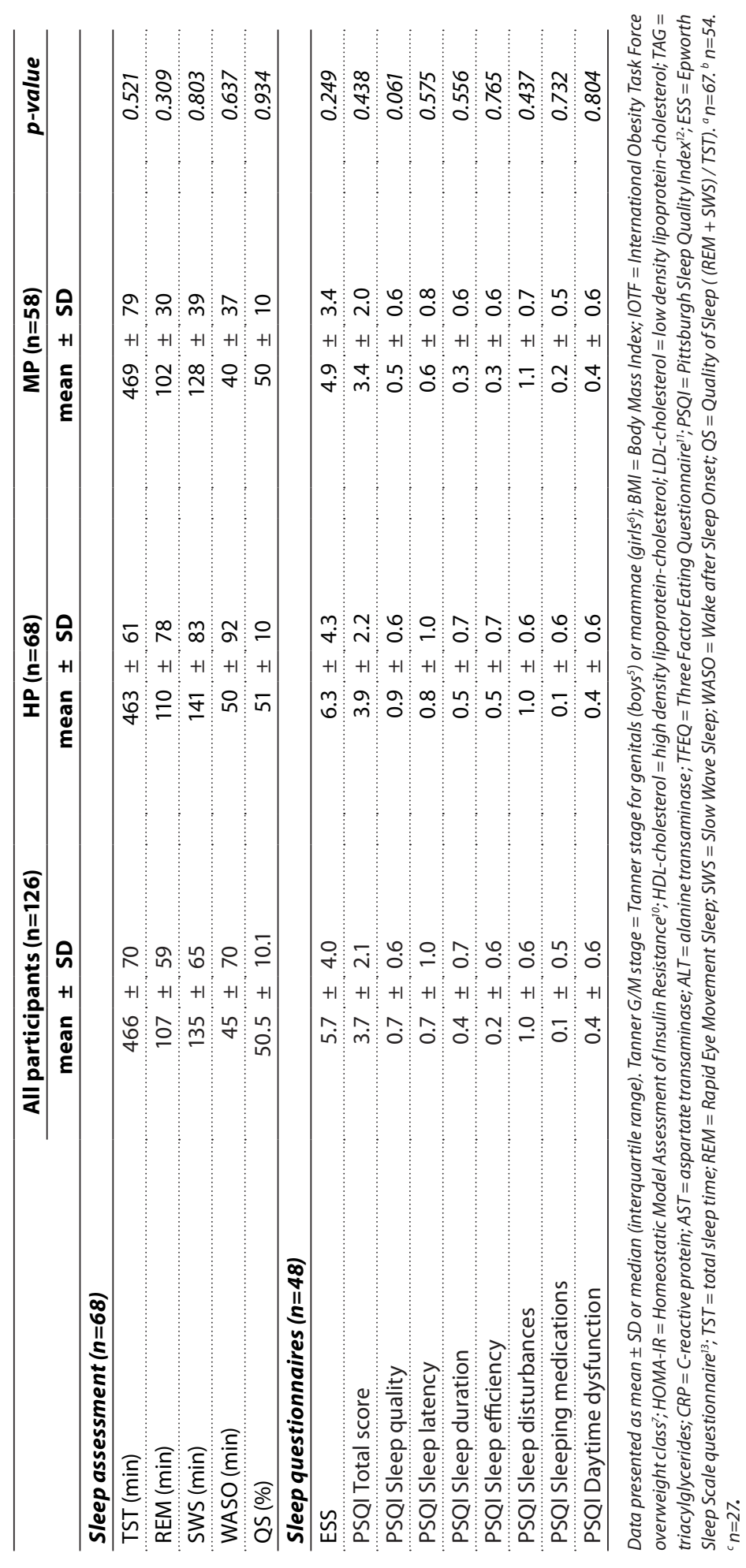




\section{DISCUSSION}

This paper describes the methods and baseline analyses of the PREVIEW study in adolescents. PREVIEW is the first international randomized trial to assess the effects of a high-protein, low-GI and moderate-protein, moderate-GI diet on IR in insulin resistant adolescents, independent of weight changes and puberty. In addition, the longitudinal follow-up (2y) in the PREVIEW study will help fill a gap in the knowledge of HOMA-IR development during puberty in at risk adolescents.

It has been shown that a reduction in BMI is associated with IR reduction in adolescents with overweight and obesity ${ }^{8}$. The DiOGenes study showed that a diet with a higher protein content, especially when combined with a lower Gl, significantly decreased the percentage of overweight and obesity in adolescents of all ages ${ }^{9}$. This study also found that the HP diet improved glucose metabolism even in the absence of BMI z-score changes ${ }^{15}$. Other studies focussing on IR in adolescents often included metformin prescription, which hinders evaluation of the independent effects of diet and physical activity on $\mathrm{IR}^{16}$. Unique about the PREVIEW study is that it studies the impact of a dietary intervention on IR, independent of weight changes, in an at-risk group of insulin resistant adolescents with overweight/obesity during puberty, without providing metformin.

Baseline subject characteristics were not significantly different between the two intervention groups, demonstrating good randomization. No differences were found in HOMA-IR between adolescents in different Tanners stages or between the sexes, which is probably due to including only high-risk adolescents (HOMA-IR $\geq 2.0$ ) in these analyses.

As expected, pubertal adolescents with morbid obesity had a significantly higher HOMA-IR than prepubertal adolescents or adolescents with overweight. High HOMAIR in adolescents with morbid obesity in this and previous studies indicates that these adolescents especially are at high risk for $\beta$-cell exhaustion, which can result in decreased and insufficient $\beta$-cell secretion and T2DM $3,4,17$. The PREVIEW study aims to provide more insight in longitudinal insulin resistance development during puberty in a $2 \mathrm{y}$ follow-up design.

We confirmed that in insulin resistant adolescents with overweight and obesity, parameters of glucose metabolism are associated with increasing puberty stages and markers of adiposity (BMI z-score, FFMI, FMI and FM\%)2. Physical activity, measured as Baecke Sport score, appeared to be inversely associated with fasting blood glucose concentrations, independent of sex, Tanner stage, BMI z-score and FM\%. However, glucose concentrations and accelerometry counts were not related, and Baecke scores were not associated with accelerometry counts. Fasting blood glucose concentrations were positively related to ESS daytime sleepiness.

Strengths of the PREVIEW study are the assessment and monitoring of a wide range of targets associated with reduction of IR, i.e. diet, PA and sleep in adolescents at risk, and 
the international setting. Limitations are the absence of a normal weight control group due to ethical considerations of research in children. Since the research was set in free-living conditions, compliance with the protocol may vary. Response to some lifestyle assessments using questionnaires was quite low due to refusal to answer questionnaires or incomplete questionnaires, limiting interpretation of lifestyle-related outcomes of the intervention. Drop-out analyses revealed some differences between adolescents that did and did not complete all assessments, which will be taken into account in future analyses (Supplementary Table 3.4).

In conclusion, the PREVIEW study is a randomized trial aiming to assess the most effective diet for preventing IR increase, independent of weight change, and corrected for puberty stage, in at risk adolescents with overweight and obesity. Baseline characteristics did not differ between the HP and the MP intervention arms. In adolescents with overweight and obesity at risk for T2DM development, IR was most severe in pubertal adolescents with morbid obesity. IR was associated with sex, adiposity and Tanner stage. Fasting glucose concentrations were independently inversely related to Baecke sport and positively to sleepiness, indicating these might be possible tools for diabetes prevention. 


\section{REFERENCES}

1. Lee JM. Insulin resistance in children and adolescents. Reviews in endocrine \& metabolic disorders. 2006;7(3):141-147.

2. Moran A, Jacobs DR, Jr., Steinberger J, et al. Insulin resistance during puberty: results from clamp studies in 357 children. Diabetes. 1999;48(10):2039-2044.

3. Xu L, Li M, Yin J, et al. Change of Body Composition and Adipokines and Their Relationship with Insulin Resistance across Pubertal Development in Obese and Nonobese Chinese Children: The BCAMS Study. Int J Endocrinol. 2012;2012:389108.

4. Cree-Green M, Triolo TM, Nadeau KJ. Etiology of insulin resistance in youth with type 2 diabetes. Curr Diab Rep. 2013;13(1):81-88.

5. Marshall WA, Tanner JM. Variations in the pattern of pubertal changes in boys. Arch Dis Child. 1970;45(239):13-23.

6. Marshall WA, Tanner JM. Variations in pattern of pubertal changes in girls. Arch Dis Child. 1969;44(235):291-303.

7. Cole TJ, Lobstein T. Extended international (IOTF) body mass index cut-offs for thinness, overweight and obesity. Pediatr Obes. 2012;7(4):284-294.

8. Ho M, Garnett SP, Baur LA, et al. Impact of dietary and exercise interventions on weight change and metabolic outcomes in obese children and adolescents: a systematic review and metaanalysis of randomized trials. JAMA Pediatr. 2013;167(8):759-768.

9. Papadaki A, Linardakis M, Larsen TM, et al. The effect of protein and glycemic index on children's body composition: the DiOGenes randomized study. Pediatrics. 2010;126(5):e1143-1152.

10. Matthews DR, Hosker JP, Rudenski AS, Naylor BA, Treacher DF, Turner RC. Homeostasis model assessment: insulin resistance and beta-cell function from fasting plasma glucose and insulin concentrations in man. Diabetologia. 1985;28(7):412-419.

11. Stunkard AJ, Messick S. The three-factor eating questionnaire to measure dietary restraint, disinhibition and hunger. J Psychosom Res. 1985;29(1):71-83.

12. Buysse DJ, Reynolds CF, 3rd, Monk TH, Berman SR, Kupfer DJ. The Pittsburgh Sleep Quality Index: a new instrument for psychiatric practice and research. Psychiatry Res. 1989;28(2):193213.

13. Johns MW. A new method for measuring daytime sleepiness: the Epworth sleepiness scale. Sleep. 1991;14(6):540-545.

14. Baecke JA, Burema J, Frijters JE. A short questionnaire for the measurement of habitual physical activity in epidemiological studies. Am J Clin Nutr. 1982;36(5):936-942.

15. Damsgaard CT, Papadaki A, Jensen SM, et al. Higher protein diets consumed ad libitum improve cardiovascular risk markers in children of overweight parents from eight European countries. $J$ Nutr. 2013;143(6):810-817.

16. Garnett SP, Gow M, Ho M, et al. Improved insulin sensitivity and body composition, irrespective of macronutrient intake, after a 12 month intervention in adolescents with pre-diabetes; RESIST a randomised control trial. BMC Pediatr. 2014;14:289.

17. Dorenbos E, Rijks JM, Adam TC, Westerterp-Plantenga MS, Vreugdenhil AC. Sleep efficiency as a determinant of insulin sensitivity in overweight and obese adolescents. Diabetes Obes Metab. 2015;17 Suppl 1:90-98.

18. Energy and protein requirements. Report of a joint FAO/WHO/UNU Expert Consultation. World Health Organ Tech Rep Ser. 2013;724:1-206.

19. Fogelholm M, Larsen $T$, Westerterp-Plantenga $M$, et al. PREVIEW: Prevention of Diabetes through Lifestyle Intervention and Population Studies in Europe and around the World. Design, Methods, and Baseline Participant Description of an Adult Cohort Enrolled into a Three-Year Randomised Clinical Trial. Nutrients. 2017;9(6):632.

20. Nena E, Steiropoulos P, Papanas N, et al. Sleepiness as a marker of glucose deregulation in obstructive sleep apnea. Sleep Breath. 2012;16(1):181-186. 


\section{SUPPLEMENTARY DATA FOR MANUSCRIPT}

\section{DETAILED DESCRIPTION OF STUDY PROTOCOL AND METHODS}

\section{Subjects}

\subsection{Inclusion/exclusion criteria}

Inclusion criteria were 1) age 10-17 years, 2) overweight or obesity, defined as age- and sex-adjusted $B M I \geq 25 \mathrm{~kg} / \mathrm{m}^{2} 7$, 3) IR (HOMA-IR $\geq 2.0$ for adolescents Tanner stages $\geq 3$ or any HOMA-IR for adolescents Tanner stages 1-2), 4) written informed consent by both parents and adolescents aged $\geq 12$ years, 5) proficiency of the local language and 6 ) willingness to be randomized and adhere to the study protocol.

Exclusion criteria were 1) medical conditions that might compromise study outcomes or adherence (e.g. T2DM, malabsorption diseases, bariatric surgery, and chronic respiratory, neurological, musculoskeletal disorders), 2) medication use that potentially influenced body weight or glucose metabolism (e.g. metformin) $\leq 3$ months prior to enrolment, 3 ) blood donation or transfusion $\leq 1$ month prior to enrolment, 4) self-reported weight change $\geq 5 \% 2$ months prior to screening, 5) special diets 2 months before screening, 6 ) severe food intolerance, and 7) psychological or behavioural problems leading to difficulty in complying with the protocol.

\subsection{Enrollment}

Adolescents were pre-screened by telephone to assess inclusion and exclusion criteria, and subsequently they underwent a short screening at one of the intervention centres. Adolescents that were found to be eligible at the screening and agreed to continue study participation, were enrolled for baseline measurements and randomization (Figure 1).

\section{Intervention and study protocol}

\subsection{Dietary intervention}

All diets - regardless of intervention group - were aimed at weight stabilization in spite of growth, thus decreasing age- and sex-adjusted BMI z-score. Participants completed a fourday food record at the start of the study, after which a dietician calculated the basal metabolic rate for each child using the WHO formula"1 ${ }^{11}$ All adolescents received personalized sample menus constructed by dieticians, that were in line with their study allocation and energy needs with a maximum of $8700 \mathrm{~kJ} / 24$ hours. During the first study phase, all adolescents received sample menus with the same target macronutrient composition of $15 / 55 / 30 \mathrm{En} \%$ protein/carbohydrate/fat. During the following phase of 96 weeks adolescents received 
personalized sample menus adhering to the targeted macronutrient composition of their randomization arm (Supplementary Table 3.1). In order increase compliance menus were kept as simple as possible and no instructions were given on micronutrient composition and dietary fibre. In addition, adolescents were provided with recipes which were in line with their randomization group and received dietary counselling at each study visit. The consumption of sugar-sweetened beverages and energy-dense foods between meals were discouraged, and the intake of fruits and vegetables stimulated.

\subsection{Physical activity}

Because of natural variability in physical activity in different age categories during childhood, all adolescents received instructions on both high-intensity $(\mathrm{HI})$ and moderate-intensity (MI) PA of which they could choose exercises (Supplementary Table 3.1). Sports in general were encouraged. During each study visit, adolescents were counselled on physical activity.

\section{Measurements}

Measurements were performed during CIDs (Supplementary Table 3.2).

\subsection{Anthropometric measurements and BMI z-score calculation}

Height was measured to $0.1 \mathrm{~cm}$ using a wall-mounted stadiometer (De Grood Metaaltechniek, Nijmegen, the Netherlands) and weight to $0.1 \mathrm{~kg}$ on a digital scale (Seca, Chino, CA, USA). Because mean BMI in childhood is influenced by periods of growth, age- and sex-adjusted $\mathrm{BMI}$ z-scores were calculated to assess BMI deviation in respect to the mean BMI. Since mean BMI has increased during the childhood obesity epidemic, it was decided to calculate BMI z-scores to an older reference cohort as this represented a child's true overweight status. As most of the cohort was Dutch, reference data of the Dutch National Growth Study of 1980 was used to calculate BMI z-scores (Growth Analyser VE, Rotterdam, the Netherlands). Inter-cohort testing showed no difference in height between Dutch, Spanish and British adolescents, making this reference cohort suitable for all adolescents in the study.

\subsection{Body composition}

Body composition was measured using air-displacement plethysmography by the BodPod (Life Measurement Instrument, Concord, CA, USA) or bio-impedance measurements (BIA, Tanita SC-330, Tanita Corp, Tokyo, Japan), after which fat mass (FM), fat free mass (FFM) and fat mass percentage (FM\%) were calculated. Subsequently, fat mass index (FMI) was calculated as fat mass $(\mathrm{kg}) / \mathrm{height}(\mathrm{m})^{2}$, and fat free mass index (FFMI) as fat free mass $(\mathrm{kg}) /$ height $(m)^{2}$.

\subsection{Parameters of glucose metabolism, lipids, inflammation, and liver parameters}

Fasting blood glucose concentrations, total cholesterol, high-density lipoprotein (LDL) 
cholesterol, low-density lipoprotein (HDL) cholesterol, triacylglycerides (TAG), C-reactive protein (CRP), alanine transaminase (ALT), and aspartate transaminase (AST) concentrations were measured with the COBAS 800 modular analyser (Roche, Woerden, the Netherlands). Fasting insulin and $\mathrm{HbA1C}$ concentrations were measured with the fully automated HPLC Variant II 155 (Bio-Rad Laboratories, Veenendaal, the Netherlands) and C-peptide concentration with Immulite XPI (Siemens, Eindhoven, the Netherlands). All laboratory measurements were performed in the Maastricht University Medical Centre laboratory. Insulin sensitivity was assessed by HOMA-IR, a commonly used marker for IR in children because its relatively non-invasive nature (glucose $(\mathrm{mmol} / \mathrm{L}) *$ insulin $(\mathrm{mU} / \mathrm{L}) / 22,5)^{10}$. In the absence of consensus on a HOMA-IR cut-off point for IR, we defined adolescents as insulin resistant when HOMA-IR $\geq 2.0$. Because HOMA-IR is physiologically lower in early pubertal stages while these adolescents still may be at risk of HOMA-IR increase during puberty, all HOMA-IR values were accepted in adolescents at Tanner stages $1-2^{3}$.

\subsection{Blood pressure and heart rate}

Blood pressure and heart rate were measured on the right arm, using the Mobil-OGraph (I.E.M., GmbH, Stolberg, Germany) and a cuff that corresponded with upper arm circumference.

\subsection{Food intake}

Adolescents completed a 4-day food record on paper or through a food diary app to assess food intake and compliance to the study protocol. Food records were analysed at each site for energy intake, macronutrient composition, micronutrients, dietary fibre, Gl and glycaemic load (GL). For the latter, local Gl data for individual food items were used. As a biomarker, $24 \mathrm{~h}$ urinary nitrogen was obtained to calculate protein intake in a subcohort at UM.

\subsection{Physical activity}

PA was measured by 7-day accelerometry (Actigraph GT3X accelerometer, Actigraph Corp, USA). Wear time validation was performed with a minimum of 4 days $>10$ hours including 1 weekend-day.

\subsection{Sleep}

Self-assessed sleep parameters was assessed by the Pittsburgh Sleep Quality Index (PSQI) and Epworth Sleepiness Scale $(E S S)^{12,13}$. In addition, a subcohort at UM underwent a polysomnography to obtain information on total sleeping time (TST), Rapid Eye Movement (REM) sleep, slow wave sleep (SWS), wake after sleep onset (WASO), and quality of sleep (QS, SWS+REM)/TST). 


\section{Primary and secondary endpoints}

The primary endpoint of the PREVIEW study in adolescents was change in HOMA-IR, corrected for puberty, after two years of intervention. Secondary endpoints were changes in $\mathrm{HbA1C}$, BMI Z-score, $\mathrm{FM} \%$, FMI, cardiovascular risk factors, inflammation and liver transaminases, and their associations with HOMA-IR change. Further endpoints included changes in PA and dietary restraint. In a subgroup changes in sleep architecture and their associations with HOMA-IR changes were studied.

\section{Data management}

Data was stored in a central project database at the University of Copenhagen ${ }^{19}$. Anthropometric data was entered in case report forms in the online Open Clinica database. Questionnaires were entered in an Questionnaire Delivery Platform (QDP, NetUnion, Lausanne, Switzerland) or on paper after which the questionnaire was entered in QDP by PREVIEW researchers. Laboratory analyses were centrally performed and entered in a database at UM. Accelerometry data was collected at each site and analysed at SU. Data cleaning was performed by independent researchers at UM and aberrant values checked with a paediatrician.

\section{Statistical analyses}

\subsection{Power calculation}

Considering an estimated $25 \%$ drop-out, a of 0.05 and sample size of 100, a power of 0.96 will be achieved (G*power, Universität Düsseldorf, Düsseldorf, Germany).

\subsection{Analysis for baseline results}

Baseline analyses in this paper were performed using the Statistical Package for the Social Sciences (SPSS) 24.0 (SPSS Inc, IBM Corporation, Armonk, NY, USA). Normal distribution was tested with the Shapiro-Wilk test and outliers were assessed and removed if necessary. ANOVA or Mann-Whitney-U test were used to assess differences in baseline characteristics between the two intervention groups, depending on normality of data. Associations between parameters were assessed with Pearson's or Spearman's correlation coefficients, which were corrected for relevant variables. A $p$-value $<0.05$ was considered statistically significant.

Drop-out analyses, consisting of ANOVA or Mann-Whitney-U tests depending on normality of data, were performed to assess differences in anthropometrics, body composition and glucose metabolism between adolescents that did and did not complete questionnaires. 


\subsection{Future analyses for comparing the two intervention groups}

For comparing the two intervention groups in future analyses, the two dietary arms will be compared using intention-to-treat analyses. Changes over time in HOMA-IR and other outcome measures will be assessed using repeated measurement analyses, and multiple regression analyses will be used to identify the contribution of different variables to HOMAIR change. For comparisons between the two groups, a factorial ANOVA with repeated measures will be used.

\section{Ethical considerations}

Medical Ethics Committees at each study site approved the PREVIEW study protocol and amendments. The study protocol was compliant with the Declaration of Helsinki and the ICH-GCP and registered on ClinicalTrials.gov (number NCT01777893). All study data was handled according to local regulations and the European Directive 95/46/CE. Research staff was GCP trained and UM staff was also trained in clinical paediatrics. Signed informed consent was obtained of parents and adolescents $\geq 12$ years.

\section{DISCUSSION POINTS}

\section{HOMA-IR was significantly higher in pubertal adolescents with morbid obesity compared to prepubertal adolescents with morbid obesity and all adolescents with overweight}

We found that pubertal adolescents with morbid obesity had significantly higher HOMAIR levels than pubertal adolescents with overweight/obesity, identifying this group of adolescents as having a particularly high risk for T2DM development (Supplementary Figure 3.1). This finding confirms earlier studies in which especially adolescents with morbid obesity showed high HOMA-IR at the end of puberty, instead of decreasing HOMA-IR towards the end of puberty as is the pattern in lean adolescents ${ }^{4,17}$. Mechanistically, elevated IR in subjects with morbid obesity might be a direct result of increased ectopic fat storage, which results in increased free fatty acid (FFA) concentrations and inflammation, leading to reduced muscle glucose uptake and thereby maintenance of peripheral IR ${ }^{4}$. High HOMA-IR in late puberty in adolescents with morbid obesity in this and previous studies, demonstrates that these adolescents especially are at high risk for $\beta$-cell exhaustion and T2DM development ${ }^{3,4,17}$.

\section{Fasting blood glucose concentrations were negatively associated with Baecke Sport}

Baecke Sport and fasting blood glucose concentrations were inversely related, independently of sex, Tanner stage, BMI z-score and FM\% (Supplementary Table 3.3). This finding might suggest that higher self-reported PA was associated with better regulated blood glucose concentrations. During exercise, metabolism shifts from predominant reliance on free fatty 
acids (FFA) in rest to carbohydrate oxidation. As glycogen stores in the muscle become deplete, insulin sensitivity of the muscle increases, thereby increasing fasting glucose uptake and muscle insulin sensitivity. Additionally, muscle contractions increase GLUT4 transporter protein translocation and thus enhanced muscle glucose uptake, even in $\mathrm{IR}^{12,13}$. However, glucose metabolism was not associated with accelerometry counts, and Baecke scores and accelerometry data were not interrelated.

\section{Fasting blood glucose concentrations were positively related to sleepiness}

The positive association between fasting blood glucose concentrations and ESS daytime sleepiness scores indicates that adolescents that experienced more sleepiness had higher fasting blood glucose concentrations (Supplementary Table 3.3). This is consistent with an earlier study ${ }^{20}$. Obesity is associated with higher apnoea-hypopnea indexes and intermittent nocturnal hypoxemia, both of which are independently associated with sleepiness and IR. In addition, sleeping time declines during puberty. However, it should be noted that all fasting blood glucose concentrations in this cohort were within normal ranges.

\section{Missing data regarding lifestyle factors}

For some questionnaires and food records, numbers of returned data are relatively low. This is caused by refusal to answer questionnaires, incomplete questionnaires or because questionnaires were not returned. For all questionnaires, a certain number of items have to be filled in to correctly calculate scores, incomplete questionnaires therefore sometimes led to exclusion of the questionnaire for that child for analyses. Food records were often incompletely filled out or not returned at all. Food records $\geq 2$ days of adequate food composition were used for analyses, food records with fewer days or severely inadequately filled out records were excluded for this study.

Drop-out analyses found no differences in adolescents that completed the PA and TFEQ questionnaires and adolescents that did not complete these questionnaires (Supplementary Table 3.4). Adolescents that answered sleep questionnaires had a significantly higher FFMI and fasting glucose concentrations and lower FM\% than adolescents that did not return the sleep questionnaires. These factors will be taken into consideration in future analyses. 


\section{Supplementary tables and figures}

\section{Supplementary table 3.1 Description of the PREVIEW intervention in adolescents}

\begin{tabular}{|c|c|c|}
\hline & HP & MP \\
\hline \multirow[t]{2}{*}{$\begin{array}{l}\text { Dietary } \\
\text { intervention }\end{array}$} & $\begin{array}{l}\text { High protein ( } 25 \text { En\%) } \\
\text { Moderate carbohydrate (45 En\%) } \\
\text { Low GI ( } \leq 50) \text { diet }\end{array}$ & $\begin{array}{l}\text { Moderate protein (15 En\%) } \\
\text { Higher carbohydrate (55 En\%) } \\
\text { moderate GI ( } \geq 56 \text { ) diet }\end{array}$ \\
\hline & $\begin{array}{l}\text { Food items with increased use }{ }^{\text {a: }} \\
\text { - Whole-grain cereals with low } \mathrm{Gl} \\
\text { - Pasta } \\
\text { - Low-fat dairy products } \\
\text { - Poultry } \\
\text { - Fish } \\
\text { - Legumes }\end{array}$ & $\begin{array}{l}\text { Food items with increased use }{ }^{\text {a. }} \\
\text { - Whole-grain cereals with moderate/ } \\
\text { high Gl (e.g. bread) } \\
\text { - Potatoes, sweet potatoes, couscous, rice } \\
\text { - Bananas }\end{array}$ \\
\hline $\begin{array}{l}\text { Physical } \\
\text { activity } \\
\text { intervention }\end{array}$ & \multicolumn{2}{|c|}{$\begin{array}{l}\text { High-intensity physical activity: } \geq 75 \text { minutes per week of high intensity physical } \\
\text { activity, such as vigorous bicycling, jogging }>8 \mathrm{~km} / \mathrm{h} \text { and strenuous ball games and } \\
\text { moderate-intensity physical activity: } \geq 150 \text { minutes per week of moderate intensity } \\
\text { activity, such as moderate bicycling, brisk walking }(4-6 \mathrm{~km} / \mathrm{h}) \text {, and swimming }\end{array}$} \\
\hline
\end{tabular}

$G I=$ Glycaemic Index $; H P=$ high-protein low-GI diet; $M P=$ moderate-protein moderate-GI diet En $\%$ = Energy percentage .

a. Increased use relative to the other intervention group

${ }^{b}$ Both groups received instructions for both PA intensities 
Supplementary table 3.2: Overview of data collection at different Clinical Investigation Days (CID) in the PREVIEW adolescents intervention

\begin{tabular}{|c|c|c|c|c|c|c|}
\hline \multirow[t]{2}{*}{ Data collection } & \multicolumn{6}{|c|}{ Assessment time-points (week) } \\
\hline & $\begin{array}{c}0 \\
\text { CID1 }\end{array}$ & $\begin{array}{c}8 \\
\text { CID2 }\end{array}$ & $\begin{array}{c}26 \\
\text { CID3 }\end{array}$ & $\begin{array}{c}52 \\
\text { CID4 }\end{array}$ & $\begin{array}{c}78 \\
\text { CID5 }\end{array}$ & $\begin{array}{l}104 \\
\text { CID6 }\end{array}$ \\
\hline Randomization & $\mathrm{X}$ & & & & & \\
\hline $\begin{array}{l}\text { General information } \\
\text { Age (y) } \\
\text { Tanner G/M stage }\end{array}$ & $x$ & $\mathrm{X}$ & $\mathrm{X}$ & $x$ & $x$ & $x$ \\
\hline $\begin{array}{l}\text { Anthropometric characteristics } \\
\text { - Body weight (kg) } \\
\text { - Height (cm) } \\
\text { - Sitting height (subgroup) } \\
\text { - BMI (kg/m2) } \\
\text { - BMI z-score (SD) } \\
\text { - } \text { OTF class } \\
\text { - Waist circumference }(\mathrm{cm}) \\
\text { - Hip circumference }(\mathrm{cm}) \\
\text { - Thigh circumference }(\mathrm{cm})\end{array}$ & $\mathrm{x}$ & $\mathrm{X}$ & $\mathrm{X}$ & $x$ & $x$ & $x$ \\
\hline $\begin{array}{l}\text { Body composition } \\
\text { - Fat free mass index (FFMl, kg/m2) } \\
\text { - Fat mass index (FMl, kg/m2) } \\
\text { - Fat mass (\%) }\end{array}$ & $x$ & $\mathrm{X}$ & $\mathrm{X}$ & $x$ & $x$ & $x$ \\
\hline $\begin{array}{l}\text { Parameters of glucose metabolism } \\
\text { - Fasting glucose, fasting insulin, HOMA-IR, HbA1c, } \\
\text { C-peptide }\end{array}$ & $x$ & $\mathrm{X}$ & $x$ & $x$ & $x$ & $x$ \\
\hline $\begin{array}{l}\text { Lipids } \\
\text { - Total cholesterol, HDL-cholesterol, LDL-cholesterol, TAG }\end{array}$ & $x$ & $x$ & $x$ & $x$ & $x$ & $x$ \\
\hline $\begin{array}{l}\text { Inflammation } \\
\text {. CRP }\end{array}$ & $x$ & $\mathrm{X}$ & $x$ & $x$ & $x$ & $x$ \\
\hline $\begin{array}{l}\text { Liver parameters } \\
\text { - AST, ALT }\end{array}$ & $\mathrm{X}$ & $\mathrm{X}$ & $x$ & $x$ & $x$ & $x$ \\
\hline $\begin{array}{l}\text { Blood pressure and heart rate } \\
\text { - Systolic and diastolic blood pressure } \\
\text { - Heart rate }\end{array}$ & $x$ & $\mathrm{X}$ & $\mathrm{X}$ & $x$ & $x$ & $x$ \\
\hline $\begin{array}{l}\text { Physical activity } \\
\text { - } 7 \text {-day accelerometry } \\
\text { - Baecke questionnaire }\end{array}$ & $\mathrm{x}$ & $\mathrm{X}$ & $\mathrm{X}$ & $\mathrm{X}$ & & $\mathrm{X}$ \\
\hline $\begin{array}{l}\text { Food intake behaviour } \\
\text { - 4-day food record } \\
\text { - TFEQ questionnaire } \\
\text { - VAS appetite scores }\end{array}$ & $\mathrm{X}$ & & $\mathrm{X}$ & $X$ & & $x$ \\
\hline $\begin{array}{l}\text { Protein intake } \\
\text {. Urinary nitrogen (subgroup) } \\
\end{array}$ & $x$ & & & $X$ & & $X$ \\
\hline $\begin{array}{l}\text { Sleep assessment } \\
\text { - Polysomnography (subgroup) }\end{array}$ & $x$ & & & $X$ & & $x$ \\
\hline $\begin{array}{l}\text { Sleep questionnaires } \\
\text { - PSQI sleep questionnaire } \\
\text { - ESS Sleep questionnaire }\end{array}$ & $x$ & $\mathrm{X}$ & $\mathrm{X}$ & $x$ & & $x$ \\
\hline
\end{tabular}

$C I D=$ Clinical Investigation Day; Tanner G/M stage = Tanner stage for genitals $\left(\right.$ boys $\left.^{8}\right)$ or mammae $\left(\right.$ girls $\left.s^{9}\right) ; B M I=$ Body Mass Index; IOTF = International Obesity Task Force overweight class'; HOMA-IR = Homeostatic Model Assessment of Insulin Resistance $;$; HDLcholesterol = high density lipoprotein-cholesterol; $L D L$-cholesterol = low density lipoprotein-cholesterol; TAG = triacylglycerides; $C R P=c$-reactive protein; $A S T=$ aspartate transaminase; $A L T=$ alanine transaminase; $T F E Q=$ Three Factor Eating Questionnaire ${ }^{10}$; $P S Q I=$ Pittsburgh Sleep Quality Index ${ }^{4} ; E S S=$ Epworth Sleep Scale questionnaire ${ }^{5}$. 
Supplementary table 3.3: Correlation coefficients for physical activity, food intake behaviour, and sleep with parameters of glucose metabolism, corrected for sex, Tanner stage, BMI z-score, and $\mathrm{FM} \%$

\begin{tabular}{|c|c|c|c|c|c|c|}
\hline & & $\begin{array}{r}\text { Glucose } \\
(\mathrm{mmol} / \mathrm{L})\end{array}$ & $\begin{array}{r}\text { Insulin } \\
(\mathrm{pmol} / \mathrm{L})\end{array}$ & HOMA-IR & $\begin{array}{r}\text { HbA1c } \\
(\mathrm{mmol} / \mathrm{l})\end{array}$ & $\begin{array}{r}\text { C-peptide } \\
(\mathrm{nmol} / \mathrm{L})\end{array}$ \\
\hline \multicolumn{7}{|l|}{ Physical activity } \\
\hline Baecke Work & $r$ & 0.190 & 0.069 & 0.114 & 0.242 & 0.094 \\
\hline Baecke Sport & $r$ & $-0.223^{*}$ & -0.105 & -0.157 & -0.142 & -0.140 \\
\hline Baecke Leisure & $r$ & -0.118 & -0.028 & 0.173 & 0.032 & -0.046 \\
\hline Baecke total score & $r$ & -0.096 & 0.059 & 0.073 & 0.068 & -0.139 \\
\hline Counts (cpm) & $r$ & -0.068 & -0.088 & -0.083 & 0.231 & 0.157 \\
\hline \multicolumn{7}{|l|}{ Food intake behaviour } \\
\hline $\begin{array}{l}\text { TFEQ cognitive restraint } \\
\text { of hunger }\end{array}$ & $r$ & -0.164 & 0.018 & -0.067 & 0.010 & -0.105 \\
\hline TFEQ disinhibition & $r$ & 0.072 & -0.112 & -0.071 & 0.019 & -0.150 \\
\hline TFEQ hunger & $r$ & -0.039 & 0.024 & 0.015 & 0.149 & -0.037 \\
\hline \multicolumn{7}{|l|}{ Sleep questionnaires } \\
\hline PSQI & $r$ & -0.162 & -0.216 & -0.209 & -0.361 & -0.202 \\
\hline ESS & $r$ & $0.280^{*}$ & -0.002 & 0.020 & 0.258 & 0.041 \\
\hline \multicolumn{7}{|l|}{ Sleep assessment } \\
\hline TST (min) & $r$ & $-0,065$ & 0,082 & 0,115 & $-0,02$ & $-0,063$ \\
\hline SWS (min) & $r$ & $-0,093$ & $-0,004$ & $-0,026$ & $-0,089$ & $-0,050$ \\
\hline REM (min) & $r$ & $-0,171$ & $-0,031$ & $-0,047$ & $-0,002$ & $-0,171$ \\
\hline SE (\%) & $r$ & $-0,039$ & 0,047 & 0,103 & 0,037 & 0,042 \\
\hline QS (\%) & $r$ & $-0,163$ & 0,046 & $-0,014$ & $-0,075$ & $-0,024$ \\
\hline WASO (min) & $r$ & 0,034 & $-0,139$ & $-0,163$ & $-0,164$ & $-0,179$ \\
\hline
\end{tabular}

HOMA-IR = Homeostatic Model Assessment of Insulin Resistance ${ }^{10} ;$ TFEQ = Three Factor Eating Questionnaire ${ }^{11} ;$ PSQI = Pittsburgh Sleep Quality Index ${ }^{12} ;$ ESS = Epworth Sleep Scale questionnaire ${ }^{13} ;$ TST = total sleep time; REM = Rapid Eye Movement Sleep; SWS = Slow Wave Sleep; WASO = Wake after Sleep Onset; $Q S=$ Quality of Sleep $((R E M+S W S) / T S T) .{ }^{*} p<0.05$ 


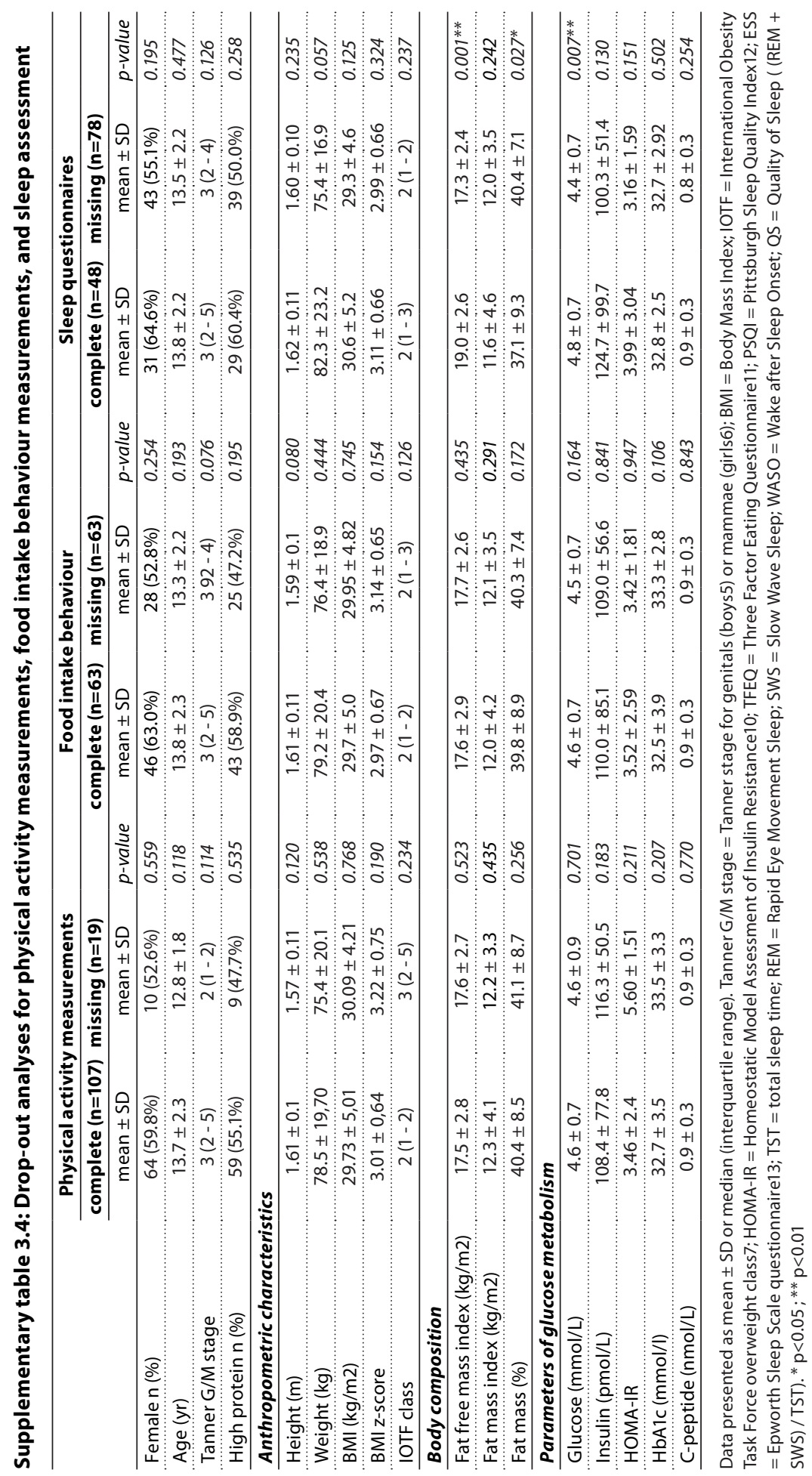




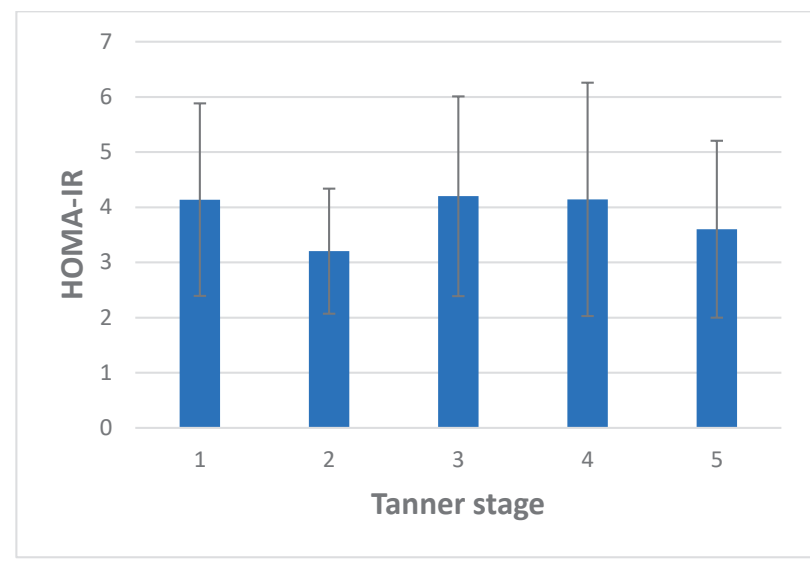

A

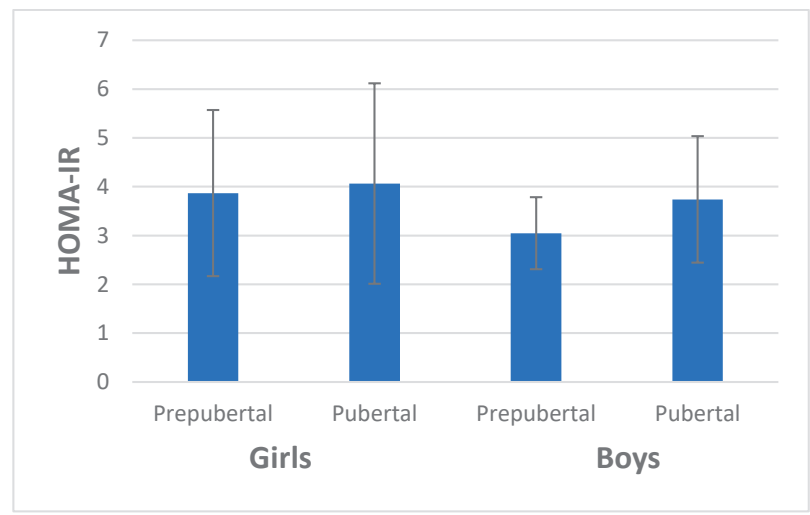

B

\section{C}

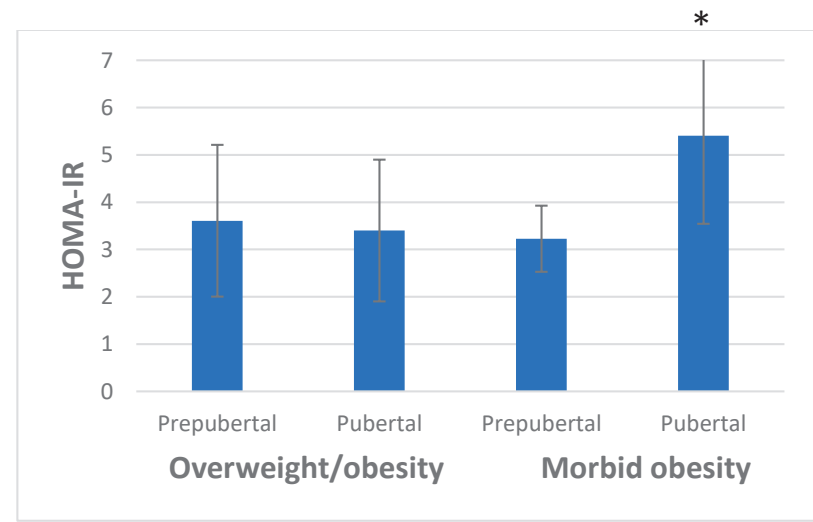

\section{Supplementary figure 3.3: HOMA-IR for different puberty stages}

$H O M A-I R$ at different puberty stages in adolescents with HOMA-IR $\geq 2.0(n=94)$, presented as mean $\pm S D$.

A) HOMA-IR in adolescents in different Tanner stages. Mean HOMA-IR was not different between adolescents in the different puberty stages. B) HOMA-IR in prepubertal and pubertal boys and girls. No differences in HOMAIR were found between the groups. C) HOMA-IR in prepubertal and pubertal adolescents with overweight/ obesity and morbid obesity. Pubertal adolescents with morbid obesity had significant higher mean HOMA$I R$ compared to the adolescents in the other groups. HOMA-IR = Homeostatic Model Assessment of Insulin Resistance ${ }^{10}$. Prepubertal: Tanner G/M stage 1-2. Pubertal: Tanner G/M stage 3-5. * $p<0.01$. 


\section{REFERENCES}

1. Cole TJ, Lobstein T. Extended international (IOTF) body mass index cut-offs for thinness, overweight and obesity. Pediatric obesity. 2012;7(4):284-294.

2. Matthews DR, Hosker JP, Rudenski AS, Naylor BA, Treacher DF, Turner RC. Homeostasis model assessment: insulin resistance and beta-cell function from fasting plasma glucose and insulin concentrations in man. Diabetologia. 1985;28(7):412-419.

3. Xu L, Li M, Yin J, et al. Change of Body Composition and Adipokines and Their Relationship with Insulin Resistance across Pubertal Development in Obese and Nonobese Chinese Children: The BCAMS Study. International journal of endocrinology. 2012;2012:389108.

4. Buysse DJ, Reynolds CF, 3rd, Monk TH, Berman SR, Kupfer DJ. The Pittsburgh Sleep Quality Index: a new instrument for psychiatric practice and research. Psychiatry research. 1989;28(2):193-213.

5. Johns MW. A new method for measuring daytime sleepiness: the Epworth sleepiness scale. Sleep. 1991;14(6):540-545.

6. Cree-Green M, Triolo TM, Nadeau KJ. Etiology of insulin resistance in youth with type 2 diabetes. Current diabetes reports. 2013;13(1):81-88.

7. Dorenbos E, Rijks JM, Adam TC, Westerterp-Plantenga MS, Vreugdenhil AC. Sleep efficiency as a determinant of insulin sensitivity in overweight and obese adolescents. Diabetes, obesity \& metabolism. 2015;17 Suppl 1:90-98.

8. Marshall WA, Tanner JM. Variations in the pattern of pubertal changes in boys. Archives of disease in childhood. 1970;45(239):13-23.

9. Marshall WA, Tanner JM. Variations in pattern of pubertal changes in girls. Archives of disease in childhood. 1969;44(235):291-303.

10. Stunkard AJ, Messick S. The three-factor eating questionnaire to measure dietary restraint, disinhibition and hunger. Journal of psychosomatic research. 1985;29(1):71-83.

11. Energy and protein requirements. Report of a joint FAO/WHO/UNU Expert Consultation. World Health Organization technical report series. 2013;724:1-206.

12. Fogelholm M, Larsen $T$, Westerterp-Plantenga $M$, et al. PREVIEW: Prevention of Diabetes through Lifestyle Intervention and Population Studies in Europe and around the World. Design, Methods, and Baseline Participant Description of an Adult Cohort Enrolled into a Three-Year Randomised Clinical Trial. Nutrients. 2017;9(6):632.

13. Nena E, Steiropoulos $\mathrm{P}$, Papanas $\mathrm{N}$, et al. Sleepiness as a marker of glucose deregulation in obstructive sleep apnea. Sleep \& breathing. 2012;16(1):181-186. 


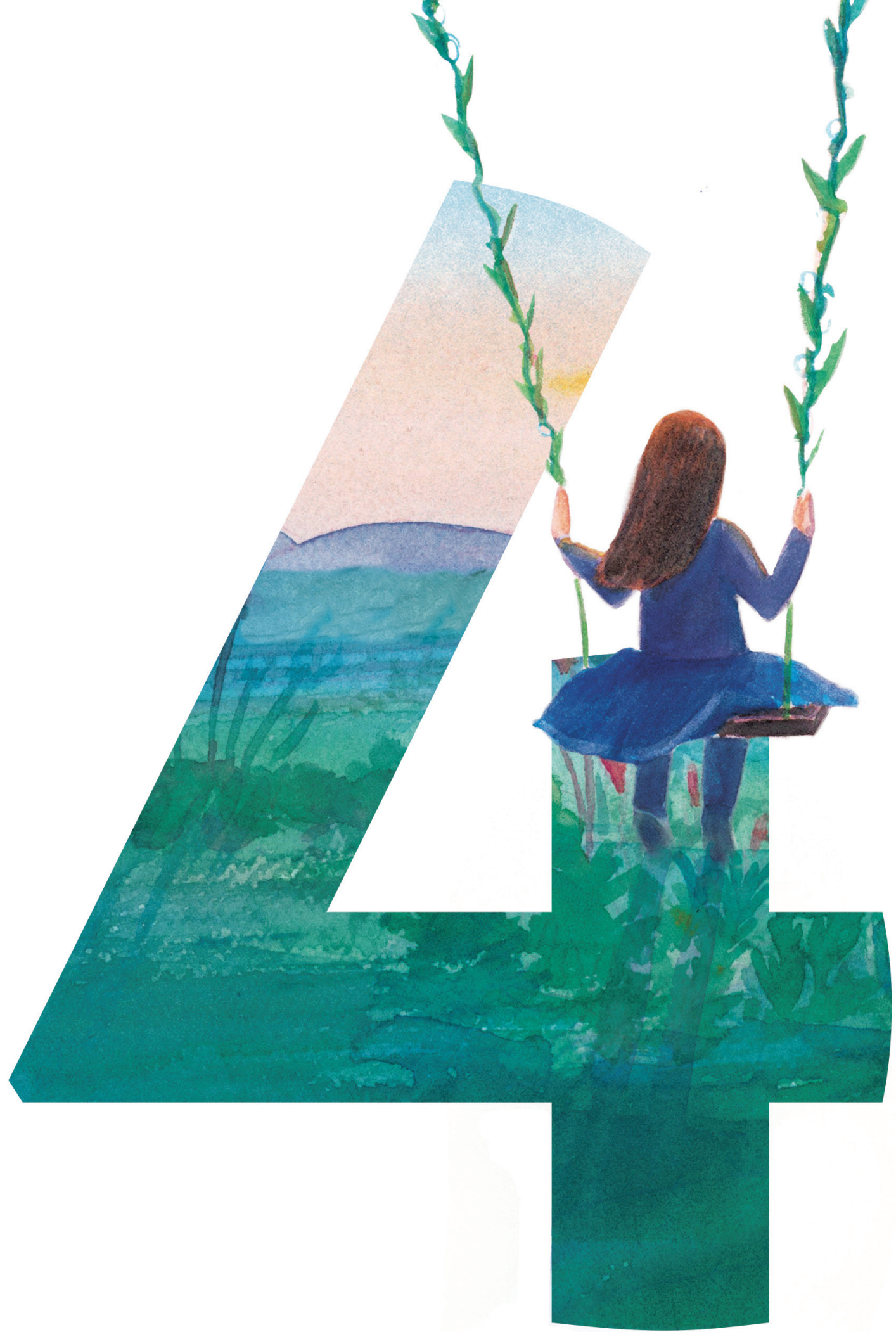




\section{Chapter 4}

\section{Effect of a high-protein low-Gl diet on insulin resistance in adolescents with overweight/obesity \\ - a PREVIEW Randomized Controlled Trial}

\author{
Elke Dorenbos \\ Mathijs Drummen \\ Tanja Adam \\ Jesse Rijks \\ J. Alfredo Martínez \\ Santiago Navas-Carretero \\ Gareth Stratton \\ Nils Swindell
}

\author{
Pauline Stouthart \\ Kelly Mackintosh \\ Melitta Mcnarry \\ Angelo Tremblay \\ Mikael Fogelholm \\ Anne Raben \\ Margriet Westerterp-Plantenga \\ Anita Vreugdenhil
}




\section{ABSTRACT}

\section{Background}

Pubertal insulin resistance (IR) is associated with increased risk of type 2 diabetes mellitus development in adolescents with overweight/obesity.

\section{Background}

The PREVIEW study was a randomized parallel trial assessing the effect of increasing dietary protein intake on IR in adolescents with overweight/obesity. It was hypothesized that increase of protein intake would result in a relatively lower IR.

\section{Methods}

Adolescents with overweight/obesity and IR from the Netherlands, UK and Spain were randomized into a moderate-protein-moderate-GI (15/55/30En\% protein/carbohydrate/ fat, $G l \geq 56)$ or high-protein-low-Gl (25/45/30En\% protein/carbohydrate/fat, $G \mid<50)$ diet. Anthropometric and cardiometabolic parameters, puberty, dietary intake and physical activity (PA) were measured and related to changes in BMI Z-score and HOMA-IR.

\section{Results}

126 adolescents were included in this study $(13.6 \pm 2.2 y$, BMI z-score $3.04 \pm 0.66$, HOMA-IR $3.48 \pm 2.28, \mathrm{HP} n=68, \mathrm{MP} n=58$ ). Changes in protein intake were not significantly different between timepoints or intervention groups and no effects of the intervention on IR were observed. Post-hoc, BMI z-score decreased after 1y $(-0.16(95 \% \mathrm{Cl}-0.24,-0.07), \mathrm{p}<0.001)$ and $2 y(-0.19(-0.36,-0.02), p=0.028)$. HOMA-IR was positively associated with BMI z-score change $(B=2.23(2.07 ; 2.40), p<0.001)$. Cognitive restraint was increased $(2.1(0.7,3.5), p=0.003) ; B M I$ $z$-score change was inversely related to dietary restraint change $(B=-0.03(-0.05,-0.01)$, $p=0.045)$, and positively to susceptibility to hunger $(B=0.03(0.01 ; 0.06), p=0.013)$.

\section{Conclusions}

The PREVIEW study observed no effect of a high-protein low-GI diet on insulin resistance in adolescents with overweight/obesity and insulin resistance because of lack of feasibility. Post-hoc, stabilization pubertal insulin resistance was associated with BMI z-score decrease. BMI z-score decrease was associated with increased dietary restraint. 


\section{INTRODUCTION}

The prevalence of childhood obesity has rapidly increased over the last decades and, without adequate management, is expected to rise to approximately 268 million children globally in 2025 with a subsequent increase in obesity-related comorbidities ${ }^{1-3}$. During puberty, transient insulin resistance (IR) is a common physiological phenomenon ${ }^{4-9}$. IR is defined as reduced ability of insulin to increase glucose uptake and utilization, resulting in a compensatory increase in insulin secretion to maintain normal blood glucose concentrations ${ }^{7}$. A transient increase in IR during puberty is considered to be a physiological phenomenon of growth, but especially adolescents with obesity show an exaggerated increase in IR. In addition, in adolescents with obesity, IR does not appear to decrease at the end of puberty as is observed in lean adolescents ${ }^{5,6,9}$. Particularly adolescents with obesity therefore may have increased risk for $\beta$-cell exhaustion and development of T2DM even at a young age ${ }^{5}$. Furthermore, it has been shown that both obesity and IR are associated with development of cardiovascular disease (CVD) e.g. dyslipidaemia and hypertension, and non-alcoholic fatty liver disease (NAFLD) even in childhood ${ }^{8,10}$. Moreover, insulin resistant adolescents were less successful in decreasing BMI z-score in response to interventions than adolescents that were not insulin resistant ${ }^{4,11}$. Thus, particularly in adolescents with overweight or obesity and increased IR, interventions should focus on decreasing BMI z-score and assess whether the increase of HOMA-IR during pubertal IR can be attenuated.

Recommendations of the US Preventive Services Task Force and others include comprehensive, multidisciplinary lifestyle interventions for treatment of obesity in children, although there is no consensus on the most efficient and effective type of dietary and physical activity strategy ${ }^{11,12}$. Earlier observations reported that a relative increase of dietary protein, thereby reducing fat and carbohydrate intake, led to a significant reduction in obesity $^{11-18}$. Two studies showed that a higher-protein diet reduced IR significantly ${ }^{12,17}$. One study that combined increased protein intake with decreased glycaemic index (GI) observed a significant reduction in percentage of children with overweight/obesity in this group compared to control diets ${ }^{17}$. Proposed underlying mechanisms for these effects might be the ability of protein to increase satiety, thermogenesis and fat-oxidation, thus reducing fat mass while maintaining fat free mass during energy restriction ${ }^{19}$. Lowering $\mathrm{Gl}$ in diets has been suggested to promote satiety and reduces hunger, although few longterm studies have been performed in adolescents to confirm these effects ${ }^{20}$. In addition, physical activity (PA) alone and in combination with a dietary intervention, has been shown to change body composition by increasing fat free mass, and reducing fat mass, fasting glucose concentrations and $\mathrm{IR}^{21-23}$. Especially lifestyle interventions combining diet, PA, and behavioural strategies have been effective in decreasing obesity and reducing IR and cardiovascular risk parameters in youths ${ }^{22}$. Studies researching the effects of combined lifestyle intervention in adolescents with overweight/obesity and increased IR are scarce. 
Thus far three large community studies have been performed aiming to decrease insulin resistance parameters in adolescents with overweight/obesity, of which two showed significant favourable changes in glucose metabolism abnormalities and BMI z-score after short-term, intensive intervention ${ }^{24-26}$. Lifestyle interventions in adolescents in freeliving conditions often report problems with participants meeting dietary targets and maintaining physical activity (PA) levels ${ }^{26,27}$. Specifically studies aiming to increase relative protein intake reported difficulties in dietary compliance and participants meeting protein targets, and were mostly conducted under controlled settings (e.g. meal observation in in-centre settings) $)^{11,13-18}$. Therefore, the effect of a lifestyle intervention combining a high protein intake, low GI diet in adolescents with overweight/obesity and increased IR, should be addressed in real-life settings.

The PREVention of diabetes through lifestyle Intervention and population studies in Europe and around the World (PREVIEW) study in adolescents was a randomized clinical study of the effect of a high-protein low-GI diet in adolescents with overweight or obesity and on IR. It was hypothesized that a high-protein low-GI diet would be superior in reducing IR compared to a medium-protein medium-GI diet, in insulin resistant adolescents with overweight or obesity.

\section{MATERIALS AND METHODS}

\section{Study design}

The PREVIEW study in adolescents was a multicentre, 104-week parallel-group, genderstratified block-randomized (10:10) trial between December 2013 and December 2018, as described previously ${ }^{28}$. The first eight weeks aimed at weight stabilization during growth. All participants received sample menus based on their estimated energy requirements, consisting of 15/55/30 energy percent (En\%) protein/carbohydrate/fat ${ }^{29}$. In the second phase adolescents were randomized into a moderate-protein moderate-GI (MP) group or a high-protein low-Gl (HP). Randomization was stratified by sex, age and centre in blocks of 10 using a computerized randomization tool. The MP group received a sample menu with a macronutrient composition of 15/55/30 En\% protein/carbohydrate/fat and a Gl $\geq 56$. The HP group received a sample menu with a target macronutrient composition of 25/45/30 En\% protein/carbohydrate/fat and a $\mathrm{Gl} \leq 50$. All menus were tailored to the participant's estimated energy requirements. Upon request, further personalized tips were given taking e.g. cultural traditions into account. Participants were instructed to increase PA (in organized sports and daily movement) and received booklets with exercises for high and medium intensity PA. Due to the personalized instructions for participants during the measurement meetings, participants and research staff could not be blinded. The study was designed as a $2 y$ randomized clinical trial. The study protocol was approved by local Medical Ethics 
Committees at all study sites. The study was compliant with the Declaration of Helsinki and ICH-GCP and published on ClinicalTrials.gov (no. NCT01777893).

\section{Participants}

Adolescents were recruited from three study sites (Maastricht University, the Netherlands; University of Navarra, Spain and Swansea University, United Kingdom) between December 2013 and December 2016 by ED, NS and SNC. Inclusion criteria were overweight/obesity (BMI z-score >1.0 SDS), increased IR (defined as Homeostatic Model Assessment of Insulin Resistance (HOMA-IR) > 2.0 for adolescents at Tanner G/M stages $\geq 3$ or any HOMA-IR for adolescents at Tanner stages 1-2) and signed informed consent from both parents and adolescents $\geq 12$ years ${ }^{28}$. Exclusion criteria included medical conditions or use of medication that might influence study outcomes (e.g. T2DM, bariatric surgery, and use of metformin) or compromise study adherence (e.g. severe food intolerances or musculoskeletal diseases).

\section{Measurements}

\subsection{Anthropometric characteristics and body composition}

Height and weight were measured at baseline, after 1 and $2 y$ while participants were barefoot, wearing only underwear and in a fasted state, and subsequently BMI was calculated. Because BMI in adolescents is not a representative measure of obesity status due to periods of growth, age- and gender-adjusted BMI z-scores were calculated (TNO Growth Calculator, TNO, Den Haag, the Netherlands) ${ }^{30}$. Body composition was measured with airdisplacement plethysmography (at Maastricht University: BodPod, Life Measurement Instruments, Concord, CA, USA) using the Lohman algorithm, bio-impedance measurements (at University of Navarra: BIA, Tanita SC-330. Tanita Corp, Tokyo, Japan), or dual energy x-ray absorptiometry (at Swansea University: DEXA, Stratos dR, Medimaging UK) ${ }^{31}$. Pubertal stage was determined with the Tanner genital (boys) or mammary (girls) scale ${ }^{32,33}$.

\subsection{Glucose metabolism, lipid spectrum, inflammation, liver parameters and blood pressure} Blood samples were obtained by venepuncture after an overnight fast by trained healthcare professionals. All samples were centrifuged and frozen locally, and subsequently analysed at the laboratory for clinical chemistry at Maastricht University. Concentrations of fasting blood glucose, total cholesterol, high-density lipoprotein (HDL) cholesterol, lowdensity lipoprotein (LDL) cholesterol, triglycerides (TAG), C-reactive protein (CRP), alanine transaminase (ALT), and aspartate transaminase (AST) were analysed with the COBAS 800 modular analyser (Roche, Woerden, the Netherlands). Fasting insulin and HbA1c concentrations were determined using the fully automated HPLC Variant II 155 (Bio-Rad Laboratories, Veenendaal, the Netherlands) and C-peptide concentrations with the Immulite XPI (Siemens, Eindhoven, the Netherlands). An index for insulin resistance was calculated 
using HOMA-IR (fasting glucose concentration ( $\mathrm{mmol} / \mathrm{L}$ ) * fasting insulin concentration $(\mathrm{m} \mathrm{U} / \mathrm{L}) /$ $22.5)^{34}$. Blood pressure and heart rate were measured while seated, on the right upper arm using a matching cuff size (Mobil-O-Graph, I.E.M., GmbH, Stolberg, Germany or Omron M6 comfort HEM-7221-E8, Omron Corp., Kyoto, Japan).

\subsection{Compliance: food intake and physical activity}

Compliance to the dietary instructions was assessed using four-day food records, provided at each measurement visit, and which were subsequently analysed for energy intake, macronutrient composition including protein content (both $\mathrm{g} / \mathrm{d}$ as En\%), fibre content, Gl and glycaemic load (GL) using the Eetmeter food diary \& analysis tool (Voedingscentrum, Den Haag, the Netherlands). In addition, participants answered the Three Factor Eating Questionnaire(TFEQ), consisting of the three factors cognitive restraint of eating, disinhibition and hunger ${ }^{35}$. To assess underreporting, reported total energy intake was compared to the daily energy requirements for children and adolescents using the WHO formula to assess underreporting (total energy expenditure (MJ/day) $=1.298+0.265 \mathrm{~kg}-0.0011 \mathrm{~kg}^{2}$ (boys) or $\left.1.102+0.273 \mathrm{~kg}-0.0019 \mathrm{~kg}^{2}(\mathrm{gir} / \mathrm{s})\right)^{29}$. PA was assessed with 7-day accelerometry (Actigraph GT3X accelerometer, Actigraph Corp, USA) and the Baecke Questionnaire ${ }^{36}$. Participants were instructed to wear the accelerometer on the right hip during 7 full days and nights, only removing the accelerometer during showering, swimming or contact sports. Wear time validation was performed with a minimum of 4 days $>10$ hours including 1 weekendday. Epochs were measured with the length of 10 seconds and Evenson cut-off points were used to assess moderate, light and vigorous activity ${ }^{37,38}$.

\section{Statistical analyses}

Power calculations were performed using $G^{*}$ power (Dusseldorf University, Dusseldorf, Germany) and adjusted for an estimated $25 \%$ drop-out. With an a of 0.05 , effect size of 0.37 for HOMA-IR and sample size of 100 , a power of 0.96 could be achieved $^{28}$. To remain sufficient power for analyses at 1 and $2 y$ despite drop-out, intention-to-treat analyses were performed on the complete dataset after multiple imputation. For this 50 datasets were created (MICE Package in R, v3.2.3, Vienna, Austria). The maximum number of iterations was set to 20, where convergence was checked by inspecting the trace lines. The following predictors were used to impute missing values: gender, age, Tanner stage and BMI z-score at baseline and at 1 or $2 y$, and the baseline value of the imputed variable. For blood pressure, height at baseline and at 1 or $2 y$ were added as predictors. Estimated effect changes over time in the intention-to-treat analyses were pooled from the multiple imputed datasets, analysed using factorial ANOVA's with repeated measures and presented as mean (95\% confidence interval). As this was an exploratory study, no corrections for multiple comparisons have been made. 
Measured data from the adolescents who stayed in the study were analysed for changes over time, using ANOVA repeated measures. All statistical analyses were performed using IBM SPSS Statistics for Windows version 24 (IBM Corp., Armonk, NY, USA). For differences between the two intervention groups at baseline and comparing those who dropped out to completers, T-tests and factorial ANOVA and Mann-Whitney-U test were used. Factorial ANOVA's with repeated measures were used for assessing differences over time in the completers group. Post-hoc associations for dietary and PA variables with changes in BMI $z$-score were performed using multiple linear regression analyses, with change in Tanner stage as a covariate. Post hoc associations for dietary and PA variables with HOMA-IR were performed with multiple linear regression analyses, with change in BMI z-score and Tanner stage as covariates. A two-sided p-value smaller than 0.05 was considered statistically significant.

\section{RESULTS}

\section{Characteristics of participants}

In total, 126 adolescents were included in the baseline analyses between December 2013 and December 2016, as described previously ${ }^{26}$. After 1y of intervention 83 adolescents (66\%) were still participating, and after 2y 49 participants (39\%) completed the study (Figure 4.1). Reasons for drop-out were discontinuation of the study due to personal reasons $(n=4)$ and loss to follow-up ( $n=77$ ). Baseline characteristics of adolescents who dropped out were not significantly different from adolescents that remained in the study. No serious adverse events (SAE) were reported.

Baseline descriptives of the HP and MP group are presented in Tables 4.1A+B. No significant differences between the groups were observed in gender, BMI z-score, HOMA-IR or other cardiometabolic or lifestyle parameters. Baseline descriptives of the adolescents that completed analyses at $1 \mathrm{y}$ and $2 \mathrm{y}$ are presented in Tables 4.2A+B. Here, too, no significant differences between the groups were observed in baseline demographics, cardiovascular or lifestyle variables.

\section{Compliance to dietary instructions}

Absolute and relative reported protein intake, as a percentage of total energy intake, were not significantly changed after $1 \mathrm{y}$ and $2 \mathrm{y}$ of intervention in the HP and MP group (Tables $4.1 A \& 4.2 A)$. No significant differences were observed between the two intervention groups regarding dietary intake. 


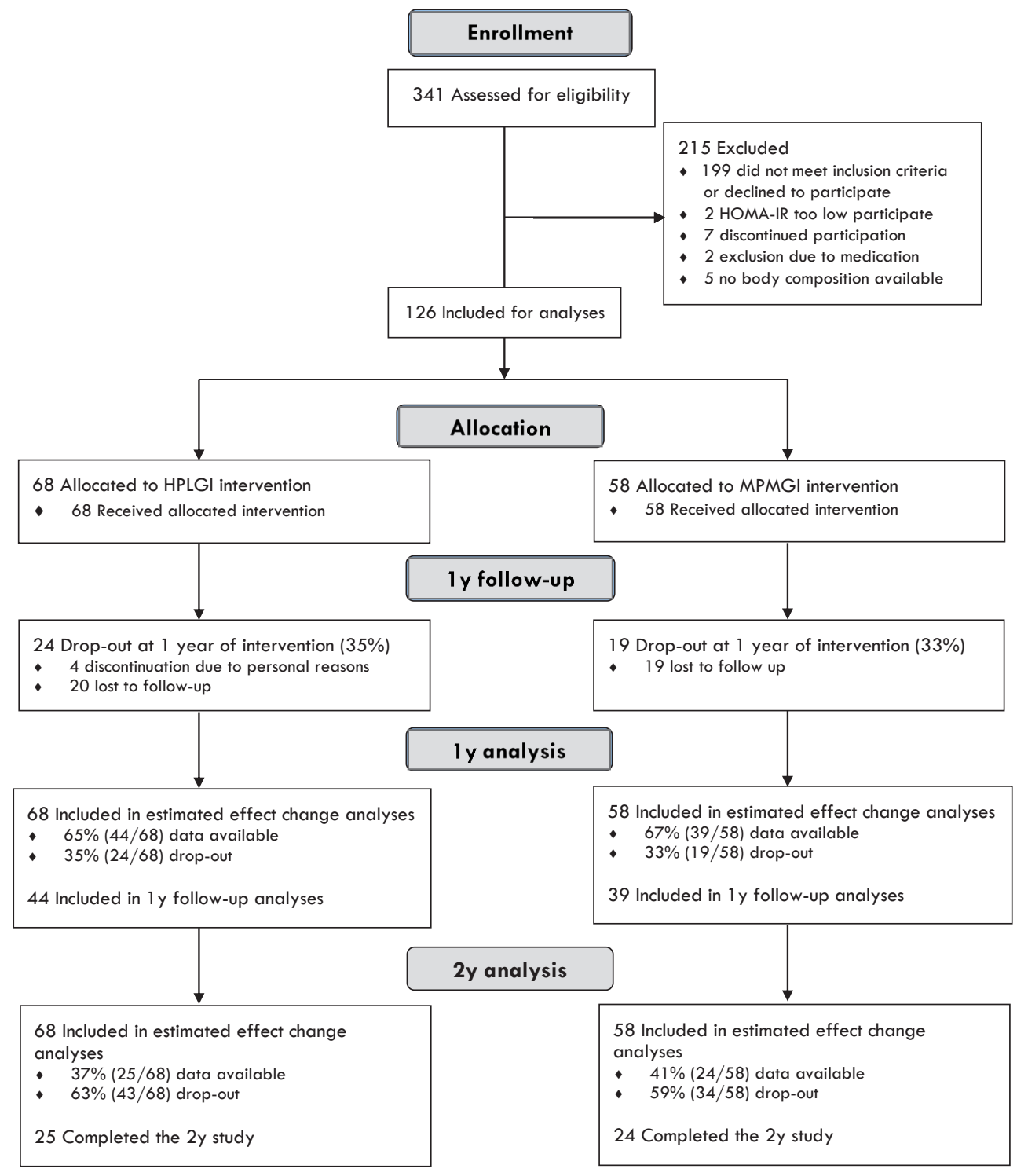

Figure 4.1: Study flowchart. 


\section{Effects of the dietary intervention on IR, metabolic and anthropometric parameters}

No significant differences were observed between the two intervention groups regarding insulin resistance, parameters of glucose metabolism, lipid metabolism, inflammation, liver enzymes, anthropometric characteristics, or lifestyle factors at any timepoint.

\section{Post-hoc observations}

Regarding dietary observations, the total energy requirements for adolescents with corresponding ages, heights and weights to the study participants is 10.7-15.7 $\mathrm{MJ} / \mathrm{d}$ for girls and boys (WHO-formula), the reported total energy intake was 5.9-7.0 $\mathrm{MJ} / \mathrm{d}$ in this study ${ }^{27}$. Intention-to-treat analyses showed a significant decrease in $\mathrm{Gl}$ and $\mathrm{GL}$ after $2 \mathrm{y}$, but not $1 \mathrm{y}$, of study participation (Table 4.1A). Cognitive dietary restraint scores on the TFEQ increased significantly after $1 \mathrm{y}$ in the completers subset, and after $2 \mathrm{y}$ in the ITT analyses (Tables 4.1A \& 4.2A).

With respect to physical activity (PA), expressed as accelerometry counts per minute, and minutes spent in moderate, vigorous and moderate-to-vigorous, a significant increase at 1 and $2 \mathrm{y}$, was observed, while sedentary time decreased (Tables 4.1A \& 2A).

Considering anthropometric changes, mean age- and sex-corrected BMI z-score was significantly reduced, while pubertal stage, height, weight, BMI, fat free mass and fat mass increased (Tables 4.1B \& 4.2B).

Regarding insulin resistance and further metabolic changes, fasting blood glucose concentration increased. And at 2y, but not after 1y, HOMA-IR was significantly higher than at baseline. After 1y mean heart rate was decreased. Differently from the set of adolescents that completed the intervention, the intention-to-treat analyses showed a significant increase in ALT concentration.

\section{Post-hoc associations with changes in BMI z-score}

Change in BMI z-score was negatively associated with change in cognitive restraint and positively with susceptibility to hunger on the TFEQ, after correcting for change in Tanner stage (Table 4.3). HOMA-IR was positively related to change in GI (Table 4.3). Change in BMI z-score was positively related to change in HOMA-IR after correcting for changes in Tanner G/M stage and BMI z-score at baseline (Figure 4.2). Other lifestyle variables were not significantly associated with change in BMI z-score or HOMA-IR, nor with changes in anthropometric characteristics or cardiovascular health parameters, after correcting for relevant confounders. 
TABLE 4.1A: Estimated effect changes in food intake, physical activity and sleep parameters after 1 and 2 years of intervention

\begin{tabular}{|c|c|c|c|}
\hline & \multicolumn{3}{|c|}{ Baseline } \\
\hline & Whole group & $\underline{\mathrm{HP}}$ & MP \\
\hline & $(n=126)$ & $(n=68)$ & $(n=58)$ \\
\hline Outcome & mean $\pm S D$ & mean $\pm S D$ & mean $\pm S D$ \\
\hline \multicolumn{4}{|l|}{ Food intake } \\
\hline Glycaemic Index & $52.4 \pm 8.2$ & $50.9 \pm 7.7$ & $54.3 \pm 8.7$ \\
\hline Glycaemic Load & $97.9 \pm 35.4$ & $93.4 \pm 30.9$ & $103.0 \pm 32.8$ \\
\hline Energy intake $(\mathrm{MJ} / \mathrm{d})$ & $7.0 \pm 2.0$ & $6.7 \pm 1.6$ & $7.3 \pm 2.3$ \\
\hline Protein $(g / d)$ & $71.0 \pm 18.2$ & $70.0 \pm 19.2$ & $70.9 \pm 17.4$ \\
\hline Protein (En\%) & $17.2 \pm 3.0$ & $17.5 \pm 2.4$ & $16.9 \pm 3.7$ \\
\hline Fat $(g / d)$ & $67.8 \pm 26.1$ & $65.9 \pm 23.5$ & $70.2 \pm 29.3$ \\
\hline Fat (En\%) & $35.7 \pm 7.4$ & $35.9 \pm 7.8$ & $35.4 \pm 6.9$ \\
\hline Carbohydrate (g/d) & $187.2 \pm 60.7$ & $176.3 \pm 46.0$ & $200.9 \pm 74.2$ \\
\hline Carbohydrate (En\%) & $45.1 \pm 7.1$ & $44.7 \pm 7.8$ & $45.6 \pm 6.1$ \\
\hline Fibre $(\mathrm{g} / \mathrm{d})$ & $14.0 \pm 5.5$ & $13.9 \pm 5.0$ & $14.1 \pm 6.2$ \\
\hline \multicolumn{4}{|l|}{ Food intake parameters } \\
\hline TFEQ cognitive restraint of eating & $11.0 \pm 3.8$ & $10.6 \pm 3.6$ & $11.5 \pm 4.1$ \\
\hline TFEQ disinhibition & $6.2 \pm 3.3$ & $6.7 \pm 3.5$ & $5.4 \pm 3.0$ \\
\hline TFEQ hunger & $5.4 \pm 3.3$ & $5.8 \pm 3.7$ & $4.9 \pm 2.5$ \\
\hline \multicolumn{4}{|l|}{ Physical activity parameters } \\
\hline Baecke School & $2.5 \pm 0.4$ & $2.4 \pm 0.3$ & $2.6 \pm 0.4$ \\
\hline Baecke Sport & $2.8 \pm 0.5$ & $2.7 \pm 0.5$ & $2.8 \pm 0.5$ \\
\hline Baecke Leisure & $2.8 \pm 0.7$ & $2.9 \pm 0.6$ & $2.8 \pm 0.7$ \\
\hline Baecke total score & $8.1 \pm 1.0$ & $8.0 \pm 1.0$ & $8.2 \pm 1.1$ \\
\hline Accelerometry counts (kcpd) & $297.5 \pm 101.5$ & $309.4 \pm 99.9$ & $279.4 \pm 103.1$ \\
\hline Accelerometry counts (cpm) & $306.7 \pm 108.5$ & $316.6 \pm 101.9$ & $291.0 \pm 118.7$ \\
\hline Sedentary behaviour ( $\mathrm{min} / \mathrm{d})$ & $631.6 \pm 114.4$ & $617.0 \pm 105.8$ & $653.4 \pm 125.0$ \\
\hline Light PA (min/d) & $323.7 \pm 71.0$ & $335.0 \pm 7.8$ & $306.7 \pm 69.1$ \\
\hline Moderate PA $(\mathrm{min} / \mathrm{d})$ & $21.4 \pm 14.7$ & $22.2 \pm 13.3$ & $20.3 \pm 16.9$ \\
\hline Vigorous PA (min/d) & $4.7 \pm 5.0$ & $4.7 \pm 4.8$ & $4.7 \pm 5.4$ \\
\hline Moderate-to-vigorous PA ( $\mathrm{min} / \mathrm{d}$ ) & $26.1 \pm 17.5$ & $26.9 \pm 16.4$ & $25.1 \pm 19.2$ \\
\hline
\end{tabular}

HP: High-Protein Low-Glycaemic Index; MP: Medium-Protein Medium-Glycaemic Index; BMI = Body Mass Index; FFM = fat free mass; FM = fat mass; $S B P=$ systolic blood pressure; $D B P=$ diastolic blood pressure; $H R=$ heart rate; $H O M A-I R=$ Homeostatic Model Assessment of Insulin Resistance (glucose $(\mathrm{mmol} / \mathrm{L}) /$ insulin $(\mathrm{mU} / \mathrm{L}) * 22,5^{34} ; \mathrm{HDL}$ cholesterol = high-density lipoprotein cholesterol; LDL cholesterol = low-density lipoprotein cholesterol; TG = triacylglycerides; $C R P=C$-reactive protein; $A S T=$ aspartate aminotransferase; $A L T=$ alanine aminotransferase; En $\%=$ percentage of total energy intake; TFEQ = Three Factor Eating Questionnaire ${ }^{35} ; \mathrm{cpd}=$ counts per day $; \mathrm{cpm}=$ counts per minute; $P A=$ Physical Activity. Estimated effect changes are based upon imputated datasets. $P$-values are based upon comparison with baseline values. ${ }^{*} p<0.05 ;{ }^{* *} p<0.01$. 


\begin{tabular}{|c|c|c|c|c|c|}
\hline \multicolumn{3}{|c|}{$\begin{array}{c}\text { Estimated effect changes } \\
\text { after } 1 y \text { (after multiple imputation) }\end{array}$} & \multicolumn{3}{|c|}{$\begin{array}{l}\text { Estimated effect changes } \\
\text { after } 2 y \text { (after multiple imputation) }\end{array}$} \\
\hline Whole group & $\underline{\mathrm{HP}}$ & MP & Whole group & $\underline{\mathrm{HP}}$ & MP \\
\hline$(n=126)$ & $(n=68)$ & $(n=58)$ & $(n=126)$ & $(n=68)$ & $(n=58)$ \\
\hline$\Delta(95 \% C l)$ & $\Delta(95 \% \mathrm{Cl})$ & $\Delta(95 \% \mathrm{Cl})$ & $\Delta(95 \% \mathrm{Cl})$ & $\Delta(95 \% \mathrm{Cl})$ & $\Delta(95 \% \mathrm{Cl})$ \\
\hline$-1.2(-4.7,2.3)$ & $0(-4.6,4.6)$ & $-2.7(-7.9,2.6)$ & $-2.0(-3.7,-0.3)^{*}$ & $-1.4(-3.5,0.6)$ & $-2.8(-5.4,-0.2)^{*}$ \\
\hline$-13.6(-27.1,0.0)$ & $-8.2(-24.7,8.2)$ & $-20.3(-41.8,1.1)$ & $-10.1(-19.4,-0.8)^{*}$ & $-6.8(-17.2,3.7)$ & $-14.5(-30.1,1.0)$ \\
\hline$-1.0(-1.6,-0.4)^{* *}$ & $-0.9(-1.7,-0.1)^{*}$ & $-1.2(-2.1,-0.3)^{*}$ & $-0.2(-0.6,0.2)$ & $0.0(-0.5,0.5)$ & $-0.6(-1.6,0.1)$ \\
\hline$-5.3(-10.7,0.2)$ & $-6.0(-13.7,-1.8)$ & $-4.4(-11.7,2.8)$ & $-2.8(-6.8,1.1)$ & $-1.7(-6.5,3.0)$ & $-4.3(-10.9,2.2)$ \\
\hline $1.8(-0.2,3.9)$ & $1.8(-1.1,4.7)$ & $1.8(-0.7,4.4)$ & $-0.2(-1.4,1.1)$ & $-0.5(-2.2,1.2)$ & $0.3(-1.7,2.2)$ \\
\hline$-10.7(-19.1,-2.4)^{*}$ & $-9.4(-20.3,1.5)$ & $-12.4(-25.0,0.1)$ & $0.1(-5.1,5.2)$ & $2.3(-3.4,8.1)$ & $-2.9(-11.2,5.3)$ \\
\hline $0.8(-4.6,6.2)$ & $0.8(-6.6,8.2)$ & $0.8(-6.2,7.8)$ & $1.3(-1.8,4.4)$ & $1.4(-2.5,5.2)$ & $1.2(-3.4,5.9)$ \\
\hline$-29.1(-49.4,-8.7)^{* *}$ & $-25.2(-48.8,-1.6)$ & $-34.0(-68.3,0.4)$ & $-10.1(-24.6,4.4)$ & $-4.0(-21.1,13.2)$ & $-18.4(-41.3,4.5)$ \\
\hline $0.9(-5.1,6.8)$ & $0.9(-7.0,8.9)$ & $0.8(-6.7,8.3)$ & $-0.5(-4.4,3.5)$ & $-0.8(-5.7,4.1)$ & $0.0(-5.9,6.0)$ \\
\hline $0.3(-1.4,1.9)$ & $1.0(-1.1,3.2)$ & $-0.7(-2.9,1.4)$ & $0.1(-1.7,1.9)$ & $-0.1(-2.4,2.1)$ & $0.4(-2.5,3.3)$ \\
\hline $0.9(-0.5,2.3)$ & $1.2(-0.6,3.0)$ & $0.6(-1.5,2.6)$ & $2.1(0.7,3.5)^{* *}$ & $2.4(0.6,4.1)^{* *}$ & $1.8(-0.3,3.9)$ \\
\hline $0.2(-0.8,1.2)$ & $-0.1(-1.5,1.3)$ & $0.6(-0.9,2.0)$ & $1.5(-0.2,3.2)$ & $0.7(-1.4,2.7)$ & $2.7(0.3,5.1)^{*}$ \\
\hline$-0.5(-2.0,0.9)$ & $-1.0(-3.1,1.0$ & $0.1(-1.5,1.8)$ & $1.4(-0.5,3.4)$ & $0.8(-1.5,3.1)$ & $2.3(-0.4,5.0)$ \\
\hline $0.0(-0.1,0.1)$ & $0.1(0.0,0.2)$ & $-0.1(-0.2,0.1)$ & $-0.1(-0.3,0.0)$ & $-0.0(-0.2,0.1)$ & $-0.2(-0.4,-0.0)^{*}$ \\
\hline $0.1(-0.1,0.2)$ & $0.1(-0.1,0.3)$ & $0.1(-0.1,0.3)$ & $-0.1(-0.3,0.1)$ & $-0.1(-0.3,0.1)$ & $-0.2(-0.4,0.1)$ \\
\hline $0.0(-0.1,0.2)$ & $0.0(-0.2,0.2)$ & $0.0(-0.2,0.3)$ & $-0.0(-0.2,0.1)$ & $-0.1(-0.3,0.2)$ & $-0.0(-0.3,0.2)$ \\
\hline $0.1(-0.1,0.4)$ & $0.2(-0.2,0.5)$ & $0.1(-0.3,0.4)$ & $-0.3(-0.5,-0.0)$ & $-0.1(-0.5,0.2)$ & $-0.4(-0.8,-0.0)$ \\
\hline $33.0(5.3,60.7)^{* *}$ & $27.7(-10.5,65.9)$ & $39.5(-0.3,79.4)$ & $29.6(-11.8,71.1)$ & $4.2(-46.4,54.8)$ & $60.9(5.7,116.0)^{*}$ \\
\hline $106.5(71.2,141.7)^{* *}$ & $104.2(60.2,148.3)^{* *}$ & $109.2(58.3,160.1)^{* *}$ & $76.5(33.6,119.5)^{* *}$ & $51.7(1.1,102.3)^{*}$ & $107.1(48.2,166.0)^{* *}$ \\
\hline$-177.2(-211.4,-143.0)^{* *}$ & $-165.3(-210.1,-120.6)^{* *}$ & $-191.8(-241.7,-141.9)^{* *}$ & $-156.7(-190.1,-123.3)^{* *}$ & $-138.9(-180.7,-97.1)^{* *}$ & $-178.5(-224.1,-132.9)^{* *}$ \\
\hline$-35.7(-58.0,-13.3)^{* *}$ & $-40.6(69.9,-11.3)^{* *}$ & $-29.6(-59.9,0.8)$ & $-19.0(-48.8,10.9)$ & $-37.1(-71.8,-2.4)^{*}$ & $3.4(-37.9,44.6)$ \\
\hline $7.3(3.9,10.7)^{* *}$ & $6.7(2.3,11.1)^{* *}$ & $8.0(2.8,13.1)^{* *}$ & $5.0(0.1,9.9)^{*}$ & $3.0(-2.7,8.7)$ & $7.4(0.8,14 .)^{*}$ \\
\hline $5.3(2.8,7.8)^{* *}$ & $5.7(2.3,9.1)^{* *}$ & $4.8(1.5,8.2)^{* *}$ & $3.3(1.1,5.5)^{* *}$ & $2.8(0.0,5.6)^{*}$ & $3.8(0.8,6.9)^{*}$ \\
\hline $12.5(8.0,16.9)^{* *}$ & $12.3(6.4,18.2)^{* *}$ & $12.6(5.9,19.4)^{* *}$ & $8.2(3.1,13.4)^{* *}$ & $5.7(3.7,12.1)$ & $11.4(4.0,18.8)^{* *}$ \\
\hline
\end{tabular}


TABLE 4.1B: Estimated effect changes in anthropometric characteristics, body composition, blood pressure, and parameters of glucose metabolism, lipids, liver enzymes and inflammation and blood pressure after 1 and 2 years of intervention

\begin{tabular}{|c|c|c|c|}
\hline & \multicolumn{3}{|c|}{ Baseline } \\
\hline & Whole group & $\underline{\mathrm{HP}}$ & $\underline{M P}$ \\
\hline & $(n=126)$ & $(n=68)$ & $(n=58)$ \\
\hline Outcome & mean $\pm S D$ & mean $\pm S D$ & mean $\pm S D$ \\
\hline \multicolumn{4}{|l|}{ General characteristics } \\
\hline Girls n (\%) & $74(58.7 \%)$ & $39(57.4 \%)$ & $35(60.3 \%)$ \\
\hline Age (yr) & $13.6 \pm 2.2$ & $13.7 \pm 2.4$ & $13.4 \pm 2.0$ \\
\hline Tanner stage & $3(2-4)$ & $3(2-5)$ & $3(2-4)$ \\
\hline \multicolumn{4}{|c|}{ Anthropometric characteristics } \\
\hline Height (m) & $1.61 \pm 0.11$ & $1.61 \pm 0.11$ & $1.60 \pm 0.10$ \\
\hline Weight $(\mathrm{kg})$ & $78.0 \pm 19.7$ & $80.0 \pm 20.9$ & $75.7 \pm 18.2$ \\
\hline $\mathrm{BMI}(\mathrm{kg} / \mathrm{m} 2)$ & $29.8 \pm 4.9$ & $30.1 \pm 5.1$ & $29.3 \pm 4.6$ \\
\hline BMI z-score (SD) & $3.04 \pm 0.66$ & $3.10 \pm 0.69$ & $2.97 \pm 0.63$ \\
\hline Fat free mass $(\mathrm{kg})$ & $46.9 \pm 11.5$ & $47.5 \pm 12.3$ & $46.2 \pm 10.5$ \\
\hline Fat mass $(\mathrm{kg})$ & $31.0 \pm 11.8$ & $32.4 \pm 12.3$ & $29.3 \pm 11.0$ \\
\hline Fat mass (\%) & $39.2 \pm 8.11$ & $40.0 \pm 7.4$ & $38.2 \pm 8.9$ \\
\hline \multicolumn{4}{|l|}{ Blood pressure } \\
\hline $\mathrm{SBP}(\mathrm{mmHg})$ & $116,5 \pm 12.4$ & $116.8 \pm 12.9$ & $116.0 \pm 11.9$ \\
\hline $\mathrm{DBP}(\mathrm{mmHg})$ & $66.6 \pm 7.6$ & $67.3 \pm 8.0$ & $65.7 \pm 7.1$ \\
\hline $\mathrm{HR}(\mathrm{bpm})$ & $75.3 \pm 11.7$ & $76.1 \pm 13.3$ & $74.4 \pm 9.5$ \\
\hline \multicolumn{4}{|c|}{ Parameters of glucose metabolism, lipids, inflammation and liver enzymes } \\
\hline Glucose $(\mathrm{mmol} / \mathrm{L})$ & $4.6 \pm 0.7$ & $4.6 \pm 0.7$ & $4.5 \pm 0.7$ \\
\hline Insulin $(\mathrm{pmol} / \mathrm{L})$ & $109.6 \pm 74.2$ & $107.2 \pm 51.5$ & $112.4 \pm 94.6$ \\
\hline HOMA-IR & $3.35 \pm 1.80$ & $3.44 \pm 1.66$ & $3.24 \pm 1.96$ \\
\hline $\mathrm{HbA1c}(\mathrm{mmol} / \mathrm{mol})$ & $32.7 \pm 2.7$ & $32.7 \pm 2.9$ & $32.8 \pm 2.5$ \\
\hline C-peptide (nmol/L) & $0.9 \pm 0.3$ & $0.9 \pm 0.3$ & $0.9 \pm 0.3$ \\
\hline Total cholesterol $(\mathrm{mmol} / \mathrm{L})$ & $4.1 \pm 0.8$ & $4.1 \pm 0.7$ & $4.2 \pm 0.8$ \\
\hline HDL cholesterol $(\mathrm{mmol} / \mathrm{L})$ & $1.3 \pm 0.3$ & $1.3 \pm 0.3$ & $1.3 \pm 0.3$ \\
\hline LDL cholesterol ( $\mathrm{mmol} / \mathrm{L})$ & $2.4 \pm 0.6$ & $2.4 \pm 0.6$ & $2.4 \pm 0.7$ \\
\hline TG $(\mathrm{mmol} / \mathrm{L})$ & $1.1 \pm 0.5$ & $1.1 \pm 0.5$ & $1.1 \pm 0.6$ \\
\hline $\mathrm{CRP}(\mathrm{mg} / \mathrm{L})$ & $2.9 \pm 3.1$ & $2.9 \pm 2.7$ & $3.0 \pm 3.5$ \\
\hline AST (U/L) & $25.1 \pm 8.1$ & $23.9 \pm 7.4$ & $26.6 \pm 8.8$ \\
\hline $\operatorname{ALT}(\mathrm{U} / \mathrm{L})$ & $24.7 \pm 14.6$ & $23.5 \pm 13.8$ & $26.2 \pm 15.5$ \\
\hline
\end{tabular}

HP: High-Protein Low-Glycaemic Index; MP: Medium-Protein Medium-Glycaemic Index; BMI = Body Mass Index; FFM = fat free mass; FM = fat mass; $S B P=$ systolic blood pressure; $D B P=$ diastolic blood pressure; $H R=$ heart rate; $H O M A-I R=$ Homeostatic Model Assessment of Insulin Resistance $(g / u c o s e$ $(\mathrm{mmol} / \mathrm{L}) /$ insulin $(\mathrm{mU} / \mathrm{L}){ }^{*} 22,5^{34} ; \mathrm{HDL}$ cholesterol = high-density lipoprotein cholesterol; LDL cholesterol = low-density lipoprotein cholesterol; $T G=$ triacylglycerides; $C R P=C$-reactive protein; $A S T=$ aspartate aminotransferase; $A L T=$ alanine aminotransferase; En $\%=$ percentage of total energy intake; TFEQ = Three Factor Eating Questionnaire ${ }^{35} ; c p d=$ counts per day; $c p m=$ counts per minute; $P A=$ Physical Activity. Estimated effect changes are based upon imputated datasets. $P$-values are based upon comparison with baseline values. ${ }^{*} p<0.05 ;{ }^{* *} p<0.01$. 


\begin{tabular}{|c|c|c|c|c|c|}
\hline \multicolumn{3}{|c|}{$\begin{array}{l}\text { Estimated effect changes } \\
\text { after } 1 y \text { (after multiple imputation) }\end{array}$} & \multicolumn{3}{|c|}{$\begin{array}{c}\text { Estimated effect changes } \\
\text { after } 2 y \text { (after multiple imputation) }\end{array}$} \\
\hline Whole group & $\mathrm{HP}$ & MP & Whole group & $\mathrm{HP}$ & MP \\
\hline$(n=126)$ & $(n=68)$ & $(n=58)$ & $(n=126)$ & $(n=68)$ & $(n=58)$ \\
\hline$\Delta(95 \% C l)$ & $\Delta(95 \% C l)$ & $\Delta(95 \% C l)$ & $\Delta(95 \% C l)$ & $\Delta(95 \% C l)$ & $\Delta(95 \% C l)$ \\
\hline $1.1(1.1,1.2)^{* *}$ & $1.1(1.1,1.2)^{* *}$ & $1.1(1.1,1.2)^{* *}$ & $2.2(2.1,2.3)^{* *}$ & $2.2(2.0,2.3)^{* *}$ & $2.2(2.0,2.3)^{* *}$ \\
\hline $0.5(0.4,0.7)^{* *}$ & $0.6(0.3,0.8)^{* *}$ & $0.5(0.3,0.8)^{* *}$ & $0.7(0.5,1.0)^{* *}$ & $0.8(0.4,1.2)^{* *}$ & $0.7(0.3,1.1)^{* *}$ \\
\hline $0.05(0.04,0.06)^{* *}$ & $0.05(0.04,0.06)^{* *}$ & $0.05(0.04,0.07)^{* *}$ & $0.08(0.06,0.10)^{* *}$ & $0.08(0.05,0.11)^{* *}$ & $0.09(0.06,0.11)^{* * *}$ \\
\hline $9.3(4.2,14.5)^{* *}$ & $8.3(1.1,15.5)^{*}$ & $10.6(3.6,17.6)^{* *}$ & $3,8(-0.4,8.0)$ & $4.4(-1.5,10.4)$ & $3.0(-2.9,8.9)$ \\
\hline $1.7(0.0,3.5)$ & $1.3(-1.1,3.6)$ & $2.3(-0.3,4.9)$ & $-0.4(-1.4,0.6)$ & $-0.33(-1.8,1.2)$ & $-0.5(-1.8,0.8)$ \\
\hline$-0.16(-0.24,-0.07)^{* *}$ & $-0.22(-0.33,-0.10)^{* *}$ & $-0.09(-0.21,0.03)$ & $-0.19(-0.36,-0.02)^{*}$ & $-0.16(-0.36,0.04)$ & $-0.22(-0.46,0.01)$ \\
\hline $3.3(2.2,4.4)^{* *}$ & $3.0(1.5,4.5)^{* *}$ & $3.6(2.0,5.3)^{* *}$ & $6.4(4.2,8.7)^{* *}$ & $6.8(3.7,9.9)^{* *}$ & $6.0(3.3,8.7)^{* *}$ \\
\hline $1.8(0.4,3.2)^{*}$ & $1.0(-0.9,3.0)$ & $2.7(0.7,4.7)^{* *}$ & $4.6(1.5,7.6)^{* *}$ & $5.0(1.4,8.6)^{* *}$ & $4.0(-0.3,8.4)$ \\
\hline$-0.8(-3.3,1.7)$ & $-1.1(-4.7,2.5)$ & $-0.5(-3.8,2.9)$ & $1.6(-0.2,3.5)$ & $1.4(-0.9,3.7)$ & $2.0(-0.8,4.8)$ \\
\hline $1.3(-1.5,1.3)$ & $0.7(-3.1,4.5)$ & $2.0(-2.1,6.1)$ & $3.2(-0.7,7.3)$ & $4.2(-0.9,9.2)$ & $2.3(-3.0,7.5)$ \\
\hline$-0.8(-2.6,1.1)$ & $-0.7(-3.2,1.8)$ & $-0.8(-3.2,1.5)$ & $2.0(-0.8,4.7)$ & $2.4(-1.2,6.0)$ & $1.4(-2.3,5.1)$ \\
\hline$-2.3(-4.4,-0.3)^{*}$ & $-3.0(-5.9,-0.2)^{*}$ & $-1.5(-4.3,1.3)$ & $-0.5(-4.7,3.8)$ & $-1.2(-6.3,3.9)$ & $0.4(-4.9,5.8)$ \\
\hline $0.2(0.0,0.4)^{*}$ & $0.2(-0.1,0.4)$ & $0.2(-0.0,0.5)$ & $0.4(0.2,0.6)^{* *}$ & $0.3(0.1,0.6)^{*}$ & $0.4(0.1,0.7)^{* *}$ \\
\hline$-0.2(-18.5,14.4)$ & $-5.8(-25.6,13.8)$ & $2.5(-22.6,27.6)$ & $21.2(-10.1,52.5)$ & $28.1(-11.4,67.6)$ & $13.2(-25.5,51.9)$ \\
\hline $0.31(-0.30,0.92)$ & $0.19(-0.55,0.94)$ & $0.45(-0.47,1.37)$ & $0.87(0.23,1.52)^{* *}$ & $0.85(-0.00,1.72)$ & $0.89(-0.00,1.78)$ \\
\hline $0.3(-0.4,1.0)$ & $0.1(-0.8,1.0)$ & $0.5(-0.4,1.5)$ & $0.8(-0.0,1.6)$ & $0.7(-0.3,1.7)$ & $0.9(-0.2,2.0)$ \\
\hline $0.0(-0.1,0.1)$ & $0.0(-0.1,0.1)$ & $0.0(-0.1,0.1)$ & $0.1(-0.0,1.6)$ & $0.1(-0.0,0.2)$ & $0.0(-0.1,0.2)$ \\
\hline$-0.1(-0.2,0.1)$ & $0.0(-0.2,0.2)$ & $-0.1(-0.3,0.1)$ & $-0.1(-0.3,0.1)$ & $-0.0(-0.3,0.2)$ & $-0.1(-0.3,0.1)$ \\
\hline $0.0(-0.1,0.0)$ & $0.0(-0.1,0.1)$ & $0.0(-0.1,0.1)$ & $-0.0(-0.1,0.1)$ & $-0.0(-0.2,0.1)$ & $0.0(-0.1,0.1)$ \\
\hline $0.0(-0.2,0.1)$ & $0.0(-0.2,0.1)$ & $-0.1(-0.2,0.1)$ & $-0.1(-0.3,0.1)$ & $-0.0(-0.3,0.2)$ & $-0.1(-0.4,0.1)$ \\
\hline $0.0(-1.9,0.5)$ & $0.0(-0.1,0.1)$ & $0.0(-0.2,0.1)$ & $-0.0(-0.2,0.1)$ & $0.0(-0.1,0.2)$ & $-0.1(-0.2,0.1)$ \\
\hline$-0.7(-1.9,0.5)$ & $-0.5(-2.1,1.0)$ & $-0.9(-2.5,0.8)$ & $1.1(-0.6,2.7)$ & $1.4(-0.6,3.4)$ & $0.6(-1.7,2.9)$ \\
\hline $2.3(-2.5,7.1)$ & $1.4(-4.2,7.1)$ & $3.4(-4.6,11.4)$ & $-0.1(2.7,2.6)$ & $0.6(-2.6,3.8)$ & $-0.9(-4.6,2.8)$ \\
\hline $4.1(0.3,7.8)^{*}$ & $5.3(0.5,10.1)^{*}$ & $2.6(-2.8,8.0)$ & $3.7(-1.1,8.6)$ & $5.7(-0.3,11.6)$ & $1.4(-4.9,7.8)$ \\
\hline
\end{tabular}


TABLE 4.2A: Food intake and physical activity parameters at baseline and after one year of PREVIEW intervention $(n=83)$

\begin{tabular}{|c|c|c|c|}
\hline & \multicolumn{3}{|c|}{ Baseline } \\
\hline & $\frac{\text { Whole group }}{(n=83)}$ & $\frac{\mathrm{HP}}{(n=44)}$ & $\frac{\mathrm{MP}}{(n=39)}$ \\
\hline Outcome & mean $\pm S D$ & mean $\pm S D$ & mean $\pm S D$ \\
\hline \multicolumn{4}{|l|}{ Food intake } \\
\hline Glycaemic Index & $51.9 \pm 8.9$ & $50.2 \pm 8.7$ & $54.3 \pm 8.7$ \\
\hline Glycaemic Load & $96.4 \pm 36.2$ & $87.0 \pm 28.3$ & $109.1 \pm 42.1$ \\
\hline Energy intake (MJ/d) & $6.8 \pm 0.1$ & $6.5 \pm 1.9$ & $7.3 \pm 2.3$ \\
\hline Protein (g/d) & $73.9 \pm 24.9$ & $76.2 \pm 27.9$ & $70.9 \pm 17.4$ \\
\hline Protein (En\%) & $17.9 \pm 14.0$ & $19.1 \pm 18.0$ & $16.4 \pm 3.8$ \\
\hline Fat $(\mathrm{g} / \mathrm{d})$ & $68.9 \pm 26.5$ & $68.0 \pm 24.7$ & $70.2 \pm 29.3$ \\
\hline Fat (En\%) & $37.6 \pm 19.3$ & $38.4 \pm 24.5$ & $36.7 \pm 6.8$ \\
\hline Carbohydrate (g/d) & $183.0 \pm 63.4$ & $169.7 \pm 51.4$ & $200.9 \pm 74.2$ \\
\hline Carbohydrate (En\%) & $44.4 \pm 7.1$ & $42.5 \pm 7.7$ & $46.7 \pm 6.2$ \\
\hline Fibre $(\mathrm{g} / \mathrm{d})$ & $15.4 \pm 6.5$ & $14.9 \pm 7.2$ & $16.1 \pm 5.4$ \\
\hline \multicolumn{4}{|l|}{ Food intake parameters } \\
\hline TFEQ cognitive restraint of eating & $10.99 \pm 3.795$ & $10.6 \pm 3.573$ & $11.53 \pm 4.091$ \\
\hline TFEQ disinhibition & $6.15 \pm 3.319$ & $6.7 \pm 3.475$ & $5.37 \pm 2.965$ \\
\hline TFEQ hunger & $5.41 \pm 3.286$ & $5.77 \pm 3.721$ & $4.90 \pm 2.51$ \\
\hline \multicolumn{4}{|l|}{ Physical activity parameters } \\
\hline Baecke School & $2.5 \pm 0.4$ & $2.4 \pm 0.3$ & $2.6 \pm 0.4$ \\
\hline Baecke Sport & $2.8 \pm 0.5$ & $2.7 \pm 0.5$ & $2.8 \pm 0.5$ \\
\hline Baecke Leisure & $2.9 \pm 0.6$ & $2.9 \pm 0.6$ & $2.8 \pm 0.7$ \\
\hline Baecke total score & $8.2 \pm 1.0$ & $8.0 \pm 1.0$ & $8.2 \pm 1.1$ \\
\hline Accelerometry counts (kcpd) & $279.5 \pm 107.8$ & $309.4 \pm 99.9$ & $279.4 \pm 103.1$ \\
\hline Accelerometry counts (cpm) & $285.5 \pm 113.1$ & $318.0 \pm 101.1$ & $291.2 \pm 116.8$ \\
\hline Sedentary behaviour (min) & $652.7 \pm 116.4$ & $617.0 \pm 105.8$ & $653.4 \pm 125.0$ \\
\hline Light PA (min) & $314.3 \pm 65.6$ & $335.0 \pm 70.8$ & $306.7 \pm 69.1$ \\
\hline Moderate PA (min) & $17.6 \pm 11.4$ & $22.2 \pm 13.3$ & $20.3 \pm 16.9$ \\
\hline Vigorous PA (min) & $4.1 \pm 5.4$ & $4.7 \pm 4.8$ & $4.7 \pm 5.4$ \\
\hline Moderate-to-vigorous PA (min) & $21.7 \pm 14.5$ & $26.9 \pm 16.4$ & $25.1 \pm 19.2$ \\
\hline
\end{tabular}

HP: High-Protein Low-Glycaemic Index; MP: Medium-Protein Medium-Glycaemic Index; BMI = Body Mass Index; FFM = fat free mass; $F M=$ fat mass; $S B P=$ systolic blood pressure; $D B P=$ diastolic blood pressure; $H R=$ heart rate; HOMA-IR= Homeostatic Model Assessment of Insulin Resistance (glucose ( $\mathrm{mmol} / \mathrm{L})$ /insulin $(\mathrm{mU} / \mathrm{L}) * 22,5^{34} ; \mathrm{HDL}$ cholesterol=high-densitylipoprotein cholesterol; $L D L$ cholesterol = low-density lipoprotein cholesterol; TG = triacylglycerides; $C R P=C$-reactive protein; $A S T$ = aspartate aminotransferase;

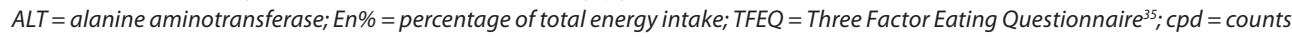
perday $; \mathrm{cpm}=$ counts per minute; $P A=$ Physical Activity. Estimated effect changes are based upon imputated datasets. $P$-values are based upon comparison with baseline values. ${ }^{*} p<0.05 ;{ }^{* *} p<0.01$. 


\begin{tabular}{|c|c|c|}
\hline \multicolumn{3}{|c|}{ 1y follow-up } \\
\hline Whole group & $\underline{\mathrm{HP}}$ & $\underline{\mathrm{MP}}$ \\
\hline$(n=83)$ & $(n=44)$ & $(n=39)$ \\
\hline mean $\pm S D$ & mean $\pm S D$ & mean $\pm S D$ \\
\hline $52.4 \pm 7.0$ & $52.7 \pm 6.1$ & $52.2 \pm 7.9$ \\
\hline $84.1 \pm 24.7$ & $72.8 \pm 18.0$ & $92.9 \pm 26.2$ \\
\hline $5.9 \pm 1.3^{* *}$ & $5.4 \pm 1.2$ & $6.4 \pm 1.2^{*}$ \\
\hline $65.0 \pm 12.8$ & $61.1 \pm 12.1$ & $67.9 \pm 13.0$ \\
\hline $18.9 \pm 3.1$ & $19.4 \pm 2.8$ & $18.4 \pm 3.4$ \\
\hline $55.0 \pm 18.6^{* *}$ & $51.8 \pm 21.8$ & $57.4 \pm 16.2^{* *}$ \\
\hline $33.6 \pm 7.5$ & $34.1 \pm 9.6$ & $33.2 \pm 5.8$ \\
\hline $158.6 \pm 37.9^{*}$ & $137.9 \pm 25.2^{*}$ & $174.6 \pm 39.0$ \\
\hline $45.7 \pm 7.7$ & $44.4 \pm 8.8$ & $46.7 \pm 6.8$ \\
\hline $14.7 \pm 4.1$ & $14.2 \pm 3.8$ & $15.2 \pm 4.4$ \\
\hline $12.2 \pm 3.6^{*}$ & $11.6 \pm 3.7$ & $12.8 \pm 3.6^{*}$ \\
\hline $5.9 \pm 3.6$ & $6.1 \pm 3.7$ & $5.7 \pm 3.5$ \\
\hline $4.7 \pm 3.9$ & $4.3 \pm 4.2$ & $5.0 \pm 3.7$ \\
\hline $2.5 \pm 0.4$ & $2.5 \pm 0.4$ & $2.5 \pm 0.3$ \\
\hline $2.8 \pm 0.5$ & $2.8 \pm 0.4$ & $2.9 \pm 0.5$ \\
\hline $2.9 \pm 0.6$ & $2.8 \pm 0.6$ & $2.9 \pm 0.6$ \\
\hline $8.2 \pm 0.9$ & $8.1 \pm 0.9$ & $8.3 \pm 0.8$ \\
\hline $296.8 \pm 79.3$ & $285.6 \pm 90.1$ & $297.0 \pm 74.7$ \\
\hline $368.8 \pm 103.7^{* *}$ & $351.6 \pm 116.9^{*}$ & $369.0 \pm 103.0$ \\
\hline $479.9 \pm 82.1^{* *}$ & $500.6 \pm 88.4 * *$ & $487.9 \pm 80.3^{* *}$ \\
\hline $294.0 \pm 63.1$ & $291.5 \pm 60.7$ & $284.6 \pm 67.3$ \\
\hline $24.8 \pm 11.4^{*}$ & $21.3 \pm 11.6$ & $24.2 \pm 11.0$ \\
\hline $6.3 \pm 7.3$ & $6.0 \pm 7.0$ & $7.9 \pm 8.2$ \\
\hline $31.0 \pm 16.7^{*}$ & $27.4 \pm 16.3$ & $32.1 \pm 15.9$ \\
\hline
\end{tabular}


TABLE 4.2B: Anthropometric characteristics, body composition, blood pressure, and parameters of glucose metabolism, lipids, liver function and inflammation at baseline and after one year of intervention $(\mathbf{n}=83)$

\begin{tabular}{|c|c|c|c|}
\hline & \multicolumn{3}{|c|}{ Baseline $(n=83)$} \\
\hline & $\frac{\text { Whole group }}{(n=83)}$ & $\frac{\mathrm{HP}}{(n=44)}$ & $\frac{\mathrm{MP}}{(n=39)}$ \\
\hline Outcome & mean $\pm S D$ & mean $\pm S D$ & mean $\pm S D$ \\
\hline \multicolumn{4}{|l|}{ General characteristics } \\
\hline \multicolumn{4}{|l|}{ Girls n(\%) } \\
\hline Age (yr) & $13.3 \pm 2.2$ & $13.7 \pm 2.4$ & $13.5 \pm 2.0$ \\
\hline Tanner stage & $3(2-4)$ & $3(2-5)$ & $3(2-4)$ \\
\hline \multicolumn{4}{|c|}{ Anthropometric characteristics } \\
\hline Height $(m)$ & $1.61 \pm 0.11$ & $1.61 \pm 0.10$ & $1.60 \pm 0.1$ \\
\hline Weight $(\mathrm{kg})$ & $77.5 \pm 20.3$ & $80.0 \pm 20.9$ & $75.7 \pm 18.2$ \\
\hline $\mathrm{BMI}(\mathrm{kg} / \mathrm{m} 2)$ & $29.4 \pm 4.9$ & $30.2 \pm 5.1$ & $29.3 \pm 4.6$ \\
\hline BMI z-score (SD) & $3.04 \pm 0.66$ & $3.10 \pm 0.69$ & $2.97 \pm 0.62$ \\
\hline Fat free mass $(\mathrm{kg})$ & $47.5 \pm 11.6$ & $47.5 \pm 12.3$ & $46.2 \pm 10.5$ \\
\hline Fat mass $(\mathrm{kg})$ & $31.0 \pm 11.8$ & $32.4 \pm 12.3$ & $29.3 \pm 11.0$ \\
\hline Fat mass (\%) & $38.8 \pm 8.7$ & $40.0 \pm 7.4$ & $38.2 \pm 8.9$ \\
\hline \multicolumn{4}{|l|}{ Blood pressure } \\
\hline $\mathrm{SBP}(\mathrm{mmHg})$ & $116.8 \pm 12.6$ & $116.8 \pm 12.9$ & $116.0 \pm 11.9$ \\
\hline $\mathrm{DBP}(\mathrm{mmHg})$ & $65.9 \pm 8.4$ & $67.3 \pm 8.0$ & $65.7 \pm 7.1$ \\
\hline $\mathrm{HR}(\mathrm{bpm})$ & $76.3 \pm 12.3$ & $76.1 \pm 13.3$ & $74.4 \pm 9.5$ \\
\hline \multicolumn{4}{|l|}{ Laboratory parameters } \\
\hline Glucose (mmol/L) & $4.5 \pm 0.7$ & $4.6 \pm 0.7$ & $4.5 \pm 0.7$ \\
\hline Insulin (pmol/L) & $114.3 \pm 87.6$ & $107.2 \pm 51.5$ & $112.4 \pm 94.6$ \\
\hline HOMA-IR & $3.57 \pm 2.62$ & $3.44 \pm 1.66$ & $3.52 \pm 2.87$ \\
\hline $\mathrm{HbA1c}(\mathrm{mmol} / \mathrm{mol})$ & $32.6 \pm 2.6$ & $32.7 \pm 2.9$ & $32.8 \pm 2.5$ \\
\hline C-peptide (nmol/L) & $0.9 \pm 0.3$ & $0.9 \pm 0.3$ & $0.9 \pm 0.3$ \\
\hline Total cholesterol (mmol/L) & $4.0 \pm 0.7$ & $4.1 \pm 0.7$ & $4.2 \pm 0.8$ \\
\hline HDL cholesterol (mmol/L) & $1.2 \pm 0.3$ & $1.3 \pm 0.3$ & $1.3 \pm 0.3$ \\
\hline LDL cholesterol (mmol/L) & $2.3 \pm 0.6$ & $2.4 \pm 0.6$ & $2.4 \pm 0.7$ \\
\hline $\mathrm{TG}(\mathrm{mmol} / \mathrm{L})$ & $1.1 \pm 0.5$ & $1.1 \pm 0.5$ & $1.1 \pm 0.6$ \\
\hline CRP $(\mathrm{mg} / \mathrm{L})$ & $2.9 \pm 3.1$ & $2.9 \pm 2.7$ & $3.0 \pm 3.5$ \\
\hline AST $(U / L)$ & $25.2 \pm 8.6$ & $23.9 \pm 7.4$ & $26.6 \pm 8.8$ \\
\hline ALT (U/L) & $25.5 \pm 17.4$ & $23.5 \pm 13.8$ & $26.2 \pm 15.5$ \\
\hline
\end{tabular}

HP: High-Protein Low-Glycaemic Index; MP: Medium-Protein Medium-Glycaemic Index; BMI = Body Mass Index; FFM = fat free mass; $F M=$ fat mass; $S B P=$ systolic blood pressure; $D B P=$ diastolic blood pressure; $H R=$ heart rate; $H O M A-I R=$ Homeostatic Model Assessment of Insulin Resistance (glucose ( $m$ mol/L)/insulin $(\mathrm{mU} / \mathrm{L}) * 22,5^{34} ; \mathrm{HDL}$ cholesterol=high-density lipoprotein cholesterol; $L D L$ cholesterol = low-density lipoprotein cholesterol; TG = triacylglycerides; $C R P=C$-reactive protein; $A S T$ = aspartate aminotransferase; $A L T=$ alanine aminotransferase; $E n \%=$ percentage of total energy intake; TFEQ = Three Factor Eating Questionnaire ${ }^{35} ; \mathrm{cpd}=$ counts per day; $c$ m = counts per minute; $P A=$ Physical Activity. Estimated effect changes are based upon imputated datasets. $P$-values are based upon comparison with baseline values. ${ }^{*} p<0.05$; ${ }^{* *} p<0.01$. 


\begin{tabular}{|c|c|c|}
\hline \multicolumn{3}{|c|}{$1 y$ follow-up } \\
\hline Whole group & $\underline{\mathrm{HP}}$ & $\underline{\mathrm{MP}}$ \\
\hline$(n=83)$ & $(n=44)$ & $(\overline{n=39})$ \\
\hline mean $\pm S D$ & mean $\pm S D$ & mean $\pm S D$ \\
\hline $14.5 \pm 2.2^{* *}$ & $14.7 \pm 2.5^{* *}$ & $14.3 \pm 1.9$ \\
\hline $4(3-5)^{* *}$ & $4(3-5)^{* *}$ & $3(3-4)$ \\
\hline $1.66 \pm 0.10^{* *}$ & $1.67 \pm 0.10^{* *}$ & $1.65 \pm 0.10$ \\
\hline $82.8 \pm 20.9^{* *}$ & $83.7 \pm 21.9^{* *}$ & $81.8 \pm 20.0$ \\
\hline $29.7 \pm 5.7$ & $29.6 \pm 5.9$ & $29.9 \pm 5.6$ \\
\hline $2.87 \pm 0.81^{* *}$ & $2.82 \pm 0.86^{* *}$ & $2.92 \pm 0.75$ \\
\hline $51.0 \pm 11.9^{* *}$ & $52.8 \pm 12.8^{* *}$ & $48.7 \pm 10.6$ \\
\hline $32.7 \pm 13.4^{*}$ & $34.0 \pm 14.1$ & $31.2 \pm 12.6$ \\
\hline $38.2 \pm 9.0$ & $38.4 \pm 8.8$ & $38.1 \pm 9.5$ \\
\hline $117.8 \pm 11.2$ & $118.1 \pm 12.7$ & $117.5 \pm 9.3$ \\
\hline $65.8 \pm 7.9$ & $67.1 \pm 8.4$ & $64.3 \pm 7.1$ \\
\hline $73.6 \pm 10.2^{*}$ & $73.7 \pm 11.8^{*}$ & $73.5 \pm 8.0$ \\
\hline $4.7 \pm 0.7^{*}$ & $4.8 \pm 0.7$ & $4.6 \pm 0.7$ \\
\hline $106.2 \pm 74.7$ & $95.4 \pm 60.5$ & $119.4 \pm 88.3$ \\
\hline $3.70 \pm 2.62$ & $3.45 \pm 2.42$ & $4.01 \pm 2.85$ \\
\hline $33.1 \pm 3.2$ & $32.8 \pm 3.5$ & $0.8 \pm 2.8$ \\
\hline $0.8 \pm 0.3$ & $0.8 \pm 0.3$ & $3.9 \pm 0.4$ \\
\hline $4.0 \pm 0.7$ & $4.2 \pm 0.7$ & $1.3 \pm 0.7$ \\
\hline $1.3 \pm 0.3$ & $1.3 \pm 0.3$ & $2.3 \pm 0.3$ \\
\hline $2.3 \pm 0.6$ & $2.3 \pm 0.6$ & $0.9 \pm 0.6$ \\
\hline $1.0 \pm 0.5$ & $1.1 \pm 0.6$ & $1.9 \pm 0.5$ \\
\hline $2.4 \pm 3.0$ & $2.7 \pm 3.7$ & $1.9 \pm 1.6$ \\
\hline $27.4 \pm 23.1$ & $23.3 \pm 6.4$ & $32.8 \pm 34.1$ \\
\hline $28.0 \pm 19.4$ & $28.4 \pm 20.4^{*}$ & $27.6 \pm 18.3$ \\
\hline
\end{tabular}


TABLE 4.3: Post-hoc associations of $\triangle B M I$ z-score and $\triangle H O M A-I R$ with lifestyle parameters $(n=126)$.

\begin{tabular}{|c|c|c|c|c|c|c|c|c|}
\hline & & $\Delta \mathbf{G I}$ & $\Delta \mathbf{G L}$ & $\begin{array}{l}\Delta \text { Energy } \\
\text { intake } \\
\text { (kJ) }\end{array}$ & $\begin{array}{l}\Delta \text { Protein } \\
\text { (En\%) }\end{array}$ & $\begin{array}{l}\Delta \text { TFEQ } \\
\text { F1 }\end{array}$ & $\begin{array}{l}\Delta \text { TFEQ } \\
\text { F2 }\end{array}$ & $\begin{array}{l}\Delta \text { TFEQ } \\
\text { F3 }\end{array}$ \\
\hline \multirow[t]{2}{*}{$\Delta$ BMI z-score $^{\neq}$} & $B(95 \% C l)$ & $\begin{array}{l}0.001 \\
(-0.013 ; \\
0.015)\end{array}$ & $\begin{array}{l}0.002 \\
(-0.001 ; \\
0.005)\end{array}$ & $\begin{array}{l}0.000 \\
(0.000 \\
0.000)\end{array}$ & $\begin{array}{l}-0.026 \\
(-0.060 ; \\
0.007)\end{array}$ & $\begin{array}{l}-0.027 \\
(-0.054 ; \\
-0.001)\end{array}$ & $\begin{array}{l}0.034 \\
(0.000 \\
0.068)\end{array}$ & $\begin{array}{l}0.034 \\
(0.008 ; \\
0.060)\end{array}$ \\
\hline & $p$-value & 0.891 & 0.279 & $0.010^{*}$ & 0.118 & $0.045^{*}$ & 0.052 & $0.013^{*}$ \\
\hline \multirow[t]{2}{*}{$\Delta$ HOMA-IR $^{\text {s }}$} & $B(95 \% C l)$ & $\begin{array}{l}0.134 \\
(0.014 ; \\
0.254)\end{array}$ & $\begin{array}{l}0.014 \\
(-0.014 ; \\
0.042)\end{array}$ & $\begin{array}{l}0.000 \\
(-0.001 ; \\
0.001)\end{array}$ & $\begin{array}{l}-0.167 \\
(-0.481 ; \\
0.147)\end{array}$ & $\begin{array}{l}-0.144 \\
(-0.363 ; \\
0.074)\end{array}$ & $\begin{array}{l}0.138 \\
(-0.128 ; \\
0.403)\end{array}$ & $\begin{array}{l}0.034 \\
(-0.190 \\
0.258)\end{array}$ \\
\hline & $p$-value & $0.031^{*}$ & 0.306 & 0.963 & 0.277 & 0.189 & 0.299 & 0.759 \\
\hline
\end{tabular}

Post-hoc associations of change in BMI z-score and HOMA-IR with changes in food intake. En\% = energy percentage; TFEQ = Three Factor Eating Questionnaire ${ }^{35}$. Associations are based upon the pooled imputed dataset. ^ Corrected for gender, age, Tanner $G / M$ stage at baseline and $\triangle$ Tanner G/M stage. ${ }^{*}$ Corrected for gender, age, Tanner G/M stage at baseline and $\Delta$ Tanner stage. ${ }^{*} p<0.05$; ${ }^{* *} p<0.01$

\section{Scatterplot for changes of BMI z-score with changes in HOMA-IR after one year of PREVIEW intervention}

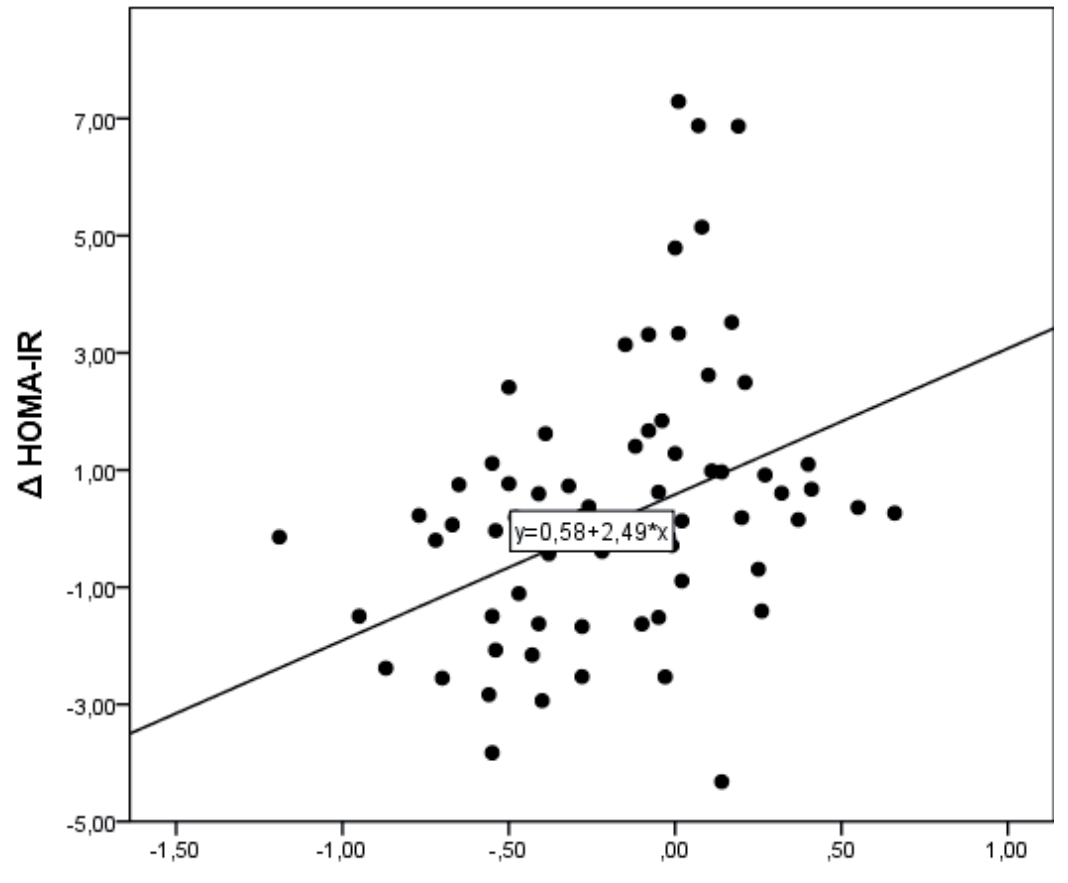

$\Delta$ BMI z-score

$\beta=2.234(2.070 ; 2.399), p<0.001^{* *}$

Figure 4.2: Association of change in BMI z-score with change in HOMA-IR. 


\section{DISCUSSION}

This PREVIEW study aimed to assess the effects of a high-protein low-GI vs. a mediumprotein medium-Gl diet on insulin resistance in adolescents with overweight and obesity and insulin resistance. No significant differences were found in reported protein intake and $\mathrm{Gl}$ between the two intervention groups, despite groups receiving different dietary instructions. No significant differences were observed between the two intervention groups regarding IR, parameters of glucose metabolism, lipid metabolism, inflammation, liver enzymes, anthropometric characteristics, or lifestyle factors at any timepoint. We conclude that the study as it was designed was not feasible.

Lack of feasibility was due to poor retention rates and lack of dietary compliance. Retention rates were $66 \%$ after $1 y$ and $39 \%$ after $2 y$. The HP group did not achieve the protein target of $25 \mathrm{En} \%$ and reported protein intake was not significantly different between the two intervention groups. One possible explanation might be that the protein intake estimates were based upon self-reported food diaries, which are known to be underreported ${ }^{39}$. Reported energy intake was 32.6-62.1\% lower than the energy requirements according to the WHO formula for adolescents of corresponding ages and weights ${ }^{29}$. The unmet protein target might partly be explained by reduced reward mechanisms in the brain or costs of high-protein foods ${ }^{40}$. Previous studies aiming to increase relative protein intake in adolescents also reported difficulties in dietary compliance ${ }^{11,13-18}$. Only half of them observed a difference in protein En\% between the higher-protein and the control group, which was often lower than the targets set between 22.5 and $25.0 \mathrm{En} \%{ }^{14,16,18}$. None of the studies observed a difference in BMI z-score decrease between intervention groups. The results from this study imply that achieving and maintaining a high-protein low-Gl diet during $1 \mathrm{y}$ or $2 \mathrm{y}$ is not feasible with instructions alone. Achieving and maintaining an energy target of $25 \mathrm{En} \%$ protein might only be feasible with vouchers/subsidies for foods high in protein, the use of protein supplements or meal replacements.

Post-hoc observations showed that in the complete group of adolescents with overweight/obesity and increased IR, a significant decrease in BMI z-score was observed while dietary restraint and MVPA were increased after $1 \mathrm{y}$ and $2 \mathrm{y}$. The reduction in BMI z-score was inversely related to change in dietary restraint and positively to change in hunger susceptibility and HOMA-IR change.

Furthermore, HOMA-IR stabilized after 1y of lifestyle intervention, despite progression in pubertal stage, but increased after $2 y$. As described previously, transient pubertal IR typically nadirs at mid-puberty, which might explain the increase of HOMA-IR after $2 y$ of study participation even though BMI z-score decreased ${ }^{5,6}$. Change in HOMA-IR at $1 y$ was positively related to changes in BMI z-score after correcting for relevant confounders. Half of the previously performed studies where reported protein intake did increase significantly observed a reduction in HOMA-IR in adolescents with an increased-protein diet ${ }^{11,12,14}$. 
However, these studies did not take pubertal stage into account. None of the studies found a significant effect of change in GI on anthropometric or cardiometabolic outcomes.

After both 1y and 2 yage-and sex-corrected BMIz-score was significantly reduced in these adolescents at high risk for T2DM development. After correcting for relevant confounders change in BMI z-score at 1y was negatively related to change in cognitive restraint of the TFEQ, which was increased significantly, and positively to change in susceptibility to hunger. Change in HOMA-IR at 1y was positively associated with change in BMI z-score independent of change in Tanner stage. This reduction of age- and sex-corrected BMI z-score of 0.17 SD is considered to be of clinical relevance, since a BMI z-score reduction of $\geq 0.15$ has been associated with significant increases in insulin sensitivity, decrease of total cholesterol and LDL-cholesterol concentrations, and normalization of blood pressure ${ }^{41,42}$.

TFEQ cognitive restraint scores were significantly higher after dietary instructions. Previous studies have shown that adolescents that have dieted in the past showed higher cognitive restraint and disinhibition scores on the TFEQ, compared to children without a history of dieting ${ }^{43}$. Moreover, a study in a similar cohort of Dutch adolescents showed an increase in dietary restraint scores during adolescence, especially in those with overweight/ obesity, indicating an increase in awareness of food intake and body-weight ${ }^{44,45}$. The observed reduction in BMI z-score was related to increased cognitive restraint scores, possibly as a result of more conscious eating behaviour after the dietary guidance, while supported by the positive relationship of change in BMI z-score with change in hunger susceptibility.

After $1 y$ and $2 y$ of personalized dietary and PA instructions, an increase in PA counts and moderate and vigorous intensity PA was observed, while sedentary behaviour decreased at all timepoints in both the completer as ITT-analyses, yet these observations were not associated with changes in HOMA-IR or BMI z-score.

By including all adolescents at increased risk of T2DM, regardless of age or specific obesity status, the study design was placed in a real life setting. Thus, the results of the PREVIEW study are representative of results that can be expected in out-centre treatments of all adolescents with overweight and obesity. Limitations were the use of HOMA-IR as a proxy of IR, and absence of an untreated control group due to ethical considerations of performing research in adolescents. In addition, no qualitative data was required on why participants did not meet the protein target and costs of diets was not considered in this study.

In conclusion, the PREVIEW study observed no effect of a high-protein low-GI diet on insulin resistance in adolescents with overweight/obesity and insulin resistance because of lack of feasibility. Post-hoc, attenuating pubertal insulin resistance was associated with BMI z-score decrease. BMI z-score decrease was associated with increased dietary restraint. 


\section{REFERENCES}

1. Lobstein T, Jackson-Leach R. Planning for the worst: estimates of obesity and comorbidities in school-age children in 2025. Pediatric obesity. 2016;11(5):321-325.

2. Olds T, Maher C, Zumin S, et al. Evidence that the prevalence of childhood overweight is plateauing: data from nine countries. International Journal of Pediatric Obesity. 2011;6(5-6):342360.

3. Skinner AC, Skelton JA. Prevalence and trends in obesity and severe obesity among children in the United States, 1999-2012. JAMA Pediatr. 2014;168(6):561-566.

4. Chiavaroli V, Giannini C, D'Adamo E, et al. Weight loss in obese prepubertal children: the influence of insulin resistance. Endocrine research. 2013;38(1):48-57.

5. Cree-Green M, Triolo TM, Nadeau KJ. Etiology of insulin resistance in youth with type 2 diabetes. Current diabetes reports. 2013;13(1):81-88.

6. Dorenbos E, Rijks JM, Adam TC, Westerterp-Plantenga MS, Vreugdenhil AC. Sleep efficiency as a determinant of insulin sensitivity in overweight and obese adolescents. Diabetes, obesity \& metabolism. 2015;17 Suppl 1:90-98.

7. Lee JM. Insulin resistance in children and adolescents. Reviews in endocrine \& metabolic disorders. 2006;7(3):141-147.

8. Levy-Marchal C, Arslanian S, Cutfield W, et al. Insulin resistance in children: consensus, perspective, and future directions. The Journal of clinical endocrinology and metabolism. 2010;95(12):5189-5198.

9. Xu L, Li M, Yin J, et al. Change of Body Composition and Adipokines and Their Relationship with Insulin Resistance across Pubertal Development in Obese and Nonobese Chinese Children: The BCAMS Study. International journal of endocrinology. 2012;2012:389108.

10. Skinner AC, Perrin EM, Moss LA, Skelton JA. Cardiometabolic Risks and Severity of Obesity in Children and Young Adults. The New England journal of medicine. 2015;373(14):1307-1317.

11. Baxter KA, Ware RS, Batch JA, Truby H. Predicting success: factors associated with weight change in obese youth undertaking a weight management program. Obesity research \& clinical practice. 2013;7(2):e147-e154.

12. Damsgaard CT, Papadaki A, Jensen SM, et al. Higher protein diets consumed ad libitum improve cardiovascular risk markers in children of overweight parents from eight European countries. The Journal of nutrition. 2013;143(6):810-817.

13. Duckworth LC, Gately PJ, Radley D, Cooke CB, King RF, Hill AJ. RCT of a high-protein diet on hunger motivation and weight-loss in obese children: an extension and replication. Obesity (Silver Spring, Md). 2009;17(9):1808-1810.

14. Garnett SP, Gow M, Ho M, et al. Improved insulin sensitivity and body composition, irrespective of macronutrient intake, after a 12 month intervention in adolescents with pre-diabetes; RESIST a randomised control trial. BMC Pediatr. 2014;14:289.

15. Gately PJ, King NA, Greatwood HC, et al. Does a high-protein diet improve weight loss in overweight and obese children? Obesity (Silver Spring, Md). 2007;15(6):1527-1534.

16. Mirza NM, Palmer MG, Sinclair KB, et al. Effects of a low glycemic load or a low-fat dietary intervention on body weight in obese Hispanic American children and adolescents: a randomized controlled trial. The American journal of clinical nutrition. 2013;97(2):276-285.

17. Papadaki A, Linardakis M, Larsen TM, et al. The effect of protein and glycemic index on children's body composition: the DiOGenes randomized study. Pediatrics. 2010;126(5):e1143-1152.

18. Rolland-Cachera MF, Thibault $\mathrm{H}$, Souberbielle JC, et al. Massive obesity in adolescents: dietary interventions and behaviours associated with weight regain at 2 y follow-up. International journal of obesity and related metabolic disorders : journal of the International Association for the Study of Obesity. 2004;28(4):514-519.

19. Noakes M. The role of protein in weight management. Asia Pac J Clin Nutr. 2008;17 Suppl 1:169171. 
20. Ludwig DS, Majzoub JA, Al-Zahrani A, Dallal GE, Blanco I, Roberts SB. High glycemic index foods, overeating, and obesity. Pediatrics. 1999;103(3):E26.

21. Bell LM, Watts K, Siafarikas A, et al. Exercise alone reduces insulin resistance in obese children independently of changes in body composition. The Journal of clinical endocrinology and metabolism. 2007;92(11):4230-4235.

22. Ho M, Garnett SP, Baur LA, et al. Impact of dietary and exercise interventions on weight change and metabolic outcomes in obese children and adolescents: a systematic review and metaanalysis of randomized trials. JAMA Pediatr. 2013;167(8):759-768.

23. Shaibi GQ, Cruz ML, Ball GD, et al. Effects of resistance training on insulin sensitivity in overweight Latino adolescent males. Med Sci Sports Exerc. 2006;38(7):1208-1215.

24. Shaw M, Savoye M, Cali A, Dziura J, Tamborlane WV, Caprio S. Effect of a successful intensive lifestyle program on insulin sensitivity and glucose tolerance in obese youth. Diabetes care. 2009;32(1):45-47.

25. Savoye M, Shaw M, Dziura J, et al. Effects of a weight management program on body composition and metabolic parameters in overweight children: a randomized controlled trial. Jama. 2007;297(24):2697-2704.

26. Savoye M, Caprio S, Dziura J, et al. Reversal of early abnormalities in glucose metabolism in obese youth: results of an intensive lifestyle randomized controlled trial. Diabetes care. 2014;37(2):317-324.

27. Hirst K, Baranowski T, DeBar L, et al. HEALTHY study rationale, design and methods: moderating risk of type 2 diabetes in multi-ethnic middle school students. International journal of obesity (2005). 2009;33 Suppl 4:S4-20.

28. Dorenbos E, Drummen M, Rijks J, et al. PREVIEW: Prevention of diabetes through lifestyle intervention in a multicentre study in Europe in children (10-17y). Design, methods, and baseline results. Diabetes, obesity \& metabolism. 2018;20(5):1096-1101.

29. Energy and protein requirements. Report of a joint FAO/WHO/UNU Expert Consultation. World Health Organ Tech Rep Ser. 2013;724:1-206.

30. de Onis M, Lobstein T. Defining obesity risk status in the general childhood population: which cut-offs should we use? Int J Pediatr Obes. 2010;5(6):458-460.

31. Lohman TG, Hingle M, Going SB. Body composition in children. Pediatr Exerc Sci. 2013;25(4):573590.

32. Marshall WA, Tanner JM. Variations in pattern of pubertal changes in girls. Arch Dis Child. 1969;44(235):291-303.

33. Marshall WA, Tanner JM. Variations in the pattern of pubertal changes in boys. Arch Dis Child. 1970;45(239):13-23.

34. Matthews DR, Hosker JP, Rudenski AS, Naylor BA, Treacher DF, Turner RC. Homeostasis model assessment: insulin resistance and beta-cell function from fasting plasma glucose and insulin concentrations in man. Diabetologia. 1985;28(7):412-419.

35. Stunkard AJ, Messick S. The three-factor eating questionnaire to measure dietary restraint, disinhibition and hunger. J Psychosom Res. 1985;29(1):71-83.

36. Baecke JA, Burema J, Frijters JE. A short questionnaire for the measurement of habitual physical activity in epidemiological studies. Am J Clin Nutr. 1982;36(5):936-942.

37. Rich C, Geraci M, Griffiths L, Sera F, Dezateux C, Cortina-Borja M. Quality Control Methods in Accelerometer Data Processing: Defining Minimum Wear Time. PLoS ONE. 2013;8(6):e67206.

38. Evenson KR, Catellier DJ, Gill K, Ondrak KS, McMurray RG. Calibration of two objective measures of physical activity for children. Journal of sports sciences. 2008;26(14):1557-1565.

39. Heitmann BL, Lissner L, Osler M. Do we eat less fat, or just report so? International journal of obesity and related metabolic disorders : journal of the International Association for the Study of Obesity. 2000;24(4):435-442. 
40. Born JM, Martens MJ, Lemmens SG, Goebel R, Westerterp-Plantenga MS. Protein v. carbohydrate intake differentially affects liking- and wanting-related brain signalling. The British journal of nutrition. 2013;109(2):376-381.

41. Kirk S, Zeller M, Claytor R, Santangelo M, Khoury PR, Daniels SR. The relationship of health outcomes to improvement in BMI in children and adolescents. Obesity research. 2005;13(5):876882.

42. O'Connor EA, Evans CV, Burda BU, Walsh ES, Eder M, Lozano P. Screening for Obesity and Intervention for Weight Management in Children and Adolescents: Evidence Report and Systematic Review for the US Preventive Services Task Force. Jama. 2017;317(23):2427-2444.

43. Gallant AR, Tremblay A, Perusse L, Despres JP, Bouchard C, Drapeau V. Past dieting is related to rigid control and disinhibition in adolescents from the Quebec Family Study. The British journal of nutrition. 2012;108(11):1976-1979.

44. Rutters F, Nieuwenhuizen AG, Vogels N, Bouwman F, Mariman E, Westerterp-Plantenga MS. Leptin-adiposity relationship changes, plus behavioral and parental factors, are involved in the development of body weight in a Dutch children cohort. Physiology \& behavior. 2008;93(45):967-974.

45. Nurkkala M, Kaikkonen K, Vanhala ML, Karhunen L, Keranen AM, Korpelainen R. Lifestyle intervention has a beneficial effect on eating behavior and long-term weight loss in obese adults. Eating behaviors. 2015;18:179-185. 


\title{
Chapter 5
}

Sleep duration is inversely related to BMI z-score in adolescents with overweight and obesity, independent of pubertal stage - a PREVIEW Study

\author{
Elke Dorenbos \\ Mathijs Drummen \\ Tanja Adam \\ Gareth Stratton \\ Nils Swindell \\ Anne Raben \\ Margriet Westerterp-Plantenga \\ Anita Vreugdenhil
}




\section{ABSTRACT}

\section{Background}

Inadequate sleep duration and quality are associated with increased risk of developing obesity, insulin resistance and cardiovascular risk in adolescents.

\section{Objectives}

To identify associations of sleep characteristics with anthropometric and cardiometabolic parameters and changes herein during the PREVIEW lifestyle intervention in adolescents with overweight/obesity.

\section{Methods}

67 adolescents (13.4 $\pm 2.2 \mathrm{y}$, BMI z-score 3.06 \pm 0.69$)$ received measurements for sleep architecture (using polysomnography), habitual sleep (using actigraphy) and self-reported sleep (PSQI questionnaire) at baseline and after one year of intervention. Sleep parameters were related to anthropometric and cardiometabolic characteristics.

\section{Results}

Baseline habitual total sleeping time (TST) was negatively associated with BMI z-score, corrected for Tanner stage and sex. N2 sleep duration was independently negatively associated with diastolic blood pressure. While BMI z-score decreased significantly after 1y, none of the sleep parameters had changed and were not related to outcome variables. PSQI (poor) sleep quality scores were inversely related to polysomnography-measured Quality Sleep, habitual TST, and positively with N2 sleep and Wake-After-Sleep-Onset duration. Habitual sleep duration and polysomnography-measured sleep duration were not related.

\section{Conclusions}

In adolescents with overweight/obesity habitual sleep duration was inversely related to BMI z-score, but not to cardiometabolic parameters. N2 sleep duration was negatively related to diastolic blood pressure. We recommend the combination of objective and self-reported sleep measurement methods. 


\section{INTRODUCTION}

As the prevalence of childhood obesity and obesity-related comorbidities increases, more research is performed to identify possible targets for obesity prevention and therapy'. Lifestyle interventions, mainly focussing on increasing physical activity and control of food intake, are currently the cornerstone of prevention and treatment of overweight and obesity-related comorbidities ${ }^{2}$. Recently more evidence is emerging that sleep may be a third modifiable contributor to energy balance, and consequently to obesity and related comorbidities. Identifying the relationship of sleep with obesity status might aid in optimizing treatment strategies and prevent development of morbidities for adolescents with overweight and obesity.

Puberty is associated with weight gain and a significant decline in sleep duration ${ }^{3-5}$. A growing body of evidence identified inadequate sleep duration and quality as an independent risk factor for weight gain in lean children, even after correcting for contributing factors such as BMI at the start of puberty or screentime ${ }^{3,6,7}$. In addition, decreased sleep duration and changes in sleep architecture, especially decrease of slow wave sleep (SWS), seem to be related to increased insulin resistance, hypertension, dyslipidaemia, and inflammatory factors ${ }^{6,8-11}$. Although the exact mechanism linking inadequate sleep with obesity and obesity-related comorbidities is not yet known, the most important mechanisms appear to be related to endocrine stress regulation. Sleep is a refractory period for stress hormones such as cortisol, norepinephrine and epinephrine. Loss of sleep or decrease of sleep quality may lead to increased endocrine stress. Experimental studies in children found that both increased cortisol concentrations and sympathetic nervous system activity were associated with unfavourable changes in glucose metabolism ${ }^{10}$. In addition, short sleep duration is associated with higher levels of the orexigenic hormone ghrelin and lower concentrations of the anorexigenic hormone leptin, which promotes hunger and food intake ${ }^{12}$. One experimental study found increased food intake after sleep restriction, and several observational studies reported increased intake of specifically high-energy and sugar rich foods ${ }^{11}$. Reduced sleep and subsequent daytime tiredness may also contribute to decreased PA and exercise in general ${ }^{8}$.

Although the majority of studies point to an inverse relation between sleep duration and the development of cardiometabolic risk, including obesity, evidence in children is still limited and often conflicting. Most studies have been performed in lean children or in general paediatric populations, but no research has yet been performed on the influence of sleep duration and architecture in children in whom overweight and obesity is already present. Also, the relationship between sleep characteristics and cardiometabolic risk parameters in adolescents has not yet been researched in longitudinal designs. Earlier studies were performed with different methods of sleep assessment e.g. polysomnography, 
actigraphy, self-reporting with questionnaires and/or parental sleep assessment, which limits the possibility for comparison of studies. Lastly, many studies did not correct for variables like pubertal stage, sex and obesity status, all of which are known to be related to sleep and cardiometabolic risk factors ${ }^{8}$.

The PREVIEW study in adolescents aimed to assess the effect of a lifestyle intervention on $\mathrm{BMI} z$-score and insulin resistance in adolescents with overweight/obesity ${ }^{13}$. The aim of the present study was to identify possible associations between sleep duration and architecture with anthropometric characteristics, parameters of glucose metabolism, cardiovascular risk, and inflammation in adolescents with overweight or obesity in a longitudinal design. In addition, this study aimed to compare the association between objective and subjective sleep assessment outcomes in adolescents with overweight and obesity. We hypothesized that sleep duration was negatively associated with BMI z-score and HOMA-IR, and that change in sleep duration would be inversely related to change in BMI z-score after one year.

\section{MATERIALS AND METHODS}

\section{Design and intervention}

The PREVIEW study in adolescents was a randomized controlled trial assessing the effects of increasing protein intake on obesity status and insulin resistance in adolescents with overweight/obesity and at increased risk of developing type 2 diabetes mellitus (T2DM), as described before ${ }^{18}$. Participants from three study sites (Maastricht University Medical Centre, the Netherlands; University of Navarra, Spain and Swansea University, United Kingdom) were instructed to increase dietary protein content and physical activity, and reduce glycaemic index of foods. At baseline and after one year of lifestyle intervention participants were subjected to regular measurements of anthropometric characteristics, blood sampling and questionnaires assessing lifestyle variables ${ }^{18}$. Adolescents recruited at Maastricht UMC were offered additional polysomnographies as part of their medical screening at baseline and after one year of study participation. Ethical approval was obtained from all sites and the trial was registered at Clinicaltrials.gov (NCT01777893).

\section{Participants}

Inclusion criteria for this study were an age between 10 and 17 years, overweight or (morbid) obesity defined as BMI z-score $\geq 1.0$ SD, increased risk of developing T2DM (defined as homeostatic model assessment of insulin resistance (HOMA-IR) $\geq 2.0$ for adolescents at Tanner stages $\geq 3$ or any HOMA-IR for adolescents at Tanner stages 1-2), availability of polysomnography data, and written informed consent from caregivers and adolescents aged $12 y$ or over $^{18}$. Exclusion criteria included diagnosis of medical conditions or use of medications that might compromise study outcomes (e.g. diabetes, bariatric surgery or 
metformin use), and issues that might limit study compliance (e.g. severe food intolerances). A total of 67 adolescents recruited at Maastricht University participated in this study for which additional polysomnographies were performed. Participant characteristics are presented in Table 5.1.

\section{Measurements}

\subsection{Anthropometric measurements, body composition and pubertal stage}

Height and weight were measured while adolescents were in fasted state, barefoot, and wearing only underwear, using a calibrated scale (Seca, Chino, CA, USA) and wall-based stadiometer (De Grood Metaaltechniek, Nijmegen, the Netherlands). Because body mass index (BMI) in childhood is affected by periods of accelerated growth, age- and sex-adjusted BMI z-scores were calculated (Growth Analyzer VE, Rotterdam, the Netherlands) and obesity status using international obesity cut-off points ${ }^{14}$. Body composition was assessed using air displacement plethysmography (BodPod, Life Measurement Instruments, Concord, CA, USA $)^{15}$. Pubertal stage was assessed according to the Tanner stadia for boys and girls ${ }^{16,17}$.

\subsection{Parameters of glucose metabolism, lipids, inflammation and blood pressure}

After an overnight fast, blood samples were taken to measure concentrations of fasting blood glucose, total cholesterol, high-density lipoprotein (HDL) cholesterol, low-density lipoprotein (LDL) cholesterol, and C-reactive protein (CRP, COBAS 800 modular analyser, Roche, Woerden, the Netherlands). Fasting insulin and $\mathrm{HbA1c}$ concentrations were measured with the fully automated HPLC Variant II 15 (Bio-Rad Laboratories, Veenendaal, the Netherlands). HOMA-IR (calculated as fasting glucose concentration (mmol/L) * fasting insulin concentration ( $\mathrm{mU} / \mathrm{L}$ )/22.5) was used as a proxy of insulin resistance ${ }^{18}$. Blood pressure and heart rate were measured three times on the right arm (Mobil-O-Graph, I.E.M., GmbH, Stolberg, Germany). An average of 3 measurements was used for analyses.

\subsection{Sleep}

Sleep was objectively measured using polysomnography during an overnight stay at the paediatric intensive care unit of Maastricht UMC. Analyses with BrainRT (v2.1, OSG, Rumst, Belgium) assessed duration of Total Sleeping Time (TST), Wake After Sleep Onset (WASO), and the different sleep stages Rapid Eye Movement (REM) sleep, and non-REM sleep phases N1, N2 and N3 (also known as Slow Wave Sleep (SWS)). In addition, Quality Sleep (QS, calculated as (REM+SWS)/TST) was determined ${ }^{19}$. Habitual TST was measured during 4 consecutive nights at home with the Actisleep GT3X (Actigraph Corp., Pensacola, FL, USA), applying a fully automated algorithm developed for use in 24-h waist worn accelerometer protocols. The algorithm produces estimates of a nocturnal sleep period that are compared with an expert visual inspection of accelerometer trace ${ }^{20}$. Subjectively experienced sleep 
was assessed using the Pittsburgh Sleep Quality Index (PSQI), where higher scores indicate poorer sleep quality and which for clarity will be formulated as PSQI (poor) sleep quality in this paper ${ }^{21}$.

\section{Statistical analyses}

A sample size of 40 adolescents at $1 y$ follow-up was required to demonstrate an association of changes in sleep duration with HOMA-IR with an alpha of 0.05 and a power of $0.80^{9}$. Analyses were performed with IBM SPSS Statistics for Windows (v24, IBM Corp., Armonk, NY, USA). Comparison of drop-outs with study completers were assessed with independent Student's T-tests and Mann-Whitney- $U$ tests, as appropriate. Changes over time were determined with repeated measures ANOVA or Wilcoxon signed rank analyses in the group that had a follow-up polysomnography assessment. Associations between (change in) sleep parameters and (change in) outcome parameters were assessed using Pearson's or Spearman's correlation coefficients, as appropriate. Associations at baseline were corrected for sex, Tanner stage and BMI z-score. A two-sided p-value of 0.05 or less was considered to be statistically significant.

\section{RESULTS}

Sixty-seven participants from PREVIEW were eligible for this sleep study (Figure 5.1). After one year of study participation, a second polysomnography was performed in 29 subjects (43.3\%). Reasons for not participating in the follow-up polysomnography were drop-out of the PREVIEW study $(n=18)$ or refusal of a second polysomnography measurement $(n=20)$. Baseline characteristics are presented in Table 5.1.

At baseline, actigraphy-measured habitual TST was negatively associated with age, BMI z-score, absolute fat mass, and fat mass as a percentage of body weight, and was associated with sex (Table 5.2). Polysomnography-measured SWS and QS were negatively related to age, Tanner stage, FFM and FM. Time spent in phase N2 sleep was positively related to age, Tanner stage and FFM. After correcting for Tanner stage and sex, only inverse associations of habitual TST with BMI z-score, FFM and FM remained significant. No associations were observed between the outcome of the sleep questionnaire and anthropometric characteristics. 


\section{7 eligible for baseline polysomnography analyses}

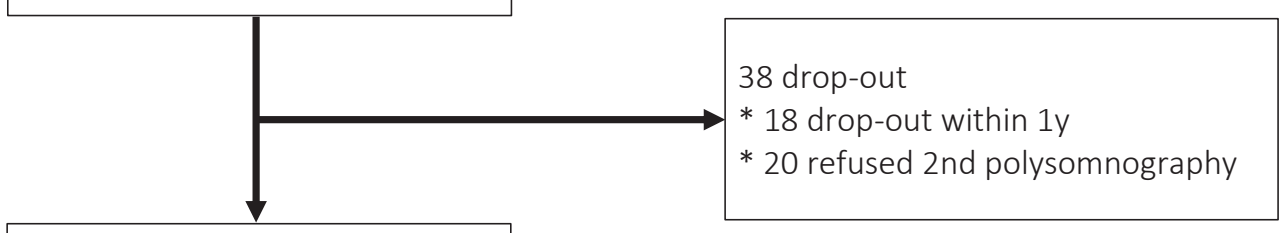

29 included for polysomnography follow-up analyses at $1 \mathrm{y}$

Figure 5.1: Flowchart

Associations of sleep parameters with glucose metabolism, cardiovascular risk and inflammation were corrected for Tanner stage, BMI z-score and sex (Table 5.2). Phase N2 sleep was inversely related to diastolic blood pressure, independent of the correction factors mentioned (Table 5.3). No other associations were found between sleep duration or sleep architecture parameters and glucose metabolism, cardiovascular risk, or inflammation.

Twenty-nine subjects (43.3\%) participated in the sleep assessment after one year. No significant differences in anthropometric characteristics, cardiovascular risk or sleep parameters were observed between the study completers and drop-outs (data not shown). The characteristics of the 29 adolescents participating in both baseline and follow-up polysomnography are presented in Table 5.1. After one year mean BMI z-score decreased significantly while height, weight, FFM and FM increased (Dorenbos et al. submitted). Changes were observed in neither sleep duration or sleep phases, nor in self-reported sleep quality using the PSQI questionnaire (Table 5.1).

Comparison of the different sleep assessment methods was made to identify possible associations between objective and subjective sleep characteristics (Table 5.4). No associations were found between habitual TST, measured by actigraphy at home, and polysomnography-measured TST assessed during hospital admission. PSG-measured Quality Sleep was negatively correlated with PSQI (poor) sleep quality, while phase N2 sleep was positively associated with PSQI (poor) sleep quality. WASO was positively related to daytime dysfunction on the PSQI questionnaire. Habitual TST, measured by actigraphy, was negatively correlated with PSQI (shorter) sleep duration. 


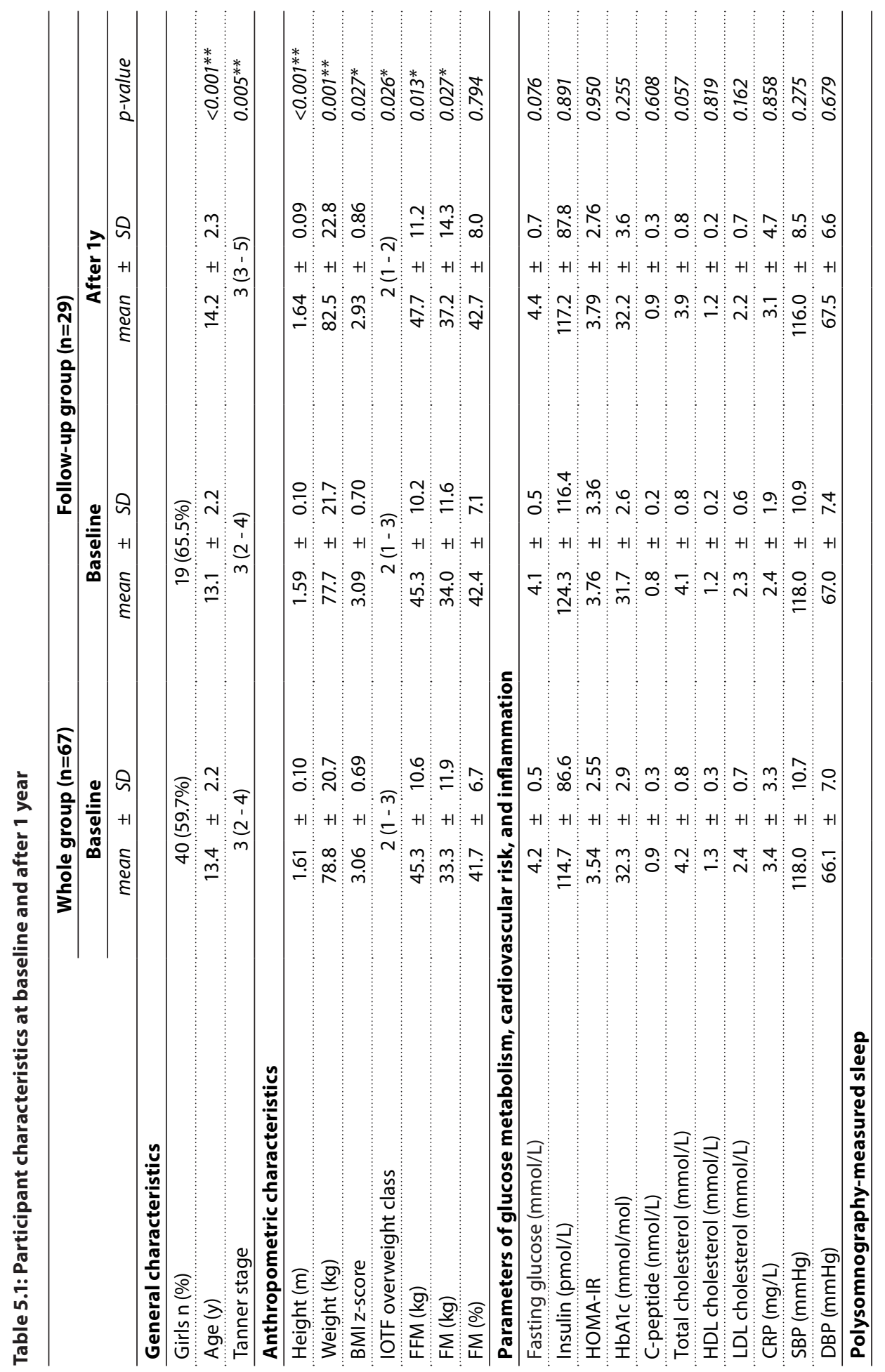




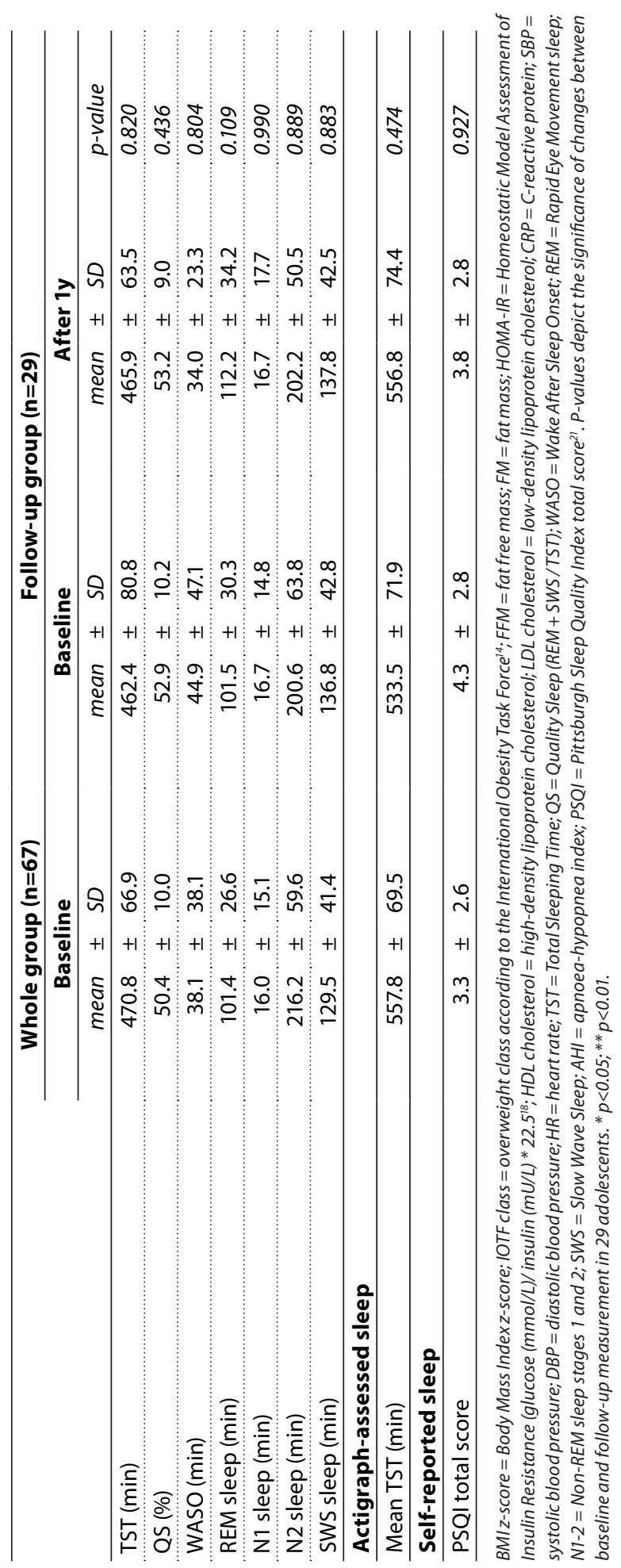




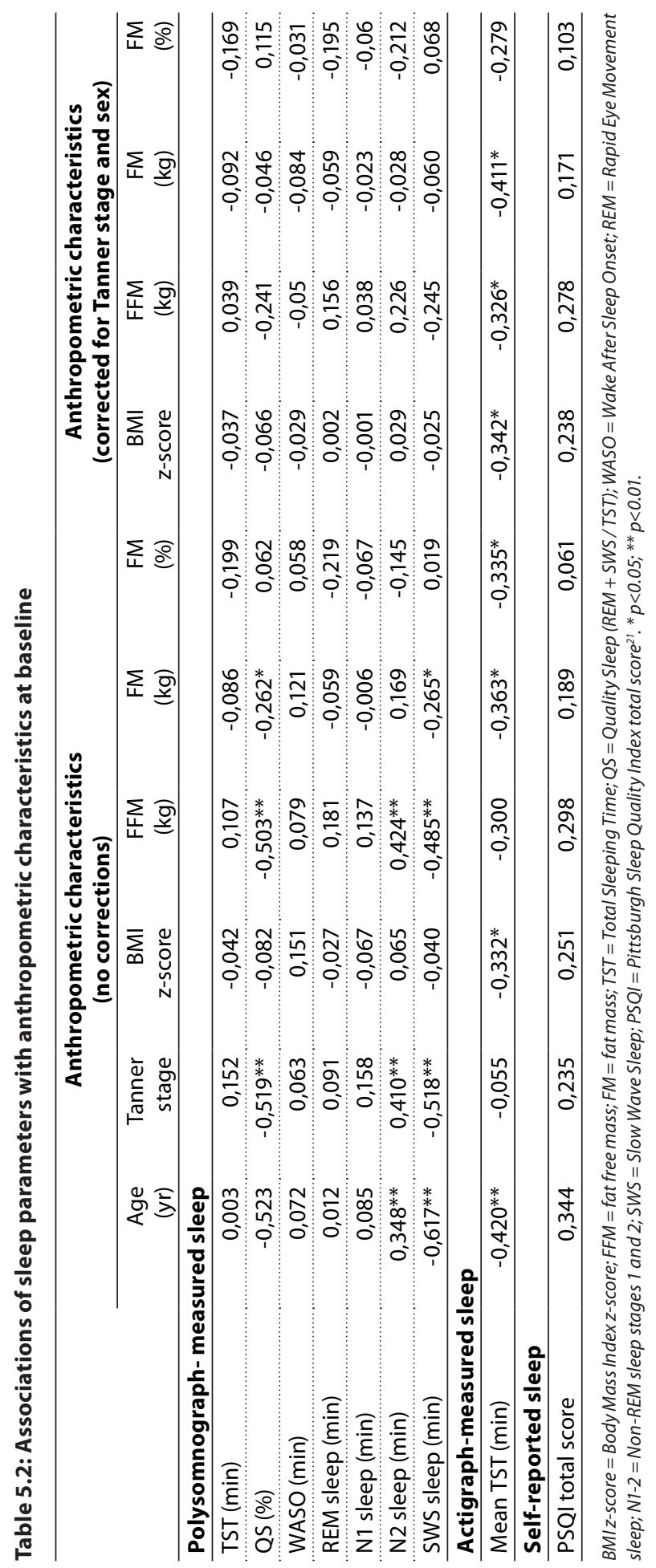




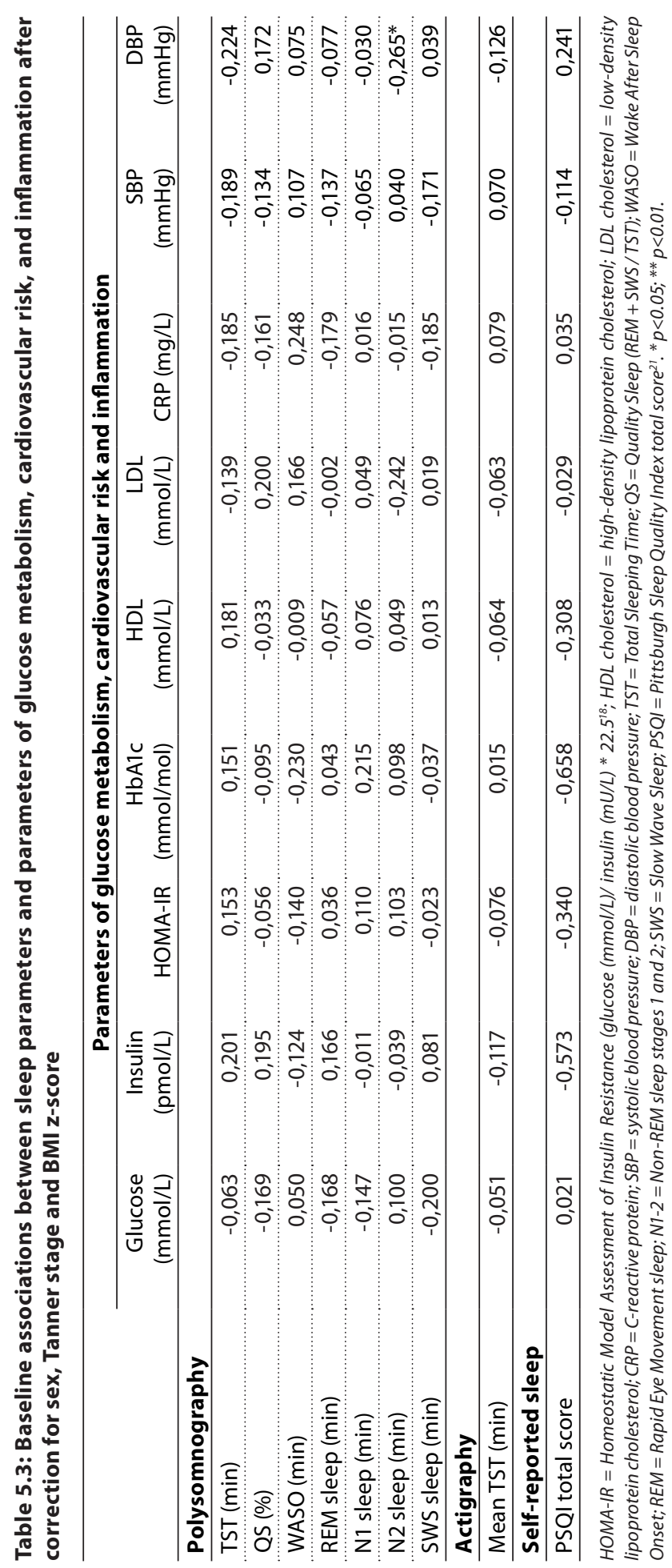




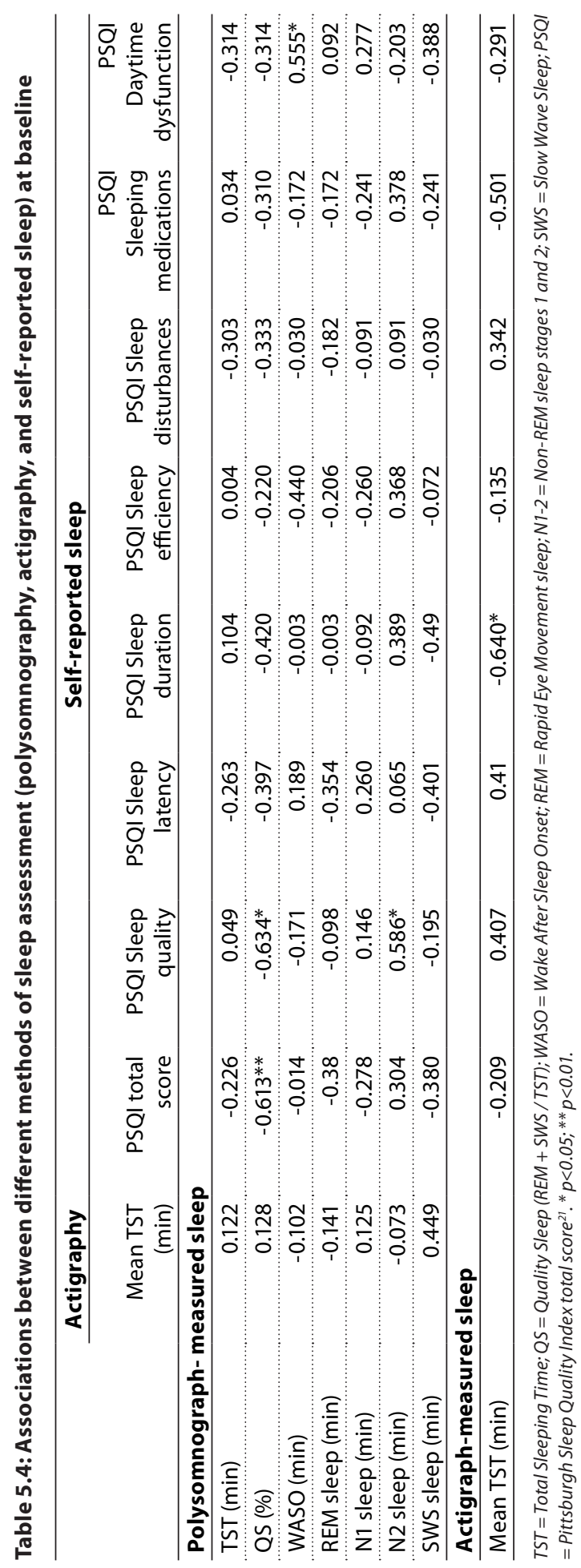




\section{DISCUSSION}

This is the first study to assess the relationship of objective and subjective sleep characteristics with anthropometric and cardiometabolic parameters and changes herein in adolescents with overweight and obesity. After correcting for pubertal stage and sex, habitual sleep duration was negatively related to BMI z-score. Phase N2 sleep was negatively related to diastolic blood pressure, independent of puberty, BMI z-score and sex, but no other sleep parameters were associated with anthropometry or cardiometabolic risk. PSQI (poor) sleep quality scores were inversely associated with PSG-measured Quality Sleep, habitual TST, and positively with phase N2 sleep and WASO (wake-up after sleep onset). Habitual sleep duration (measured by actigraphy) and polysomnography measured sleep durations were not related.

At baseline, the inverse relation between habitual TST with BMI z-score, absolute fat mass and fat free mass remained significant after correction for pubertal stage and sex, indicating that adolescents with overweight/obesity with a higher BMI z-score had shorter sleep duration, independent of pubertal stage. Earlier studies have already shown an inverse association between sleep duration and obesity in adolescents and adults of all weight classes ${ }^{6-8}$. These results suggest that in adolescents with overweight/obesity, sleep duration but not parameters of sleep architecture, were related to severity of overweight and body composition.

Associations between sleep variables and parameters of glucose metabolism, cardiovascular risk and inflammation were corrected for Tanner stage, BMI z-score and sex to eliminate confounding effects of obesity status and puberty. Phase N2 sleep duration was negatively related to diastolic blood pressure at baseline, suggesting that adolescents who had less phase N2 sleep had higher diastolic blood pressure. A weak negative association between sleep duration and DBP has been observed before, and is speculated to be related to nocturnal non-dipping in adolescents with obesity and increased sympathetic nervous system activity ${ }^{8,10,22}$. No associations were observed between total sleep duration, duration of the different sleep stages, and self-reported sleep, with parameters of insulin resistance, cardiovascular risk and inflammation after correction for confounders. A recent meta-analysis of studies found no overall association between sleep duration and HOMA$\mathrm{IR}^{8}$. Although some studies reported positive associations between SWS sleep duration and measures of insulin sensitivity ${ }^{23-25}$, those findings could not be confirmed in this study. It should however be noted that these studies were performed in mixed groups of lean and overweight adolescents, whereas in this cohort all adolescents were overweight/obese and had relatively high HOMA-IR concentrations at baseline. In line with our findings, studies in lean and obese adolescents observed no overall associations between sleep duration and phases with dyslipidaemia and $\mathrm{CRP}^{8,26}$. 
To this date, only one study in adolescents has been performed in a longitudinal design ${ }^{27}$. In this cohort 29 adolescents (43.3\%) participated in a second polysomnography. Drop-out or refusal to undergo a second polysomnography might be due to the intensive nature of the measurement (polysomnography required an overnight stay at the paediatric ward). None of the measured parameters of sleep duration and architecture at baseline were significantly different between adolescents that participated in a second polysomnography and drop-outs. Both objectively measured and self-reported sleep parameters did not change significantly during 1-year study participation. We speculate that the reduction in BMI z-score may have counteracted the reduction in sleep duration as was earlier reported by progressing age and Tanner stage ${ }^{3}$.

Polysomnography is the gold standard for assessment of sleep architecture but requires overnight hospital admission, making it an invasive and costly procedure. In adolescents mainly actigraphy to assess daily sleep during multiple nights, or sleep questionnaires to assess experienced sleep quality (PSQI) are used. In this study both objective and subjective sleep measurement methods were combined to assess a full range of objectively measured and experienced sleep parameters. Self-reported poor sleep duration was negatively associated with actigraph-measured TST, and higher self-assessed sleep quality was related to higher percentages of SWS and REM sleep. Similarly, higher WASO was positively related with higher self-reported daytime dysfunction scores. These relationships suggest that PSQI scores are indicative for polysomnography outcomes. On the other hand, associations of sleeping duration with anthropometric characteristics were different for actigraphmeasured TST and polysomnography-measured TST, and these two were not interrelated $(r=-0.112, p=0.090)$. This might be explained by measurements during different nights: actigraph-measured habitual TST was measured during 4 consecutive nights at home while polysomnography-TST was measured during one night at an in-hospital setting. In conclusion, PSQI (poor) sleep quality scores were indicative of polysomnography outcomes, while actigraphy and polysomnography measured sleep duration were not related ${ }^{28}$. The use of both field and self-assessed sleep measurement methods may complement each other when combined.

As far as we know, this is the first exploratory study to assess the relationship of sleep parameters with anthropometric characteristics and cardiometabolic parameters and changes herein over time, in adolescents with overweight and obesity. Moreover, this is the first study to use longitudinal PSG measurements and one of the first to use multiple actigraph-measurements over time in adolescents without sleep syndromes ${ }^{9,29-31}$. Strengths of this study are the longitudinal design where adolescents were measured during one year follow-up, as well as the correction for confounders puberty, sex and BMI z-score. Also novel is the combination of both objective and subjective sleep measures in an adolescent cohort. A limitation is the large drop-out for a second polysomnography measurement resulting in a small sample of adolescents eligible for longitudinal assessments. 
In conclusion, the inverse association of sleep duration with BMI z-score in adolescents with overweight/obesity confirms earlier observations of sleep duration with risk of obesity. Previously reported relationships with insulin resistance, cardiometabolic risk or inflammation were not confirmed in adolescents with overweight/obesity. Phase N2 sleep duration was inversely associated with diastolic blood pressure. PSQI (poor) sleep quality scores were indicative of polysomnography outcomes, while actigraph- and polysomnography-measured sleep duration were not related. More studies are needed to assess changes in sleep duration and architecture parameters, and the effects of sleep hygiene interventions, on cardiometabolic health in adolescents without sleep syndromes. We would recommend future sleep studies to include both objective as well as self-reported sleep measurement methods. 


\section{REFERENCES}

1. Lobstein T, Jackson-Leach R. Planning for the worst: estimates of obesity and comorbidities in school-age children in 2025. Pediatr Obes. 2016;11(5):321-325.

2. Oude Luttikhuis $\mathrm{H}$, Baur $\mathrm{L}$, Jansen $\mathrm{H}$, et al. Interventions for treating obesity in children. Cochrane Database Syst Rev. 2009(1):Cd001872.

3. Rutters F, Gerver WJ, Nieuwenhuizen AG, Verhoef SPM, Westerterp-Plantenga MS. Sleep duration and body-weight development during puberty in a Dutch children cohort. International Journal Of Obesity. 2010;34:1508.

4. Knutson KL. The association between pubertal status and sleep duration and quality among a nationally representative sample of U. S. adolescents. Am J Hum Biol. 2005;17(4):418-424.

5. Thorleifsdottir B, Bjornsson JK, Benediktsdottir B, Gislason T, Kristbjarnarson H. Sleep and sleep habits from childhood to young adulthood over a 10-year period. J Psychosom Res. 2002;53(1):529-537.

6. Cappuccio FP, Taggart FM, Kandala N-B, et al. Meta-Analysis of Short Sleep Duration and Obesity in Children and Adults. Sleep. 2008;31(5):619-626.

7. Fatima Y, Doi SAR, Mamun AA. Longitudinal impact of sleep on overweight and obesity in children and adolescents: a systematic review and bias-adjusted meta-analysis. Obesity Reviews. 2015;16(2):137-149.

8. Quist JS, Sjödin A, Chaput J-P, Hjorth MF. Sleep and cardiometabolic risk in children and adolescents. Sleep Medicine Reviews. 2016;29:76-100.

9. Klingenberg L, Chaput JP, Holmback U, et al. Acute Sleep Restriction Reduces Insulin Sensitivity in Adolescent Boys. Sleep. 2013;36(7):1085-1090.

10. Schmid SM, Hallschmid M, Schultes B. The metabolic burden of sleep loss. Lancet Diabetes Endocrinol. 2015;3(1):52-62.

11. Garaulet M, Ortega FB, Ruiz JR, et al. Short sleep duration is associated with increased obesity markers in European adolescents: effect of physical activity and dietary habits. The HELENA study. International journal of obesity (2005). 2011;35(10):1308-1317.

12. Hart CN, Carskadon MA, Considine RV, et al. Changes in Children's Sleep Duration on Food Intake, Weight, and Leptin. Pediatrics. 2013;132(6):e1473.

13. Dorenbos E, Drummen M, Rijks J, et al. PREVIEW (Prevention of Diabetes Through Lifestyle Intervention and Population Studies in Europe and Around the World) study in children aged 10 to 17 years: Design, methods and baseline results. Diabetes, obesity \& metabolism. 2018;20(5):1096-1101.

14. Cole TJ, Lobstein T. Extended international (IOTF) body mass index cut-offs for thinness, overweight and obesity. Pediatr Obes. 2012;7(4):284-294.

15. Lohman TG. Assessment of Body Composition in Children. Pediatric Exercise Science. 1989:1930.

16. Marshall WA, Tanner JM. Variations in pattern of pubertal changes in girls. Arch Dis Child. 1969;44(235):291-303.

17. Marshall WA, Tanner JM. Variations in the pattern of pubertal changes in boys. Arch Dis Child. 1970;45(239):13-23.

18. Matthews DR, Hosker JP, Rudenski AS, Naylor BA, Treacher DF, Turner RC. Homeostasis model assessment: insulin resistance and beta-cell function from fasting plasma glucose and insulin concentrations in man. Diabetologia. 1985;28(7):412-419.

19. Berry RB, Budhiraja R, Gottlieb DJ, et al. Rules for scoring respiratory events in sleep: update of the 2007 AASM Manual for the Scoring of Sleep and Associated Events. Deliberations of the Sleep Apnea Definitions Task Force of the American Academy of Sleep Medicine. J Clin Sleep Med. 2012;8(5):597-619. 
20. Tudor-Locke C, Barreira TV, Schuna JM, Jr., Mire EF, Katzmarzyk PT. Fully automated waistworn accelerometer algorithm for detecting children's sleep-period time separate from 24-h physical activity or sedentary behaviors. Applied physiology, nutrition, and metabolism = Physiologie appliquee, nutrition et metabolisme. 2014;39(1):53-57.

21. Buysse DJ, Reynolds CF, 3rd, Monk TH, Berman SR, Kupfer DJ. The Pittsburgh Sleep Quality Index: a new instrument for psychiatric practice and research. Psychiatry Res. 1989;28(2):193213.

22. Westerstahl M, Hedvall Kallerman P, Hagman E, Ek AE, Rossner SM, Marcus C. Nocturnal blood pressure non-dipping is prevalent in severely obese, prepubertal and early pubertal children. Acta Paediatr. 2014;103(2):225-230.

23. Koren D, Levitt Katz LE, Brar PC, Gallagher PR, Berkowitz RI, Brooks LJ. Sleep architecture and glucose and insulin homeostasis in obese adolescents. Diabetes Care. 2011;34(11):2442-2447.

24. Zhu Y, Li AM, Au CT, et al. Association between sleep architecture and glucose tolerance in children and adolescents. J Diabetes. 2015;7(1):10-15.

25. Armitage R, Lee J, Bertram H, Hoffmann R. A preliminary study of slow-wave EEG activity and insulin sensitivity in adolescents. Sleep Med. 2013;14(3):257-260.

26. Hjorth M, Chaput J-P, Damsgaard C, et al. Low Physical Activity Level and Short Sleep Duration Are Associated with an Increased Cardio-Metabolic Risk Profile: A Longitudinal Study in 8-11 Year Old Danish Children. Vol 92014.

27. Cespedes EM, Rifas-Shiman SL, Redline S, Gillman MW, Pena MM, Taveras EM. Longitudinal associations of sleep curtailment with metabolic risk in mid-childhood. Obesity (Silver Spring). 2014;22(12):2586-2592.

28. Buysse DJ, Hall ML, Strollo PJ, et al. Relationships between the Pittsburgh Sleep Quality Index (PSQI), Epworth Sleepiness Scale (ESS), and clinical/polysomnographic measures in a community sample. J Clin Sleep Med. 2008;4(6):563-571.

29. Chaput JP, Lambert M, Gray-Donald K, et al. Short sleep duration is independently associated with overweight and obesity in Quebec children. Canadian journal of public health $=$ Revue canadienne de sante publique. 2011;102(5):369-374.

30. Javaheri S, Storfer-Isser A, Rosen CL, Redline S. Association of short and long sleep durations with insulin sensitivity in adolescents. The Journal of pediatrics. 2011;158(4):617-623.

31. Martinez-Gomez D, Eisenmann JC, Gomez-Martinez S, et al. Sleep duration and emerging cardiometabolic risk markers in adolescents. The AFINOS study. Sleep Med. 2011;12(10):9971002. 


\section{Chapter 6}

\section{Role of aminotransferase concentration in insulin resistance and $\mathrm{BMI}$ z-score change in adolescents with overweight/ obesity during intervention - a PREVIEW study}

Elke Dorenbos

Mathijs Drummen

Tanja Adam

J. Alfredo Martínez

Santiago Navas-Carretero

Gareth Stratton
Nils Swindell

Pauline Stouthart Mikael Fogelholm Anne Raben Margriet Westerterp-Plantenga Anita Vreugdenhil 


\section{ABSTRACT}

\section{Introduction}

Non-alcoholic fatty liver disease (NAFLD) and insulin resistance (IR) often co-develop with obesity. This study assessed associations between NAFLD activity, IR and BMI z-score in adolescents with overweight/obesity during lifestyle intervention.

\section{Methods}

126 participants from the PREVIEW study (59\% girls, BMI z-score 3.04 \pm 0.66 ) were guided to increase protein intake and physical activity. Changes in BMI z-score and HOMA-IR were assessed in participants with aminotransferase (ALT) concentrations below and above the upper limit of normal (ULN), indicative of NAFLD.

\section{Results}

$32.5 \%$ of adolescents presented with ALT concentrations $>$ ULN. Change in BMI z-score was significantly less in subjects with ALT concentrations $>$ ULN after 1y intervention, compared to subjects with ALT concentration $<U L N$. NAFLD was positively related to BMI z-score and change herein after 1y. Baseline ALT concentration was positively associated with HOMA-IR.

\section{Conclusions}

Increased ALT concentration, indicative of NAFLD, was positively associated with baseline BMI z-score, and associated with less BMI z-score change after 1y lifestyle intervention. Baseline ALT concentration was positively related to IR. These associations should be confirmed in larger studies. 


\section{INTRODUCTION}

One of the major concerns of the obesity epidemic is the surge in obesity-related comorbidities in adolescents, such as insulin resistance (IR), dyslipidaemia and non-alcoholic fatty liver disease (NAFLD) 1 . IR has been shown to be a risk factor for developing type 2 diabetes mellitus, and previously we and others have shown IR to be related to BMI $z$-score in adolescents with overweight/obesity ${ }^{2-8}$. NAFLD encompasses a spectrum of liver diseases in the absence of excessive alcohol consumption, ranging from hepatic steatosis to steatohepatitis, liver fibrosis and cirrhosis, and has been related to obesity ${ }^{9,10}$. Measurement of serum aminotransferase (ALT) concentrations are commonly used as a proxy for NAFLD in children". Interestingly, IR and NAFLD frequently co-develop in adolescents with overweight/obesity and appear to affect each other negatively. It has been proposed that their pathophysiological mechanisms are largely similar ${ }^{9,10,12,13}$. Increased circulating free fatty acids (FFA) are related to ectopic lipid deposition in muscles and in the liver, thus contributing to liver steatosis, as well as promoting a low-grade inflammatory state $9,10,12,13$. Inflammation and elevated FFA concentrations reduce cellular glucose uptake, thereby inducing IR and a compensatory increase in pancreatic $\beta$-cell insulin secretion ${ }^{14}$. Thus, IR and NAFLD reinforce one another, where IR is a driver for the development of NAFLD by contributing to hepatic steatosis, and NAFLD might exacerbate especially hepatic $\mathbb{I R}^{9,10,12,13,15,16}$.

Recent studies demonstrated that adolescents who were insulin resistant were less successful in decreasing BMI z-score during intervention than adolescents that were not insulin resistant, suggesting that the presence of comorbidities might relate to outcomes of lifestyle interventions targeting adolescent obesity ${ }^{17,18}$. Considering the closely related pathophysiological mechanisms of IR and NAFLD, it is relevant to assess the effect and associations of NAFLD activity with BMI z-score, IR and intervention-induced changes thereof ${ }^{2,19}$.

The PREVIEW study in adolescents aimed to assess the effect of lifestyle intervention to decrease IR and BMI z-score in adolescents with overweight/obesity ${ }^{20}$. The purpose of this substudy was to assess the possible contribution of NAFLD, indicated by increased serum ALT concentrations, to BMI z-score, glucose metabolism and changes herein in adolescents with overweight/obesity during lifestyle intervention. 


\section{MATERIALS AND METHODS}

\section{Study design}

The PREVIEW study in adolescents was a lifestyle intervention designed to assess the role of lifestyle interventions on anthropometric characteristics and parameters of glucose metabolism and liver transaminases in adolescents with overweight/obesity and $\mathrm{IR}^{20}$. As previously published, participants received instructions to increase dietary protein intake while reducing glycaemic index, and to increase physical activity (PA) ${ }^{20}$. The study was approved by local Medical Ethics Committees, compliant with the Declaration of Helsinki and ICH-GCP and published on ClinicalTrials.gov (no. NCT01777893).

\section{Participants}

126 Adolescents were recruited at Maastricht University (Maastricht, the Netherlands), University of Navarra (Pamplona, Spain) and Swansea University (Swansea, United Kingdom). Inclusion criteria were overweight or obesity (defined as BMI z-score $>1.0 \mathrm{SD}$ ), increased IR (defined as Homeostatic Model Assessment of Insulin Resistance (HOMA-IR) >2.0 for adolescents Tanner stages $\geq 3$ or any HOMA-IR at Tanner stages 1-2) and signed informed consent from parents and adolescents $\geq 12 y$. Exclusion criteria included medical conditions or medication use that could influence study outcomes (e.g. bariatric surgery, T2DM, metformin use) ${ }^{20}$, or secondary causes of elevated ALT concentrations (e.g. viral hepatitis).

\section{Measurements}

Height and weight were measured using a wall-mounted stadiometer (De Grood Metaaltechniek, Nijmegen, the Netherlands) and digital scale (Seca, Chino, CA, USA) while adolescents were in a fasted state, barefoot, and wearing only underwear. Age- and sexcorrected BMI z-scores were calculated to assess overweight and obesity (TNO Growth Calculator, Den Haag, the Netherlands) ${ }^{21}$.

Blood samples were obtained after an overnight fast. Glucose and ALT concentrations were analysed using the COBAS 800 modular analyser (Roche, Woerden, the Netherlands). Concentrations of insulin and $\mathrm{HbA1c}$ were measured with the fully automated HPLC Variant II 155 (Bio-Rad Laboratories, Veenendaal, the Netherlands) and C-peptide concentration with Immulite XPI (Siemens, Eindhoven, the Netherlands). NAFLD was defined as ALT concentration above the upper limit of normal (ULN), corresponding to ALT>22.1 U/L for girls and ALT $>25.8 \mathrm{U} / \mathrm{L}$ for boys ${ }^{11}$. HOMA-IR was assessed using the formula: fasting glucose concentration $(\mathrm{mmol} / \mathrm{L})$ * fasting insulin concentration $(\mathrm{mU} / \mathrm{L}) / 22.5^{22}$.

\section{Statistical analyses}

Statistical analyses were performed using IBM SPSS Statistics for Windows version 24 (IBM Corp., Armonk, NY, USA). Subjects were divided into group 1 (ALT concentrations <ULN) 
and group 2 (ALT concentrations $>U L N$ ), and compared using factorial ANOVA's and MannWhitney-U tests. Changes over time were analysed with repeated measures ANOVAs.

To assess the effect of NAFLD activity in the whole group, the binary variable "NAFLD activity" was made in which subjects with concentrations below the ULN were numbered as " 0 " and those with ALT concentrations >ULN as "1". A multi-step regression model was used where sex, Tanner stage and BMI Z-score were added as co-variates to observe their effect on the overall model, as they have been associated with IR previously ${ }^{9,10}$. A two-sided p-value smaller $<0.05$ was considered statistically significant.

\section{RESULTS}

One hundred twenty-six adolescents were eligible for baseline measurements, and 83 (65.9\%) adolescents participated in follow-up measurements after 1y intervention. Dropouts and study completers did not differ in any of the variables measured at baseline (Dorenbos et al. unpublished results).

Differences in intervention outcomes between subjects with ALT concentrations above the ULN, indicating active NAFLD, and those with ALT concentrations below the ULN Participant characteristics are presented in Table 6.1. Fourty-one adolescents $(32.5 \%)$ presented with ALT concentrations $>U L N$, of which $43.9 \%$ were girls (compared to $65.9 \%$ in the group with ALT concentration $<U L N$ ). In group 2 (ALT concentrations $>U L N$ ) $43.9 \%$ were girls while in group 1 (ALT concentrations $>$ ULN) 65.9\% were girls, although this was not significant. Age and Tanner stage were not different between the two groups.

After 1y lifestyle intervention Tanner stage increased in both groups. Treatment*time analyses indicated that weight had increased significantly more in group 2 (ALT concentrations above ULN), compared to group 1 ( $p<0.05$, Table 1). BMI z-score decreased only in group $1(p<0.01)$ but not in group 2, and change in BMI $z$-score was statistically significantly different between the two groups $(p<0.05)$. ALT concentration increased in group 1 (ALT concentrations $>U L N$ ) while this remained stable in group 2 (ALT concentrations above ULN), and the change in ALT concentrations between the groups was statistically significant (treatment*time; $p<0.01$ ). Other changes in anthropometric characteristics and parameters of glucose metabolism were not significantly different between the two groups.

\section{Associations of ALT concentration, and ALT concentration above the ULN, with BMI} z-score and HOMA-IR at study onset and after one year of intervention

In a multivariate regression model in the whole group in which multiple co-variates (ALT concentration, BMI z-score, sex and Tanner stage) were entered, ALT concentration, sex and BMI z-score were identified as independent contributors to baseline HOMA-IR (Table 6.2A). 


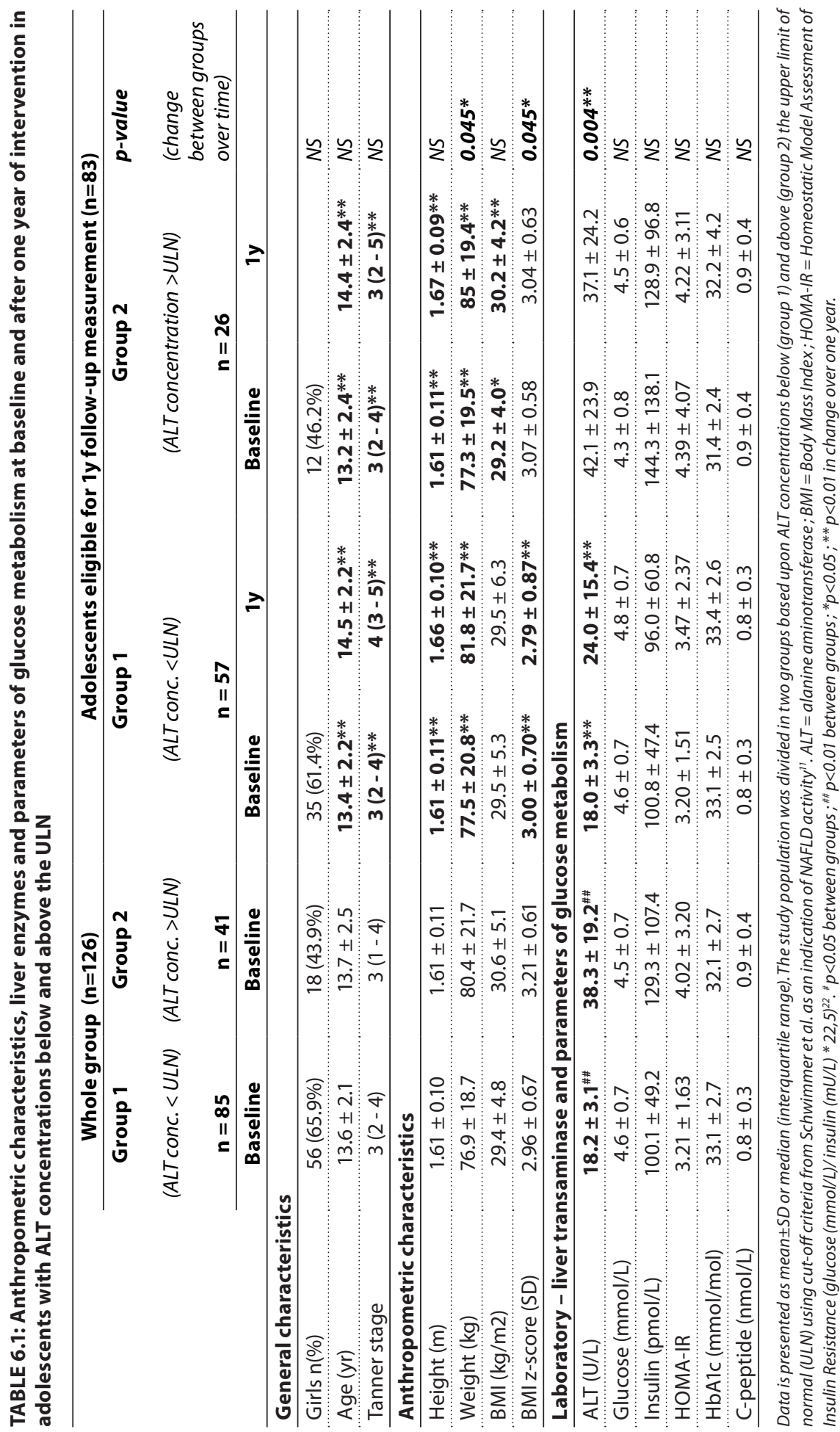


HOMA-IR and sex were associated with baseline BMI z-score. ALT concentration at baseline was not related to BMI z-score.

Associations of NAFLD activity with BMI z-score and HOMA-IR at baseline and after 1y intervention was assessed in multiple-step regression models (Table 6.2B). ALT concentration $>$ ULN, compared to ALT concentration $<U L N$, was positively associated with baseline BMI z-score although this disappeared after adding sex and Tanner stage as covariates. In addition, NAFLD activity was positively related to BMI z-score change after 1y. After adding sex and Tanner stage as co-variates the positive association of NAFLD with change in BMI $z$-score remained, although the overall model was no longer significant. NAFLD activity was not related to HOMA-IR at baseline, 1y or change herein. No collinearity was found between the factors included in these models.

\section{DISCUSSION}

This PREVIEW study aimed to assess the role of ALT concentration as a marker of NAFLD on outcomes of a combined lifestyle intervention, in particular BMI Z-score and HOMAIR, in adolescents with overweight/obesity. ALT concentrations >ULN, indicating NAFLD, were present in 1/3 of adolescents with overweight/obesity. Baseline ALT concentration was positively related to IR, although ALT concentration > ULN was not. After 1y PREVIEW intervention BMI z-score decreased significantly in the group of subjects with ALT concentrations $<U L N$, but not in those with abnormal ALT concentrations. NAFLD activity at baseline, compared to normal ALT concentration, was identified as a contributor to BMI z-score and change herein after 1 year lifestyle intervention.

A total of $32.5 \%$ of adolescents with overweight/obesity in this cohort presented with ALT concentrations >ULN. Prevalence of NAFLD was recently estimated to be $\sim 34 \%$ in children with obesity ${ }^{19}$, confirming this result of the present study. The golden standard for NAFLD diagnosis is liver biopsy, but due to the invasiveness of this procedure evaluation of ALT concentrations are more commonly used in paediatric practice. The ALT cutoff values used in this study have previously been shown to have a high sensitivity and specificity in both boys and girls and are widely used as a marker for NAFLD ${ }^{11}$. There were more boys in the group with elevated ALT concentrations compared to the group with normal ALT concentrations, although this was not statistically significant. Interestingly, ALT concentrations increased significantly in the group with normal baseline ALT, although mean ALT concentration at 1y did not surpass the ULN.

Baseline BMIz-score was not different between the group with normal ALT concentrations and those with ALT concentrations $>$ ULN. Adolescents with overweight/obesity and elevated ALT concentrations showed no change in BMI z-score after one year lifestyle intervention, while adolescents with normal ALT concentrations significantly decreased BMI z-score. The 
TABLE 6.2A: Prediction models for baseline BMI z-score and HOMA-IR

\begin{tabular}{|c|c|c|c|c|c|c|c|}
\hline & & B & SE & $95 \% \mathrm{Cl}$ & p-value & $\mathbf{R}^{2}$ & p-value model \\
\hline \multirow[t]{4}{*}{ BMI z-score model } & $\operatorname{ALT}(\mathrm{U} / \mathrm{L})$ & 0.002 & 0.004 & $(-0.006 ; 0.010)$ & 0.681 & 0.210 & $<0.001^{* *}$ \\
\hline & HOMA-IR & 0.099 & 0.025 & $(0.049 ; 0.149)$ & $<0.001^{* *}$ & & \\
\hline & Tanner stage & 0.050 & 0.040 & $(-0.030 ; 0.130)$ & 0.215 & & \\
\hline & Sex & 0.300 & 0.117 & $(0.069 ; 0.531)$ & $0.011^{*}$ & & \\
\hline \multirow[t]{4}{*}{ HOMA-IR model } & ALT (U/L) & 0.036 & 0.013 & $(0.010 ; 0.063)$ & $0.007^{* *}$ & 0.229 & $<0.001^{* *}$ \\
\hline & BMI z-score & 1.249 & 0.293 & $(0.568 ; 1.729)$ & $<0.001^{* *}$ & & \\
\hline & Tanner stage & 0.058 & 0.138 & $(-0.216 ; 0.332)$ & 0.675 & & \\
\hline & Sex & -0.942 & 0.400 & $(-1.734 ;-0.155)$ & $0.020^{*}$ & & \\
\hline
\end{tabular}

change in BMI z-score after 1y was statistically significant between both groups, and NAFLD activity was positively associated with baseline BMI z-score and change herein. At baseline this association was no longer significant after adding sex and Tanner stage as covariates, indicating that sex and puberty mediated this relationship. However, the positive association between NAFLD activity and BMI z-score change remained significant after adding sex and Tanner as co-variates into the regression analyses. These results indicate that NAFLD at onset was a risk factor for less $\mathrm{BMI}$ z-score decrease during intervention.

Although baseline ALT concentration was positively related to HOMA-IR, no significant associations were observed between ALT concentrations $>U L N$ and HOMA-IR or change herein. It is possible that the relation between NAFLD and IR is only indirect as our and others data showed a significant association between NAFLD and baseline IR only after adding sex and Tanner stage, although this was not observed after $1 y^{15,16,23}$. Furthermore, no change in HOMA-IR was observed in this study which may also have masked a possible effect of NAFLD activity on HOMA-IR. Since changes in HOMA-IR were shown to be primarily related to changes in BMI z-score (Dorenbos, submitted), similarly to previous observations, possible associations between NAFLD and IR may be indirect and depending on BMI z-score ${ }^{15}$.

As far as we know this is the first study assessing the effects of NAFLD on lifestyle intervention outcomes in adolescents with overweight/obesity. Limitations are the use of ALT concentration as a proxy for NAFLD activity due to the invasiveness of repeated liver biopsies and the relatively small sample size.

In conclusion, this study demonstrated that $1 / 3$ of adolescents with overweight/obesity presented with ALT concentrations above the ULN, indicative of NAFLD. Adolescents with elevated ALT concentrations had significantly less BMI z-score reduction after 1y lifestyle intervention. NAFLD was positively related to BMI z-score and change herein after $1 y$, suggesting that NAFLD might be a factor that affects lifestyle intervention outcome and success in adolescents with overweight/obesity. NAFLD was not directly associated with baseline IR or change herein. Although these results will have to be confirmed in larger cohorts, we recommend to screen for markers of NAFLD in childhood obesity interventions. 


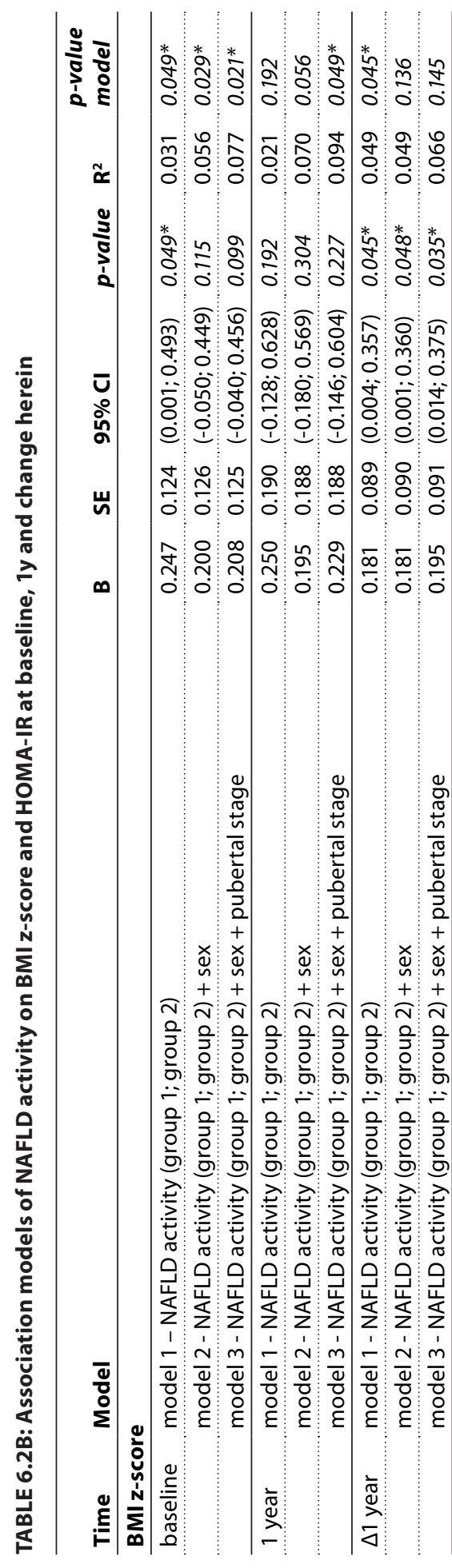

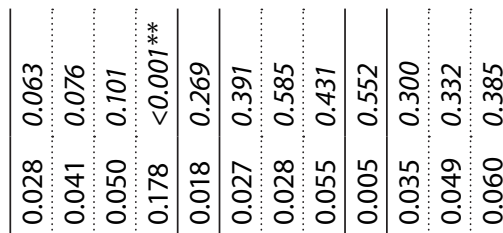

గి:

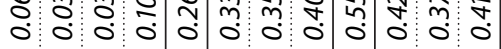
कि 난

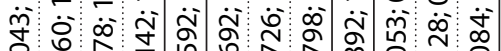

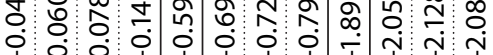

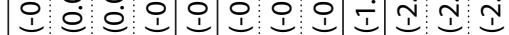
幽

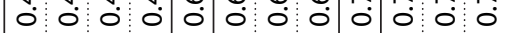

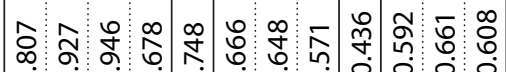

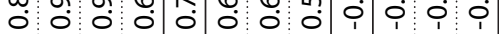

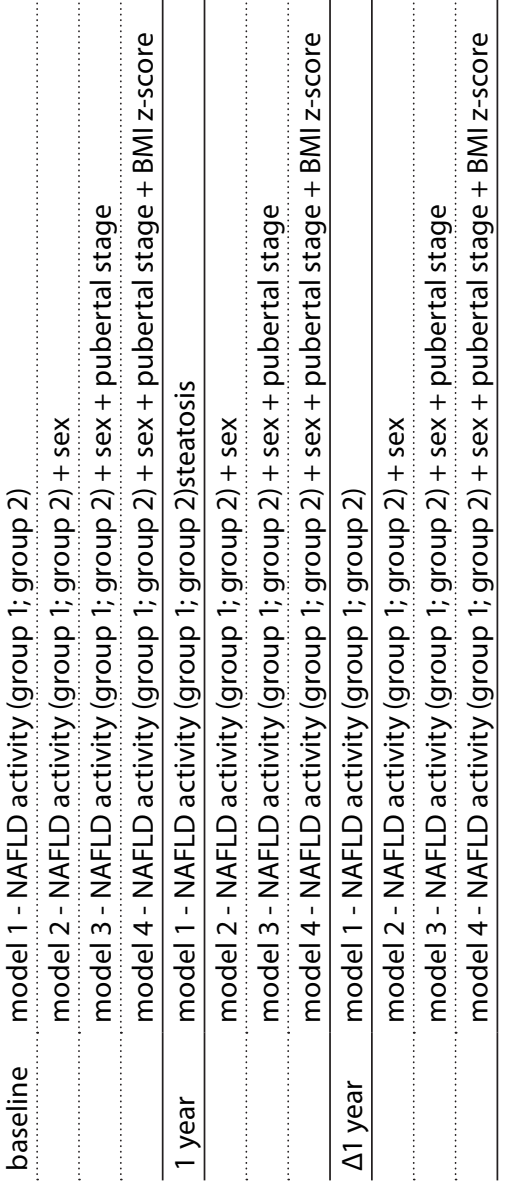

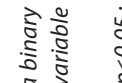
今े के .ેป ㄴํำ र 


\section{REFERENCES}

1. Lobstein T, Jackson-Leach R. Planning for the worst: estimates of obesity and comorbidities in school-age children in 2025. Pediatric obesity. 2016;11(5):321-325.

2. Lee JM, Okumura MJ, Davis MM, Herman WH, Gurney JG. Prevalence and determinants of insulin resistance among U.S. adolescents: a population-based study. Diabetes care. 2006;29(11):24272432.

3. van der Aa MP, Fazeli Farsani S, Knibbe CA, de Boer A, van der Vorst MM. Population-Based Studies on the Epidemiology of Insulin Resistance in Children. Journal of diabetes research. 2015;2015:362375.

4. van der Aa MP, Knibbe CA, Boer A, van der Vorst MM. Definition of insulin resistance affects prevalence rate in pediatric patients: a systematic review and call for consensus. Journal of pediatric endocrinology \& metabolism : JPEM. 2017;30(2):123-131.

5. Castro AV, Kolka CM, Kim SP, Bergman RN. Obesity, insulin resistance and comorbidities? Mechanisms of association. Arquivos brasileiros de endocrinologia e metabologia. 2014;58(6):600609.

6. Cree-Green M, Triolo TM, Nadeau KJ. Etiology of insulin resistance in youth with type 2 diabetes. Current diabetes reports. 2013;13(1):81-88.

7. Nightingale CM, Rudnicka AR, Owen CG, et al. Influence of adiposity on insulin resistance and glycemia markers among U.K. Children of South Asian, black African-Caribbean, and white European origin: child heart and health study in England. Diabetes Care. 2013;36(6):1712-1719.

8. Xu L, Li M, Yin J, et al. Change of Body Composition and Adipokines and Their Relationship with Insulin Resistance across Pubertal Development in Obese and Nonobese Chinese Children: The BCAMS Study. International journal of endocrinology. 2012;2012:389108.

9. AlKhater SA. Paediatric non-alcoholic fatty liver disease: an overview. Obesity reviews : an official journal of the International Association for the Study of Obesity. 2015;16(5):393-405.

10. Bush H, Golabi P, Younossi ZM. Pediatric Non-Alcoholic Fatty Liver Disease. Children (Basel, Switzerland). 2017;4(6):48.

11. Schwimmer JB, Dunn W, Norman GJ, et al. SAFETY study: Alanine aminotransferase cutoff values are set too high for reliable detection of pediatric chronic liver disease. Gastroenterology. 2010;138(4):1357-1364.e1352.

12. Jimenez-Rivera C, Hadjiyannakis S, Davila J, et al. Prevalence and risk factors for non-alcoholic fatty liver in children and youth with obesity. BMC Pediatrics. 2017;17(1):113.

13. Ozturk Y, Soylu OB. Fatty liver in childhood. World journal of hepatology. 2014;6(1):33-40.

14. Cruz ML, Shaibi GQ, Weigensberg MJ, Spruijt-Metz D, Ball GD, Goran MI. Pediatric obesity and insulin resistance: chronic disease risk and implications for treatment and prevention beyond body weight modification. Annual review of nutrition. 2005;25:435-468.

15. Gruben N, Shiri-Sverdlov R, Koonen DP, Hofker MH. Nonalcoholic fatty liver disease: A main driver of insulin resistance or a dangerous liaison? Biochimica et biophysica acta. 2014;1842(11):23292343.

16. Valenti L, Bugianesi E, Pajvani U, Targher G. Nonalcoholic fatty liver disease: cause or consequence of type 2 diabetes? Liver international : official journal of the International Association for the Study of the Liver. 2016;36(11):1563-1579.

17. Baxter KA, Ware RS, Batch JA, Truby H. Predicting success: factors associated with weight change in obese youth undertaking a weight management program. Obesity research \& clinical practice. 2013;7(2):e147-e154.

18. Chiavaroli V, Giannini C, D'Adamo E, et al. Weight loss in obese prepubertal children: the influence of insulin resistance. Endocrine research. 2013;38(1):48-57.

19. Yu EL, Golshan S, Harlow KE, et al. Prevalence of Nonalcoholic Fatty Liver Disease in Children with Obesity. The Journal of pediatrics. 2018. 
20. Dorenbos E, Drummen M, Rijks J, et al. PREVIEW: Prevention of diabetes through lifestyle intervention in a multicentre study in Europe in children (10-17y). Design, methods, and baseline results. Diabetes, obesity \& metabolism. 2018;20(5):1096-1101.

21. de Onis M, Onyango AW, Borghi E, Siyam A, Nishida C, Siekmann J. Development of a WHO growth reference for school-aged children and adolescents. Bull World Health Organ. 2007;85(9):660-667.

22. Matthews DR, Hosker JP, Rudenski AS, Naylor BA, Treacher DF, Turner RC. Homeostasis model assessment: insulin resistance and beta-cell function from fasting plasma glucose and insulin concentrations in man. Diabetologia. 1985;28(7):412-419.

23. Lonardo A, Ballestri S, Marchesini G, Angulo P, Loria P. Nonalcoholic fatty liver disease: a precursor of the metabolic syndrome. Digestive and liver disease : official journal of the Italian Society of Gastroenterology and the Italian Association for the Study of the Liver. 2015;47(3):181190. 


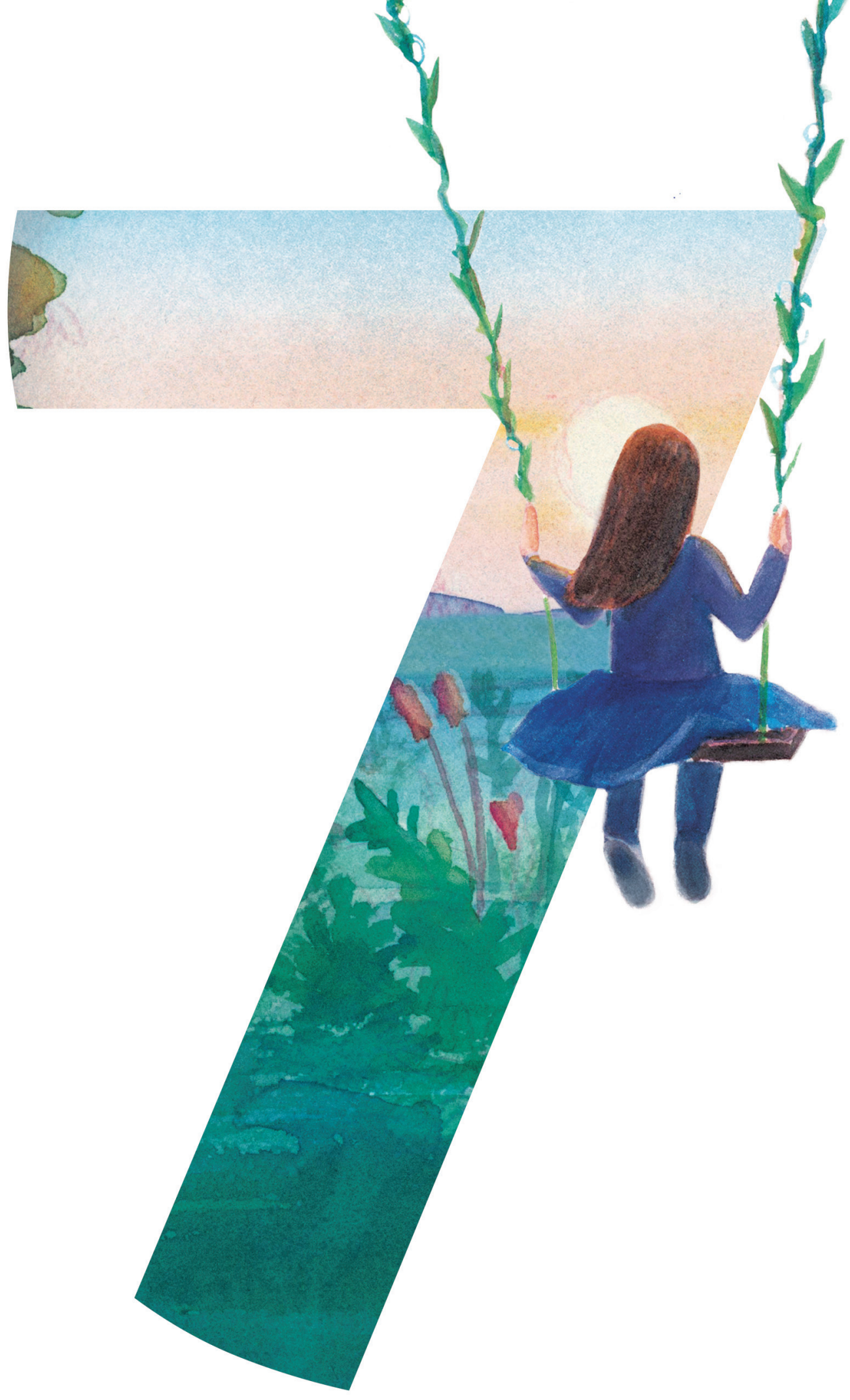




\section{Chapter 7}

General discussion 
This thesis aimed to evaluate the effects of lifestyle intervention for the treatment of high pubertal insulin resistance in adolescents with overweight and obesity, and to gain new insights in determinants of pubertal insulin resistance, specifically insufficient duration and quality of sleep, and non-alcoholic fatty liver disease (NAFLD).

The prevention of diabetes through lifestyle intervention and population studies in Europe and around the world (PREVIEW) study was a large, international randomized controlled trial assessing the most effective combination of dietary and physical activity strategies to prevent type 2 diabetes mellitus (T2DM) in adults and adolescents ${ }^{1}$. The aim of the PREVIEW study in adolescents was to assess the effects of a high-protein low-Gl diet (HP, 25/45/30 En\% protein/carbohydrate/fat, GI >56) or moderate-protein moderate-GI diet (MP, $15 / 55 / 30$ En\% protein/carbohydrates/fat, GI <50) on IR in adolescents with overweight and obesity at increased risk for developing T2DM. It was hypothesized that the HP diet would be superior in reducing IR compared to the MP diet. The second aim of the PREVIEW study was to assess the role of possible risk factors for T2DM development, specifically sleep and non-alcoholic fatty liver disease, on intervention-related outcomes.

\section{COMPLIANCE TO AND FEASIBILITY OF THE LONG-TERM COMBINED LIFESTYLE INTERVENTION}

\section{Attrition}

Motivation to complete the PREVIEW study was less than expected. Based upon general recommendations this study used an expected drop-out of $25 \%$ in power analyses to assess sample size ${ }^{2}$. In this study however retention rates were $66 \%$ after one year and $39 \%$ after two years. In the sleep substudy $40 \%$ of participants were willing to participate in a second overnight hospital stay for polysomnography measurement after one year. The main reason for study discontinuation was loss to follow up. Although no qualitative research was performed and the real rationale for study discontinuation are therefore not known, problems with recruitment and retention are common in childhood obesity studies and some reasons can be learned from earlier trials ${ }^{2}$. Frequently mentioned arguments are misalignment of the research purpose and methods with the goals and lifestyle of adolescents that would have to adhere to them, as was also described in a qualitative survey of the MIKADO study performed under drop-outs (Willeboordse, unpublished data). In the Centre for Overweight Adolescent and Children's Healthcare (COACH) cohort, from which a large part of the participants for the PREVIEW study were recruited, attrition was $10 \%$ after one year but increased to $30 \%$ and $50 \%$ after two and three years ${ }^{3}$ (and unpublished data). One of the most used reasons for discontinuation was that participants felt that they had learned sufficiently about healthy lifestyle. In this cohort, the main reason for drop-out was also the program not meeting the patient's expectations, or the feeling that patients had 
gained enough knowledge on healthy lifestyle. A meta-analysis reported that drop-out rates in other lifestyle intervention studies for children with overweight/obesity varied between 2 and $51 \%$ at 12 months $^{4}$. Considering the intensive, long-term nature of the PREVIEW study and that most of the PREVIEW participants had already participated in one or more years of $\mathrm{COACH}$ intervention, the attrition rates seem in line with those of previous studies.

However, as high attrition is such a common problem in adolescent (obesity) research and was also an obstacle in the PREVIEW study, some recommendations can be made to help future adolescent obesity research ${ }^{2}$. A recent study assessed the effect of consumer involvement in an adolescent obesity study, and found that inclusion of fun games, practical activities, resistance training and forming new friendships with other participants resulted in an impressive $82 \%$ and $62 \%$ of participants completing measurements at one and two years, respectively ${ }^{5-7}$. Based upon our and previous studies' experiences, it might be helpful to involve adolescents in the design of the study, use more age-appropriate tools (e.g. e-health), and plan regular contacts and fun social activities to enhance study completion and compliance ${ }^{2,6}$. Furthermore, if interim analyses show that study compliance or completion is severely affected, as was demonstrated by the PREVIEW study where attrition after two years was very low and therefore not suitable for completer analyses, small adjustments to the study protocol might be considered to enhance overall compliance and usability of the study data.

\section{Compliance to dietary instructions}

Dietary instructions were provided during individual meetings with adolescents and their parents, which took place during regular clinical investigation days. All adolescents were provided with sample menus in line with their randomization group and individual dietary needs. After one and two years of dietary instructions, no significant changes in protein intake were observed between different timepoints or between the HP and MP intervention groups, despite the groups receiving different dietary instructions. However, total reported energy intake, as well as the absolute intake of fat and carbohydrates decreased significantly after one year of personalized dietary counselling. Participants also reported an increase in dietary restraint on the Three-Factor Eating Questionnaire after one and two years of study participation, indicating a more conscious eating pattern ${ }^{8}$.

Thus far, seven studies have been conducted assessing the effects of a high-protein diet on BMI z-score in adolescents with overweight and obesity ${ }^{9-15}$, of which five reported a significant increase in dietary protein intake at 12 weeks ${ }^{11,12}, 26$ weeks ${ }^{13,14}$ or 12 months ${ }^{15}$, and two did not observe a difference in protein intake. All of the studies set in free-living conditions reported difficulties in dietary compliance ${ }^{9-13,15,16}$. If an increase in protein intake was observed, protein intake was on average $\sim 21 \mathrm{En} \%$, which was less than the targets set between 22.5 and $25 \mathrm{En} \%$. 
There are a couple of reasons that might explain why an increase in protein intake could not be achieved in the PREVIEW study. Firstly, the method of assessing dietary compliance was different. The PREVIEW study assessed food intake using self-reported food diaries because they were easy to use, were available on an app, and allowed for quick and easy evaluation and feedback during measurement contacts. Although this is the most frequently used tool for dietary assessment in research settings, food diaries are known to often underreport actual food intake ${ }^{17,18}$. This might have been the case in the PREVIEW study, too, as adolescents reported a markedly lower total energy intake compared to their estimated energy requirements from the $\mathrm{WHO}$ formula ${ }^{19}$. Moreover, the increase in weight and $\mathrm{BMI}$ sec implicate that in reality, an increase in energy intake must have taken place. The data of this study therefore does not allow conclusions on whether the absence of differences in protein intake truly is a result of insufficient dietary compliance. Other methods that have been previously used in adolescent studies are meal observation in an in-centre design or a 3-pass $24 \mathrm{~h}$ dietary recall, although the latter is self-reported and therefore also presents with problems in reliability ${ }^{9-11,13,15}$. While these methods may improve validity of the reported food intake, it might not be representative of normal eating behaviour. An alternative is the use of urine-nitrogen sampling as was performed by the PREVIEW study in adults, but this was not deemed to be safe for adolescents'.

Considering design, the PREVIEW study was set in free-living conditions as opposed to the in-centre design ${ }^{9-11}$ or supervised grocery shopping designs as used in the studies ${ }^{13,14}$. While this is more representative of normal living circumstances, a free-living design also allows participants more freedom to (not) adhere to the dietary guidelines. This study also differed from the other studies in that instructions were highly personalized and tailored on the needs of the adolescent, instead of focussed on the whole family, and had fewer contact moments than some other studies ${ }^{11-15}$. Although this made the PREVIEW study less intensive than some of the previous studies, it might have negatively affected dietary compliance. It should also be noted that Garnett et al. provided additional metformin medication, which is known to enhance BMI z-score reduction and might therefore have influenced motivation and study compliance ${ }^{15}$.

One last important difference is that the study duration of the PREVIEW study was longer than in the previously conducted studies, where the intervention ranged from 12 weeks to 12 months. Longer study duration might have compromised dietary compliance, as was also observed in the study by Mirza et al. where protein intake was significantly increased at 12 weeks of active intervention, but not after 12 and 24 months when adolescents received ambulatory instructions ${ }^{11}$. One study in adults demonstrated that protein intake stimulated satiety centres in the brain but reduced reward mechanisms, which may explain difficulties in maintaining high protein diets for an extended period of time ${ }^{20}$. 
In conclusion, the dietary intervention of the study as it was designed was not feasible. The absence of a significant difference in protein intake in adolescents in this study may be attributable to under- or misreporting of actual food intake, the free-living design, the relatively long duration of the trial and the effects of protein on the brain reward system. The results of the PREVIEW study and previous protein studies in adolescents do however demonstrate that maintaining and achieving high protein intake for an extended period of time is not feasible in adolescent populations in free-living conditions. It is possible that this is only achievable long-term using vouchers or subsidies for products high in protein, meal replacement, or with protein supplements.

\section{Compliance to physical activity instructions}

Physical activity instructions consisted of encouragement to increase physical activity of any sort. In the PREVIEW study in adolescents, physical activity was not a part of the RCT and therefore not protocolized. However, as physical activity is an important part of health recommendations for childhood obesity, PA recommendations were provided and exercise in general was encouraged on a regular basis. Instructions were highly personal and mainly focused on participants finding and continuing on a form of exercises they enjoyed to encourage long-term compliance. So, if a participant did already participate in sports, they were encouraged to maintain and expand on this. If they did not already participate in any form of exercise, they were invited to join weekly physical activity orientation classes especially designed for adolescents with overweight and obesity. In addition to these instructions, all adolescents received booklets specifically designed for the PREVIEW study, with tutorials and exercised they could perform at home.

During the PREVIEW study, measurements after one and two years showed a significant increase in accelerometry counts, moderate, vigorous and moderate-to-vigorous activity while sedentary time was decreased. This is particularly encouraging since an earlier observational study found that PA generally decreased for each year that a child aged, mainly caused by a relative increase in time spent sedentary ${ }^{21}$. It should also be considered that although counts provide a quantification of the performed activity, it does not per se quantify energy expenditure. Indeed, earlier studies have indicated that in individuals with higher weight and BMI, PA counts may be lower but energy expenditure per activity is higher compared to lean individuals ${ }^{22,23}$. In our study it is likely that the increase in PA is attributable to the personalized coaching and the focus on making sports a habitual, fun activity. 


\section{OBSERVATIONS DURING THE PREVIEW INTERVENTION}

\section{HOMA-IR stabilized after one year of study participation, despite progression in pubertal status}

Even though the incidence of T2DM in youth is increasing, the study of preventative strategies for T2DM would require very large cohorts and long follow-up time, which propels most studies in adolescents to use IR as a precursor for diabetes. As discussed in the introduction of this thesis pubertal IR is a complicated and challenging research area, as it is both a physiological process but also poses an increased risk for developing T2DM and other normally late-onset morbidities. It is therefore the question to what extend IR should be meddled with. Currently there is enough evidence to ascertain that IR during puberty is necessary to facilitate normal tissue proliferation and growth, but that increased $\mathrm{IR}$ - especially in the presence of other risk factors such as obesity - severely increases the risk of an adolescent to develop T2DM and related morbidity ${ }^{24-26}$. Therefore, especially the monitoring and prevention of further IR increase is important.

One of the most important determinants of IR is obesity status. Cross-sectional studies have shown a comparable but enhanced pattern in adolescents with overweight and obesity, where HOMA-IR is higher than in lean peers at the onset of puberty, reaches higher values at mid-puberty, and does not always decrease as adolescents enter adulthood $\mathrm{d}^{25,27-33}$. A similar effect was observed in two studies described in this thesis. The study presented in CHAPTER 2 of 137 adolescents (aged 10-18) with overweight and obesity demonstrated that postpubertal boys (Tanner stages 4-5) had significantly higher HOMA-IR than prepubertal boys, although this effect was not found in girls ${ }^{34}$. This was confirmed in the baseline descriptives of the 126 adolescents (age 10-17) with overweight/obesity and IR that participated in the PREVIEW study (CHAPTER 3). Here, too, pubertal (Tanner stages 3-5) adolescents with morbid obesity demonstrated significantly higher HOMA-IR values compared to prepubertal adolescents with morbid obesity, and to prepubertal and pubertal adolescents with overweight and obesity ${ }^{35}$. Earlier studies showed that higher HOMA-IR in individuals with overweight/obesity might be a result of the increased ectopic fat storage related to obesity ${ }^{36,37}$. It has been postulated that persistent IR at the end of puberty, especially in those with morbid obesity, puts these postpubertal adolescents at especially high risk for $\beta$-cell exhaustion and T2DM development ${ }^{25,27,34}$.

Mean HOMA-IR did not change significantly after one year of intervention but increased after two years. As described previously, IR of puberty typically nadirs at mid-puberty (CHAPTER 4). The stabilization of HOMA-IR after one year of intervention despite progression in pubertal state indicates that IR did not exacerbate further in the first year of intervention.

Change in HOMA-IR after one year of study participation was positively related to change in BMI z-score, and HOMA-IR stabilized or decreased in individuals that showed a significant reduction in BMI z-score. In addition, HOMA-IR and BMI z-score were consistently 
positively related in cross-sectional analyses at baseline and after one year of intervention. No direct associations were found between possible changes in food intake or PA with HOMA-IR change. It is possible that the stabilization of HOMA-IR despite the progression in pubertal stage in our cohort is due to the overall decrease in BMI z-score, or that the intraindividual change in HOMA-IR after one year in puberty is smaller than what is reflected in the previously published cross-sectional studies ${ }^{25,27}$. The cross-sectional increase in HOMAIR in those studies might also be related to differences in mean BMI z-score per Tanner stage group. Nonetheless, the results from this study indicate that BMI z-score is one of the most important independent determinants of HOMA-IR in adolescents with overweight and obesity, as was shown by a constant positive relationship between IR and BMI Z-score, IR being the highest in adolescents with the most severe obesity grade, and stabilization of IR after one year as BMI Z-score decreased. This was a confirmation and extension of earlier studies observing a consistent positive association between BMI z-score and IR in adolescents $25,36,37$.

After two years of PREVIEW intervention mean HOMA-IR increased as pubertal stage progressed further. BMI z-score had decreased significantly from baseline measurements (but not compared to data at 1 year of intervention). It is possible that this increase in IR is attributable to the peak in IR normally shown in mid-puberty, while relative BMI z-score change between one and two years of PREVIEW intervention was too small to counteract for this. However, these results do indicate a persistence of high IR at the end of puberty in adolescents with overweight and obesity in our data, as has also been suggested by a longitudinal study on pubertal IR by Goran et $a^{\beta 1}$. No further longitudinal studies have been published on pubertal IR development in adolescents with overweight/obesity, but crosssectional observations from the COACH study and PREVIEW baseline study (CHAPTERS 2 \& 3), as well as that from other large cross-sectional studies, also indicate that adolescents with especially higher grades of obesity show a persistent instead of transient high IR during puberty ${ }^{27,29}$. Although these findings should be confirmed in larger studies with long followup, they do emphasize the necessity to prevent further IR exacerbation during puberty, especially in adolescents with overweight and obesity.

\section{BMI z-score was significantly reduced after one and two years of dietary and}

\section{PA counselling}

Post-hoc analyses showed that after one year of PREVIEW participation mean BMI z-score decreased with -0.17 SD in adolescents with overweight/obesity and IR, and after two years this further decreased with -0.19 SD (CHAPTER 4). Recent meta-analyses of lifestyle intervention studies in children with overweight and obesity (but not necessarily IR) showed that a reduction of $\geq-0.15$ SD was related to significant clinical improvements in insulin sensitivity, lipid concentration (specifically total cholesterol and LDL-cholesterol concentrations), and normalization of blood pressure ${ }^{38,39}$. Cochrane meta-analyses recently 
observed a mean BMI z-score reduction of -0.14 SD $(95 \% \mathrm{Cl}-0.17 ;-0.12)$ after 6 months and -0.14 SD $(95 \% \mathrm{Cl}-0.18 ;-0.10)$ at 12 months, which is less than the BMI z-score decrease that was observed in our present study ${ }^{4,40}$. Moreover, in previous lifestyle intervention studies those individuals with IR did not decrease BMI z-score after one to fourteen years or even increased their BMI z-score during intervention ${ }^{41-45}$. It is therefore promising that in this study, where participants were selected based upon their IR status, a BMI z-score decrease was observed that was both statistically significant and clinically relevant and which did not rebound after a prolonged time period. It should be mentioned that weight and BMI did increase during the study, even as BMI z-score decreased. This indicates that although an increase in energy intake must have taken place to result in these changes, this increase was relatively mild in relation to $\mathrm{PA}$ and growth to yield an overall $\mathrm{BMI}$ z-score reduction.

Increased dietary restraint scores on the TFEQ were related to reduction of BMI z-score after one year in this population, indicating that individuals that acquired more conscious and controlled eating behaviours showed a reduction in BMI z-score. Increase in TFEQ hunger scores was positively related with BMI z-score change, indicating that those with increased hunger susceptibility (possibly as an effect of more controlled eating behaviours) did not decrease BMI z-score. This might be partly explained by genetic predispositions ${ }^{8}$. No associations were found between changes in PA and BMI z-score.

\section{No changes were observed in parameters of lipids or inflammation}

After one and two years of intervention, a small but significant increase in fasting glucose concentration was observed which at two years also resulted in a higher HOMA-IR. After one year a small increase in ALT concentrations was detected in the imputed data, although this was not observed in the measured data of the completers at one year. No changes were observed in other parameters of glucose metabolism, lipids or inflammation parameters. Although some studies did observe a decrease in systolic and diastolic blood pressure or lipids at the end of the active intervention period, this was not confirmed in our and others studies ${ }^{10,11,15,16}$. Participants in our study presented with mean values within normal ranges for blood pressure, lipids, and parameters for liver function and inflammation at study onset, it is therefore possible that no change was observed since these values were already within the healthy range.

\section{RISK FACTORS FOR BMI Z-SCORE, IR AND INTERVENTION-RELATED HEALTH OUTCOMES}

\section{Habitual short sleep duration was positively related to BMI z-score}

As described previously, the second aim of the PREVIEW study was to assess the role of other risk factors that might be associated with BMI z-score and HOMA-IR change during the 
PREVIEW intervention. Short sleep duration and sleep debt have been extensively related to increased risk of developing obesity in adults and adolescents, and to lesser extent, pubertal $\mathrm{IR}^{46-48}$. Sleep has therefore been proposed to be, in addition to food intake and PA, a third modifiable contributor to the development of obesity and IR $\mathrm{R}^{46,48-54}$.

For the study presented in CHAPTER 2, 137 adolescents with overweight or obesity were subjected to a cross-sectional polysomnography measurement. In prepubertal girls, but not girls in other pubertal stages or in boys, both total sleeping time (TST) and sleep efficiency (SE, calculated as TST/time in bed) were negatively related to HOMA-IR. This indicated that inadequate sleep duration may indeed negatively affect IR, possibly because longer sleep is a protective factor for glucose metabolism and pancreatic $\beta$-cell compensation ${ }^{26}$.

In the follow-up study presented in CHAPTER 5, 67 adolescents with overweight/ obesity and IR of the PREVIEW study were subjected to a polysomnography. Habitual total sleeping time was inversely related to baseline BMI z-score and absolute fat mass and fat free mass, even after correcting for pubertal stage. This indicated that short sleep was not only a risk factor for developing overweight, but is also related to higher BMI z-score in adolescents that were overweight/obese at the start of the intervention. No associations were observed between parameters of sleep architecture and anthropometric or cardiometabolic outcomes, suggesting that overall absolute habitual sleep was more important in obesity than sleep quality. In 29 adolescents sleep assessments were repeated after one year of study participation. No decline in sleep duration was observed, which might be due to either the low number of adolescents participating in the follow-up study or the relatively short follow-up time. Possibly also as a consequence of stabilization of sleep duration and small sample size, no associations were observed between changes in parameters of sleep duration and architecture, and changes in anthropometric and cardiometabolic health parameters.

The observation that habitual sleep was associated with BMI z-score at study onset is a confirmation of earlier studies that found a similar relationship in cohorts consisting of lean children and children with overweight/obesity. This might be explained by a few reasons, as presented by Quist et al $^{48}$. First, shorter sleeping duration results in more opportunity to eat, which especially later in the day is often marked by intake of high-caloric foods and drinks. Second, inadequate sleep has been related to changes in hormones, particularly a decrease in leptin and increase in ghrelin, as well as influencing reward-related brain functions leading to a preference for energy-dense foods. This can also lead to higher energy intake. Lastly, inadequate sleep results in more daytime fatigue, which can lead to making less healthy food choices and a decrease in energy expenditure, while sedentary time is increased. Unfortunately, the sample size of this exploratory study was too small to study associations between sleep duration and physical activity or dietary intake. We can therefore only conclude that sleep is a risk factor for higher BMI z-score in adolescents even in those that were overweight/obese at study onset, and sleep hygiene might be a target for 
prevention and treatment of obesity in youths, although this should be confirmed in future longitudinal studies.

The latter part of CHAPTER 5 presents a comparison of objective and subjective measurements of sleep assessment, which are often used in paediatric research. The wide variety of sleep assessment methods limits the comparison of sleep studies performed in childhood cohorts. The golden standard for sleep assessment is polysomnography, but this is an invasive procedure (polysomnography requires overnight in-hospital measurements) as well as a costly and time-intensive one. Other commonly used methods are habitual sleep measurement using actigraph, and the use of sleep questionnaires such as the Pittsburgh Sleep Quality Index (PSQI), where higher scores indicate poorer sleep quality ${ }^{55}$. In this PREVIEW study both objective and self-reported sleep assessments were compared to each other. We observed that self-reported poor sleep quality on the PSQI questionnaire was inversely related to percentage of slow-wave and REM sleep, indicating deep sleep on the polysomnography. Furthermore, poor sleep duration scores were inversely related to habitual sleeping time, and wake-after-sleep-onset was related to self-reported daytime dysfunction scores. Although PSQI scores were indicative of polysomnography outcomes, polysomnography-measured and habitual sleep duration were not interrelated and only habitual sleep duration was related to anthropometric characteristics. It is possible that this is because habitual sleep measurement by actigraph was measured during 4 consecutive nights at home, while polysomnography was measured during an overnight stay at the hospital. These results do however stress that objective and self-assessed sleep measurements measure different dimensions of sleep and may complement each other when combined.

Increased aminotransferase concentration, indicative of NAFLD, was associated with IR and less BMI z-score decrease

IR and non-alcoholic fatty liver disease often co-develop in adolescents and are suggested to influence each other ${ }^{56-63}$. Since IR appeared to be a risk factor for BMI z-score decrease during lifestyle intervention in children with obesity, we hypothesized that NAFLD might also affect intervention-related outcomes. A substudy of PREVIEW, presented in CHAPTER $\mathbf{6}$, was therefore conducted to assess the possible role of NAFLD on BMI z-score, IR, and intervention-related outcomes of the lifestyle intervention.

NAFLD was defined using alanine aminotransferase (ALT) concentrations above the upper limit of normal (ULN) values of $>22.1 \mathrm{U} / \mathrm{L}$ for girls and $>25.8 \mathrm{U} / \mathrm{L}$ for boys ${ }^{64} .32 .5 \%$ of adolescents with overweight and obesity in this cohort presented with ALT concentrations above the ULN, which is higher than the estimated $3-13 \%$ in the general population but similar to an earlier study where $1 / 3^{\text {rd }}$ of adolescents with obesity showed signs of NAFLD ${ }^{65}$.

Baseline ALT concentrations and BMI z-score were independently related to HOMAIR after correcting for Tanner stage and sex. ALT concentration >ULN, indicating NAFLD, 
was however not independently related to HOMA-IR or change herein, and only positively related to baseline HOMA-IR after adding sex and Tanner stage as covariates. This indicated that NAFLD has no direct effect on IR in adolescents, but rather that its relation with HOMAIR was mediated through BMI z-score change, which we and others have shown to be an important mediator of pubertal IR $\mathrm{R}^{61-63}$.

An important result of this study was that adolescents with baseline ALT concentrations above the ULN showed no BMI z-score decrease, while BMI z-score decreased in adolescents with normal ALT concentrations, and this difference was statistically significant. This indicated that elevated ALT concentration, indicating active NAFLD, at the start of lifestyle intervention negatively affected outcomes in adolescents with overweight and obesity. These results should be confirmed in larger studies, which should also further research the underlying mechanisms. However, as the results of this study clearly identify NAFLD as a risk factor for less BMI z-score decrease, we advise screening for NAFLD prior to the start of lifestyle intervention strategies in adolescents with overweight or obesity.

\section{CONCLUSIONS}

In conclusion, the results of the PREVIEW study add new insights into the effects of a highprotein low-Gl and moderate-protein moderate-GI diet on insulin resistance in adolescents with overweight and obesity who are at high risk to develop T2DM. Pubertal adolescents with morbid obesity were found to be at highest risk of T2DM development by exhibiting persistence of IR at the end of puberty, which was significantly higher than observed in adolescents that had overweight/obesity or were prepubertal.

No significant differences in reported protein intake and Gl were observed between the two intervention groups, in spite of the different dietary instructions that that were provided to each group. In addition, no differences were observed between the intervention groups regarding IR, parameters of glucose or lipid metabolism, inflammation, liver transaminases, nor lifestyle factors at any timepoint. We therefore conclude that the dietary strategy as it was designed was not feasible.

Changes in reported protein intake were not significantly different between timepoints and no effects of the intervention on IR was found. Nonetheless, HOMA-IR stabilized after one year of intervention and increased slightly after two years. The stabilization in HOMAIR after one year was positively related to change in BMI z-score. Post-hoc analyses also showed a significant and clinically relevant overall reduction of BMI z-score of -0.17 SD after one year, and - 0.19 SD after two years of PREVIEW dietary and PA instructions. The reduction in BMI z-score was related to an increase in dietary restraint.

Shorter habitual sleep duration was related to higher BMI z-score at study onset, but changes in sleep duration or parameters were not related to changes in BMI z-score or HOMA-IR. 
Alanine aminotransferase concentrations above the upper limit of normal, indicative of NAFLD, were a risk factor for less BMI z-score decrease after one year of intervention but not directly related to HOMA-IR.

Based upon the observations of the PREVIEW study in adolescents, we advise to screen adolescents for NAFLD prior to commencement of lifestyle intervention. Lifestyle intervention strategies aiming to decrease IR and BMI z-score in adolescents with overweight/ obesity should aim to increase awareness of dietary intake by increasing cognitive restraint, as this was found to be a target for BMI z-score reduction and IR stabilization in adolescents with overweight/obesity at high risk for developing T2DM. 


\section{REFERENCES}

1. Fogelholm M, Larsen T, Westerterp-Plantenga $M$, et al. PREVIEW: Prevention of Diabetes through Lifestyle Intervention and Population Studies in Europe and around the World. Design, Methods, and Baseline Participant Description of an Adult Cohort Enrolled into a Three-Year Randomised Clinical Trial. Nutrients. 2017;9(6):632.

2. Steinbeck K, Baur L, Cowell C, Pietrobelli A. Clinical research in adolescents: challenges and opportunities using obesity as a model. International journal of obesity (2005). 2009;33(1):2-7.

3. Rijks JM, Plat J, Mensink RP, Dorenbos E, Buurman WA, Vreugdenhil AC. Children With Morbid Obesity Benefit Equally as Children With Overweight and Obesity From an Ongoing Care Program. The Journal of clinical endocrinology and metabolism. 2015;100(9):3572-3580.

4. Oude Luttikhuis $\mathrm{H}$, Baur $\mathrm{L}$, Jansen $\mathrm{H}$, et al. Interventions for treating obesity in children. The Cochrane database of systematic reviews. 2009(1):Cd001872.

5. Shrewsbury VA, O'Connor J, Steinbeck KS, et al. A randomised controlled trial of a communitybased healthy lifestyle program for overweight and obese adolescents: the Loozit ${ }^{\oplus}$ study protocol. BMC Public Health. 2009;9(1):119.

6. Nguyen B, Shrewsbury VA, O'Connor J, et al. Two-year outcomes of an adjunctive telephone coaching and electronic contact intervention for adolescent weight-loss maintenance: the Loozit randomized controlled trial. International journal of obesity (2005). 2013;37(3):468-472.

7. Nguyen B, Shrewsbury VA, O'Connor J, et al. A process evaluation of an adolescent weight management intervention: findings and recommendations. Health promotion international. 2015;30(2):201-212.

8. Vogels N, Posthumus DL, Mariman EC, et al. Determinants of overweight in a cohort of Dutch children. The American journal of clinical nutrition. 2006;84(4):717-724.

9. Rolland-Cachera MF, Thibault $\mathrm{H}$, Souberbielle JC, et al. Massive obesity in adolescents: dietary interventions and behaviours associated with weight regain at 2 y follow-up. International journal of obesity and related metabolic disorders : journal of the International Association for the Study of Obesity. 2004;28(4):514-519.

10. Gately PJ, King NA, Greatwood HC, et al. Does a high-protein diet improve weight loss in overweight and obese children? Obesity (Silver Spring, Md). 2007;15(6):1527-1534.

11. Mirza NM, Palmer MG, Sinclair KB, et al. Effects of a low glycemic load or a low-fat dietary intervention on body weight in obese Hispanic American children and adolescents: a randomized controlled trial. The American journal of clinical nutrition. 2013;97(2):276-285.

12. Baxter KA, Ware RS, Batch JA, Truby H. Predicting success: factors associated with weight change in obese youth undertaking a weight management program. Obesity research \& clinical practice. 2013;7(2):e147-e154.

13. Papadaki A, Linardakis M, Larsen TM, et al. The effect of protein and glycemic index on children's body composition: the DiOGenes randomized study. Pediatrics. 2010;126(5):e1143-1152.

14. Damsgaard CT, Papadaki A, Jensen SM, et al. Higher protein diets consumed ad libitum improve cardiovascular risk markers in children of overweight parents from eight European countries. The Journal of nutrition. 2013;143(6):810-817.

15. Garnett SP, Gow M, Ho M, et al. Improved insulin sensitivity and body composition, irrespective of macronutrient intake, after a 12 month intervention in adolescents with pre-diabetes; RESIST a randomised control trial. BMC Pediatr. 2014;14:289.

16. Duckworth LC, Gately PJ, Radley D, Cooke CB, King RF, Hill AJ. RCT of a high-protein diet on hunger motivation and weight-loss in obese children: an extension and replication. Obesity (Silver Spring, Md). 2009;17(9):1808-1810.

17. Heitmann BL, Lissner L, Osler M. Do we eat less fat, or just report so? International journal of obesity and related metabolic disorders : journal of the International Association for the Study of Obesity. 2000;24(4):435-442. 
18. Kant AK, Graubard BI. Secular trends in patterns of self-reported food consumption of adult Americans: NHANES 1971-1975 to NHANES 1999-2002. The American journal of clinical nutrition. 2006;84(5):1215-1223.

19. Organization WH. Human energy requirements. Report of a Joint FAO/WHO/UNU Expert Consultation. 2004.

20. Born JM, Martens MJ, Lemmens SG, Goebel R, Westerterp-Plantenga MS. Protein v. carbohydrate intake differentially affects liking- and wanting-related brain signalling. The British journal of nutrition. 2013;109(2):376-381.

21. Cooper AR, Goodman A, Page AS, et al. Objectively measured physical activity and sedentary time in youth: the International children's accelerometry database (ICAD). The international journal of behavioral nutrition and physical activity. 2015;12:113.

22. Heitmann BL, Westerterp KR, Loos RJ, et al. Obesity: lessons from evolution and the environment. Obesity reviews : an official journal of the International Association for the Study of Obesity. 2012;13(10):910-922.

23. Speakman JR, Westerterp KR. Associations between energy demands, physical activity, and body composition in adult humans between 18 and $96 \mathrm{y}$ of age. The American journal of clinical nutrition. 2010;92(4):826-834.

24. Soeters MR, Soeters PB. The evolutionary benefit of insulin resistance. Clinical nutrition (Edinburgh, Scotland). 2012;31(6):1002-1007.

25. Cree-Green M, Triolo TM, Nadeau KJ. Etiology of insulin resistance in youth with type 2 diabetes. Current diabetes reports. 2013;13(1):81-88.

26. Goran MI, Shaibi GQ, Weigensberg MJ, Davis JN, Cruz ML. Deterioration of insulin sensitivity and beta-cell function in overweight Hispanic children during pubertal transition: a longitudinal assessment. Int J Pediatr Obes. 2006;1(3):139-145.

27. Xu L, Li M, Yin J, et al. Change of Body Composition and Adipokines and Their Relationship with Insulin Resistance across Pubertal Development in Obese and Nonobese Chinese Children: The BCAMS Study. International journal of endocrinology. 2012;2012:389108.

28. Aradillas-Garcia C, Rodriguez-Moran M, Garay-Sevilla ME, Malacara JM, Rascon-Pacheco RA, Guerrero-Romero F. Distribution of the homeostasis model assessment of insulin resistance in Mexican children and adolescents. European journal of endocrinology. 2012;166(2):301-306.

29. Moran A, Jacobs DR, Jr., Steinberger J, et al. Insulin resistance during puberty: results from clamp studies in 357 children. Diabetes. 1999;48(10):2039-2044.

30. Lee JM, Okumura MJ, Davis MM, Herman WH, Gurney JG. Prevalence and determinants of insulin resistance among U.S. adolescents: a population-based study. Diabetes care. 2006;29(11):24272432.

31. Goran MI, Gower BA. Longitudinal study on pubertal insulin resistance. Diabetes. 2001;50(11): 2444-2450.

32. Caserta CA, Pendino GM, Alicante $S$, et al. Body mass index, cardiovascular risk factors, and carotid intima-media thickness in a pediatric population in southern Italy. Journal of pediatric gastroenterology and nutrition. 2010;51(2):216-220.

33. Bao W, Srinivasan SR, Berenson GS. Persistent elevation of plasma insulin levels is associated with increased cardiovascular risk in children and young adults. The Bogalusa Heart Study. Circulation. 1996;93(1):54-59.

34. Dorenbos E, Rijks JM, Adam TC, Westerterp-Plantenga MS, Vreugdenhil AC. Sleep efficiency as a determinant of insulin sensitivity in overweight and obese adolescents. Diabetes, obesity \& metabolism. 2015;17 Suppl 1:90-98.

35. Dorenbos E, Drummen M, Rijks J, et al. PREVIEW: Prevention of diabetes through lifestyle intervention in a multicentre study in Europe in children (10-17y). Design, methods, and baseline results. Diabetes, obesity \& metabolism. 2018;20(5):1096-1101.

36. Lee JM. Insulin resistance in children and adolescents. Reviews in endocrine \& metabolic disorders. 2006;7(3):141-147. 
37. Levy-Marchal C, Arslanian S, Cutfield W, et al. Insulin resistance in children: consensus, perspective, and future directions. The Journal of clinical endocrinology and metabolism. 2010;95(12):5189-5198.

38. Kirk S, Zeller M, Claytor R, Santangelo M, Khoury PR, Daniels SR. The relationship of health outcomes to improvement in BMI in children and adolescents. Obesity research. 2005;13(5):876882.

39. O'Connor EA, Evans CV, Burda BU, Walsh ES, Eder M, Lozano P. Screening for Obesity and Intervention for Weight Management in Children and Adolescents: Evidence Report and Systematic Review for the US Preventive Services Task Force. Jama. 2017;317(23):2427-2444.

40. Ho M, Garnett SP, Baur LA, et al. Impact of dietary and exercise interventions on weight change and metabolic outcomes in obese children and adolescents: a systematic review and metaanalysis of randomized trials. JAMA Pediatr. 2013;167(8):759-768.

41. Chiavaroli V, Giannini C, D'Adamo E, et al. Weight loss in obese prepubertal children: the influence of insulin resistance. Endocrine research. 2013;38(1):48-57.

42. Pinhas-Hamiel O, Lerner-Geva L, Copperman N, Jacobson MS. Insulin resistance and parental obesity as predictors to response to therapeutic life style change in obese children and adolescents 10-18 years old. The Journal of adolescent health : official publication of the Society for Adolescent Medicine. 2008;43(5):437-443.

43. Travers $\mathrm{SH}$, Jeffers $\mathrm{BW}$, Eckel RH. Insulin resistance during puberty and future fat accumulation. The Journal of clinical endocrinology and metabolism. 2002;87(8):3814-3818.

44. Maffeis C, Moghetti P, Grezzani A, Clementi M, Gaudino R, Tato L. Insulin resistance and the persistence of obesity from childhood into adulthood. The Journal of clinical endocrinology and metabolism. 2002;87(1):71-76.

45. Cummings DM, Henes S, Kolasa KM, Olsson J, Collier D. Insulin resistance status: predicting weight response in overweight children. Archives of pediatrics \& adolescent medicine. 2008;162(8):764-768.

46. Cappuccio FP, Taggart FM, Kandala N-B, et al. Meta-Analysis of Short Sleep Duration and Obesity in Children and Adults. Sleep. 2008;31(5):619-626.

47. Cespedes EM, Rifas-Shiman SL, Redline S, Gillman MW, Pena MM, Taveras EM. Longitudinal associations of sleep curtailment with metabolic risk in mid-childhood. Obesity (Silver Spring, Md). 2014;22(12):2586-2592.

48. Quist JS, Sjödin A, Chaput J-P, Hjorth MF. Sleep and cardiometabolic risk in children and adolescents. Sleep Medicine Reviews. 2016;29:76-100.

49. Rutters F, Gerver WJ, Nieuwenhuizen AG, Verhoef SPM, Westerterp-Plantenga MS. Sleep duration and body-weight development during puberty in a Dutch children cohort. International Journal Of Obesity. 2010;34:1508.

50. Knutson KL. The association between pubertal status and sleep duration and quality among a nationally representative sample of U. S. adolescents. American journal of human biology : the official journal of the Human Biology Council. 2005;17(4):418-424.

51. Thorleifsdottir B, Bjornsson JK, Benediktsdottir B, Gislason T, Kristbjarnarson H. Sleep and sleep habits from childhood to young adulthood over a 10-year period. Journal of psychosomatic research. 2002;53(1):529-537.

52. Klingenberg L, Chaput JP, Holmback U, et al. Acute Sleep Restriction Reduces Insulin Sensitivity in Adolescent Boys. Sleep. 2013;36(7):1085-1090.

53. Schmid SM, Hallschmid M, Schultes B. The metabolic burden of sleep loss. The lancet Diabetes \& endocrinology. 2015;3(1):52-62.

54. Garaulet M, Ortega FB, Ruiz JR, et al. Short sleep duration is associated with increased obesity markers in European adolescents: effect of physical activity and dietary habits. The HELENA study. International journal of obesity (2005). 2011;35(10):1308-1317.

55. Buysse DJ, Reynolds CF, 3rd, Monk TH, Berman SR, Kupfer DJ. The Pittsburgh Sleep Quality Index: a new instrument for psychiatric practice and research. Psychiatry research. 1989;28(2):193-213. 
56. Khan UI, McGinn AP, Isasi CR, et al. Differences in Cardiometabolic Risk between InsulinSensitive and Insulin-Resistant Overweight and Obese Children. Childhood obesity (Print). 2015;11(3):289-296.

57. AlKhater SA. Paediatric non-alcoholic fatty liver disease: an overview. Obesity reviews : an official journal of the International Association for the Study of Obesity. 2015;16(5):393-405.

58. Bush H, Golabi P, Younossi ZM. Pediatric Non-Alcoholic Fatty Liver Disease. Children (Basel, Switzerland). 2017;4(6):48.

59. Jimenez-Rivera C, Hadjiyannakis S, Davila J, et al. Prevalence and risk factors for non-alcoholic fatty liver in children and youth with obesity. BMC Pediatrics. 2017;17(1):113.

60. Ozturk Y, Soylu OB. Fatty liver in childhood. World journal of hepatology. 2014;6(1):33-40.

61. Gruben N, Shiri-Sverdlov R, Koonen DP, Hofker MH. Nonalcoholic fatty liver disease: A main driver of insulin resistance or a dangerous liaison? Biochimica et biophysica acta. 2014;1842(11):23292343.

62. Lonardo A, Ballestri S, Marchesini G, Angulo P, Loria P. Nonalcoholic fatty liver disease: a precursor of the metabolic syndrome. Digestive and liver disease : official journal of the Italian Society of Gastroenterology and the Italian Association for the Study of the Liver. 2015;47(3):181190.

63. Valenti L, Bugianesi E, Pajvani U, Targher G. Nonalcoholic fatty liver disease: cause or consequence of type 2 diabetes? Liver international : official journal of the International Association for the Study of the Liver. 2016;36(11):1563-1579.

64. Schwimmer JB, Dunn W, Norman GJ, et al. SAFETY study: Alanine aminotransferase cutoff values are set too high for reliable detection of pediatric chronic liver disease. Gastroenterology. 2010;138(4):1357-1364.e1352.

65. Yu EL, Golshan S, Harlow KE, et al. Prevalence of Nonalcoholic Fatty Liver Disease in Children with Obesity. The Journal of pediatrics. 2018. 


\title{
Appendices
}

\author{
Summary \\ Samenvatting \\ Valorisation \\ Dankwoord \\ About the author \\ List of publications
}




\section{Summary}

During puberty a physiological, transient increase in insulin resistance (IR) occurs, which might ultimately lead to hyperglycaemia and type 2 diabetes mellitus (T2DM). IR is a comorbidity of obesity and the level of IR appeared to increase with the level of obesity, with up to $52 \%$ of adolescents having IR. Moreover, adolescents can convert from IR to T2DM in as little as 21 months. Prevention of further increase of $I R$, especially in adolescents with overweight/obesity or other risk factors, might therefore decrease risk for T2DM development.

This thesis aimed to gain more insight in determinants of pubertal insulin resistance, especially in adolescents with overweight/obesity at risk for T2DM development. In addition, the effects of a lifestyle intervention on IR in insulin resistant adolescents with overweight/ obesity are presented. In 137 adolescents with overweight and obesity from the Centre for Overweight Adolescent and Children's Healthcare (COACH) associations of BMI z-score, pubertal stage, age and physical activity with IR were analysed (CHAPTER 2). In addition, the data of the PREVention of diabetes through lifestyle Intervention and population studies in Europe and around the World (PREVIEW) study in adolescents is presented. As part of the larger international PREVIEW randomized controlled trial, the PREVIEW study in adolescents aimed to assess the effects of a high-protein low-glycaemic index (Gl) diet, compared to a moderate-protein moderate-GI diet, on IR and BMI z-score in adolescents with overweight/obesity and at high risk for developing T2DM. It was hypothesized that the HP diet would be superior in reducing IR in insulin resistant adolescents with overweight/ obesity, compared to the MP diet. 126 adolescents from the Netherlands, Spain and United Kingdom that had overweight or obesity and high IR were randomized into a high-protein low-GI (HP, 25En\%) or moderate-protein moderate-GI (MP, 15En\%) group (CHAPTER 3). In addition, all participants received instructions to increase physical activity (PA). At baseline, after one year and two years of intervention IR, BMI z-score, and cardiometabolic parameters were measured. Furthermore, lifestyle variables such as reported dietary intake, food intake behaviour, PA and sleep characteristics were measured and correlated with (changes in) IR and BMI z-score.

Homeostatic Model Assessment of Insulin Resistance (HOMA-IR) was significantly higher in postpubertal boys compared to prepubertal boys, which is a confirmation of earlier studies (CHAPTER 2). In girls a similar, although not significant, trend was observed. In the PREVIEW study, too, postpubertal adolescents with morbid obesity showed significantly higher HOMA-IR compared to prepubertal subjects with morbid obesity, or overweight/obese 
subjects at any pubertal stage (CHAPTER 3). This adds to current evidence indicating that especially adolescents with higher obesity status have high IR at the end of puberty.

Both studies observed a positive relationship between BMI z-score and HOMA-IR, reaffirming current literature that higher BMI z-score, and thus higher obesity status, was related to higher IR. In the study of $\mathrm{COACH}$ participants a direct positive association between BMI z-score and HOMA-IR was observed in girls, while in boys there was a trend for this relationship (CHAPTER 2). However, after correcting for age, puberty stage and self-reported physical activity, BMI z-score and HOMA-IR were positively associated in boys, too. In the PREVIEW study the group was divided according to pubertal stage and obesity status. Markers of adiposity (BMI z-score, but also fat free mass index, fat mass index and fat percentage) were positively related to HOMA-IR (CHAPTER 3). Thus, adolescents with overweight/obesity are at higher risk of $\beta$-cell exhaustion and T2DM development, which requires optimization of therapeutic strategies for prevention of further IR increase.

Changes in reported protein intake were not significally different between at any timepoint, nor between the HP and MP intervention groups (CHAPTER 4). In addition, a drop-out rate of $34 \%$ after one year and $61 \%$ after two years of intervention was observed. As a possible consequence the groups did not significantly differ in changes in BMI z-score change, IR nor other cardiometabolic parameters. Maintaining the relatively high protein target of $25 \mathrm{En} \%$ in the HP group was obviously not feasible during extended periods of time with instructions alone. Achieving and maintaining a target of $25 \mathrm{En} \%$ protein might only be feasible with vouchers or subsidies for foods high in protein, the use of protein supplements or meal replacements.

Nonetheless, after one year of PREVIEW intervention HOMA-IR stabilized despite progression in pubertal stage. Change in HOMA-IR was positively related to change in BMI z-score after one year, indicating that as adolescents reduced their BMI z-score they also reduced IR. Moreover, after one and two years of PREVIEW intervention a significant and clinically relevant BMI z-score reduction was observed in the total group. The change in BMI z-score was not attributable to one of the dietary strategies of the PREVIEW study. Change in $\mathrm{BMI} z$-score was however inversely related to change in cognitive restraint of eating scores and positively to hunger scores on the Three Factor Eating Questionnaire (TFEQ). As the dietary restraint scores increased significantly after one and two years, this indicated that as adolescents changed their attitude towards food intake they decreased their BMI z-score. The reduction in BMI z-score was counteracted by an increase in hunger scores.

In the cross-sectional COACH study self-reported physical activity, as reported in the Baecke questionnaire, was inversely related to HOMA-IR in boys (but not in girls) after correction for age, BMI z-score and puberty stage (CHAPTER 2). Baseline data of the PREVIEW study, too, showed that Baecke Sport scores were inversely related to glucose concentration, after correction for sex, pubertal stage, BMI z-score and fat mass percentage (CHAPTER 3). 
After one and two years of PREVIEW participation accelerometry counts and minutes spent in moderate-to-vigorous physical activity increased significantly while sedentary time decreased, indicating compliance to the physical activity guidelines (CHAPTER 4). However, no direct, independent relationships between PA with IR or BMI z-score were observed.

This thesis also aimed to explore associations of sleep characteristics and non-alcoholic fatty liver disease on (intervention-related changes in) BMI z-score and IR. Sleep characteristics, and especially short sleep duration and sleep debt, have been related to increased risk of developing obesity and IR in childhood. In the COACH study, total sleeping time and sleep efficiency (total sleeping time as a percentage of time spent in bed, measured by polysomnography) were inversely related to HOMA-IR, indicating that longer sleep duration and more quality sleep were related to less IR (CHAPTER 2). This observation was only found in prepubertal girls, and not in boys or girls that had already entered pubertal development. No direct associations between sleep duration or sleep architecture parameters and HOMAIR were observed in the PREVIEW study. We observed that habitual (actigraph-measured) sleep, but not polysomnography-measured sleep, was negatively related to BMI z-score at the onset of the study. While mean BMI z-score decreased significally after 1 year of study participation, sleep parameters did not change and were not associated with changes in anthropometric and cardiometabolic risk variables (CHAPTER 5). The relatively larger decrease in BMI z-score may have counteracted possible effects of sleep characteristics on HOMA-IR.

Non-alcoholic fatty liver disease (NAFLD) has been associated with overweight/obesity and IR in adolescence. We assessed the role of NAFLD on changes in BMI z-score and HOMAIR during one year of PREVIEW intervention. The $32.5 \%$ of adolescents that had increased alanine aminotransferase (ALT) concentration at baseline, indicating NAFLD, showed significantly less BMI z-score reduction after one year intervention than the adolescents that had no signs of NAFLD (CHAPTER 6). ALT concentration was also positively related to HOMAIR at study onset. These results indicated that the presence of increased ALT concentrations negatively affected intervention outcomes in addition to predisposing to IR.

In conclusion, this thesis aimed to gain insight in determinants of pubertal insulin resistance in adolescents with overweight/obesity, as well as presenting the effects of the PREVIEW lifestyle intervention on IR and BMI z-score in adolescents with overweight/obesity at high risk for T2DM development. Achieving and maintaining a high protein intake, as was recommended by the PREVIEW study, was not feasible and no effects of a HP diet on IR in adolescents with overweight/obesity were observed. We confirmed earlier studies that BMI z-score was positively related to HOMA-IR and showed that HOMA-IR stabilization after one year of intervention could be achieved by BMI z-score reduction. BMI z-score reduction was accomplished by increased dietary restraint in participants, which significantly increased 
after dietary guidance. BMI z-score was not independently associated to change in those lifestyle variables nor to (change in) sleep characteristics. NAFLD was observed in $1 / 3^{\text {rd }}$ of participants and was associated with significantly less BMI z-score during intervention. We would therefore recommend to put emphasis on increasing dietary restraint in therapeutic strategies, as this was related to decreased BMI z-score and IR stabilization in adolescents with overweight/obesity at high risk for T2DM development. Furthermore, we recommend to screen for the presence of NAFLD as this might counteract BMI z-score reduction. 


\section{Samenvatting}

Tijdens de puberteit vindt er tijdelijk een fysiologische insulineresistentie (IR) plaats, die halverwege de puberteit piekt en aan het eindevan de puberteit weer afneemt. Deverhoogde glucosespiegels in het bloed worden dan gecompenseerd door insulineproductie in de $\beta$-cel van de pancreas. IR is een comorbiditeit van obesitas en de mate van IR lijkt samen te hangen met de mate van obesitas. Tot $52 \%$ van de adolescenten met overgewicht of obesitas hebben een verhoogde IR. Bij hen is het met name van belang te voorkomen dat IR verder toeneemt tijdens de puberteit om het risico op het ontstaan van type 2 diabetes mellitus (T2DM) te verminderen.

Het doel van de studies beschreven in dit proefschrift was meer inzicht te krijgen in determinanten van insulineresistentie tijdens de puberteit. Het effect van een leefstijlinterventie op IR en leeftijds- en geslacht gecorrigeerde BMI z-score werd onderzocht bij adolescenten met overgewicht of obesitas en insulineresistentie. Bij 137 adolescenten met overgewicht of obesitas van het Centre for Overweight Adolescent and Children's Healthcare (COACH) werd het verband tussen IR en BMI z-score, puberteitsstadium, leeftijd en fysieke activiteit onderzocht (HOOFDSTUK 2). De PREVIEW studie bij adolescenten (PREVention of diabetes through lifestyle Intervention and population studies in Europe and around the World (PREVIEW) onderzocht het effect van een hoog-eiwit lage-glycemische index (GI) dieet, versus een medium-eiwit medium-GI dieet, op IR en BMI z-score. De hypothese was dat een hogere eiwitinname gunstiger zou zijn voor verlaging van de insulineresistentie en BMI z-score (HOOFDSTUK 3). 126 adolescenten met overgewicht of obesitas en IR uit Nederland, Spanje en het Verenigd Koninkrijk werden gerandomiseerd in één groep die advies kreeg om een hoogeiwit laag-GI dieet te volgen (HP, 25En\% eiwit), en één groep die instructies kreeg voor een medium-eiwit medium-GI dieet (MP, 15En\%). Hiernaast werden deelnemers geïnstrueerd om hun fysieke activiteit te verhogen. Aan het begin van de studie, na één en na twee jaar leefstijlinterventie werden BMI z-score, IR en andere cardiometabole parameters gemeten. Hiernaast werden leefstijlfactoren zoals gerapporteerde voedselinname, voedselinname gedrag, fysieke activiteit en slaap parameters bepaald, en gecorreleerd met IR, BMI z-score en veranderingen hierin.

Bij jongens aan het einde van de puberteit bleek de insuline resistentie (Homeostatic Model Assessment of Insulin Resistance (HOMA-IR)) significant hoger te zijn dan bij jongens aan het begin van de puberteit (HOOFDSTUK 2). Bij meisjes werd een vergelijkbare trend gezien. Uit de PREVIEW studie bleek ook dat adolescenten met morbide obesitas aan het einde van de puberteit een hogere HOMA-IR hadden dan adolescenten met morbide obesitas aan 
het begin van de puberteit; tevens was deze gedurende de hele puberteit hoger dan bij adolescenten met overgewicht of obesitas (HOOFDSTUK 3). Deze resultaten zijn in lijn met eerdere wetenschappelijke observaties, en lijken erop te wijzen dat vooral adolescenten met een hogere obesitasstatus ook aan het einde van de puberteit een hoge IR hebben.

In beide studies waren BMI z-score en HOMA-IR positief gecorreleerd: een hogere IR hing dus samen met hogere BMI z-score. In de $\mathrm{COACH}$ studie bleek er bij meisjes een directe relatie tussen $\mathrm{BMI}$ z-score en HOMA-IR te zijn, bij jongens was deze aanwezig na correctie voor leeftijd, puberteitsstadium en gerapporteerde fysieke activiteit. In de PREVIEW studie werden de analyses gestratificeerd op basis van puberteitsstadium en obesitasgraad. Ook hier bleek dat obesitas (BMI z-score, maar ook vetvrije massa index, vetmassa index en vetpercentage) positief gerelateerd waren aan HOMA-IR (HOOFDSTUK 3). Samengevat kan worden geconcludeerd dat adolescenten met overgewicht en obesitas een hoger risico lijken te hebben op $\beta$-cel uitputting en ontwikkeling van T2DM. Deze observaties benadrukken het belang van optimalisering van therapeutische strategieën om een verdere toename van IR te voorkomen.

Gedurende de twee jaar aangeboden leefstijlinterventie bleken er noch tussen de verschillende meetmomenten, noch tussen de HP en MP groep, significante verschillen in gerapporteerde eiwitinname te zijn (HOOFDSTUK 4). Hiernaast bleek na één jaar 34\% en na twee jaar $61 \%$ van de deelnemers uitgevallen te zijn. Als consequentie hiervan werden er geen verschillen gevonden tussen de HP en MP groep met betrekking tot veranderingen in BMI z-score, IR en andere cardiometabole parameters. Deze resultaten wezen erop dat het behalen en volhouden van een verhoogde eiwitinname gedurende lange tijd niet haalbaar was met enkel instructies. Mogelijk zou dit alleen haalbaar geweest zijn met vouchers of subsidies voor eiwitrijke producten, maaltijdvervangers of het gebruik van eiwitsupplementen.

Desondanks bleek dat na één jaar PREVIEW leefstijlinterventie HOMA-IR gestabiliseerd was in de gehele groep, wat een indicatie vormde voor het enigszins beperken van toename van IR gedurende de puberteit. Verandering in HOMA-IR was positief geassocieerd met verandering in BMI z-score na één jaar, wat erop duidde dat afname in IR samenhing met afname van de BMI z-score. Na één en twee jaar interventie was de BMI z-score in de gehele groep significant gedaald. Deze verandering in BMI z-score was niet toe te schrijven aan verandering in één van de leefstijlfactoren. Wel scoorden de deelnemers na één jaar hoger op cognitief beheerst eetgedrag, wat geassocieerd was met de daling van de BMI z-score.

In de $\mathrm{COACH}$ studie bleek gerapporteerde fysieke activiteit op de Baecke vragenlijst omgekeerd gerelateerd te zijn aan IR bij jongens na correctie voor leeftijd, BMI z-score en puberteitsstadium (HOOFDSTUK 2). Ook bij de PREVIEW studie waren gerapporteerde Baecke Sportscores gerelateerd aan lagere bloedglucoseconcentratie na correctie voor geslacht, puberteitsstadium, BMI z-score en vetpercentage (HOOFDSTUK 3). Na één en 
twee jaar PREVIEW leefstijlinterventie bleken ook de objectief gemeten matig-tot-intensieve fysieke activiteit te zijn toegenomen, terwijl sedentaire activiteit verminderd was (HOOFDSTUK 4). De instructies om de fysieke activiteit te verhogen werden dus opgevolgd; echter dit was niet geassocieerd met de verandering in IR of BMI z-score.

Tevens werden in dit proefschrift mogelijke associaties van slaap kenmerken en van nietalcoholische leververvetting met IR en BMI z-score (en interventie-gerelateerde veranderingen hierin) onderzocht. Een korte slaapduur werd in eerdere onderzoek in verband gebracht met een verhoogde kans op het ontwikkelen van IR en obesitas bij kinderen. In de $\mathrm{COACH}$ studie bleek dat totale slaaptijd en slaapefficiëntie omgekeerd gerelateerd waren aan HOMA-IR (HOOFDSTUK 2). Dit verband werd echter alleen gevonden bij meisjes aan het begin van de puberteit, en niet bij jongens en meisjes die verder in de puberteit waren. In de PREVIEW studie werd geen direct verband gevonden tussen slaapduur of slaaparchitectuur parameters en HOMA-IR. Bij aanvang van de studie bleek de gewoonlijke slaapduur omgekeerd gerelateerd te zijn aan de BMI z-score. Dit was niet het geval wanneer de slaapduur in de kliniek werd bepaald. Na één jaar PREVIEW interventie waren de slaapparameters niet significant veranderd, en associaties met de BMI z-score of andere variabelen was afwezig (HOOFDSTUK 5). Hierbij speelde waarschijnlijk daling van de BMI z-score een rol.

Niet-alcoholische leververvetting (NAFLD) is in eerdere studies gerelateerd aan overgewicht/obesitas en IR tijdens de puberteit. In een van de studies beschreven in dit proefschrift werd de rol van NAFLD op veranderingen in BMI z-score en IR tijdens de PREVIEW leefstijlinterventie onderzocht. 32.5\% van de adolescenten had bij aanvang van de studie verhoogde alanine aminotransferase (ALT) concentraties, indicatief voor de aanwezigheid van NAFLD. Deze adolescenten lieten een significant mindere afname van de BMI z-score na één jaar interventie zien, in vergelijking tot de adolescenten die geen aanwijzingen voor NAFLD hadden (HOOFDSTUK 6). Bij aanvang van de studie bleek de ALT concentratie tevens positief geassocieerd te zijn met HOMA-IR. Deze resultaten wijzen erop dat verhoogde ALT concentraties, suggestief voor NAFLD, de uitkomsten van de leefstijlinterventie negatief beïnvloedden en hiernaast geassocieerd waren met verhoogde IR.

Samengevat was het doel van de studies beschreven in dit proefschrift inzicht te krijgen in determinanten van insulineresistentie. Het effect van een leefstijlinterventie op IR en BMI z-score werd onderzocht bij adolescenten met overgewicht/obesitas en IR. De gerandomiseerde, gecontroleerde klinische PREVIEW studie bij adolescenten bleek niet haalbaar te zijn, vanwege de uitval van deelnemers en daardoor gemis aan meetresultaten. Tevens werd er geen verandering en geen verschil in de eiwitinname van de twee dieetgroepen waargenomen. Daardoor ontstonden er geen verschillen in HOMA-IR, noch in verandering in BMI z-score, tussen de groepen. De waargenomen daling in BMI 
z-score was geassocieerd met een significante toename in cognitief beheerst eetgedrag. HOMA-IR stabiliseerde na één jaar, en was geassocieerd met de afname in BMI z-score. Verandering in de BMI z-score was niet gerelateerd aan verandering in leefstijlvariabelen of in slaapparameters. Aanwijzingen voor NAFLD bleek een risicofactor te zijn voor een significant verminderde afname in BMI z-score tijdens de leefstijlinterventie. Op basis van het onderzoek gepresenteerd in dit proefschrift wordt geadviseerd om bij de behandeling van adolescenten met overgewicht/obesitas en verhoogd risico op T2DM cognitief beheerst eetgedrag te stimuleren. Hiernaast adviseren wij om vóór aanvang van de behandeling te screenen op NAFLD omdat dit een risicofactor bleek voor minder BMI z-score afname. 


\section{Valorisation}

A recent report estimated that, without proven effective interventions, in 2025 a total of 268 million children will be overweight, of which 91 million will have obesity. Even in childhood obesity is already associated with a myriad of comorbidities affecting nearly every organ system, including but not limited to type 2 diabetes mellitus (T2DM), non-alcoholic fatty liver disease (NAFLD), as well as psychological consequences such as reduced quality of life. This thesis particularly focusses on the prevention of T2DM in adolescents. T2DM was typically considered to be an adult-onset disease, but in recent years $45 \%$ of new diabetes cases in adolescents were attributable to type 2 . Moreover, up to $52 \%$ of adolescents with overweight present with IR, a known precursor of T2DM, and it is known that adolescents can convert from IR to T2DM much faster than adults. It is therefore important to gain more insight in IR in adolescence - and that research in this field is relevant, available and can be used for translation so that new strategies to prevent T2DM in youth can be developed.

The PREVIEW study was designed to assess the feasibility and effects of a lifestyle intervention, consisting of increasing protein intake, on IR and BMI z-score in adolescents with overweight and obesity at high risk for developing T2DM. Adolescents (aged 10-17y) with overweight/ obesity and IR from the Netherlands, Spain and United Kingdom were randomized into two groups: a high-protein low-glycaemic-index (HP) group that was advised to increase protein intake to $25 \%$ of daily energy intake (En\%), and a moderate-protein moderate-glycaemicindex (MP) group that increased protein to $15 \mathrm{En} \%$. Adolescents were also encouraged to increase physical activity. The second aim of the PREVIEW study was to gain more knowledge about the relationship with possible modifiable risk factors, specifically sleep duration and architecture and NAFLD, with pubertal IR and intervention-mediated changes herein.

Previous studies have shown that adolescents that have overweight/obesity and are also insulin resistant, are at risk for less or no BMI z-score reduction during lifestyle intervention compared to adolescents with overweight/obesity but without IR. In the PREVIEW study only adolescents with overweight/obesity and IR were selected, and mean BMI z-score decreased with -0.17 SD after one year and -0.19 SD after two years of intervention. This reduction is not only statistically significant, but also clinically relevant as BMI z-score reductions >-0.15 have been related to improvement in glucose metabolism and cardiovascular risk markers. This shows that the advice to increase protein intake (in combination with instructions to increase physical activity) and personalized approach of the PREVIEW study are targets for successful treatment of adolescents with overweight/obesity - which is especially beneficial for those with IR as that were until now at risk for less intervention response. 
Furthermore, the personalized instructions of the PREVIEW study rendered an overall increase in physical activity, especially moderate-to-vigorous activity and decrease in sedentary time, suggesting favourable changes in lifestyle parameters. After one year cognitive restraint of eating scores were significantly higher - indicating a more conscious attitude towards food intake - and this was directly associated with BMI z-score decrease. Thus, the results of this study indicated that increasing dietary restraint is a target for successful BMI z-score reduction in adolescents with overweight and obesity, which might be of benefit for patients as well as for clinicians and scientists aiming to improve current childhood obesity therapies.

It was also observed that NAFLD, which was present in $1 / 3^{\text {rd }}$ of the adolescents in this cohort, was a risk factor for less BMI z-score decrease after one year of intervention. As screening for NAFLD at treatment onset is a relatively easy, cheap and non-invasive procedure, we would recommend to incorporate this in treatment strategies as this will effectively identify individuals that might be in need of additional support to achieve successful BMI z-score decrease.

None of the adolescents progressed to T2DM during the two years of study participation. After one year IR stabilization could be achieved despite progression of puberty, and this was related to decrease in BMI z-score. After two years HOMA-IR increased, suggesting that adolescents with overweight and obesity are at risk for persistence of high IR at the end of puberty, which is in line with cross-sectional observations from this and other studies. Thus, these results indicate the importance of preventing further IR exacerbation during puberty, especially in those with overweight/obesity as they are at risk for persistent high $\mathrm{IR}$, but also demonstrate that it is possible to counteract IR increase and T2DM progression by decreasing BMI z-score. The results of the PREVIEW study are relevant to the society, because it provides a novel therapeutic strategy that successfully prevented IR increase and yielded BMI z-score decrease in a population that was previously at risk for less successful therapy outcomes. The PREVIEW study also identified several targets, e.g. increasing cognitive restraint of eating and screening for NAFLD, that are associated with BMI z-score decrease after one year of intervention and might thus aid clinicians and scientists aiming to improve current childhood obesity therapies. These results were shared with the scientific community and presented at several national and international conferences.

Apart from the medical and societal relevance, the outcomes of this study also have an economical relevance. The costs of diabetes are rising rapidly and are estimated to be a $\$ 327$ mil per year in the USA alone. For children it has been shown that overweight and obesity lead to an incremental lifetime health costs of $\$ 19.000$,- per individual (data of 2012), resulting in considerable economical burden considering the estimated number of T2DM 
globally as reported at the beginning of this chapter. At this moment a cost-benefit analysis of PREVIEW is being performed. A similar lifestyle intervention in children with overweight and obesity estimated a lifetime economic benefit of $€ 11384$,- to $€ 19120$,- per individual, and estimated that the economic benefit of an intervention in childhood were 4 to 7 times higher than the costs. As the PREVIEW study was particularly successful in those adolescents with high risk for T2DM development (and thus higher lifetime healthcare costs), it is expected that this lifestyle intervention contributes to reduced healthcare costs for the treatment of childhood obesity and diabetes.

Not only positive research results are valuable for the scientific and medical community. PREVIEW adds to a limited number of studies that aimed to increase protein intake in adolescents with overweight and obesity, and similar to our study most other studies observed that higher protein intake targets could not be achieved and maintained longterm in free living settings. However, when higher protein targets were met (as was the case in studies with an in-centre or in-centre-supermarket design) this resulted in significant BMI z-score decrease and reduction in HOMA-IR. These results indicate that achieving high protein targets with advice alone were not feasible in adolescents in free living settings, and future studies assessing the benefits of high-protein diets in adolescents should consider the incorporation of meal replacement, vouchers for protein-rich meals or protein supplements in their design.

Taken together, the PREVIEW intervention yielded a significant BMI z-score decrease in adolescents with overweight/obesity and IR, that were until now known to show significantly less BMI z-score decrease in conventional therapeutic strategies compared to peers without IR. In this group, that is at particularly high risk of developing T2DM, IR stabilized after one year of treatment which was associated to the reduction in BMI z-score. Increased dietary restraint scores were identified as targets for BMI z-score decrease, while NAFLD at study onset was related to less BMI $z$-score decrease after one year of study participation. A major strength of the study is that it was set in real-life settings, and the principles used in the PREVIEW study can therefore be very easily incorporated in other lifestyle interventions for adolescents with overweight and obesity. 


\section{Dankwoord}

Hier is het resultaat van jarenlange inzet van mij én steun van jullie: mijn promotieboekje. Wat ben ik er trots op!

Een promotie doe je nooit alleen. Daarom wil ik graag de vele mensen bedanken die me de afgelopen jaren geholpen hebben bij dit proces.

Ik kan natuurlijk niet anders dan beginnen bij mijn promotieteam: prof. dr. Margriet Westerterp-Plantenga, dr. Anita Vreugdenhil, en dr. Tanja Adam. Beste Anita, in 2014 kwam ik als student bij COACH en het duurde niet lang voor jij mij vroeg om PREVIEW en het bijbehorende promotietraject over te nemen. Van het begin af aan hebben wij dit samen opgepakt. Bedankt voor je vertrouwen, doorzettingsvermogen en levensadviezen - "fake it till you become it" blijkt ook ver buiten het promotieleven erg nuttig te zijn ;-) Margriet, dank voor de mogelijkheid om als uw laatste PhD student te mogen promoveren en het vertrouwen om op diverse (inter)nationale congressen de studieresultaten te mogen delen. Tanja, dank voor de begeleiding en dat jouw deur altijd open stond voor een praatje.

Tevens wil ik graag de leden van de leescommissie: prof.dr. Edward Dompeling, prof. dr. Edith Feskens, prof. dr. Hanno Pijl, prof. dr. Ronit Sverdlov en prof. dr. Edgar van Mil bedanken voor de beoordeling van en feedback op deze thesis.

To all the members of the PREVIEW consortium, thank you for all your input on the many versions of the manuscripts and presentations. Hierbij wil ik Mathijs in het bijzonder bedanken. Ondanks dat de studie er voor ons totaal verschillend uit zag, heb je ontzettend veel voor de kinderstudie betekend en was het voor mij heel waardevol om met je te kunnen sparren over zowel de inhoud als het promoveren zelf.

Bjorn Winkens, wat ben ik blij dat jij aansloot bij het werken aan de papers als mijn GPS ("grudgingly preferred statistician" - zoals je jezelf ooit noemde). Met jouw hulp, steun en eindeloze geduld hebben we menig voorheen onoplosbaar statistisch dilemma kunnen oplossen. Ook je relativerende en humoristische kijk op de wetenschap heb ik al deze tijd erg gewaardeerd.

Loe Donselaar, ik heb regelmatig een middagje bij je mogen pipetteren en nog vaker samples langsgebracht voor de diverse studies. Dank voor al je hulp bij de analyses en mijn vragen hierover. 
Aan alle lieve collega's van COACH: wat zijn jullie fijn! Vanaf het moment dat ik me bij jullie team mocht voegen, waren jullie als een warm bad. Ik heb ontzettend genoten van de jaren die we samengewerkt hebben. Nog iedere keer verwonder ik mij over de vele prachtige initiatieven en de manier waarop iedereen zo hard werkt om het leven van kinderen met overgewicht en obesitas gezonder, maar ook zo veel leuker te maken. Jullie maken het verschil!

Uiteraard begon en eindigde mijn promotie onderzoek bij de kinderen: mijn doel is altijd geweest om iets bij te dragen voor betere zorg voor kinderen, nu en in de toekomst. Daarom wil ik in het bijzonder de kinderen en hun ouders die meededen aan PREVIEW en $\mathrm{COACH}$ bedanken voor hun vertrouwen en de manier waarop zij zo ontzettend hard hebben gewerkt.

Bij de kindergeneeskunde was het altijd gezellig in de promovendi club. Lieve Bas, Britt v E., Britt D., Dillys, Dorien, Eduardo, Gabriëlle, Jesse, Johanna, Kylie, Lisanne, Maartje, Mark, Marlou, Minela, Nadine en Yvon: van velen van jullie heb ik al een boekje in de kast staan en er zullen er nog vele volgen. Het was ontzettend gezellig om samen met jullie hard te werken, maar zo mogelijk nog meer lol te maken. Ik wens jullie allemaal veel succes in de toekomst.

Danilo, jij hebt mij geholpen om mijn eerste schreden binnen de kindergeneeskunde te zetten. Dank voor je steun en fijne gesprekken al deze jaren!

A special thanks to the Galactosemia Girls Estela, Ana, Britt, Inge, Britt and Minela: you have adopted me as one of your group and I have truly enjoyed all of our "coffee/cake/wine marathons".

Kim, het laatste jaar van mijn PhD heb jij als mijn mentor gefungeerd. Wat heb ik veel van je geleerd, zowel op wetenschappelijk als op persoonlijk gebied. Je luisterend oor, drive om het altijd nóg beter te doen, goede adviezen en moed om ook moeilijke keuzes te durven maken hebben me altijd geïnspireerd. Bedankt!

Aan alle lieve collega's van de JGZ: dank voor jullie niet aflatende steun en enthousiasme over dit promotietraject.

Britt, jou als vriendin hebben is één van de beste dingen die uit dit promotietraject is voortgekomen :-) Je was en bent nog steeds een voorbeeld voor me. Op naar nog onwijs veel gezelligheid samen in de toekomst!

Ruth, een paar jaar geleden sprak je de onsterfelijke woorden "we slepen, huilen, chocola- en koffie-nuttigen ons er wel doorheen" - en alleen de koffiedame weet hoe gelijk je had ;-). Zowel in Maastricht als in Veldhoven was je mijn buddy en heb je me onwijs kunnen laten lachen om alle (promotie en andere) perikelen waar we in verzeild raakten. Dankjewel! 
Karin, al 10 jaar lang (!) studeren, dokteren en promoveren we samen - en wat is het fijn om dat allemaal met jou te kunnen doen. Ik kijk nu al uit naar jouw promotie en de mooie tijd die we ook daarna samen kunnen delen :-)

Gabriëlle - als een wervelwind kwam je mijn kantoor (en leven) in en sindsdien ben je mijn cabaretier, relativerend vermogen, luisterend oor, motivator en vooral vriendin. Dank voor vriendinnen zoals jij!

Davy, we liepen de deur bij elkaar plat en ondanks dat de fysieke afstand nu groter is dan voorheen (ik vind nog steeds dat we weer buren moeten worden!) ben je er altijd voor me. Dankjewel!

Lex, ook jij bent ondertussen letterlijk aan het andere eind van het land gesetteld, maar wat is het fijn om alle hoogtepunten toch altijd samen te kunnen vieren.

Lieve Bauke, Camille, Pauline en Yasmin: als overactieve geneeskunde studenten wilden wij "iets leuks" doen. Dat heeft geresulteerd in duizenden genezen teddyberen, geweldige projecten die we hebben opgezet op de kinderafdeling, tig wijnavondjes en vooral héél veel gezelligheid. Wat mooi om te zien hoe ieder van ons nu haar eigen weg gaat, maar we desondanks nog steeds zulke goede vriendinnen zijn.

Lieve Corien, Daniëlle en Mirjam: ik ben nog steeds onder de indruk van jullie onwijs goede proefschriften en hopelijk past dit proefschrift mooi in de rij. Corien, succes met de laatste loodjes.

Elly, jij valt ondertussen meer in de categorie familie dan collega's of vrienden. Jouw warmte en goede adviezen hebben ontzettend veel voor mij betekend en makten al die lunches, wandelingen en keukentafelgesprekken die we mochten delen vaak het hoogtepunt van mijn week. Dankjewel voor alles!

Monique en Linda, ik heb héél lang geprobeerd om de juiste woorden te vinden die ons beschrijven maar eigenlijk zijn die er niet;-) Wat ben ik blij dat wij al meer dan een half leven alle hoogte- en dieptepunten samen kunnen meemaken, er samen om kunnen lachen, en dat jullie er altijd zijn. Met jullie is alles leuker :-)

Lieve Suzanne, José, Jerôme, hoe vaak ben ik wel niet op mijn fietsje naar Geldermalsen getrokken? Nog steeds voelen jullie een beetje als mijn adoptiefamilie. Wat fijn dat jullie er altijd voor me zijn. Su: heel veel succes met de allerlaatste loodjes!

Aan alle Dorenbosjes/Heijnsdijkjes: wat ben ik blij met een familie zoals jullie. Ondanks dat het hele promotietraject en academische wereldje soms compleet onbegrijpelijk leken, hebben jullie me altijd in alles gesteund. Fijn om nu met jullie deze mijlpaal te kunnen vieren. Lieve oma, helaas kan je er vandaag niet bij zijn maar in gedachten zit je op de eerste rij. Wat zal het fijn zijn als we elkaar binnenkort weer in de armen mogen sluiten en dit samen 
kunnen vieren. Ik hou van je!

Liebe Gerti, Andreas und Christina, was für ein Glück dass ihr so ein wichtiger Teil meiner Familie geworden seid. Schon seit dem ersten Mal wo wir uns kennen gelernt haben und wir uns nur mit einem Wörterbuch verständigen konnten, habe ich mich trotzdem direkt sehr willkommen gefühlt. Wie schön, dass ihr auch heute dabei seid.

Liefste pappa en mamma: er is werkelijk niets wat ik hier zou kunnen schrijven wat eer doet aan wat jullie voor mij betekenen. Ik ben ontzettend trots op en dankbaar voor een gezin als het onze. Jullie hebben Marlies en mij altijd gestimuleerd om het beste uit onszelf te halen en zijn daar zelf altijd het beste voorbeeld in geweest. Zonder jullie was dit boekje er niet geweest. Ik hou heel, heel veel van jullie. Lieve Marlies, je bent de leukste, grappigste, fijnste, stoerste en liefste zus die ik me kan wensen en ik ben trots op je! Je hebt ieder stapje (en koffer-incident) van het promoveren achter, voor en naast mij gestaan, en ik ben ontzettend blij dat jij ook vandaag letterlijk naast mij wil staan als paranimf :-)

Matthias, al bijna 10 jaar lang ben je mijn anker en degene die me de moed geeft om mijn dromen achterna te jagen. Jij maakt mijn leven zoveel mooier. Ik hou van je! 


\section{About the author}

Elke Dorenbos was born on July 20, 1989 in Steinheim, Germany. She graduated from her high school Lek en Linge in Culemborg in 2007, which she combined in the latter years with the Pre-University College at Leiden University. After obtaining her bachelor degree in Biomedical Sciences from Utrecht University, she was selected for the Medicine \& Clinical Research (A-KO) Master at Maastricht University, from which she graduated in 2014. She performed her final internships at the Pediatric Intensive Care Unit (dr. P. Leroy) and at the Centre for Overweight Adolescent and Children's Healthcare $(\mathrm{COACH}$, dr. A. Vreugdenhil). During her scientific internship

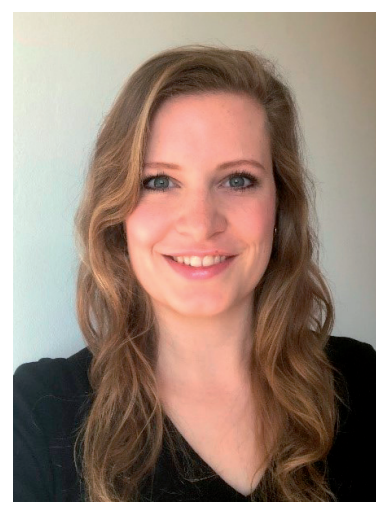
she started as a PhD student on the PREVIEW study, later combining this with working as a physician at the $\mathrm{COACH}$ clinic. Under the supervision of prof. M. Westerterp-Plantenga, dr. A. Vreugdenhil and dr. T. Adam she studied the determinants and effects of a lifestyle intervention on insulin resistance in adolescents with overweight and obesity at high risk for developing type 2 diabetes. The results of her research have been published in multiple publications and she has presented them at several national and international conferences. The most important scientific results are presented in this thesis.

During her time as a PhD student, Elke was selected for the TULIPS PhD curriculum 20172019, a two-year program for PhD students with the potential and ambition to advance their research in paediatrics as a clinician-scientist. She was also involved in the organisation of several national conferences for PhD students in paediatrics.

After working as a physician at the Department of Pediatrics and Neonatal Intensive Care Unit at Máxima Medisch Centrum in Veldhoven, she is currently working as a youth healthcare physician in Maastricht and Eijsden. She lives together with Matthias. 


\section{List of publications}

Rijks J, Plat J, Mensink R, Dorenbos E, Buurman W, Vreugdenhil A. Children With Morbid Obesity Benefit Equally as Children With Overweight and Obesity From an Ongoing Care Program. J Clin Endocrinol Metab. 2015 Sep;100(9):3572-80.

Dorenbos E, Rijks J, Adam T, Westerterp-Plantenga M, Vreugdenhil A. Sleep efficiency as a determinant of insulin sensitivity in overweight and obese adolescents. Diabetes Obes Metab. 2015 Sep;17 Suppl 1:90-8.

Rijks J, Penders B, Dorenbos E, Straetemans S, Gerver W, Vreugdenhil A. Pituitary response to thyrotropin releasing hormone in children with overweight and obesity. Scientific Reports 2016 Aug 3;6:31032.

Rijks J, Karnebeek K, van Dijk J, Dorenbos E, Gerver W, Stouthart P, Plat J, Vreugdenhil A. Glycaemic Profiles of Children With Overweight and Obesity in Free-living Conditions in Association With Cardiometabolic Risk. Scientific Reports 2016 Aug 18;6:31892.

Rijks J, Plat J, Dorenbos E, Penders B, Gerver W, Vreugdenhil A. Association of TSH With Cardiovascular Disease Risk in Overweight and Obese Children During Lifestyle Intervention. J Clin Endocrinol Metab. 2017 Jun 1;102(6):2051-2058.

Dorenbos E, Drummen M, Rijks J, Adam T, Stouthart P, Alfredo Martínez J, Navas-Carretero S, Stratton G, Swindell N, Fogelholm M, Raben A, Westerterp-Plantenga M, Vreugdenhil A. PREVIEW (Prevention of Diabetes Through Lifestyle Intervention and Population Studies in Europe and Around the World) study in children aged 10 to 17 years: Design, methods and baseline results. Diabetes Obes Metab. 2018 May;20(5):1096-1101.

Drummen M, Dorenbos E, Vreugdenhil A, Raben A, Fogelholm M, Westerterp-Plantenga $M$, Adam T. Long-term effects of increased protein intake after weight loss on intrahepatic lipid content and implications for insulin sensitivity: a PREVIEW study. Am J Physiol Endocrinol Metab. 2018 Nov 1;315(5):E885-E891.

Drummen M, Dorenbos E, Vreugdenhil A, Stratton G, Raben A, Westerterp-Plantenga M, Adam T. Associations of Brain Reactivity to Food Cues with Weight Loss, Protein Intake and Dietary Restraint during the PREVIEW Intervention. Nutrients. 2018 Nov 15;10(11). 
Rijks J, Vreugdenhil A, Dorenbos E, Karnebeek K, Joris P, Berendschot T, Mensink R, Plat J. Characteristics of the retinal microvasculature in association with cardiovascular risk markers in children with overweight, obesity and morbid obesity. Scientific Reports 2018 Nov 16;8(1):16952.

Drummen M, Dorenbos E, Vreugdenhil A, Raben A, Westerterp-Plantenga M, Adam T. Insulin resistance, weight, and behavioral variables as determinants of brain reactivity to food cues: a Prevention of Diabetes through Lifestyle Intervention and Population Studies in Europe and around the World - a PREVIEW study. Am J Clin Nutr. 2019 Feb 1;109(2):315-321.

van Dam M, Rijks J, Dorenbos E, Horuz F, van Dael K, Vreugdenhil A. The effect of one year lifestyle intervention on eGFR in children and adolescents with overweight, obesity and morbid obesity. Scientific Reports 2019 Mar 14;9(1):4504.

Drummen M, Heinecke A, Dorenbos E, Vreugdenhil A, Raben A, Westerterp-Plantenga M, Adam T. Reductions in body weight and insulin resistance are not associated with changes in grey matter volume or cortical thickness during the PREVIEW study. J Neurol Sci. 2019 Aug 15;403:106-111.

Karnebeek K, Rijks J, Dorenbos E, Gerver W, Plat J, Vreugdenhil A. Changes in free-living glycemic profiles after 12 months of lifestyle intervention in children with overweight and obesity. Accepted for publication in Nutrients.

Dorenbos E, Drummen M, Adam T, Rijks J, Winkens B, Martinez J, Navas-Carretero S, Stratton G, Swindell N, Stouthart P, Mackintosh K, Ncnarry M, Tremblay A, Fogelholm M, Raben A, Westerterp-Plantenga M, Vreugdenhil A. Effect of a high protein/low Gl diet on insulin resistance in adolescents with overweight/obesity - a PREVIEW Randomized Clinical Trial. Submitted for publication.

Dorenbos E, Drummen M, Adam T, Stratton G, Swindell N, Raben A, Westerterp-Plantenga $M$, Vreugdenhil A. Sleep duration is inversely associated with BMI z-score in children with overweight and obesity, independent of pubertal stage - a PREVIEW Study. Submitted for publication.

Ten Velde G, Plasqui G, Dorenbos E, Winkens B, Vreugdenhil A. Objectively measured physical activity patterns in children with overweight and (morbid) obesity across different weight categories, age groups and gender; baseline data of a multidisciplinary tailored intervention program. Submitted for publication. 
Dorenbos E, Drummen M, Adam TC, Martinez JA, Navas-Carretero S, Stratton G, Swindell $N$, Stouthart P, Fogelholm M, Raben A, Westerterp-Plantenga M, Vreugdenhil A. Role of aminotransferase concentration in insulin resistance and $\mathrm{BMI}$ z-score change in adolescents with overweight/obesity during intervention - a PREVIEW study. Manuscript in progress.

Swindell N, Dorenbos E, Mcnarry M, Mackintosh K, Vreugdenhil A, Stratton G. Cut-off values for Homeostasis Assessment of Insulin Resistance in Children. Manuscript in progress.

Dorenbos E, Timmermans Y, Plat J, Vreugdenhil A. Impact of early life factors on health parameters before and after a lifestyle intervention in children with overweight, obesity and morbid obesity. Submitted for publication. 